\title{
Role of Radical Species in Salicylaldiminato Ni(II) Mediated Polymer Chain Growth: A Case Study for the Migratory Insertion Polymerization of Ethylene in the Presence of MMA.
}

Franz Ölscher ${ }^{\dagger}$, Inigo Göttker-Schnetmann ${ }^{\dagger}$, Vincent Monteil ${ }^{\dagger}$ and Stefan Mecking ${ }^{\dagger, *}$.

${ }^{\dagger}$ Chair of Chemical Materials Science, Department of Chemistry, University of Konstanz, D-78464 Konstanz, Germany

tUniversité de Lyon, Univ. Lyon 1, CPE Lyon, CNRS UMR 5265 Laboratoire de Chimie Catalyse Polymères et Procédés (C2P2), LCPP team Bat 308F, 43 Bd du 11 novembre 1918, F-69616 Villeurbanne, France

Supporting Information 


\section{Contents}

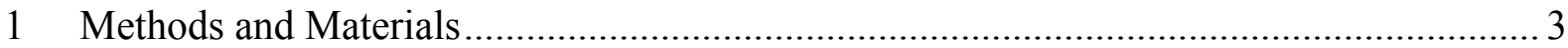

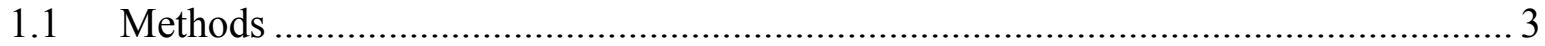

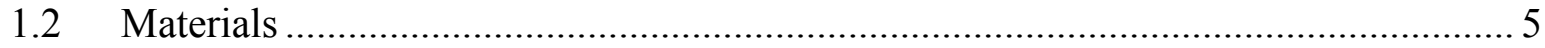

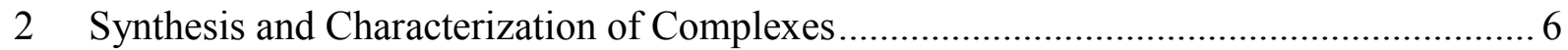

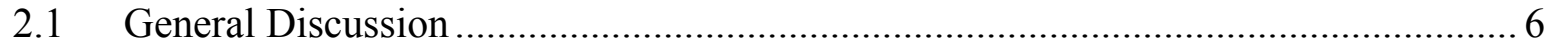

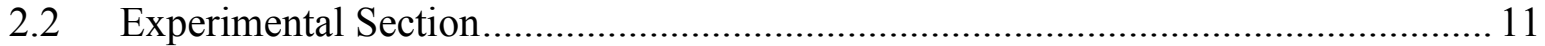

3 Reactivity of Different Ni Catalyst Precursors toward MMA .......................................26

3.1 Polymerization of MMA initiated with $\left[\left(\mathrm{N}^{\wedge} \mathrm{O}\right) \mathrm{Ni}(\mathrm{II}) \mathrm{R}\left(\mathrm{PPh}_{3}\right)\right]$ complexes.............. 26

3.2 Microstructure and end-group analysis of PMMA homopolymers......................... 28

4 Reactivity of MMA toward Insertion Chain Growth Species....................................... 32

4.1 Studies on the migratory insertion initiation process using labeled $\left[\mathrm{Ni}^{-}{ }^{13} \mathrm{CH}_{3}\right]$ and

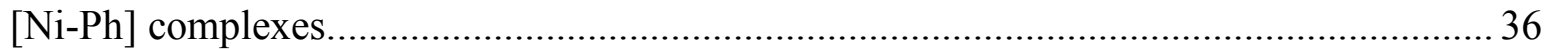

4.2 DOSY, SEC and extraction experiments of polymers obtained from simultaneous polymerization of MMA and ethylene

5 Stoichiometric Reactions of $\left(\mathrm{N}^{\wedge} \mathrm{O}\right) \mathrm{Ni}(\mathrm{II})$-catalyst Precursors and Intermediate [Ni]Species toward MMA.

5.1 General procedure for NMR experiments.

5.2 Additional discussion of the observed reactivity of $\left(\mathrm{N}^{\wedge} \mathrm{O}\right) \mathrm{Ni}$ species by spectroscopic methods

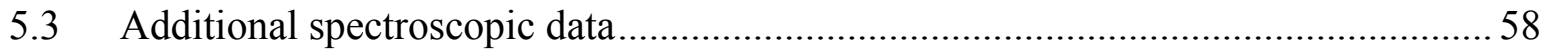

6 Sources of Organic Free Radicals in $\left(\mathrm{N}^{\wedge} \mathrm{O}\right) \mathrm{Ni}(\mathrm{II}) \mathrm{R}(\mathrm{L})$ Mediated Polymerizations .......... 71

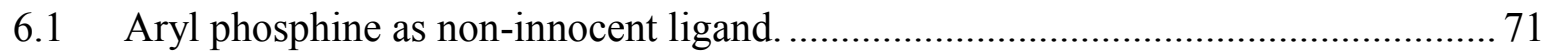

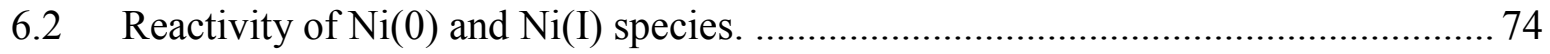

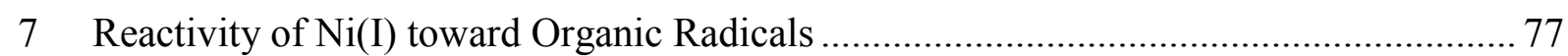

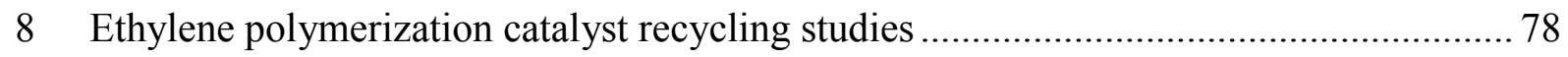

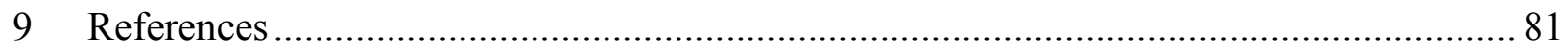




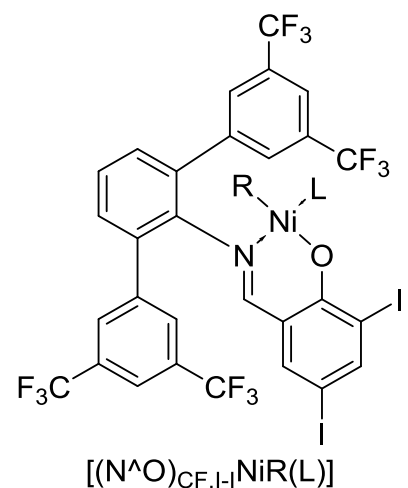
$\left[\left(\mathrm{N}^{\wedge} \mathrm{O}\right)_{\mathrm{CF}, \mathrm{I-I}} \mathrm{NiR}(\mathrm{L})\right]$ $1_{\mathrm{L}}^{\mathrm{R}}$

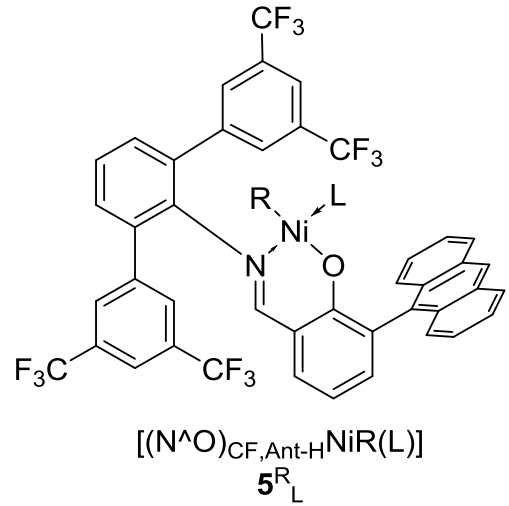<smiles></smiles>

$\left[\left(\mathrm{N}^{\wedge} \mathrm{O}\right)_{i \mathrm{Pr}, \mathrm{I-I}} \mathrm{NiR}(\mathrm{L})\right]$ $2^{\mathrm{R}}$<smiles></smiles>

$\left[\left(\mathrm{N}^{\wedge} \mathrm{O}\right)_{i \mathrm{Pr}, t \mathrm{Bu}-\mathrm{H}} \mathrm{NiR}(\mathrm{L})\right]$ $3^{R}$

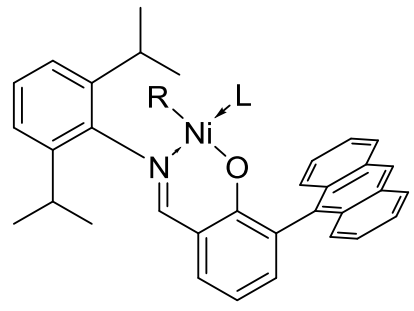

$\left[\left(\mathrm{N}^{\wedge} \mathrm{O}\right)_{\text {iPr,Ant-H}} \mathrm{NiR}(\mathrm{L})\right]$ $4^{R}$
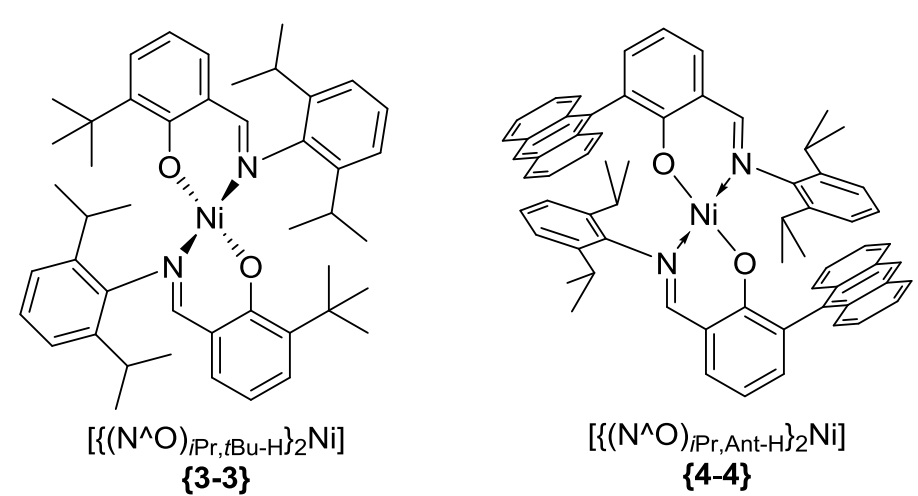

$\left[\left\{\left(\mathrm{N}^{\wedge} \mathrm{O}\right)_{i \mathrm{Pr}, \mathrm{Ant}-\mathrm{H}}\right\}_{2} \mathrm{Ni}\right]$

\{4-4\}

Chart S 1. Chemical structures, general notation and abbreviations for the complexes used in this publication.

\section{Methods and Materials}

\subsection{Methods}

NMR spectra were recorded on a Varian Unity Inova 400 equipped with a 4NUC/switchable mode - $5 \mathrm{~mm}$ "direct detection" probe, a Bruker Avance III 400 equipped with a BBFO plus probe, a Bruker Avance III 600 equipped with a QXI-H/C/N/P quadruple resonance probe or a Bruker Avance III 600 equipped with a TCI-H/C/N triple resonance cryoprobe. ${ }^{1} \mathrm{H}$ NMR spectra were referenced to residual protiated solvent signals. ${ }^{13} \mathrm{C}$ NMR spectra were referenced to the solvent signals, ${ }^{31} \mathrm{P}$ NMR spectra to external $85 \% \mathrm{H}_{3} \mathrm{PO}_{4}$ and ${ }^{19} \mathrm{~F}$ NMR spectra to external $\mathrm{BF}_{3} \cdot \mathrm{OEt}_{2}$. NMR spectra are reported as follows: chemical shift $(\delta \mathrm{ppm})$, multiplicity, coupling constant $(\mathrm{Hz})$ and integration. Multiplicities are given as follows (or combinations thereof): $\mathrm{s}$ : singlet, d: doublet, t: triplet, q: quartet, quint: quintet, sept: septet, m: multiplet, v: virtual, br.: broad. The identity of metal complexes and detailed NMR assignments were established by 2D NMR experiments $\left({ }^{1} \mathrm{H}-{ }^{1} \mathrm{H}\right.$ COSY, ${ }^{1} \mathrm{H}-{ }^{1} \mathrm{H}$ ROESY/NOESY, ${ }^{1} \mathrm{H}-{ }^{13} \mathrm{C} \mathrm{HSQC},{ }^{1} \mathrm{H}-{ }^{13} \mathrm{C} \mathrm{HMBC}$ and $\left.{ }^{1} \mathrm{H}^{31} \mathrm{P} \mathrm{HMBC}\right)$ in addition to $1 \mathrm{D}$ NMR experiments. Acquired data were processed and analyzed using MestReNova software. DOSY data were processed using the TopSpin (Bruker) software package. 
X-band continuous wave (cw) EPR spectra $(9.4 \mathrm{GHz})$ were recorded on a MiniScope MS400 (Magnettech $\mathrm{GmbH}$, Germany) equipped with a liquid nitrogen cooling system for the cavity. The magnetic field was modulated by $100 \mathrm{kHz}$. Samples of air/moisture sensitive compounds were dissolved, transferred to EPR tubes and sealed with a rubber septa fixed with Teflon tape in a $\mathrm{N}_{2}$ filled glovebox. To determine the hyperfine structure spectra were simulated using the EasySpin toolbox ${ }^{1}$ for MatLab.

Single crystal X-ray diffraction analysis was performed at $100 \mathrm{~K}$ on a STOE IPDS-II diffractometer equipped with a graphite-monochromated radiation source $(\lambda=0.71073 \AA)$ and an image plate detection system. Crystals were mounted on a fine glass fiber with silicon grease. The selection, integration and averaging procedure of the measured reflex intensities, the determination of the unit cell dimensions and a least-squares fit of the $2 \theta$ values as well as data reduction, LP-correction and space group determination were performed using the X-Area software package delivered with the diffractometer. ${ }^{2}$ A semi empirical absorption correction was performed. The structures were solved by Patterson or Direct methods (SHELXS 97 or SHELXS-2013 ${ }^{3}$ ), completed with difference Fourier synthesis, and refined with full-matrix least-square using SHELXL97 or SELXL-2013 ${ }^{3}$ minimizing $\mathrm{w}\left(F_{0}{ }^{2}-F_{c}{ }^{2}\right)^{2}$. Weighted R factor $\left(w R_{2}\right)$ and the goodness of fit (GooF) are based on $F^{2}$. All non-hydrogen atoms were refined with anisotropic displacement parameters. All hydrogen atoms were treated in a riding model unless otherwise noted. Graphical output (ORTEP plots) were created using ORTEP-3 V. 2.02 for Windows. ${ }^{4}$

GC-MS spectra were recorded on a HP-6890 GC-system with a HP5973 MSD.

Matrix-assisted laser desorption/ionization time of flight mass spectroscopy (MALDI-TOF MS) were recorded using a Microflex mass spectrometer (Bruker Daltonics), equipped with a 335 $\mathrm{nm}$ nitrogen laser and operated in the linear mode. As a matrix, a saturated solution of dithranol in THF with $\mathrm{NaI}$ as doping salt, with addition of $0.1 \%$ trifluoracetic acid, was used. For sample preparation, $1 \mu \mathrm{L}$ of the matrix solution was spotted on the MALDI target, dried, and $0.7 \mu \mathrm{L}$ of the sample solution ( $10 \mu \mathrm{g} / \mu \mathrm{L}$ in chloroform) was added to the MALDI spot and dried again.

Differential scanning calorimetry (DSC) of polymers were measured on a Netzsch DSC 204 F1 with a heating/cooling rate of $10 \mathrm{~K} \mathrm{~min}^{-1}$. Data reported for melting and glass transitions are reported from the second heating cycle unless noted otherwise. Crystallinities were calculated assuming $\Delta H_{\mathrm{m}}=293 \mathrm{~J} \mathrm{~g}^{-1}$ for $100 \%$ crystallinity of PE. ${ }^{5}$

Size exclusion chromatography (SEC) for molecular weight determination was carried out on a Polymer Laboratories PL-GPC 50 with two PLgel $5 \mu \mathrm{m}$ MIXED-C columns in THF at $40{ }^{\circ} \mathrm{C}$ against polystyrene (PS) standards with refractive index detection or on a Polymer Laboratories 
220 instrument equipped with Olexis columns with differential refractive index, viscosity and light scattering $\left(15^{\circ}\right.$ and $\left.90^{\circ}\right)$ detectors in 1,2,4-trichlorobenzene (TCB) at $160^{\circ} \mathrm{C}$ at a flow rate of $1 \mathrm{~mL} \cdot \mathrm{min}^{-1}$. Data reported were determined in linear mode vs. linear PE standards.

Elemental analysis was carried out on an Elementar vario MICRO cube instrument at the Department of Chemistry at the University of Konstanz.

\subsection{Materials}

All manipulations of nickel complexes were carried out under an inert atmosphere $\left(\mathrm{N}_{2}\right)$ using standard glovebox or Schlenk techniques. All glassware was dried under vacuum prior to use. Diethylether, THF and benzene were distilled from blue sodium/benzophenone and toluene from sodium. $\mathrm{MeOH}$ was distilled from activated magnesium. Pentane was distilled from calcium hydride. All solvents were distilled under inert atmosphere $\left(\mathrm{N}_{2}\right)$.

Deuterated solvents were purchased from Euriso-Top. 1,1,2,2-tetrachloroethane- $d_{2}$ (TCE- $d_{2}$ ) was stored over molecular sieves $(4 \AA)$. THF- $d_{8}$ was dried over $\mathrm{Na} / \mathrm{K}$ alloy and distilled prior to use. DMSO- $d_{6}, \mathrm{C}_{6} \mathrm{D}_{6}$ and toluene- $d_{8}$ was dried over $\mathrm{CaH}_{2}$ and vacuum transferred prior to use.

Methyl methacrylate containing $<30$ ppm hydrochinon-monomethylether (MEHQ) as stabilizer was purchased from Sigma Aldrich and dried over $\mathrm{CaH}_{2}$. Prior to use MMA was carefully vacuum transferred at room temperature to remove impurities and radical inhibitor and degassed by three pump-freeze-thaw cycles. Ethylene (3.5 grade) was supplied by Praxair.

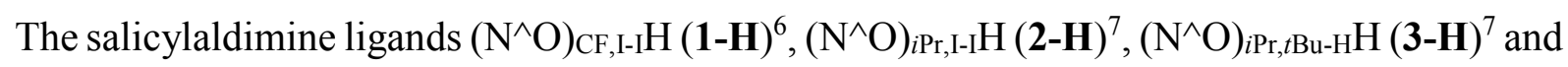
$\left(\mathrm{N}^{\wedge} \mathrm{O}\right)_{i \mathrm{Pr}, \mathrm{Ant}-\mathrm{H} H}(\mathbf{4}-\mathrm{H})^{7}$ were synthesized following literature procedures. The deprotonated ligands 1-Na, 2-Na, 3-Na and 4-Na were obtained by reaction with $\mathrm{NaH}$ in THF according to literature procedures. ${ }^{7}$

Synthesis of phenylazotriphenylmethane, ${ }^{8}$ transchloro(phenyl)bis(triphenylphosphine)nickel(II) $\quad\left(\left[\left(\mathrm{PPh}_{3}\right)_{2} \mathrm{Ni}(\mathrm{Cl}) \mathrm{Ph}\right]\right),{ }^{9} \quad \mathbf{1}^{\mathrm{Cl}}{ }_{\mathrm{PMe}}$

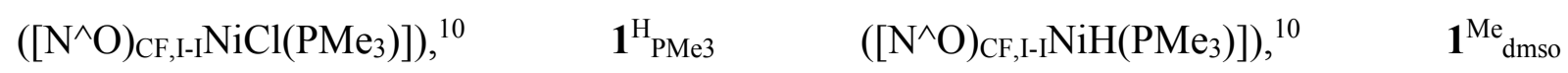

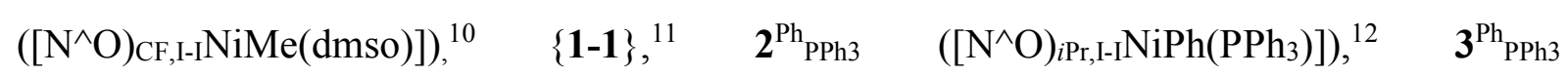

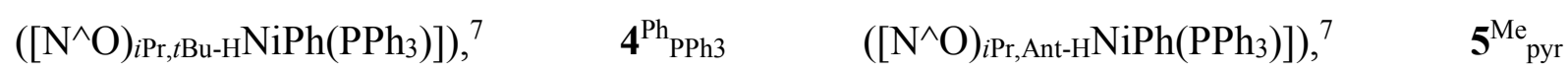

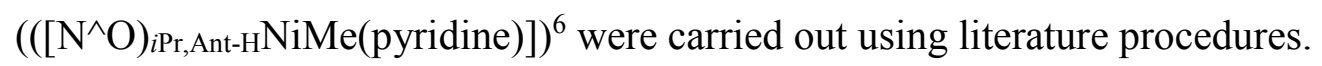




\section{Synthesis and Characterization of Complexes}

\subsection{General Discussion}

Synthesis of metal-centered radical Ni(I) complexes. Note that in the past years several $\mathrm{Ni}(\mathrm{I})$ complexes have been synthezized ${ }^{13}$ and drawn much attention because it has been shown that many cross coupling reactions proceed in a non-classical $\mathrm{Ni}\left(\mathrm{I} / \mathrm{Ni}\right.$ (III) pathway. ${ }^{14}$ In the present study it is of significance that any homolytic cleavage of the $\mathrm{M}-\mathrm{C}$ bond in the polymerization active species - e.g. in bimolecular reductive elimination or with the release of an organic free radical - should yield a $\mathrm{Ni}(\mathrm{I})$ species. Therefore, we developed an independant synthethic approach to the anticipated $\mathrm{Ni}(\mathrm{I})$ salicylaldiminato complex.

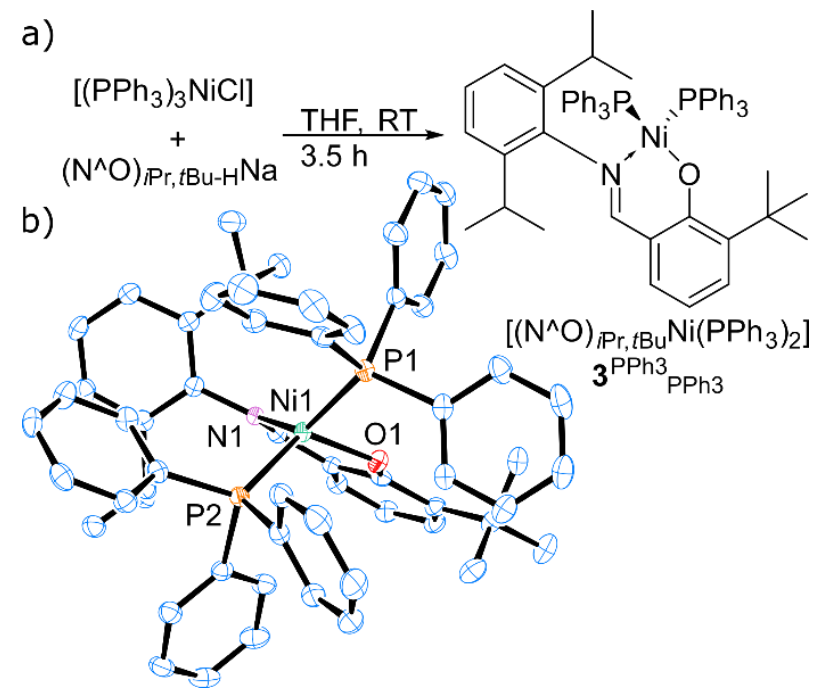

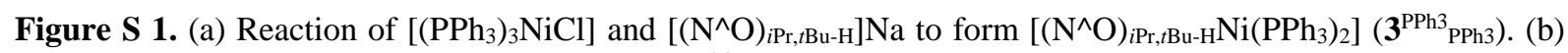
ORTEP ( $50 \%$ probability ellipsoids) plot of $3^{\mathrm{PPh} 3}{ }_{\mathrm{PPh} 3}$ determined by X-ray diffraction. Hydrogen atoms are omitted for clarity. One possible pentane molecule in a solvent accesible void was removed by the PLATON SQUEEZE ${ }^{15}$ routine.

The $\mathrm{Ni}(\mathrm{I})$ precursor $\left[\left(\mathrm{PPh}_{3}\right)_{3} \mathrm{NiCl}\right]$ was synthesized by comproportionation of $\left[\mathrm{Ni}\left(\mathrm{PPh}_{3}\right)_{4}\right]$ and [ $\left.\left(\mathrm{PPh}_{3}\right)_{2} \mathrm{NiCl}_{2}\right]$ in $\mathrm{Et}_{2} \mathrm{O}$ according to Heimbach. ${ }^{16}$ Subsequent reaction with 1 equiv. of

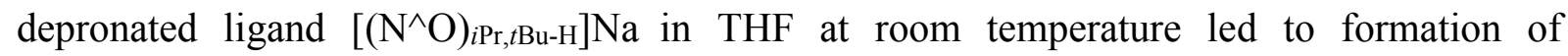

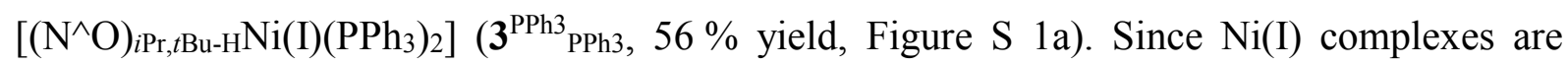
generally not thermodynamically stable compounds, it is surpising that $3^{\mathrm{PPh} 3}{ }_{\mathrm{PPh} 3}$ was stable over hours at elevated temperatures (Figure S 7). Traces of water and/or air decreased the stability dramatically and the final main decomposition products were the bis-hydroxy bridged $\mathrm{Ni}(\mathrm{II})$

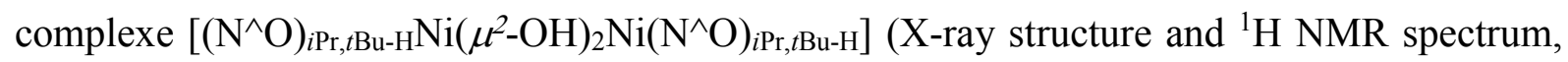
Figure S 14 and Figure S 15, respectively) and phosphine oxide.

Crystals of $3^{\mathrm{PPh} 3}{ }_{\mathrm{PPh} 3}$ suitable for X-ray crystallography were grown from a saturated pentane solution at $-24{ }^{\circ} \mathrm{C} .3^{\mathrm{PPh} 3}{ }_{\mathrm{PPh} 3}$ crystallizes in a triclinic crystal system and adopts a distorted tetragonal coordination geometry (Figure $\mathrm{S} \quad 1 \mathrm{~b}$ ). The dihedral angle 
$\varepsilon(\angle\{\mathrm{P} 1-\mathrm{Ni1}-\mathrm{P} 2\}-\{\mathrm{N} 1-\mathrm{Ni1}-\mathrm{O} 1\})$ is strongly distorted $\left(65.83^{\circ}\right)$ from an optimal tetrahedral $\left(\varepsilon=90^{\circ}\right)$, whereas $\varepsilon\left(\angle\{\mathrm{P} 1-\mathrm{Ni1}-\mathrm{N} 1\}-\{\mathrm{P} 2-\mathrm{Ni1}-\mathrm{O} 1\}=90.39^{\circ}\right.$ shows almost a perfect angle for tetrahedral coordination geometry. ${ }^{17}$ As a result of the distorted tetragonal coordination geometry $\mathrm{P} 1$ is located closer to the bulky $t \mathrm{Bu}$ substituent in the 3 position of the salicylaldimine, which results in a slightly longer Ni1-P1 bond (2.2611(6) $\AA$ ) than for Ni1-P2 (2.2580(6) A). The Ni-P bond distances are significantly longer compared to the corresponding

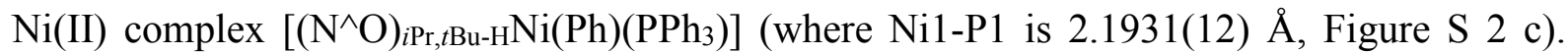
Assuming a similar electronic density resulting from the $\left(\mathrm{N}^{\wedge} \mathrm{O}\right)$ ligand, one would expect shorter Ni-P distances in the reduced form, because this should lead to a stronger $\pi$-back bonding, ${ }^{18}$ however, this is not observed here. The steric demand of two $\mathrm{PPh}_{3}$ ligands seems to predominate over electronic effects, favoring a tetrahedral geometry. The $\mathrm{N}^{\wedge} \mathrm{O}$ coordination in $3^{\mathrm{PPh} 3}{ }_{\mathrm{PPh} 3}$ is weaker in comparison to $3^{\mathrm{Ph}}{ }_{\mathrm{PPh} 3}$, as concluded from Ni1-N1 (2.0170(15) $\AA$ ) and N1O1 (1.9792(13) $\AA$ ) bonds, which are longer than Ni1-N1 and Ni1-O1 in

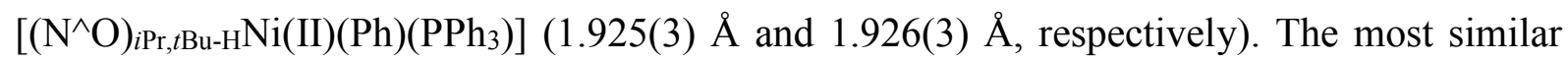
complexes for which $\mathrm{X}$-ray diffraction data is available are the trigonal planar anilido-imine triphenylphosphine $\mathrm{Ni}(\mathrm{I})$ complexes synthesized by Wang et al. in $2006 .{ }^{19} \mathrm{Ni}-\mathrm{N}$ herein are $1.970(4) \AA$ and 1.933(2) $\AA$, both being shorter than in the four coordinate $\left(\mathrm{N}^{\wedge} \mathrm{O}\right) \mathrm{Ni}(\mathrm{I})$ complex $3^{\mathrm{PPh} 3}{ }_{\mathrm{PPh} 3}(2.0170(15) \AA)$, which can be accounted for by steric effects resulting from the additional $\mathrm{PPh}_{3}$ in the ligand sphere. The same trend is observed for both Ni-P bonds in $3^{\mathrm{PPh} 3} \mathrm{PPh} 3$, which are 2.2611(6) $\AA$ for Ni1-P1 and for 2.2580(6) $\AA$ Ni1-P2, in comparison to 2.2096(9) $\AA$ for $\mathrm{Ni}-\mathrm{P}$ in $\left[\left(\mathrm{N}^{\wedge} \mathrm{N}\right) \mathrm{Ni}(\mathrm{I})\left(\mathrm{PPh}_{3}\right)\right]$.

The continous wave EPR spectrum in toluene at $5{ }^{\circ} \mathrm{C}$ (Figure S 5) shows an isotropic resonance with a $g$ value of 2.20 with no hyperfine structure (HFS) resolved. In glassy toluene solution $\left(-170{ }^{\circ} \mathrm{C}\right.$, Figure S 6$)$ a rhombic $g$ tensor $\left(2.07,2.12,2.34 ; g_{i s o}=2.20\right)$ with values typical for metal centered radical $\mathrm{S}=1 / 2[\mathrm{Ni}(\mathrm{I})]$ complexes was observed. ${ }^{20}$ For all three $g$ values a large hyperfine splitting of two ${ }^{31} \mathrm{P}$ nuclei $\left(A(\mathrm{P} 1)_{\text {iso }}=164 \mathrm{MHz}, A(\mathrm{P} 2)_{\text {iso }}=151 \mathrm{MHz}\right)$ was resolved (values derived from simulation of the EPR spectrum).

$\left(N^{\wedge} O\right) N i(I I)(R)(L)$ catalyst precursors. [Ni-Ph] complexes are the most widely used $\kappa^{2}-\mathrm{N}^{\wedge} \mathrm{O}$ $\mathrm{Ni}$ (II) catalyst precursors, [Ni-Me] complexes resemble catalysts precursors which more closely represent the growing Ni-polymeryl species. $[\mathrm{Ni}-\mathrm{H}]$ represents the highly reactive intermediates formed upon chain transfer by $\beta$-H elimination. 
Scheme S 1. Synthesis of $[\mathrm{Ni}-\mathrm{H}]$ complexes by reaction of $\left[\left(\mathrm{N}^{\wedge} \mathrm{O}\right) \mathrm{NiCl}(\mathrm{L})\right]$ with $\mathrm{NaHB}(\mathrm{OMe})_{3}$.

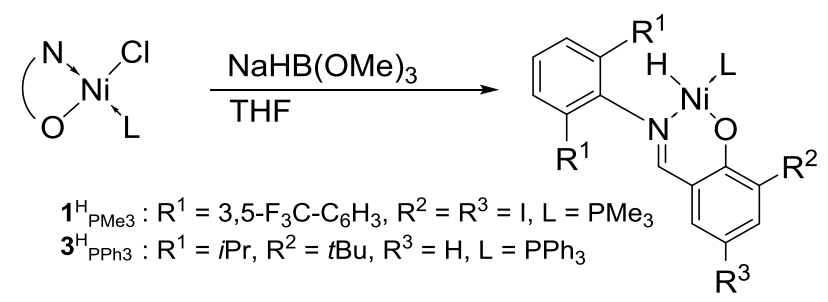

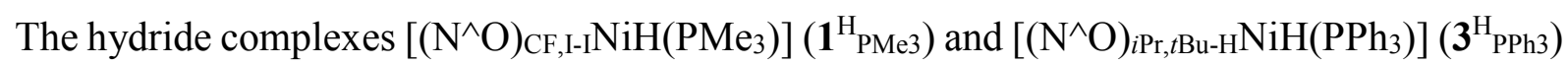
were obtained by reaction of the corresponding $\left[\left(\mathrm{N}^{\wedge} \mathrm{O}\right) \mathrm{NiCl}\left(\mathrm{PR}_{3}\right)\right]$ precursor complex with $\mathrm{NaHB}(\mathrm{OMe})_{3}$ in THF (Scheme S 1). ${ }^{10}$ The new in-situ formed hydride complex $3^{\mathrm{H}_{\mathrm{PPh}}}$ shows a characteristic ${ }^{1} \mathrm{H}$ NMR (THF- $d_{8}$, RT) doublet at -27.6 ppm with a ${ }^{2} J_{\mathrm{H}-\mathrm{P}}$ coupling of $140.2 \mathrm{~Hz}$ to the $c$ is phosphorus $\left(\delta\left({ }^{31} \mathrm{P}\right)=26.1 \mathrm{ppm}\right)$. The successful formation at room temperature and the high thermal stability of $3^{\mathrm{H}}$ PPh is somewhat surprising when compared to the reactivity of $\mathbf{1}_{\text {PMe3 }}^{\mathrm{H}} \mathbf{3}_{\mathrm{PPh} 3}^{\mathrm{H}}$ is accessible in-situ at RT, even with less stabilizing phosphine $\mathrm{PPh}_{3}$ as a labile ligand.

Scheme S 2. Synthesis of [Ni-Me] complexes starting from [(tmeda) $\left.\mathrm{NiMe}_{2}\right]$ and protonated $\mathrm{N}^{\wedge} \mathrm{O}$-ligand.

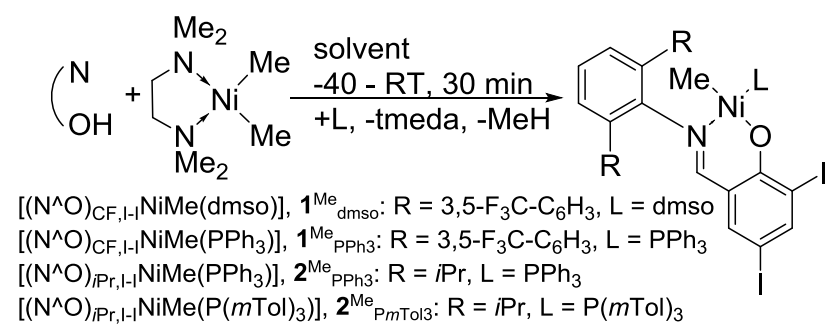

The [Ni-Me] complexes $\mathbf{1}^{\mathrm{Me}}{ }_{\text {dmso }}{ }^{10}, \mathbf{1}^{\mathrm{Me}}{ }_{\mathrm{PPh} 3}{ }^{21}, \mathbf{2}^{\mathrm{Me}}{ }_{\mathrm{PPh}}{ }^{21}$ and the novel complex $\mathbf{2}^{\mathrm{Me}}{ }_{\mathrm{P} m \mathrm{Tol} 3}$ were synthesized by reaction of the corresponding protonated ligand $\left(\mathrm{N}^{\wedge} \mathrm{O}\right) \mathrm{H}$ with 1.2 equiv. [(tmeda)NiMe $\mathrm{Nin}_{2}$ in the presence of the respective labile ligand (Scheme $\mathrm{S}_{2}$ ). The ${ }^{13} \mathrm{C}$ labeled complexes $1^{13 \mathrm{CH} 3}{ }_{\mathrm{PPh} 3}$ and $2^{13 \mathrm{CH} 3}{ }_{\mathrm{PPh} 3}$ were synthesized by procedures published elsewhere. ${ }^{21}$ The synthesis of $\mathbf{2}^{\mathrm{Me}}{ }_{\mathrm{P} m \mathrm{Tol} 3}$ was performed similar to reported procedures, with a modified work up. The reaction mixture was washed with cold $\mathrm{MeOH}$, extracted with pentane and precipitated from solution by cooling to $-24^{\circ} \mathrm{C} .2^{\mathrm{Me}}{ }_{\mathrm{P} m \mathrm{Tol}}$ was obtained in satisfactory yield $(73 \%)$ as an orange powder. 
Scheme $\mathrm{S}$ 3. Synthesis of $[\mathrm{Ni}-\mathrm{Ph}]$ complexes by reaction of deprotonated salicylaldimine with trans$\left[\left(\mathrm{PPh}_{3}\right)_{2} \mathrm{NiCl}(\mathrm{Ph})\right]$ according to the synthetic route of Wang et al.

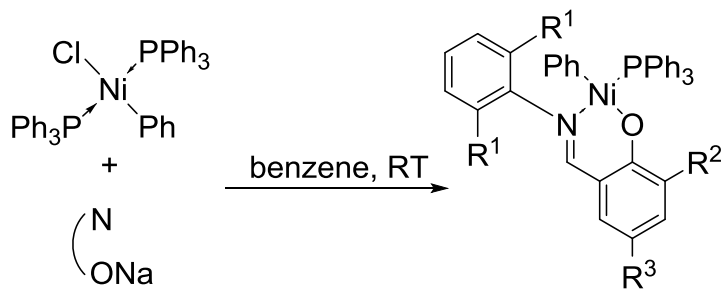

$\left[\left(\mathrm{N}^{\wedge} \mathrm{O}\right)_{\mathrm{CF}, \mathrm{II}-1} \mathrm{NiPh}\left(\mathrm{PPh}_{3}\right)\right], \mathbf{1}^{\mathrm{Ph}} \mathrm{PPh}_{3}: \mathrm{R}^{1}=3,5-\mathrm{F}_{3} \mathrm{C}-\mathrm{C}_{6} \mathrm{H}_{3}, \mathrm{R}^{2}=\mathrm{R}^{3}=\mathrm{I}$

$\left[\left(\mathrm{N}^{\wedge} \mathrm{O}\right)_{\mathrm{PP}, \mathrm{I},-1} \mathrm{NiPh}\left(\mathrm{PPh}_{3}\right)\right], \mathbf{2}^{\mathrm{Ph}}{ }_{\mathrm{PPh} 3}: \mathrm{R}^{1}=\mathrm{iPr}, \mathrm{R}^{2}=\mathrm{R}^{3}=1$

$\left[\left(\mathrm{N}^{\wedge} \mathrm{O}\right)_{\mathrm{PP}, t \mathrm{Bu}-\mathrm{H}} \mathrm{NiPh}\left(\mathrm{PPh}_{3}\right)\right], 3^{\mathrm{Ph}}{ }_{\mathrm{PPh} 3}: \mathrm{R}^{1}=\operatorname{Pr}, \mathrm{R}^{2}=t \mathrm{Bu}, \mathrm{R}^{3}=\mathrm{H}$

$\left[\left(\mathrm{N}^{\wedge} \mathrm{O}\right)_{\mathrm{PPr}, \mathrm{Ant}-\mathrm{H}} \mathrm{NiPh}\left(\mathrm{PPh}_{3}\right)\right], 4_{\mathrm{PPh} 3}^{\mathrm{Ph}}: \mathrm{R}^{1}=i \mathrm{Pr}, \mathrm{R}^{2}=\mathrm{Ant}, \mathrm{R}^{3}=\mathrm{H}$

[Ni-Ph] complexes $2^{\mathrm{Ph}}{ }_{\mathrm{PPh} 3},{ }^{12} 3^{\mathrm{Ph}}{ }_{\mathrm{PPh} 3}{ }^{7}$ and $4^{\mathrm{Ph}}{ }_{\mathrm{PPh} 3}{ }^{7}$ were obtained by reaction of the corresponding $\left(\mathrm{N}^{\wedge} \mathrm{O}\right) \mathrm{Na}$ salt with trans- $\left[\left(\mathrm{PPh}_{3}\right)_{2} \mathrm{NiCl}(\mathrm{Ph})\right]^{9}$ in benzene according to literature procedures (Scheme $\left.\mathrm{S}_{3}\right) . \mathbf{1}^{\mathrm{Ph}}{ }_{\mathrm{PPh}}$ has not been reported previously, and was obtained by a modification of the aforementioned literature known protocols. The crude reaction mixture was extracted with cold $\mathrm{MeOH}$, reduced to dryness, washed with small amounts of pentane and $\mathbf{1}^{\mathrm{Ph}}{ }_{\mathrm{PPh} 3}$ was obtained in satisfactory yields (72\%) as an orange powder.

Complexes $\mathbf{1}^{\mathrm{Ph}}{ }_{\mathrm{PPh} 3}$ (CCDC 1432837), $\mathbf{2}^{\mathrm{Ph}}{ }_{\mathrm{PPh} 3}$ (CCDC 1432838) and $\mathbf{3}^{\mathrm{Ph}}{ }_{\mathrm{PPh} 3}$ (CCDC 1432841) were analyzed by X-ray diffraction (selected bond distances and angles see Table S 1, ORTEP plots Figure S 2). Crystals suitable for X-ray diffraction were obtained from saturated solutions of $\mathrm{Et}_{2} \mathrm{O}$ /pentane for $\mathbf{1}^{\mathrm{Ph}}{ }_{\mathrm{PPh} 3}$ and pentane for $\mathbf{2}^{\mathrm{Ph}}{ }_{\mathrm{PPh}}, \mathbf{3}^{\mathrm{Ph}}{ }_{\mathrm{PPh} 3}$ at $-26^{\circ} \mathrm{C}$. $\mathbf{2}^{\mathrm{Ph}}{ }_{\mathrm{PPh} 3}$ presumably cocrystallized with two disordered pentane molecules per unit cell, which could not be resolved and were removed by the PLATON ${ }^{15}$ SQUEEZE routine.
a) $\left[\left(\mathrm{N}^{\wedge} \mathrm{O}\right)_{\mathrm{CF},-1-1} \mathrm{NiPh}\left(\mathrm{PPh}_{3}\right)\right], 1^{\mathrm{Ph}} \mathrm{PPh}_{3}$
b) $\left[\left(\mathrm{N}^{\wedge} \mathrm{O}\right)_{\mathrm{PPr},-1-1} \mathrm{NiPh}\left(\mathrm{PPh}_{3}\right)\right], \mathbf{2}^{\mathrm{Ph}}{ }_{\mathrm{PPh} 3}$
c) $\left[\left(\mathrm{N}^{\wedge} \mathrm{O}\right)_{\mathrm{iPr}, \mathrm{BBu}-\mathrm{H}} \mathrm{NiPh}\left(\mathrm{PPh}_{3}\right)\right], 3^{\mathrm{Ph}}{ }_{\mathrm{PPh} 3}$
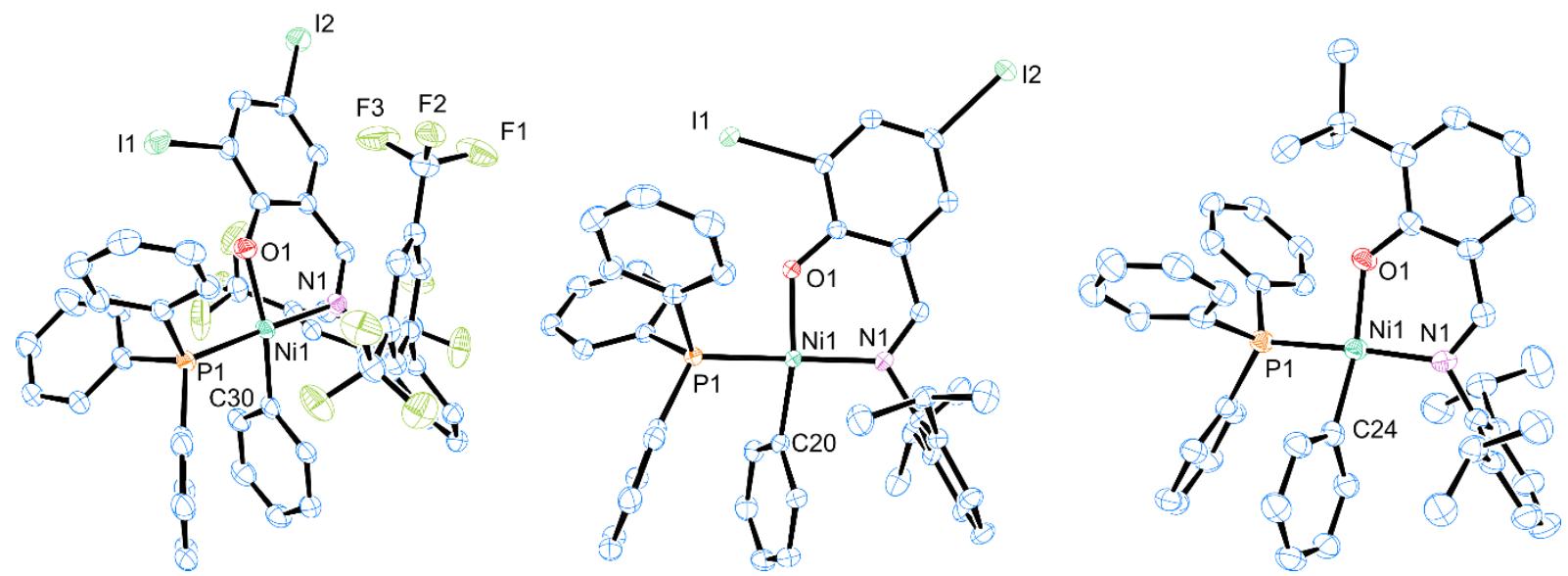

Figure S 2. ORTEP (50\% probability ellipsoids) plots of $\mathbf{1}^{\mathrm{Ph}}{ }_{\mathrm{PPh} 3}(\mathrm{a}), 2^{\mathrm{Ph}}{ }_{\mathrm{PPh} 3}$ (b) and $3^{\mathrm{Ph}}{ }_{\mathrm{PPh} 3}$ (c) determined by X-ray diffraction. Hydrogen atoms are omitted for clarity. The structure of $2^{\mathrm{Ph}}{ }_{\mathrm{PPh} 3}$ contains presumably two disordered pentane molecules and were removed by the PLATON SQUEEZE ${ }^{15}$ routine.

All three complexes crystallize in a square planar coordination geometry with the phenyl group located trans to the oxygen atom of the salicylaldimine. The Nil-P1 bond lengths are similar in 
complexes $\mathbf{1}^{\mathrm{Ph}}{ }_{\mathrm{PPh} 3}$ and $\mathbf{2}^{\mathrm{Ph}}{ }_{\mathrm{PPh} 3}\left(2.1747(7) \AA\right.$ and $2.1766(6) \AA$, respectively), whereas in $3^{\mathrm{Ph}}{ }_{\mathrm{PPh} 3}$ Ni1-P1 is significantly longer (2.1931(12) $\AA$ ).

Table S 1. Selected crystallographic bond distances and bond angles for the $[\mathrm{Ni}-\mathrm{Ph}]$ complexes $\mathbf{1}^{\mathrm{Ph}}{ }_{\mathrm{PPh} 3}, \mathbf{2}^{\mathrm{Ph}}{ }_{\mathrm{PPh} 3}$ and $3^{\mathrm{Ph}}{ }_{\mathrm{PPh} 3}$.

\begin{tabular}{|c|c|c|c|}
\hline Bond /Angle & $\mathbf{1}^{\mathrm{Ph}_{\mathrm{PPh} 3}}$ & $2^{\mathrm{Ph}_{\mathrm{PPh}} 3}$ & $3^{\mathrm{Ph}_{\mathrm{PPh}}}$ \\
\hline Ni1-P1 (̊̊) & $2.1747(7)$ & $2.1766(6)$ & $2.1931(12)$ \\
\hline Ni1-C (Å̀) & $1.908(3)$ & $1.905(2)$ & $1.916(4)$ \\
\hline Ni1-O1 (Å) & $1.906(2)$ & $1.903(1)$ & $1.926(3)$ \\
\hline Ni1-N1 (̊̊) & $1.946(2)$ & $1.938(2)$ & $1.925(3)$ \\
\hline P1-Ni1-N1 ( $\left.{ }^{\circ}\right)$ & $177.00(7)$ & $166.78(5)$ & $169.7(1)$ \\
\hline O1-Ni1-C $\left(^{\circ}\right)$ & $167.8(1)$ & $162.50(7)$ & $164.4(1)$ \\
\hline P1-Ni1-O1 ( $\left.{ }^{\circ}\right)$ & $90.04(6)$ & $87.72(5)$ & $90.22(8)$ \\
\hline C-Ni-N1 $\left(^{\circ}\right)$ & $94.1(1)$ & $96.64(7)$ & $94.3(1)$ \\
\hline
\end{tabular}

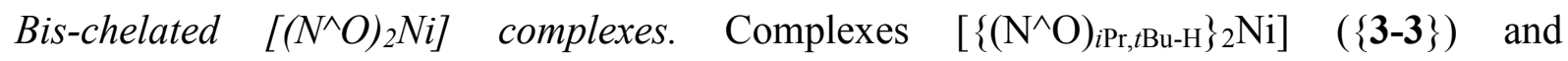

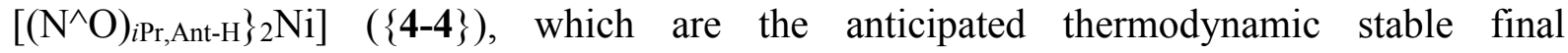
decomposition products, were synthesized by reaction of the corresponding $\left(\mathrm{N}^{\wedge} \mathrm{O}\right) \mathrm{Na}$-salt with $\left[(\mathrm{dmso})_{3} \mathrm{NiCl}_{2}\right]$ in good yields $\left(68 \%\right.$ and $93 \%$, respectively, Scheme $\left.\mathrm{S}_{4}\right)$ and characterized by NMR, elemental analysis and X-ray diffraction.

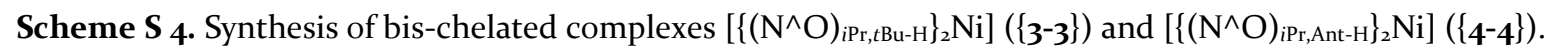

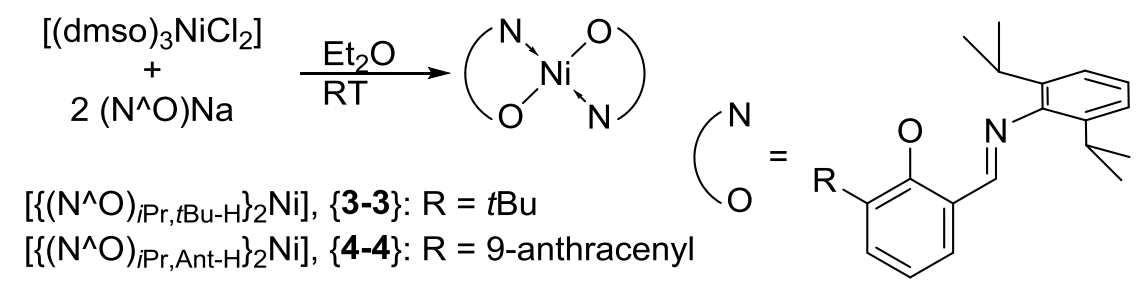

Different synthetic approaches for both complexes were published previously. ${ }^{22}$ Complex $\{3-3\}$ (CCDC 1432839) was characterized by X-ray diffraction and shows that the steric bulk of the $t \mathrm{Bu}$ group in the 3-position of the salicylaldimine forces $\{3-3\}$ into a tetrahedral coordination geometry, leading to a paramagnetic $\mathrm{S}=1$ spin state complex. In accordance, chemical shifts ranging from -22 to $282 \mathrm{ppm}$ were observed in the ${ }^{1} \mathrm{H}$ NMR spectrum (toluene- $d_{8}$, RT, 400 $\mathrm{MHz}$ ). $\{3-3\}$ reacts with traces of water forming the hydroxyl bridged complex

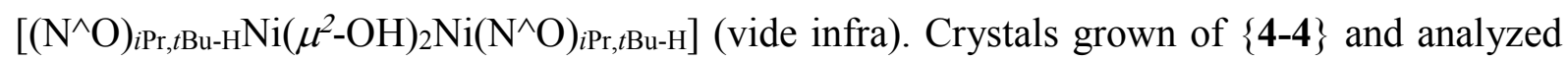
by X-ray diffraction, show a distorted square-planar coordination geometry for $\{\mathbf{4 - 4}\}$, as was previously reported. ${ }^{22 \mathrm{~b}}$ It was stated that this distortion leads to a paramagnetic high-spin complex. However, we were able to record ${ }^{1} \mathrm{H}\left(\mathrm{C}_{6} \mathrm{D}_{6}, \mathrm{RT}, 400 \mathrm{MHz}\right)$ and ${ }^{13} \mathrm{C} \mathrm{NMR}$ spectra characteristic of a diamagnetic compound, which allowed for a full assignment. $\{4-4\}$ shows a paramagnetic behavior when small Lewis bases (e.g. pyridine in $\mathrm{CD}_{2} \mathrm{Cl}_{2}, 25^{\circ} \mathrm{C}$ ) are present. A similar behavoir was previously reported for $\left[\left\{\left(\mathrm{N}^{\wedge} \mathrm{O}\right)_{\mathrm{CF}, \mathrm{I}-\mathrm{I}}\right\}_{2} \mathrm{Ni}\right] .{ }^{11}$ 


\subsection{Experimental Section}

\section{Synthesis of metal-centered radical $\mathrm{Ni}(\mathrm{I})$ complexes}

\section{Synthesis of Chloro tris(triphenylphosphine)Ni(I), [(PPh 3$\left.)_{3} \mathrm{NiCl}\right]$ :}

$\left[\left(\mathrm{PPh}_{3}\right)_{3} \mathrm{NiCl}\right]$ was synthesized by a modified procedure previously reported by Heimbach: ${ }^{16}$

The red suspension of $\left[\mathrm{Ni}\left(\mathrm{PPh}_{3}\right)_{4}\right](1.8 \mathrm{~g}, 16.8 \mathrm{mmol}, 1.1$ equiv. $)$ and $\left[\left(\mathrm{PPh}_{3}\right)_{2} \mathrm{NiCl}_{2}\right](1.0 \mathrm{~g}$, $15.3 \mathrm{mmol}, 1.0$ equiv.) in $\mathrm{Et}_{2} \mathrm{O}(100 \mathrm{~mL})$ was stirred for $5 \mathrm{~h}$ at $\mathrm{RT}$. The resulting yellow suspension was filtered and the yellow solid was washed with $\mathrm{Et}_{2} \mathrm{O}(50 \mathrm{~mL})$ and with pentane $(10 \mathrm{~mL})$. A yellow crystalline powder was obtained and dried under vacuum $(2.3 \mathrm{~g}, 25.5 \mathrm{mmol}$, $83 \%)$.

Elemental analysis revealed a lower carbon content than expected for [( $\left.\left.\mathrm{PPh}_{3}\right)_{3} \mathrm{NiCl}\right]$. This could be due to a varying coordination number $\left(\left[\left(\mathrm{PPh}_{3}\right)_{3} \mathrm{NiCl}\right]\right.$ and $\left.\left[\left(\mathrm{PPh}_{3}\right)_{2} \mathrm{NiCl}\right]\right){ }^{23}$

Crystals suitable for X-ray diffraction were grown from a saturated $\mathrm{Et}_{2} \mathrm{O}$ solution at $-20^{\circ} \mathrm{C}$. $\mathrm{X}$-ray diffraction analysis was performed for analytical reasons to unambiguously identify the obtained compound as $\left[\left(\mathrm{PPh}_{3}\right)_{3} \mathrm{NiCl}\right]$ and is not give herein, since it is almost identical to the structure obtained by Ellis et al. in $2000 .^{23}$

${ }^{1} \mathrm{H}$ NMR (400 MHz, $\left.\mathrm{C}_{6} \mathrm{D}_{6}, \mathrm{RT}\right): \delta=9.56\left(\right.$ br s, $\left.\Delta v_{1 / 2}=87 \mathrm{~Hz}, 2 \mathrm{H}\right), 5.03$ (br s, $\Delta v_{1 / 2}=482 \mathrm{~Hz}$, 2H), 3.35 (br s, $\left.\Delta v_{1 / 2}=55 \mathrm{~Hz}, 1 \mathrm{H}\right) \mathrm{ppm}$.

${ }^{31} \mathrm{P}\left\{{ }^{1} \mathrm{H}\right\}$ NMR (162 MHz, $\left.\mathrm{C}_{6} \mathrm{D}_{6}, \mathrm{RT}\right)$ : no signal was observed between $-200-300 \mathrm{ppm}$.

Anal. Calcd. (\%) for $\mathrm{C}_{54} \mathrm{H}_{45} \mathrm{I}_{2} \mathrm{ClNiP}_{3}$ : C, 73.62; H, 5.15. Found: $\mathrm{C} 71.42, \mathrm{H}, 5.21$.

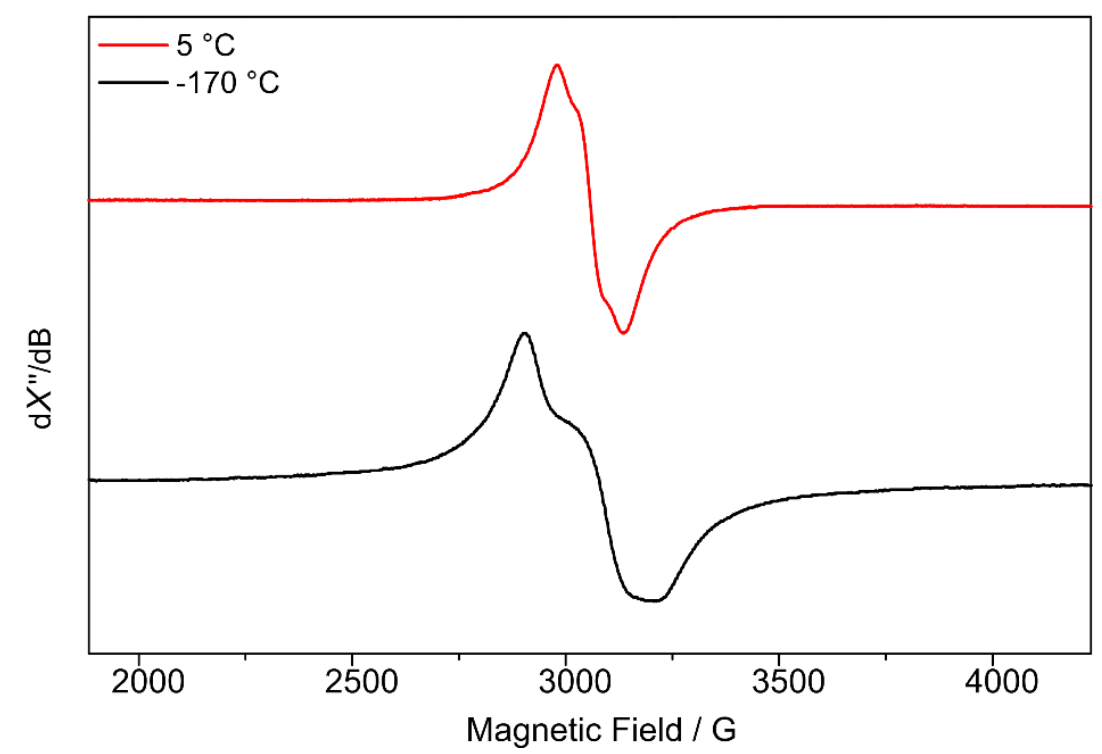

Figure S 3. X-band cw EPR spectra of $\left[\left(\mathrm{PPh}_{3}\right)_{3} \mathrm{NiCl}\right]$ at $5{ }^{\circ} \mathrm{C}(\mathrm{red}, 9.372653 \mathrm{GHz})$ and at $-170{ }^{\circ} \mathrm{C}(\mathrm{black}, 9.373119$ $\mathrm{GHz}$ ) in toluene. Acquisition parameters: $100 \mathrm{kHz}$ modulation frequency, $500 \mathrm{mG}$ modulation, $10 \mathrm{~mW}$ microwave power. 


\section{Synthesis of $\left[\left(\mathrm{N}^{\wedge} \mathrm{O}\right)_{i \mathrm{Pr}, t \mathrm{Bu}-\mathrm{HN}} \mathbf{N}\left(\mathrm{PPh}_{3}\right)_{2}\right]\left(3^{\mathrm{PPh} 3} \mathrm{PPh} 3\right)$ :}

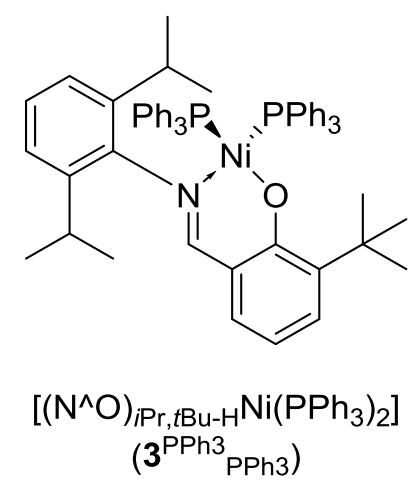

In a Schlenk-flask [( $\left.\left.\mathrm{PPh}_{3}\right)_{3} \mathrm{NiCl}\right](716 \mathrm{mg}, 0.8 \mathrm{mmol}, 1.1$ equiv.) was dissolved in THF (15 $\mathrm{mL})$, the yellow solution was cooled to $-20^{\circ} \mathrm{C}$, and $\left(\mathrm{N}^{\wedge} \mathrm{O}\right)_{i \mathrm{Pr}, t \mathrm{Bu}-\mathrm{HNa}}(243 \mathrm{mg}, 0.7 \mathrm{mmol}, 1.0$ equiv.), dissolved in THF (15 mL), was added dropwise. The solution immediately turned deep red, was stirred for $3.5 \mathrm{~h}$ and was slowly warmed to room temperature. The solvent was removed in vacuo and the residue was extracted with $\mathrm{Et}_{2} \mathrm{O}$, filtered, concentrated and cooled to $-24{ }^{\circ} \mathrm{C}$. Orange/red crystals of $3^{\mathrm{PPh} 3}{ }_{\mathrm{PPh} 3} * \mathrm{Et}_{2} \mathrm{O}$ were obtained and washed with pentane $(390 \mathrm{mg}$, $0.4 \mathrm{mmol}, 56 \%$ yield).

Red crystals suitable for X-ray diffraction were grown from a saturated pentane solution at $-24{ }^{\circ} \mathrm{C}$. $3^{\mathrm{PPh} 3} \mathrm{PPh} 3$ is sparingly soluble in $\mathrm{Et}_{2} \mathrm{O}$ and hardly soluble in pentane. The crystal structure contained one solvent accessible void of $194 \AA^{3}$, containing 43 unassigned electrons in the reflection data. These reflections most likely originate from a disordered pentane molecule, from which the crystals were grown, and these 43 electrons were removed by the PLATON $^{15}$ SQUEEZE routine from the reflection data. The CIF file is deposited at the Cambridge Crystallographic Data Centre with the entry number CCDC 1432842.

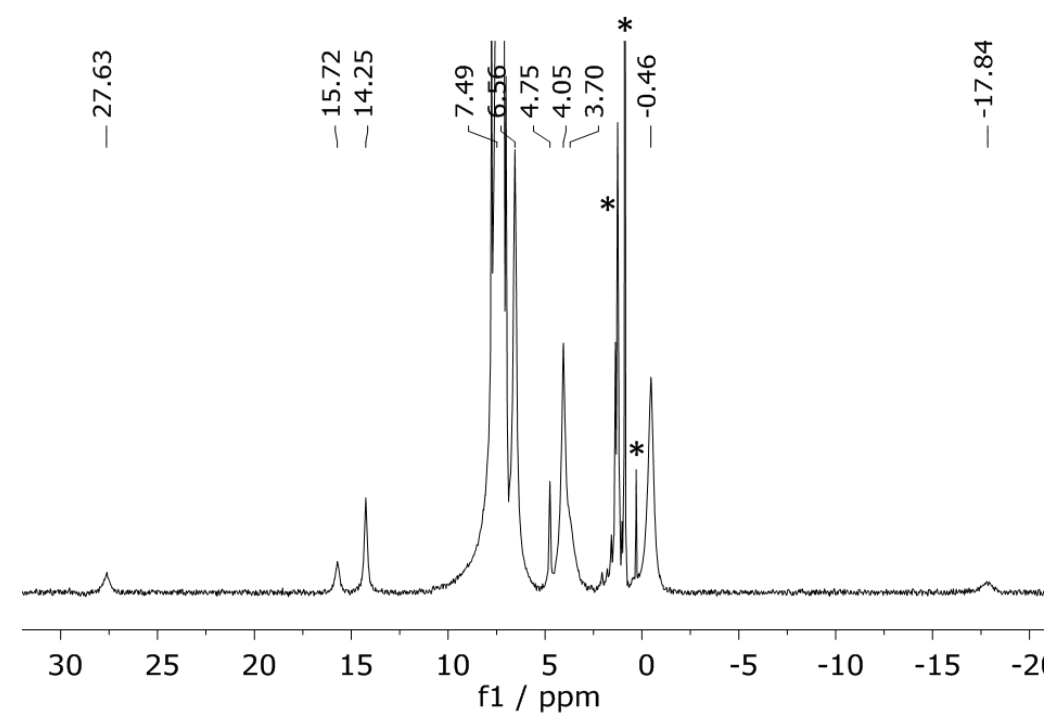

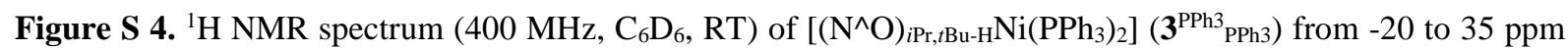
showing the characteristic anisotropic shifted and broadened signals. * marked signals were assigned to impurities in solvent (pentane and silicon grease). 
${ }^{1} \mathrm{H}$ NMR (400 MHz, $\left.\mathrm{C}_{6} \mathrm{D}_{6}, \mathrm{RT}\right): \delta=27.63\left(\right.$ br s, $\left.\Delta v_{1 / 2}=151 \mathrm{~Hz}\right), 15.72\left(\right.$ br s, $\left.\Delta v_{1 / 2}=109 \mathrm{~Hz}\right)$, 14.25 (br s, $\Delta v_{1 / 2}=64 \mathrm{~Hz}$ ) ppm, 7.49 (br s), 6.56 (br s), 4.75 (s, . $\Delta v_{1 / 2}=32 \mathrm{~Hz}$ ), 4.05 (br s, $\Delta v_{1 / 2}=106 \mathrm{~Hz}$ ), 3.70 (br s), -0.46 (br s, $\left.\Delta v_{1 / 2}=130 \mathrm{~Hz}\right),-17.84$ (br s, $\left.\Delta v_{1 / 2}=251 \mathrm{~Hz}\right) \mathrm{ppm}$.

${ }^{31} \mathrm{P}\left\{{ }^{1} \mathrm{H}\right\}$ NMR (162 MHz, $\left.\mathrm{C}_{6} \mathrm{D}_{6}, \mathrm{RT}\right)$ : no signal was observed between -200 - $200 \mathrm{ppm}$.

Anal. Calcd. (\%) for $\mathrm{C}_{59} \mathrm{H}_{60} \mathrm{NNiP}_{2}{ }^{*} \mathrm{Et}_{2} \mathrm{O}$ : C, 76.13; H, 7.10; N, 1.41. Found: C 75.93, H, 6.90; $\mathrm{N}, 1.46$.

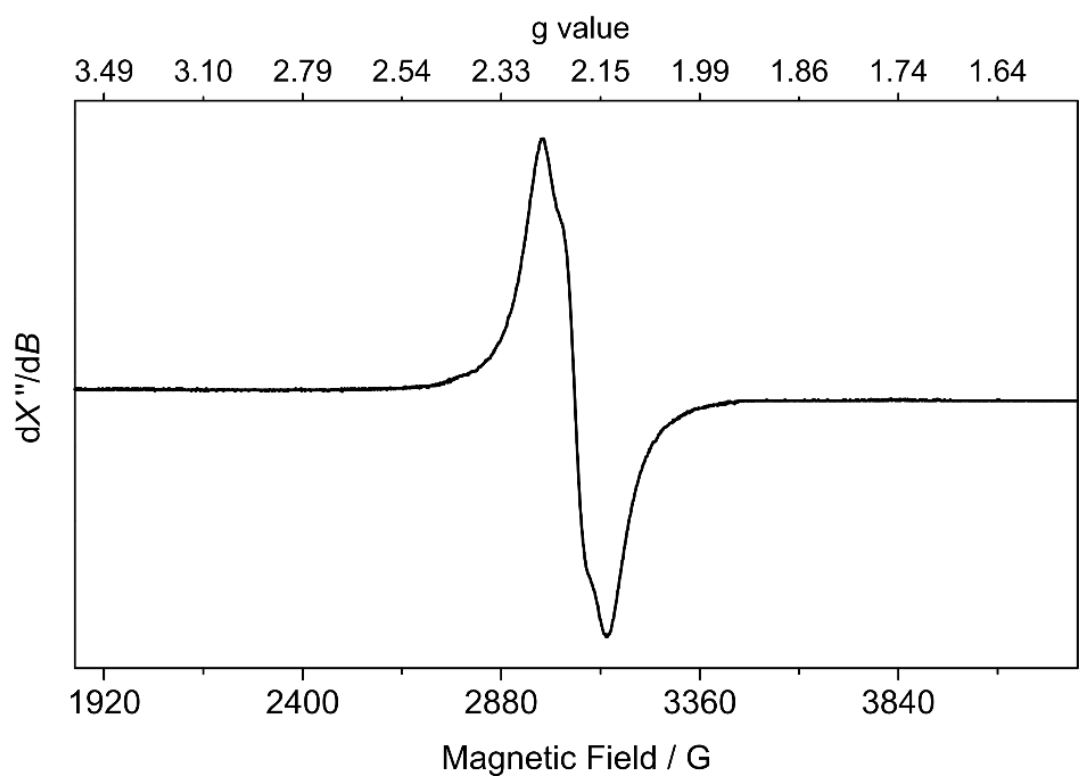

Figure S 5. CW X-band $(v=9.372 \mathrm{GHz})$ EPR spectrum $\left(\mathrm{d} X^{\prime} / \mathrm{dB}\right)$ of $\left[\left(\mathrm{N}^{\wedge} \mathrm{O}\right)_{i \mathrm{Pr}, t \mathrm{Bu}-\mathrm{H}} \mathrm{Ni}(\mathrm{I})\left(\mathrm{PPh}_{3}\right)_{2}\right]$ in toluene at $5{ }^{\circ} \mathrm{C}$. Acquisition parameters: $2500 \mathrm{G}$ sweep, $1000 \mathrm{~s}$ sweep time, $500 \mathrm{mG}$ modulation, 6 Scans, $10.0 \mathrm{~mW}$ microwave power. Simulation of the spectrum gives a $g_{i s o}$-value of 2.20.

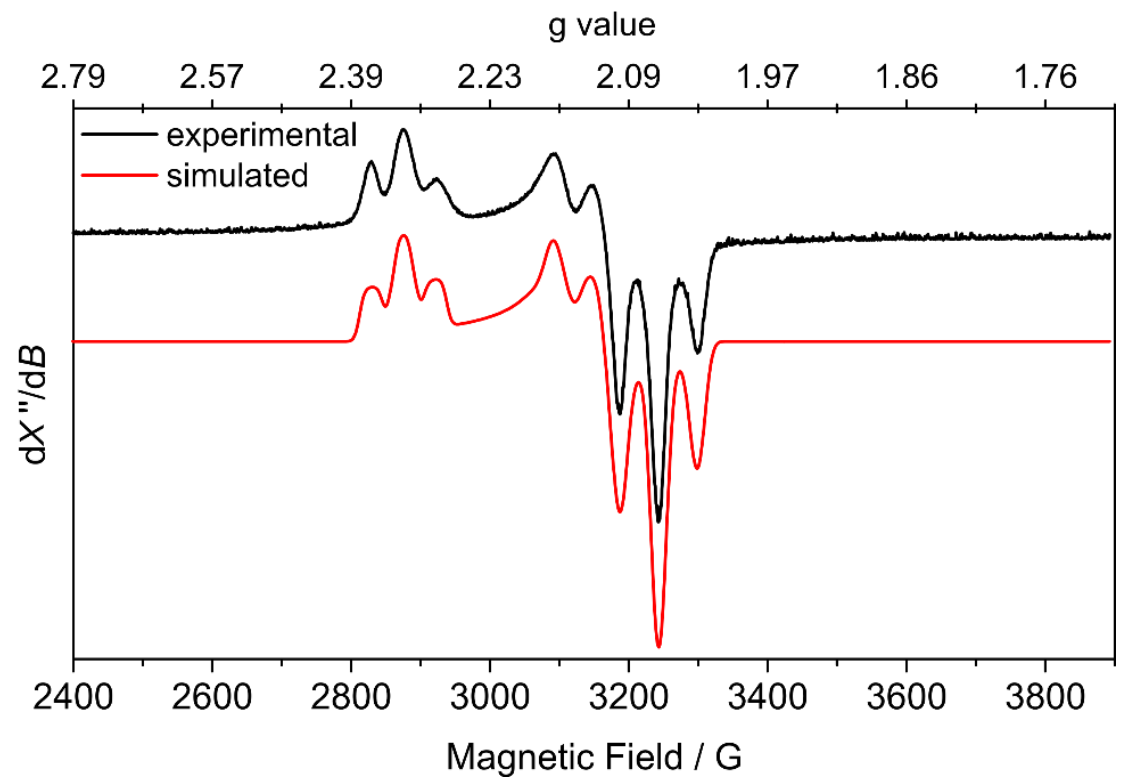

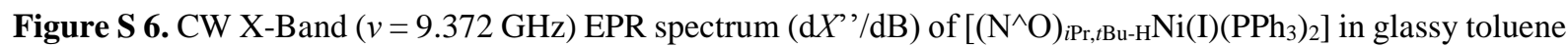
at $-170{ }^{\circ} \mathrm{C}$ (black). Acquisition parameters: $1500 \mathrm{G}$ sweep, $300 \mathrm{~s}$ sweep time, $2000 \mathrm{mG}$ modulation, 5 scans, 10.0 $\mathrm{mW}$ microwave power. The spectrum was simulated (red) by adopting the following values: $g=(2.07,2.12,2.34)$, $H$ strain $=(60,74,52) \mathrm{MHz}, A(\mathrm{~N})=(20,37,37) \mathrm{MHz}, A(\mathrm{P} 1)=(165,196,131) \mathrm{MHz}, A(\mathrm{P} 2)=(156,131,166)$ $\mathrm{MHz}$. 
Stability of $3^{P P h 3}$ PPh3.

In the absence of water and air, complex $3^{\mathrm{PPh} 3}{ }_{\mathrm{PPh} 3}$ shows a high thermal stability (Figure S 7), however, $3^{\mathrm{PPh} 3} \mathrm{PPh} 3$ readily reacts with traces of water and air. The final decomposition product from reaction with water was identified as the bimetallic $\mathrm{OH}^{-}$-bridged complex

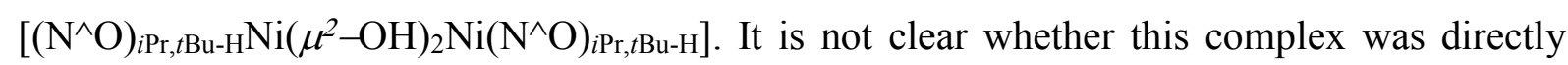
formed by reaction of $3^{\mathrm{PPh} 3}{ }_{\mathrm{PPh} 3}$ with water or if water is accelerating the formation of bischelated complex $\{3-3\}$. Formation of $\left[\left(\mathrm{N}^{\wedge} \mathrm{O}\right)_{i \mathrm{Pr}, t \mathrm{Bu}-\mathrm{H}} \mathrm{Ni}\left(\mu^{2}-\mathrm{OH}\right)_{2} \mathrm{Ni}\left(\mathrm{N}^{\wedge} \mathrm{O}\right)_{i \mathrm{Pr}, t \mathrm{Bu}-\mathrm{H}}\right]$ was also observed in the reaction of $\{\mathbf{3 - 3}\}$ with traces of water. In addition, formation of $\mathrm{OPPh}_{3}$ could be observed when diffusion of water and/or air into the reaction solution was possible (rubber septum sealed reaction vessels).

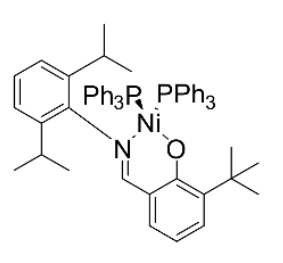

$\left[\left(\mathrm{N}^{\wedge} \mathrm{O}\right)_{\mathrm{Pr}, \text { BBu-H}} \mathrm{Ni}\left(\mathrm{PPh}_{3}\right)_{2}\right]$ (3 $\left.3^{\mathrm{PPh} 3}{ }_{\mathrm{PPh} 3}\right)$ $\mathrm{t}=+96 \mathrm{~h} ; 80^{\circ} \mathrm{C}$

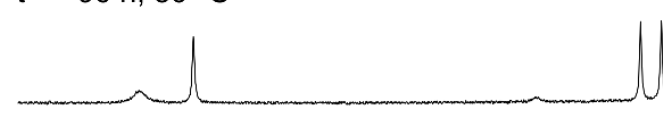

$\mathrm{t}=+96 \mathrm{~h} ; 70^{\circ} \mathrm{C}$

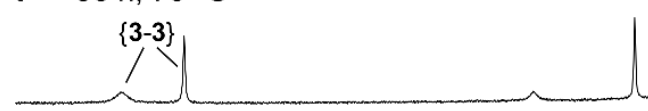

$\mathrm{t}=16 \mathrm{~h} ; 50^{\circ} \mathrm{C}$

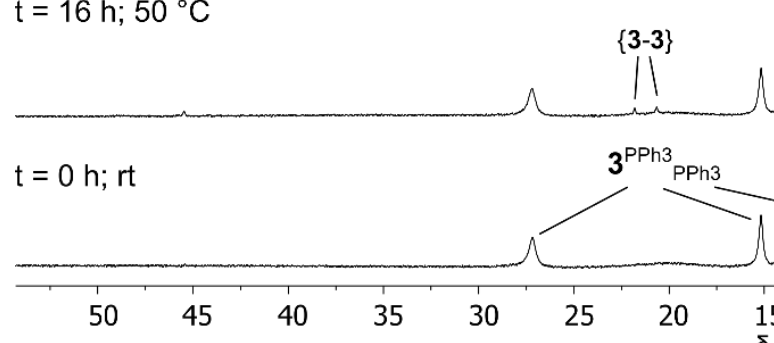

$\{3-3\}$
$\left[\left(\mathrm{N}^{\wedge} \mathrm{O}\right)_{i \mathrm{Pr}, t \mathrm{Bu}-\mathrm{H}} \mathrm{Ni}(\mu-\mathrm{OH})_{2} \mathrm{Ni}\left(\mathrm{N}^{\wedge} \mathrm{O}\right)_{i \mathrm{Pr}, \mathrm{tBu}-\mathrm{H}}\right]$

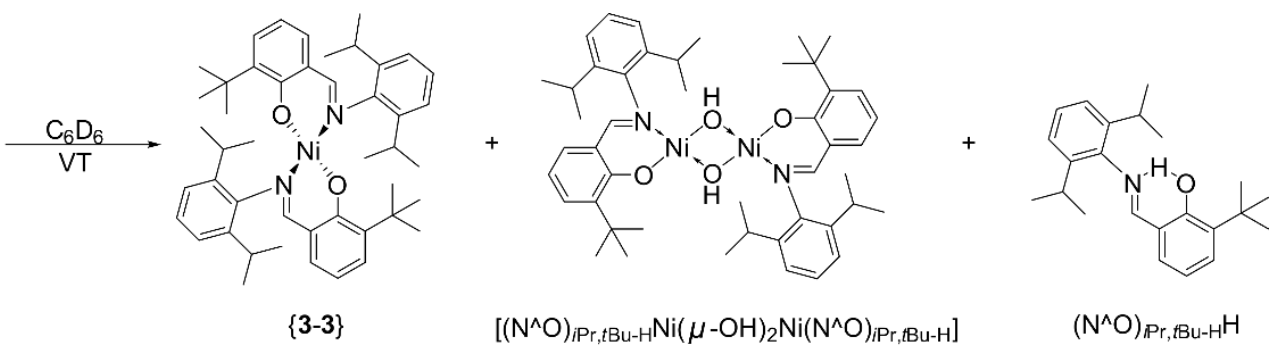

$\left(\mathrm{N}^{\wedge} \mathrm{O}\right)_{\mathbb{P r}, t \mathrm{Bu}-\mathrm{H}^{\mathrm{H}}}$

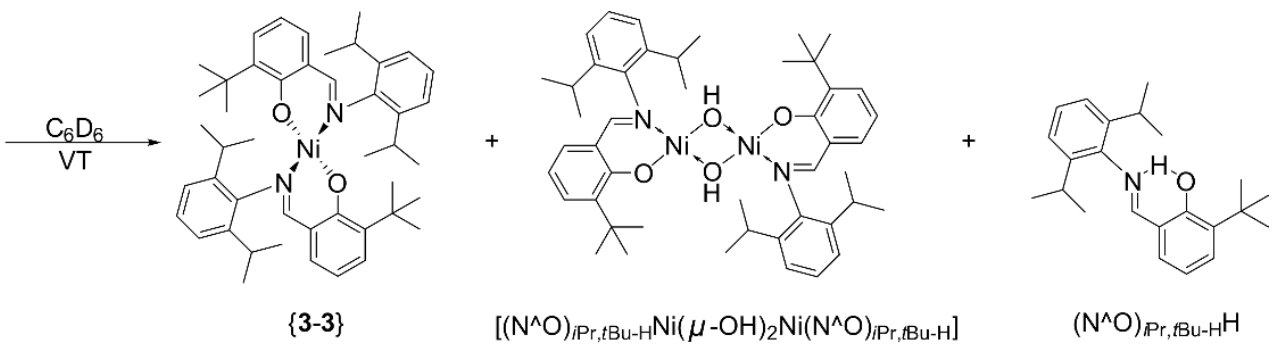

Figure S 7. ${ }^{1} \mathrm{H}$ NMR $\left(400 \mathrm{MHz}, \mathrm{C}_{6} \mathrm{D}_{6}, \mathrm{RT}\right)$ of $\mathbf{3}^{\mathrm{PPh} 3}{ }_{\mathrm{PPh} 3}$ after $0 \mathrm{~h}, 16 \mathrm{~h}$ at $50{ }^{\circ} \mathrm{C}$, additional $96 \mathrm{~h}$ at $70{ }^{\circ} \mathrm{C}$ and additional $96 \mathrm{~h}$ at $80^{\circ} \mathrm{C}$ showing the remarkably high stability of the isolated [Ni(I)] complex in the absence of water and oxygen. The main decomposition product was bis-chelated $\left[\left(\mathrm{N}^{\wedge} \mathrm{O}\right)_{2} \mathrm{Ni}\right]$ complex $\{\mathbf{3 - 3}\}$. Small amounts of a hydroxo-bridged complex were formed from residual water from the solvent. After a prolonged reaction time free protonated ligand was observed $\left.\left(\left(\mathrm{N}^{\wedge} \mathrm{O}\right) \mathrm{H}\right)\right)$. 


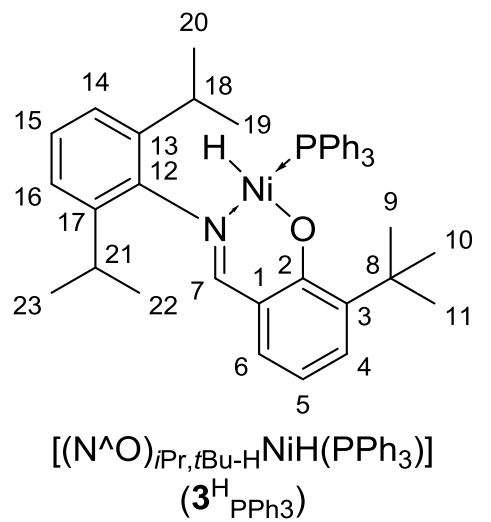

$\left[\left(\mathrm{PPh}_{3}\right)_{2} \mathrm{NiCl}_{2}\right](260 \mathrm{mg}, 0.4 \mathrm{mmol}, 1.3$ equiv. $)$ and $\left[\left(\mathrm{N}^{\wedge} \mathrm{O}\right)_{i \mathrm{Pr}, t \mathrm{Bu}-\mathrm{H}}\right] \mathrm{Na}(100 \mathrm{mg}, 0.3 \mathrm{mmol}, 1.0$ equiv.) was suspended in dichloromethane $(10 \mathrm{~mL})$. After $12 \mathrm{~h}$ the deeply red suspension was filtered over celite, the solvent was evaporated, washed with pentane and extracted with $\mathrm{Et}_{2} \mathrm{O}$. The resulting brown solid of $3^{\mathrm{Cl}}{ }_{\mathrm{PPh} 3}$ was used without further characterization or purification and was containing trace amounts of $\left[\left(\mathrm{PPh}_{3}\right) \mathrm{NiCl}_{2}\right]$, which crystallized from the $\mathrm{Et}_{2} \mathrm{O}$ solution after extraction and was analyzed by X-ray diffraction.

In a J. Young tube $3^{\mathrm{Cl}}{ }_{\mathrm{PPh} 3}\left(10 \mathrm{mg}, 0.016 \mathrm{mmol}, 1\right.$ equiv.) and $\mathrm{NaHB}(\mathrm{OMe})_{3}(6 \mathrm{mg}, 0.047 \mathrm{mmol}$, 3 equiv.) were dissolved in THF- $d_{8}(0.6 \mathrm{~mL})$. The solution immediately turned red, was allowed to react for 20 minutes at room temperature after which quantitative conversion of $3^{\mathrm{Cl}}{ }_{\text {PPh3 }}$ was indicated by ${ }^{1} \mathrm{H}$ NMR spectroscopy along with formation of $3^{\mathrm{H}} \mathrm{PPh}$.

Characteristic NMR resonances of $3^{\mathrm{H}} \mathrm{PPh} 3$ (Figure S 8):

${ }^{1} \mathrm{H}$ NMR (400 MHz, THF- $\left.d_{8}, \mathrm{RT}\right): \delta=7.94\left(\mathrm{~d},{ }^{4} J_{\mathrm{HP}}=9.2 \mathrm{~Hz}, 1 \mathrm{H}, \mathrm{H} 7\right), 6.37\left(\mathrm{vt},{ }^{3} J_{\mathrm{HH}}=7.5 \mathrm{~Hz}\right.$, 1H, H5), 3.83 (hept, $\left.{ }^{3} J_{\mathrm{HH}}=6.8 \mathrm{~Hz}, 2 \mathrm{H}, \mathrm{H} 18 \& \mathrm{H} 21\right), 1.21\left(\mathrm{~d},{ }^{3} J_{\mathrm{HH}}=6.8 \mathrm{~Hz}, 6 \mathrm{H}, \mathrm{H} 19\right.$ \& H22), $1.14\left(\mathrm{~d},{ }^{3} J_{\mathrm{HH}}=6.8 \mathrm{~Hz}, 6 \mathrm{H}, \mathrm{H} 20 \& \mathrm{H} 23\right),-27.56\left(\mathrm{~d},{ }^{2} J_{\mathrm{HP}}=140.2 \mathrm{~Hz}, 1 \mathrm{H},[\mathrm{Ni}-H]\right) \mathrm{ppm}$.

${ }^{31} \mathrm{P}$ NMR (162 MHz, THF- $\left.d_{8}, \mathrm{RT}\right): \delta=26.1\left(\mathrm{brd},{ }^{2} J_{\mathrm{PH}}=140 \mathrm{~Hz}\right) \mathrm{ppm}$. 


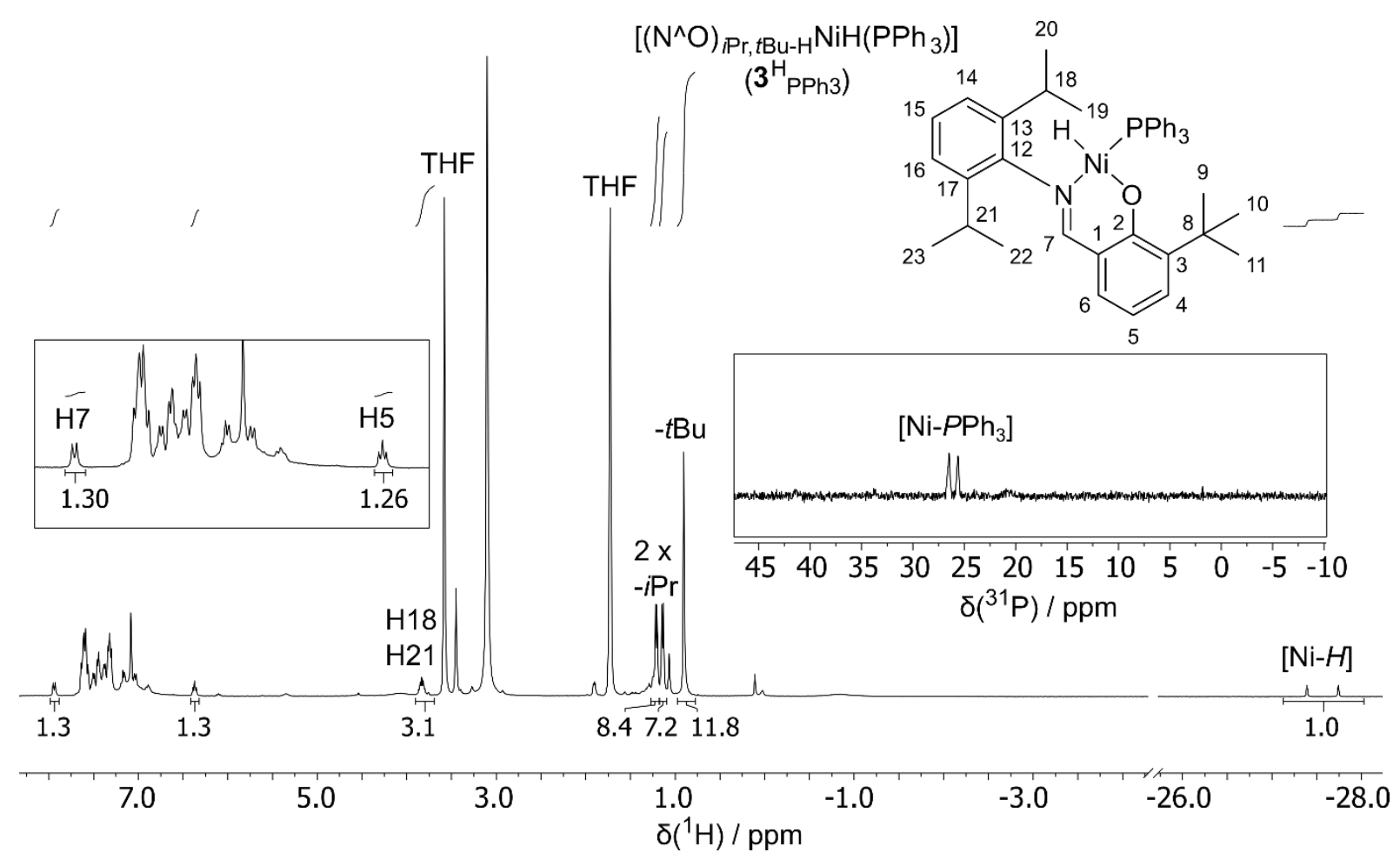

Figure S 8. ${ }^{1} \mathrm{H}$ NMR spectrum $\left(400 \mathrm{MHz}, \mathrm{THF}-d_{8}\right.$, RT) of in-situ formed $\left[\left(\mathrm{N}^{\wedge} \mathrm{O}\right)_{i \mathrm{Pr}, t \mathrm{Bu}-\mathrm{H}} \mathrm{NiH}\left(\mathrm{PPh}_{3}\right)\right]\left(3^{\mathrm{H}} \mathrm{PPh}_{3}\right)$ and the ${ }^{31} \mathrm{P}$ NMR (162 MHz, THF- $\left.d_{8}, \mathrm{RT}\right)$ spectrum is shown as inset (right).

Synthesis of $\left[\left(\mathrm{N}^{\wedge} \mathrm{O}\right)_{i} \mathrm{Pr}, \mathrm{I}-\mathrm{INiMe}\left(\mathrm{Pm}_{\mathrm{Tol}}\right)\right]\left(2^{\mathrm{Me}} \mathrm{Pm}_{\mathrm{T} \text { Tol3 }}\right)$ :

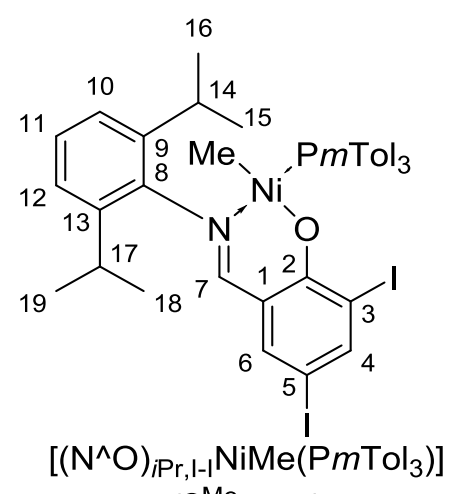

$\left(2^{\mathrm{Me}}{ }_{\mathrm{mTol} 3}\right)$<smiles>Cc1cccc(P(c2cccc(C)c2)c2cccc(C(C)O)c2)c1</smiles>

$\mathrm{PmTOl}_{3}$

In a Schlenk-tube [(tmeda)NiMe 2 (102 mg, $0.5 \mathrm{mmol}, 1.25$ equiv.) was cooled to $-50{ }^{\circ} \mathrm{C}$ and dissolved in THF (50 mL). Slow addition of $\left(\mathrm{N}^{\wedge} \mathrm{O}\right)_{i \mathrm{Pr}, t \mathrm{Bu}-\mathrm{H} H}(213 \mathrm{mg}, 0.4 \mathrm{mmol}, 1.00$ equiv. $)$ in THF $(10 \mathrm{~mL})$ led to an immediate color change to a deep red solution. The temperature was raised to $-20^{\circ} \mathrm{C}$ in $1.5 \mathrm{~h}$ and $\mathrm{P}(m \mathrm{Tol})_{3}(122 \mathrm{mg}, 0.4 \mathrm{mmol}, 1.00$ equiv.) in THF $(5 \mathrm{~mL})$ was added, the solution was warmed to RT and stirred for 30 minutes. After filtration over celite, the red solution was reduced to dryness and washed three times with $\mathrm{MeOH}$ (each time $2 \mathrm{~mL}$ ). The obtained solid was extracted three times with pentane $(5 \mathrm{~mL})$ and cooled to $-24{ }^{\circ} \mathrm{C}$. An orange powder precipitated and was separated from the supernatant solution $(240 \mathrm{mg}$, $0.3 \mathrm{mmol}, 73 \%)$. 
${ }^{1} \mathrm{H}$ NMR (400 MHz, $\left.\mathrm{C}_{6} \mathrm{D}_{6}, \mathrm{RT}\right): \delta=8.08\left(\mathrm{~d},{ }^{4} J_{\mathrm{HH}}=2.3 \mathrm{~Hz}, 1 \mathrm{H}, \mathrm{H} 4\right), 7.96\left(\mathrm{~d},{ }^{3} J_{\mathrm{HP}}=11.0 \mathrm{~Hz}\right.$, $3 \mathrm{H}, \mathrm{H} 21), 7.74$ (vt, $J=8.9 \mathrm{~Hz}, 3 \mathrm{H}, \mathrm{H} 25), 7.45$ (d, $\left.{ }^{4} J_{\mathrm{HP}}=8.2 \mathrm{~Hz}, 1 \mathrm{H}, \mathrm{H} 7\right), 7.10$ (m, 1H, H11), 7.10-7.06 (m, 3H, H6 \& H10 \& H12), 7.35 (vt, $J=7.3 \mathrm{~Hz}, 3 \mathrm{H}, \mathrm{H} 24), 6.90$ (d, ${ }^{3} J_{\mathrm{HH}}=7.7 \mathrm{~Hz}$, $3 \mathrm{H}, \mathrm{H} 23$ ), 4.12 (hept., ${ }^{3} J_{\mathrm{HH}}=7.0 \mathrm{~Hz}, 2 \mathrm{H}, \mathrm{H} 14$ \& 17), 2.01 (s, 9H, H26), 1.34 (d, ${ }^{3} J_{\mathrm{HH}}=6.9 \mathrm{~Hz}$, $6 \mathrm{H}, \mathrm{H} 16 \& \mathrm{H} 19), 1.03\left(\mathrm{~d},{ }^{3} J_{\mathrm{HH}}=6.9 \mathrm{~Hz}, 6 \mathrm{H}, \mathrm{H} 15 \& 18\right),-0.86\left(\mathrm{~d},{ }^{3} J_{\mathrm{HP}}=6.7 \mathrm{~Hz}, 3 \mathrm{H}, \mathrm{Ni}-\mathrm{CH}_{3}\right)$ ppm.

${ }^{13} \mathrm{C}\left\{{ }^{1} \mathrm{H}\right\}$ NMR (101 MHz, C6 $\left.\mathrm{D}_{6}, \mathrm{RT}\right): \delta=164.9$ (C7), $164.0(\mathrm{C} 2), 149.9$ (C4), 149.1 (C8), 142.5 (C6), 141.0 (C9 \& C13), 138.1 (d, $\left.{ }^{3} J_{\mathrm{CP}}=10.3 \mathrm{~Hz}, \mathrm{C} 22\right), 135.8$ (d; $\left.{ }^{2} J_{\mathrm{CP}}=11.7 \mathrm{~Hz}, \mathrm{C} 21\right), 132.5$ $\left(\mathrm{d},{ }^{1} J_{\mathrm{CP}}=43.3 \mathrm{~Hz}, \mathrm{C} 20\right), 131.8\left(\mathrm{~d},{ }^{2} J_{\mathrm{CP}}=9.3 \mathrm{~Hz}, \mathrm{C} 25\right), 131.0\left(\mathrm{~d},{ }^{4} J_{\mathrm{CP}}=1.8 \mathrm{~Hz}, \mathrm{C} 23\right), 128.4$ (C24), 126.7 (C11), 123.7 (C10 \&C12), 120.8 (C1), 97.1 (C3), 72.9 (C5), 28.9 (C14 \& C17), 25.1 (C15 \& C18), 23.3 (C16 \& C19), $21.4(\mathrm{C} 26),-7.7$ (d, $\left.{ }^{2} J_{\mathrm{CP}}=27.3 \mathrm{~Hz}, \mathrm{Ni}-\mathrm{CH}_{3}\right) \mathrm{ppm}$. ${ }^{31} \mathrm{P}\left\{{ }^{1} \mathrm{H}\right\}$ NMR $\left(162 \mathrm{MHz}, \mathrm{C}_{6} \mathrm{D}_{6}, \mathrm{RT}\right): \delta=26.8 \mathrm{ppm}$.

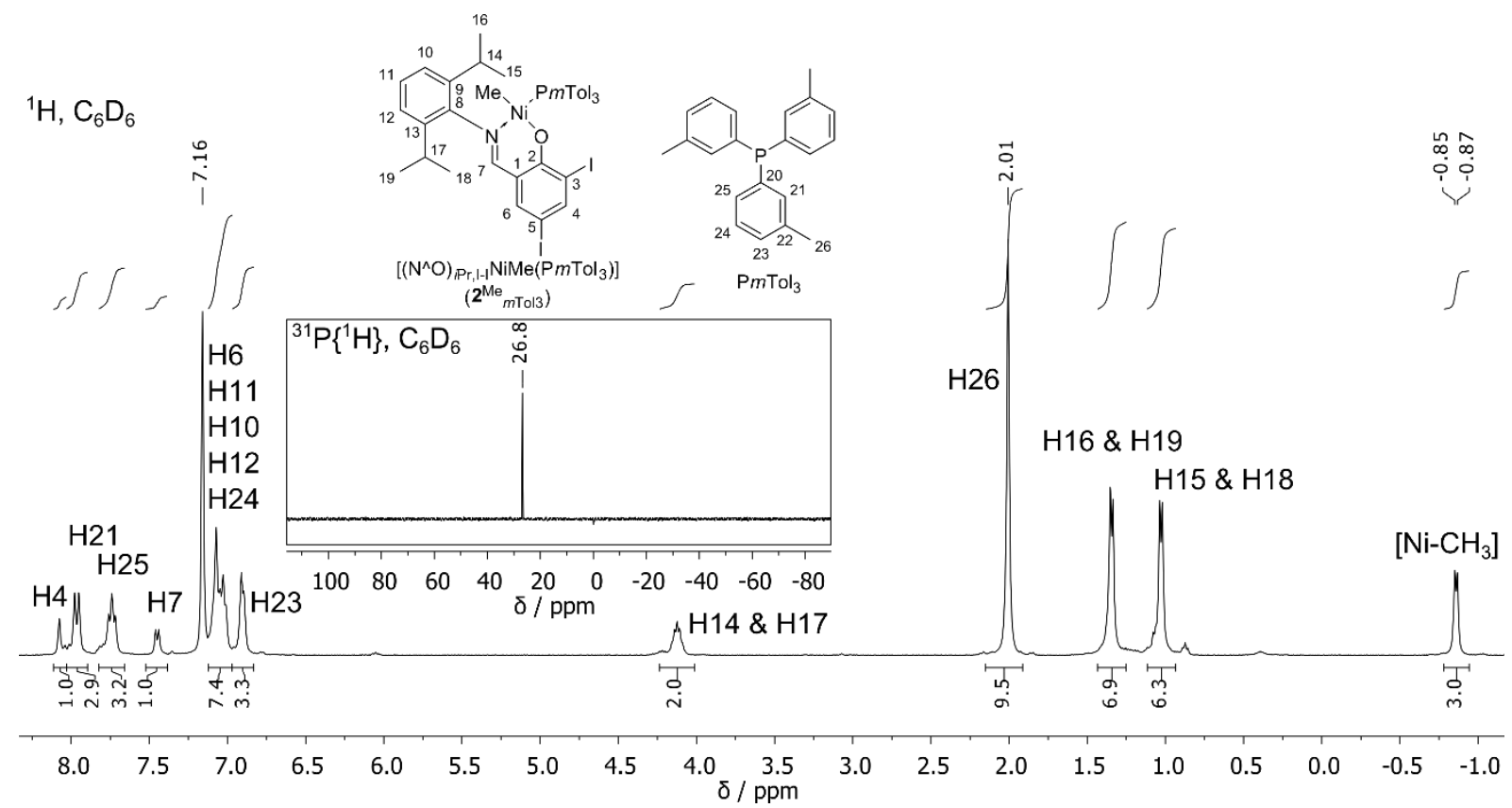

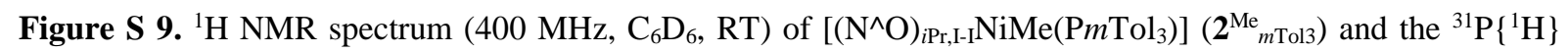
NMR (162 MHz, $\left.\mathrm{C}_{6} \mathrm{D}_{6}, \mathrm{RT}\right)$ as inset. 


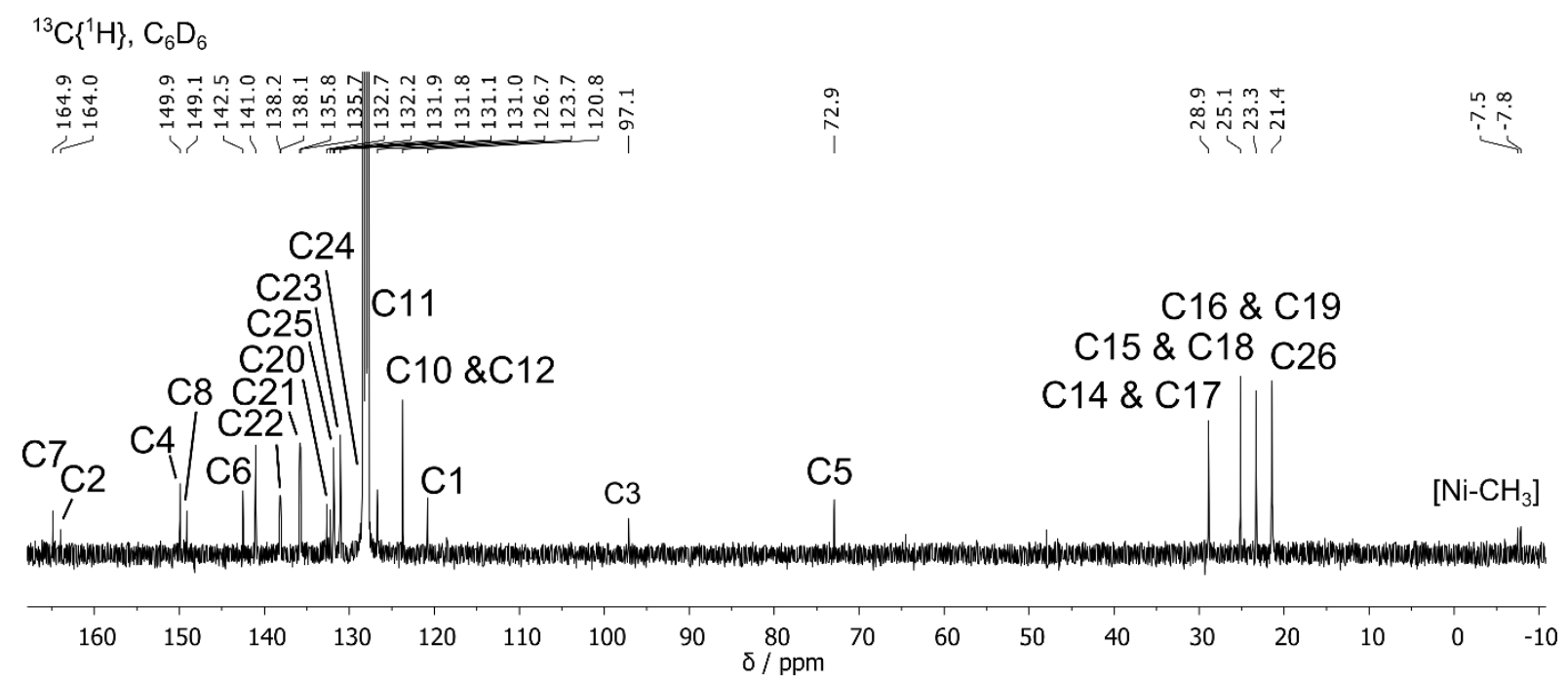

Figure S 10. ${ }^{13} \mathrm{C}\left\{{ }^{1} \mathrm{H}\right]$ NMR spectrum $\left(101 \mathrm{MHz}, \mathrm{C}_{6} \mathrm{D}_{6}, \mathrm{RT}\right)$ of $\left[\left(\mathrm{N}^{\wedge} \mathrm{O}\right)_{i \mathrm{Pr}, \mathrm{II}} \mathrm{Ni}\left(\mathrm{P} m \mathrm{Tol}_{3}\right)\right]\left(2^{\mathrm{Me}}{ }_{m \mathrm{Tol}}\right)$ showing the peak assignment.

Synthesis of $\left[\left(\mathrm{N}^{\wedge} \mathrm{O}\right) \mathrm{CF}, \mathrm{I-I} \mathrm{Ni}(\mathrm{II}) \mathrm{Ph}\left(\mathrm{PPh}_{3}\right)\right]\left(\mathbf{1}^{\mathrm{Ph}}{ }_{\mathrm{PPh}}\right)$.

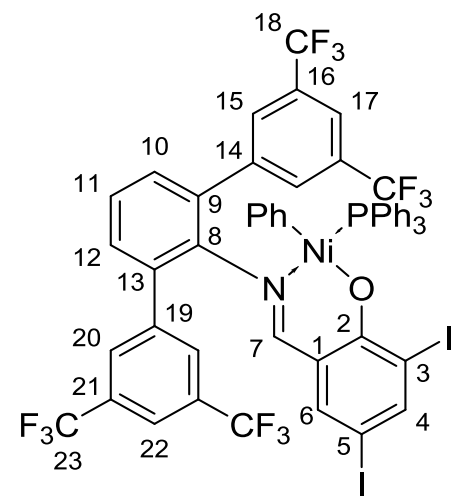

In a Schlenk-tube $\left[\left(\mathrm{PPh}_{3}\right)_{2} \mathrm{Ni}(\mathrm{Cl}) \mathrm{Ph}\right] \cdot(\mathrm{dmf})_{2.8}(489 \mathrm{mg}, 0.50 \mathrm{mmol}, 1$ equiv. $)$ and $\left(\mathrm{N}{ }^{\wedge} \mathrm{O}\right)_{\mathrm{CF}, \mathrm{I}-\mathrm{INa}}$ (495 mg, $0.55 \mathrm{mmol}, 1.1$ equiv.) were dissolved in benzene $(15 \mathrm{~mL})$ and the red/brown solution was stirred for 6 hours at room temperature. The reaction mixture was filtered through a plug of celite and the red/brown solution was evaporated to dryness. The solid was extracted with cold $\mathrm{MeOH}$, reduced under vacuum and the orange precipitate was filtered off and washed with pentane (457 mg, $0.36 \mathrm{mmol}, 72 \%$ ). Crystals suitable for X-ray diffraction were grown from saturated $\mathrm{Et}_{2} \mathrm{O}$ /pentane solution at $-26{ }^{\circ} \mathrm{C}$ (Figure $\mathrm{S} 2$ a). The CIF file is deposited at the Cambridge Crystallographic Data Centre with the entry number CCDC 1432837.

Elemental analysis deviates somewhat from the expected values, what may be due to the high fluorine content of the sample, known to disturb analysis. NMR spectroscopy (Figure S 11) indicates a clean formation of $\mathbf{1}^{\mathrm{Ph}} \mathrm{PPh} 3$.

${ }^{1} \mathrm{H}$ NMR (600 MHz, C6 6 6, RT): $\delta=7.97\left(\mathrm{~d},{ }^{4} J_{\mathrm{HH}}=2.3 \mathrm{~Hz}, 1 \mathrm{H}, \mathrm{H} 4\right), 7.83$ (s, 2H, H17 \& H22), 7.64 (s, 4H, H15 \& H20), 7.15 (m, 6H, P(o-H-Ph)), 7.13 (m, 1H, H7), $7.12\left(\mathrm{~d},{ }^{4} J_{\mathrm{HH}}=2.3 \mathrm{~Hz}\right.$, 1H, H6), $6.96\left(\mathrm{t},{ }^{3} J_{\mathrm{HH}}=7.4 \mathrm{~Hz}, 3 \mathrm{H}, \mathrm{P}(p-H-\mathrm{Ph})\right), 6.88(\mathrm{~m}, 6 \mathrm{H}, \mathrm{P}(m-H-\mathrm{Ph})), 6.72\left(\mathrm{t},{ }^{3} J_{\mathrm{HH}}=7.7\right.$ 
$\mathrm{Hz}, 1 \mathrm{H}, \mathrm{H} 11), 6.61\left(\mathrm{~d},{ }^{3} J_{\mathrm{HH}}=7.7 \mathrm{~Hz}, 2 \mathrm{H}, \mathrm{H} 10 \& \mathrm{H} 12\right), 6.57\left(\mathrm{~d},{ }^{3} J_{\mathrm{HH}}=7.8 \mathrm{~Hz}, 2 \mathrm{H} \mathrm{Ni}-(o-H-\right.$ $\mathrm{Ph})), 6.43\left(\mathrm{t},{ }^{3} J_{\mathrm{HH}}=7.2 \mathrm{~Hz}, 1 \mathrm{H}, \mathrm{Ni}-(p-H-\mathrm{Ph})\right), 6.27$ (vt, $\left.J=7.4 \mathrm{~Hz}, 2 \mathrm{H}, \mathrm{Ni}-(m-H-\mathrm{Ph})\right) \mathrm{ppm}$.

${ }^{13} \mathrm{C}\left\{{ }^{1} \mathrm{H}\right\}$ NMR (151 MHz, C6 $\left.\mathrm{D}_{6}, \mathrm{RT}\right) \delta=168.0$ (C7), 163.3 (C2), 151.0 (C4), 149.1 (C8), 144.9 $\left(\mathrm{d},{ }^{2} J_{\mathrm{CP}}=51.6 \mathrm{~Hz}, \mathrm{Ni}-(i-C-\mathrm{Ph})\right), 142.5$ (C6), 141.5 (C14 \& C19), 137.0 (d, ${ }^{3} J_{\mathrm{CP}}=2.3 \mathrm{~Hz}$, $\mathrm{Ni}-(o-C-\mathrm{Ph})), 134.6\left(\mathrm{~d},{ }^{2} J_{\mathrm{CP}}=10.3 \mathrm{~Hz}, \mathrm{P}(o-C-\mathrm{Ph})\right), 133.4$ (C9 \& C13), 131.3 (br, C15 \& C20), $130.6\left(\mathrm{~d},{ }^{2} J_{\mathrm{CP}}=46.3 \mathrm{~Hz}, \mathrm{P}(i-C-\mathrm{Ph})\right), 130.2\left(\mathrm{~d},{ }^{4} J_{\mathrm{CP}}=1.8 \mathrm{~Hz}, \mathrm{P}(p-C-\mathrm{Ph})\right), 127.9(\mathrm{P}(m-C-\mathrm{Ph})$, $126.4(\mathrm{C} 11), 125.7(\mathrm{Ni}-(m-C-\mathrm{Ph})), 123.6\left(\mathrm{q},{ }^{1} J_{\mathrm{CF}}=273.3 \mathrm{~Hz}, \mathrm{C} 18 \& \mathrm{C} 23\right), 122.1(\mathrm{Ni}-(p-C-\mathrm{Ph}))$, 121.5 (C17 \& C22), 121.4 (d, ${ }^{3} J_{\mathrm{CF}}=4.1 \mathrm{~Hz}, \mathrm{C} 16$ \& C21), 118.7 (C1), 97.1 (C3), 73.8 (C5) ppm.

${ }^{31} \mathrm{P}\left\{{ }^{1} \mathrm{H}\right\}$ NMR $\left(162 \mathrm{MHz}, \mathrm{C}_{6} \mathrm{D}_{6}, \mathrm{RT}\right): \delta=23.2 \mathrm{ppm}$.

${ }^{19} \mathrm{~F}$ NMR (376 MHz, $\left.\mathrm{C}_{6} \mathrm{D}_{6}, \mathrm{RT}\right) \delta=-62.31 \mathrm{ppm}$.

Anal. Calcd. (\%) for $\mathrm{C}_{53} \mathrm{H}_{32} \mathrm{~F}_{12} \mathrm{I}_{2} \mathrm{NNiOP}$ : C, 50.11; H, 2.54; N, 1.10. Found: C 48.50, H, 3.04; N 1.18.

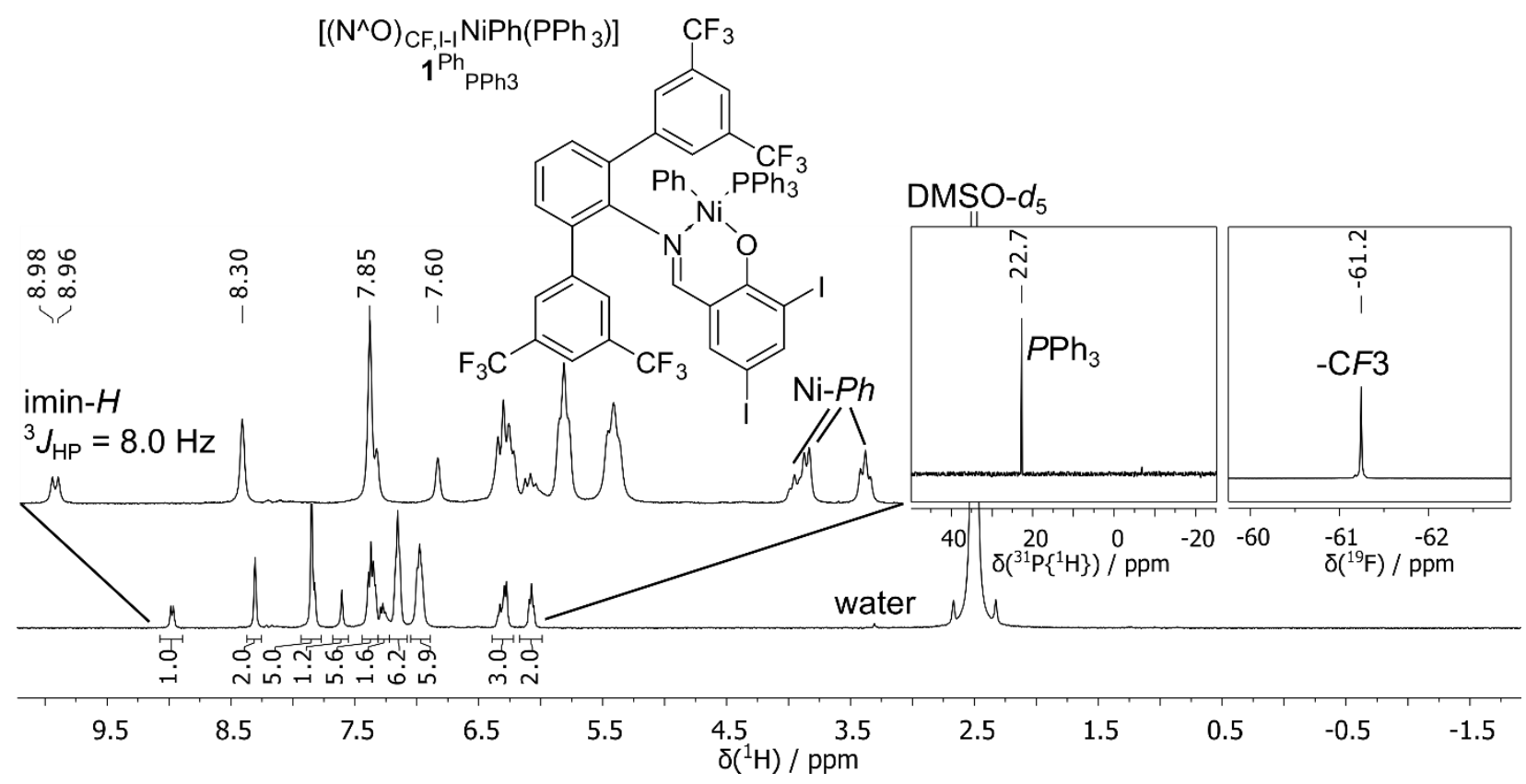

Figure S 11. ${ }^{1} \mathrm{H}(400 \mathrm{MHz}),{ }^{31} \mathrm{P}\left\{{ }^{1} \mathrm{H}\right\}(162 \mathrm{MHz})$ and ${ }^{19} \mathrm{~F}(376 \mathrm{MHz})$ of $\mathbf{1}^{\mathrm{Ph}}{ }_{\mathrm{PPh} 3}$ in DMSO- $d_{6}$ at room temperature. Characteristic resonances are labeled. 


\section{Synthesis of bis-chelated $\left[\left(\mathrm{N}^{\wedge} \mathrm{O}\right)_{2} \mathrm{Ni}\right]$ complexes}

Synthesis of $\left[\left\{\left(\mathrm{N}^{\wedge} \mathrm{O}\right)_{i} \mathrm{Pr}, t \mathrm{Bu}-\mathrm{H}\right\}_{2} \mathrm{Ni}\right](\{3-3\})$ :

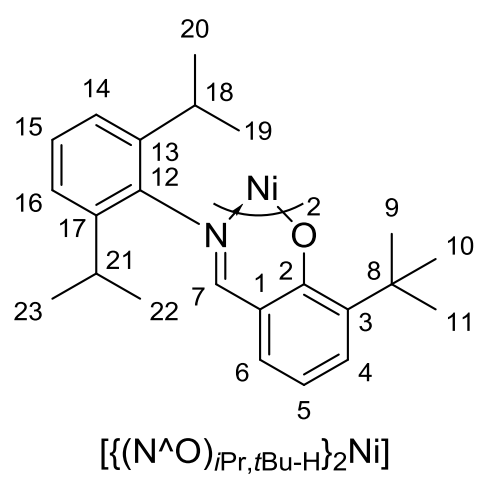

$\{3-3\}$

A different preparation procedure for $\{3-3\}$ was previously reported in literature. ${ }^{22 a}$

In a Schlenk-flask $\left.\left(\mathrm{N}^{\wedge} \mathrm{O}\right)_{i \mathrm{Pr}, t \mathrm{Bu}-\mathrm{HNa}}(206 \mathrm{mg}, 0.57 \mathrm{mmol}, 2.6 \text { equiv.) and [(dmso })_{3} \mathrm{NiCl}_{2}\right](79$ $\mathrm{mg}, 0.22 \mathrm{mmol}, 1.0$ equiv.) was suspended in $\mathrm{Et}_{2} \mathrm{O}(3 \mathrm{~mL})$ and the brown suspension was stirred at room temperature for $17 \mathrm{~h}$, filtered over celite and the solvent was removed in vacuo. The remaining solution was cooled to $-24{ }^{\circ} \mathrm{C}$ and $\{3-3\}$ was obtained as brown crystalline solid in $68 \%$ yield.

Crystals suitable for X-ray diffraction were grown from saturated $\mathrm{Et}_{2} \mathrm{O}$ solution at $-24{ }^{\circ} \mathrm{C}$ (Figure S 12). The CIF file is deposited at the Cambridge Crystallographic Data Centre with the entry number CCDC 1432839.

Elemental composition analysis yields a slightly lower $\mathrm{C}$ content than expected. This might be due to residual dmso resulting from synthesis $\left(\mathrm{C}_{46} \mathrm{H}_{60} \mathrm{~N}_{2} \mathrm{NiO}_{2}{ }^{*} 1 / 7 \mathrm{dmso}: \mathrm{C}, 74.84 ; \mathrm{H}, 8.26 ; \mathrm{N}\right.$, 3.77 in \%).

${ }^{1} \mathrm{H}$ NMR (400 MHz, toluene- $d_{8}$, RT): $\delta=282.84$, 48.67, 45.23, 21.74, 20.60, 13.20, 9.15, 8.87, 5.57, 3.05, -0.91, -8.20, -21.91 ppm.

Anal. Calcd. (\%) for $\mathrm{C}_{46} \mathrm{H}_{60} \mathrm{~N}_{2} \mathrm{NiO}_{2}$ : C, 75.51; H, 8.27; N, 3.83. Found: C 74.85, H, 8.31; N, 3.50 . 


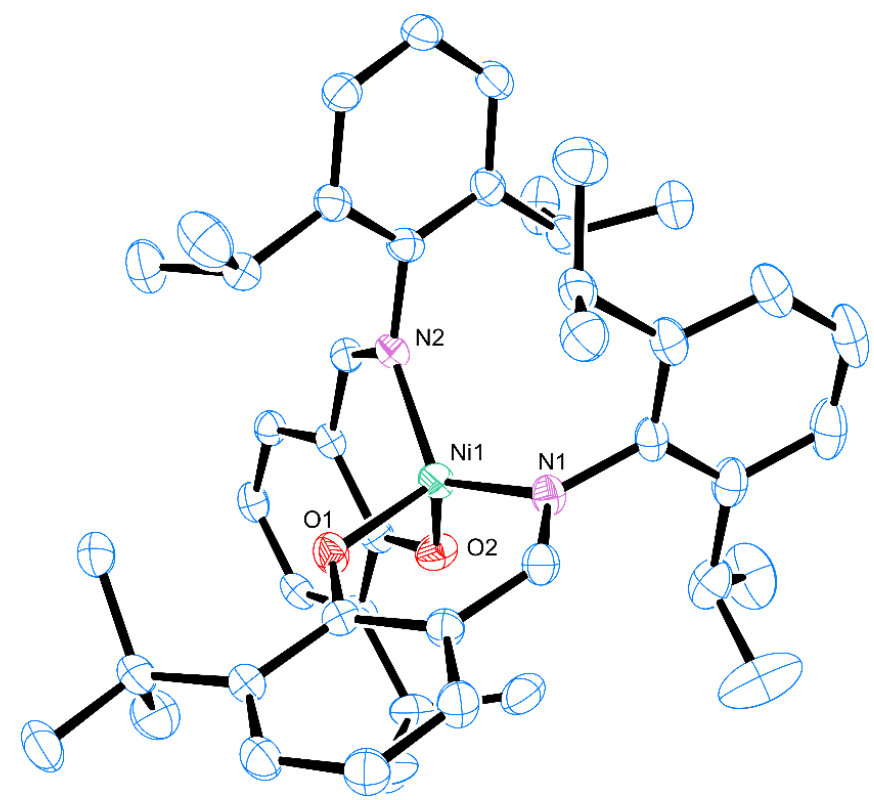

Figure S 12. ORTEP (50\% probability ellipsoids) plot of $\left[\left\{\left(\mathrm{N}^{\wedge} \mathrm{O}\right)_{i \mathrm{Pr}, t \mathrm{Bu}-\mathrm{H}}\right\}_{2} \mathrm{Ni}\right](\{3-3\})$ determined by $\mathrm{X}-$ ray diffraction. Hydrogen atoms are omitted for clarity. Selected bond distances and angles: Ni1-N1 1.964(2) A,Ni1N2 1.973(2) Å, Ni1-O2 1.898(1) Å, Ni1-O2 1.900(1) Å, N1-Ni1-O1 92.57(7) ${ }^{\circ}$, N1-Ni1-N2 126.49(7) ${ }^{\circ}$, N2-Ni1$\mathrm{O} 2$ 91.38(7) ${ }^{\circ}, \mathrm{O} 1-\mathrm{Ni1-O} 2104.82(7)^{\circ}$.

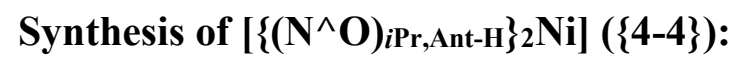

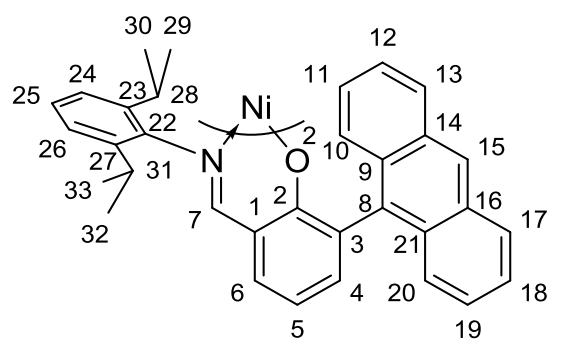

$\left[\left\{\left(\mathrm{N}^{\wedge} \mathrm{O}\right)_{\left.i \mathrm{Pr}, \mathrm{Ant}-\mathrm{H}\}_{2} \mathrm{Ni}\right]}\right.\right.$

(\{4-4\})

Another preparation method for $\{\mathbf{4 - 4}\}$ was previously reported and the resulting complex was characterized as paramagnetic. ${ }^{22 b}$ We herein report a new synthetic approach and a characterization of the resulting diamagnetic complex $\{4-4\}$.

In a Schlenk-tube $\left(\mathrm{N}^{\wedge} \mathrm{O}\right){ }_{i \mathrm{Pr}, \mathrm{Ant}-\mathrm{HNa}}(203 \mathrm{mg}, 0.42 \mathrm{mmol}, 2.6$ equiv. $)$ and [(dmso $\left.)_{3} \mathrm{NiCl}_{2}\right](60 \mathrm{mg}$, $0.16 \mathrm{mmol}, 1$ equiv.) were suspended in THF $(15 \mathrm{~mL})$. The resulting red suspension was stirred for 18 hours at room temperature, filtered over celite and reduced to dryness under vacuum. The resulting red/brown powder was washed with $\mathrm{MeCN}$ and $\{\mathbf{4 - 4}\}$ was obtained in $93 \%$ yield (145 mg, $0.15 \mathrm{mmol}$ ). Crystals suitable for X-ray diffraction were grown from a saturated $\mathrm{MeCN}$ solution at $-20^{\circ} \mathrm{C}$.

The crystal structure was determined by X-ray diffraction to allow for an unambiguous identification of $\{\mathbf{4}-\mathbf{4}\}$, shows a distorted square planar coordination geometry, and is almost identical to previously reported structure by Connor et al. ${ }^{22 b}$ and is not given herein. 
${ }^{1} \mathrm{H}$ NMR (400 MHz, $\left.\mathrm{C}_{6} \mathrm{D}_{6}, \mathrm{RT}\right): \delta=8.17(\mathrm{~s}, 2 \mathrm{H}, \mathrm{H} 15), 7.91\left(\mathrm{~d},{ }^{3} J_{\mathrm{HH}}=8.4 \mathrm{~Hz}, 4 \mathrm{H}, \mathrm{H} 17 \& \mathrm{H} 13\right)$, $7.68\left(\mathrm{dd},{ }^{3} J_{\mathrm{HH}}=8.8 \mathrm{~Hz}{ }^{4} J_{\mathrm{HH}}=1.1 \mathrm{~Hz}, 4 \mathrm{H}, \mathrm{H} 10 \& \mathrm{H} 20\right), 7.34\left(\mathrm{ddd},{ }^{3} J_{\mathrm{HH}}=8.4 \mathrm{~Hz}{ }^{3} J_{\mathrm{HH}}=6.4\right.$ $\left.\mathrm{Hz}^{4} J_{\mathrm{HH}}=1.1 \mathrm{~Hz}, 4 \mathrm{H}, \mathrm{H} 12 \& \mathrm{H} 18\right), 7.23\left(\mathrm{ddd},{ }^{3} J_{\mathrm{HH}}=8.8 \mathrm{~Hz}^{3} J_{\mathrm{HH}}=6.4 \mathrm{~Hz}{ }^{4} J_{\mathrm{HH}}=1.3 \mathrm{~Hz}, 4 \mathrm{H}\right.$, $\mathrm{H} 11$ \& H19), 7.14 (s, 2H, H7), $6.70\left(\mathrm{dd},{ }^{3} J_{\mathrm{HH}}=7.1 \mathrm{~Hz}{ }^{4} J_{\mathrm{HH}}=1.9 \mathrm{~Hz}, \mathrm{H} 2, \mathrm{H} 4\right), 6.64$ (dd, $\left.{ }^{3} J_{\mathrm{HH}}=7.9 \mathrm{~Hz}^{4} J_{\mathrm{HH}}=1.9 \mathrm{~Hz}, \mathrm{H} 2, \mathrm{H} 6\right), 6.21\left(\mathrm{vt},{ }^{3} J_{\mathrm{HH}}=7.4 \mathrm{~Hz}, 2 \mathrm{H}, \mathrm{H} 5\right), 6.02\left(\mathrm{~d},{ }^{3} J_{\mathrm{HH}}=7.6 \mathrm{~Hz}\right.$, 4H, H24 \& H26), 5.89 (t, ${ }^{3} J_{\mathrm{HH}}=7.6 \mathrm{~Hz}, 2 \mathrm{H}, \mathrm{H} 25$ ), 4.37 (hept., ${ }^{3} J_{\mathrm{HH}}=6.9 \mathrm{~Hz}, 4 \mathrm{H}, \mathrm{H} 28 \&$ $\mathrm{H} 31), 1.16\left(\mathrm{~d},{ }^{3} J_{\mathrm{HH}}=6.9 \mathrm{~Hz}, 12 \mathrm{H}, \mathrm{H} 29 \& \mathrm{H} 32\right), 0.97\left(\mathrm{~d},{ }^{3} J_{\mathrm{HH}}=6.9 \mathrm{~Hz}, 12 \mathrm{H}, \mathrm{H} 30\right.$ \& H33) ppm. ${ }^{13} \mathrm{C}\left\{{ }^{1} \mathrm{H}\right\}$ NMR (101 MHz, C6 $\left.\mathrm{D}_{6}, \mathrm{RT}\right): \delta=165.1$ (C7), 161.8 (C2), 145.8 (C22), 139.6 (C4), 139.4 (C23 \& C27), 135.8 (C8), 133.5 (C6), 132.6 (C9 \& C21), 130.6 (C14 \& C16), 128.8 (C10 \& C20), 128.6 (C13 \& C17), 126.1 (C15), 125.1 (C12 6 C18), 124.9 (C25), 124.8 (C11 \& C19), 123.0 (C24 \& C26), 120.9 (C1), 115.1 (C5), 29.9 (C28 \& C31), 24.2 (C29 \& C32), 22.5 (C30 \& C33) ppm.

Anal. Calcd. (\%) for $\mathrm{C}_{66} \mathrm{H}_{60} \mathrm{~N}_{2} \mathrm{NiO}_{2}: \mathrm{C}, 81.56 ; \mathrm{H}, 6.22 ; \mathrm{N}, 2.88$. Found: C 81.36, H, 6.19; N, 3.04 .

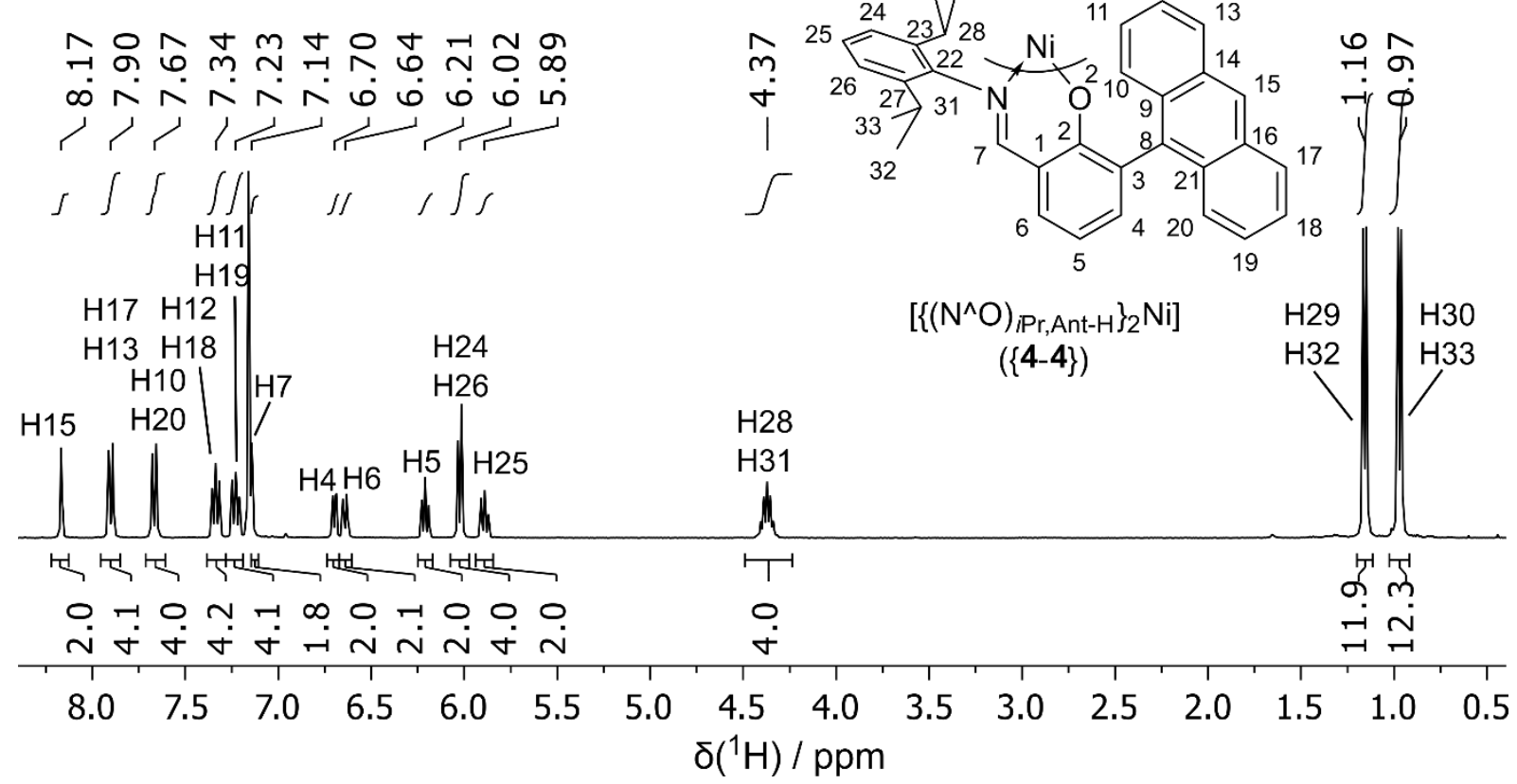

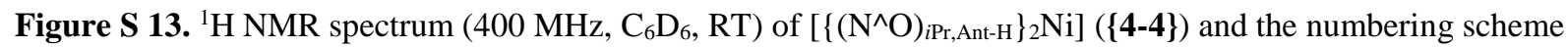
is given as inset. 


\section{Characterization of reaction products.}

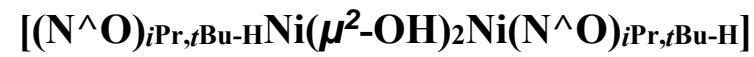

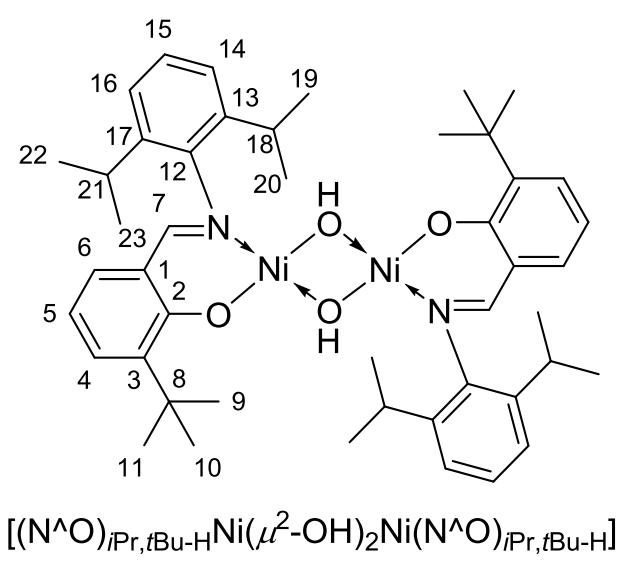

The formation of hydroxyl bridged complex $\left[\left(\mathrm{N}^{\wedge} \mathrm{O}\right)_{i \mathrm{Pr}, t \mathrm{Bu}-\mathrm{H}} \mathrm{Ni}\left(\mu^{2}-\mathrm{OH}\right)_{2} \mathrm{Ni}\left(\mathrm{N}^{\wedge} \mathrm{O}\right)_{i \mathrm{Pr}, t \mathrm{Bu}-\mathrm{H}}\right]$ was observed from Ni(I) complex $3^{\mathrm{PPh} 3}{ }_{\mathrm{PPh} 3}$ as well as from bis-chelated complex $\{\mathbf{3 - 3}\}$ if traces of water were present (e.g. rubber septum sealed NMR tubes). With excess of water $\left(\mathrm{H}_{2} \mathrm{O}\right.$ or $\left.\mathrm{D}_{2} \mathrm{O}\right)$ complex $\left[\left(\mathrm{N}^{\wedge} \mathrm{O}\right)_{i \mathrm{Pr}, t \mathrm{Bu}-\mathrm{H}} \mathrm{Ni}\left(\mu^{2}-\mathrm{OH}\right)_{2} \mathrm{Ni}\left(\mathrm{N}^{\wedge} \mathrm{O}\right)_{i \mathrm{Pr}, t \mathrm{Bu}-\mathrm{H}}\right]$ could not be obtained, instead a pale green

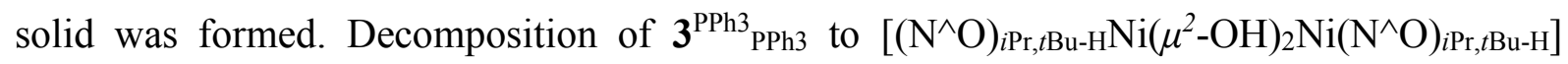
could proceed via $\{3-3\}$ or by direct reaction of $3^{\mathrm{PPh} 3} \mathrm{PPh} 3$ with water. The decomposition of $3^{\mathrm{PPh} 3}{ }_{\mathrm{PPh} 3}$ was significantly faster with traces of water and finally led to formation of

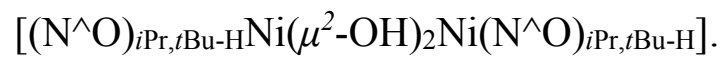

Crystals suitable for X-ray diffraction were obtained from a saturated boiling MeCN solution of $\{3-3\}$ by slowly cooling to room temperature in a rubber septum sealed vial outside a glovebox. Storage outside a glovebox at room temperature yielded brown cuboids of $\left[\left(\mathrm{N}^{\wedge} \mathrm{O}\right)_{i \mathrm{Pr}, t \mathrm{Bu}-\mathrm{H}} \mathrm{Ni}\left(\mu^{2}-\mathrm{OH}\right)_{2} \mathrm{Ni}\left(\mathrm{N}^{\wedge} \mathrm{O}\right)_{i \mathrm{Pr}, t \mathrm{Bu}-\mathrm{H}}\right]$.

$\left[\left(\mathrm{N}^{\wedge} \mathrm{O}\right)_{i \mathrm{Pr}, t \mathrm{Bu}-\mathrm{H}} \mathrm{Ni}\left(\mu^{2}-\mathrm{OH}\right)_{2} \mathrm{Ni}\left(\mathrm{N}^{\wedge} \mathrm{O}\right)_{i \mathrm{Pr}, t \mathrm{Bu}-\mathrm{H}}\right]$ crystalizes in the triclinic space group P-1 and shows a slightly distorted square planar coordination geometry of both Ni-centers (Figure S 14). Protons H47 and H48 ( $\left.\mu^{2}-\mathrm{OH}\right)$ were located in the electron density map after the other hydrogen atoms were treated in a riding model. The CIF file is deposited at the Cambridge Crystallographic Data Centre with the entry number CCDC 1432840. 


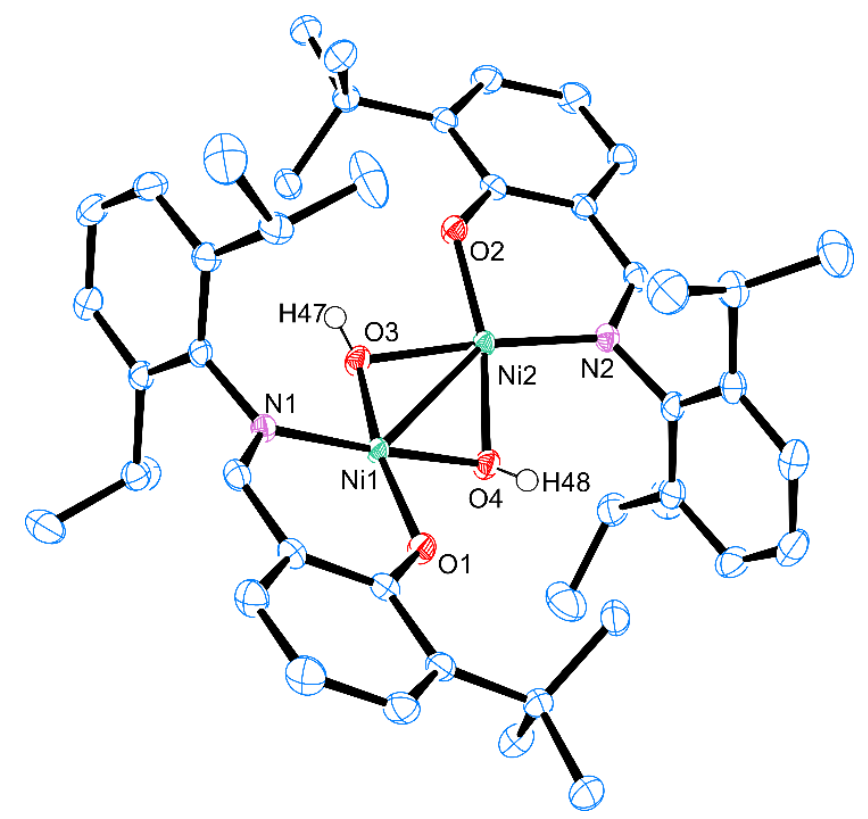

Figure S 14. ORTEP (50\% probability ellipsoids) plot of $\left[\left(\mathrm{N}^{\wedge} \mathrm{O}\right)_{i \mathrm{Pr}, t \mathrm{Bu}-\mathrm{H}} \mathrm{Ni}\left(\mu^{2}-\mathrm{OH}\right)_{2} \mathrm{Ni}\left(\mathrm{N}^{\wedge} \mathrm{O}\right)_{i \mathrm{Pr}, t \mathrm{Bu}-\mathrm{H}]}\right.$ determined by X-ray diffraction. Hydrogen atoms are omitted for clarity beside the hydroxyl protons $\mathrm{H} 47$ and H48. Selected

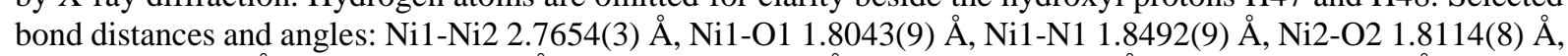

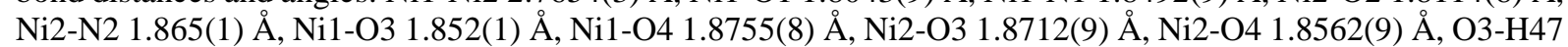

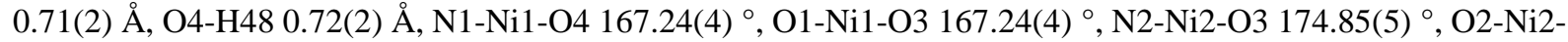
O4 167.28(4) ${ }^{\circ}$.

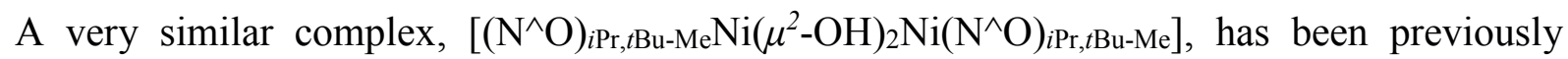
reported, ${ }^{24}$ and was also studied by X-ray crystallography and shows an almost identical coordination geometry.

${ }^{1} \mathrm{H}$ NMR (400 MHz, C6 $\left.\mathrm{D}_{6}, \mathrm{RT}\right) \delta=7.07\left(\mathrm{dd},{ }^{3} J_{\mathrm{HH}}=7.4,{ }^{4} J_{\mathrm{HH}}=1.8 \mathrm{~Hz}, 2 \mathrm{H}, \mathrm{H} 4\right), 7.03-6.93$ $(\mathrm{m}, 2 \mathrm{H}, \mathrm{H} 15), 6.91\left(\mathrm{~d},{ }^{3} \mathrm{~J}_{\mathrm{HH}}=7.6 \mathrm{~Hz}, 4 \mathrm{H}, \mathrm{H} 14\right.$ \& H16), $6.71(\mathrm{~s}, 2 \mathrm{H}, \mathrm{H} 7), 6.62\left(\mathrm{dd},{ }^{3} J_{\mathrm{HH}}=7.6\right.$, $\left.{ }^{4} J_{\mathrm{HH}}=1.8 \mathrm{~Hz}, 2 \mathrm{H}, \mathrm{H} 6\right), 6.39$ (vt, ${ }^{3} J_{\mathrm{HH}}=7.5 \mathrm{~Hz}, 2 \mathrm{H}, \mathrm{H} 5$ ), 4.45 (sept., ${ }^{3} J_{\mathrm{HH}}=6.8 \mathrm{~Hz}, 2 \mathrm{H}, i \operatorname{Pr}$ ), $2.06\left(\mathrm{~d},{ }^{3} J_{\mathrm{HH}}=6.8 \mathrm{~Hz}, 12 \mathrm{H}, t \mathrm{Bu}\right), 1.03(\mathrm{~s}, 18 \mathrm{H}),-8.07(\mathrm{~s}, 2 \mathrm{H},-\mathrm{OH}) \mathrm{ppm}$.

${ }^{13} \mathrm{C}$ NMR (101 MHz, C6 $\left.\mathrm{D}_{6}, \mathrm{RT}\right) \delta=163.9$ (C7), 163.0 (C2), 143.2 (C16), 142.2 (C13 \& C17), 141.0 (C3), 131.5 (C6), 131.2 (C4), 124.0 (C14 \& C16), 120.7 (C1), 115.1 (C5), 35.1 (C8), 29.5 (C9 \& C10 \& C11), 29.0 (C18 \& C21), 24.3 (C19 \& C20 \& C22 \& C23) ppm. C15 could not be located. 


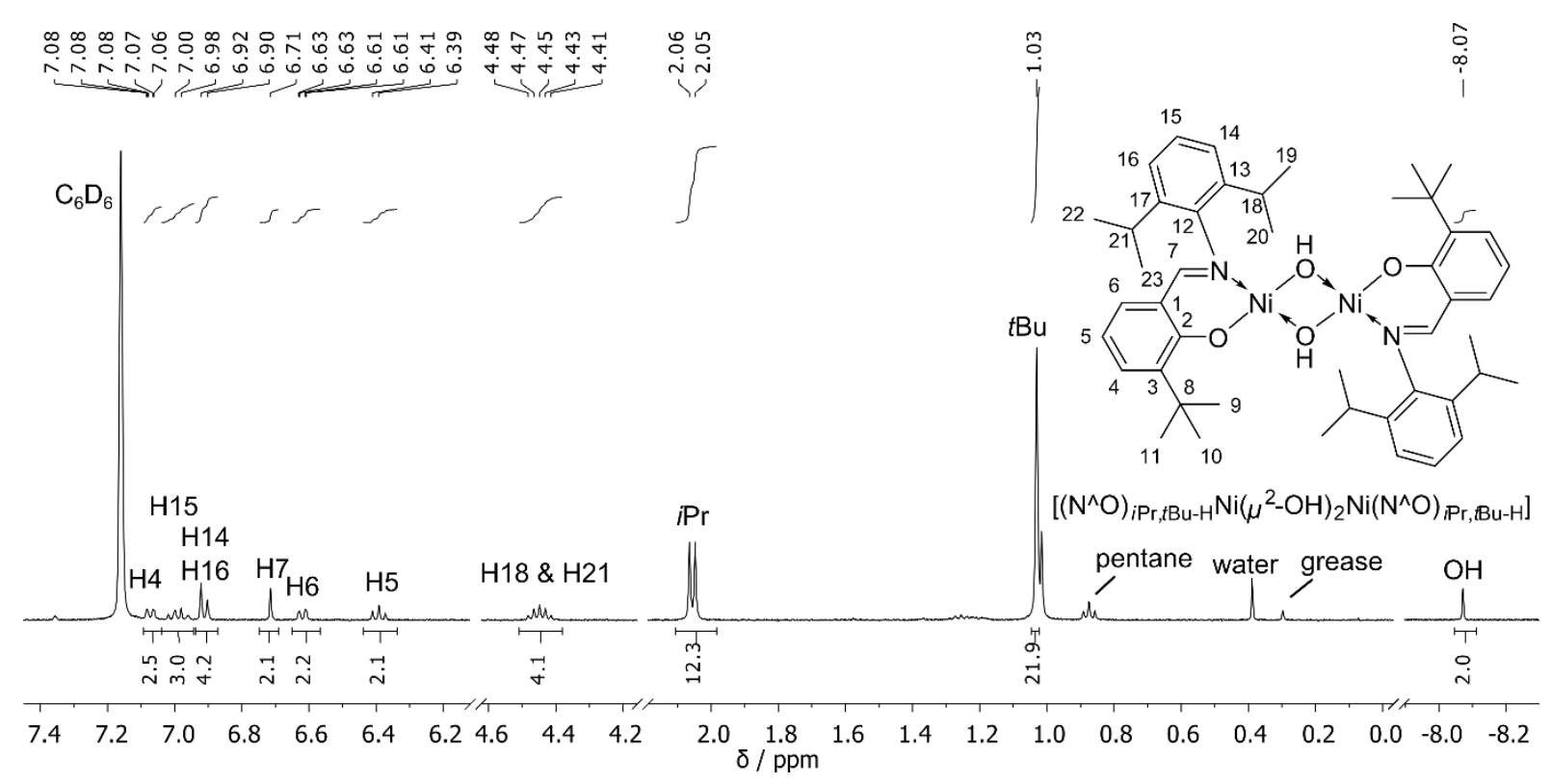

Figure S 15. ${ }^{1} \mathrm{H}$ NMR spectrum $\left(400 \mathrm{MHz}, \mathrm{C}_{6} \mathrm{D}_{6}, \mathrm{RT}\right)$ of $\left[\left(\mathrm{N}^{\wedge} \mathrm{O}\right)_{i \mathrm{Pr}, t \mathrm{Bu}-\mathrm{H}} \mathrm{Ni}\left(\mu^{2}-\mathrm{OH}\right)_{2} \mathrm{Ni}\left(\mathrm{N}^{\wedge} \mathrm{O}\right)_{\left.{ }_{i \mathrm{Pr}, t \mathrm{Bu}-\mathrm{H}}\right]}\right.$. The numbering scheme is given as inset. 


\section{Reactivity of Different Ni Catalyst Precursors toward MMA}

\subsection{Polymerization of MMA initiated with $\left[\left(N^{\wedge} O\right) N i(I I) R\left(P P h_{3}\right)\right]$ complexes}

General procedure for the radical polymerization of MMA alone

The desired amounts of radical initiator and additives were weighted in a Schlenk-tube in a glovebox under $\mathrm{N}_{2}$ atmosphere, sealed with a rubber septum and removed from the glovebox. MMA, and if necessary an additional solvent, was added under inert gas atmosphere. The Schlenk-tube was placed in a preheated aluminum block and the solution was stirred under light exclusion at the desired temperature.

To determine a time-dependent conversion, aliquots were drawn from the reaction mixture, precipitated with an excess of $\mathrm{MeOH}$ and the conversion was determined by the mass of the precipitated polymer.

To quench the polymerization, the reaction mixture was diluted with dichloromethane and the PMMA formed was precipitated from a large excess of stirred $\mathrm{MeOH}$.

The MMA monomer solution was frequently tested, whether PMMA was formed by selfinitiation. In addition, test polymerizations in absence of Ni-complexes (with phosphine) were performed frequently and herein no PMMA formation was observed.

The conversion in all MMA homopolymerizations was below $30 \%$, and therefore a limitation of $M_{\mathrm{n}}$ by a depletion in monomer can be excluded. E.g. for complex $\mathbf{1}^{\mathrm{Ph}}{ }_{\mathrm{PPh} 3}$, elevated polymer yields $(2.8 \%, 6.2 \%$ to $6.8 \%)$ were obtained upon increasing the concentration of $\mathrm{PPh}_{3}$ from 0,3 to 5 equiv. of $\mathrm{PPh}_{3}$, respectively (Table S 2, Entries 1-3).

In the main text it is discussed that the efficiency of the initiation of the radical polymerization is influenced by the initial concentration of supplied [Ni] species. This is concluded from a significant increasing amount of PMMA chains per Ni-center formed with increasing [Ni] concentration (Table S 2, Entries 5-7). Another explanation for this observation could be that chain transfer to the monomer was active. However, chain transfer reactions are first order in the concentration of the radical species, whereas the main chain termination reactions are of second order. Therefore, chain transfer is not more likely when higher concentrations of initial radical initiator are applied. 
Table S 2. MMA homopolymerizations initiated by $\left[\left(\mathrm{N}^{\wedge} \mathrm{O}\right) \mathrm{Ni}(\mathrm{II}) \mathrm{R}\right]$ complexes with or without additions.

\begin{tabular}{|c|c|c|c|c|c|c|c|c|c|}
\hline Entry & Complex & $\begin{array}{l}{[\mathrm{Ni}]} \\
\text { mmol }^{-1} \mathbf{L}^{-1}\end{array}$ & $\begin{array}{l}\text { equiv. } \\
\text { add. }\end{array}$ & $\begin{array}{l}\text { [MMA] }^{\mathrm{a}} \\
/ \mathrm{mol}^{*} \mathbf{l}^{-1}\end{array}$ & $\begin{array}{ll}\text { t } / \\
\text { min }\end{array}$ & $\begin{array}{l}\text { yield } \\
/ \%\end{array}$ & $\begin{array}{l}M_{n}^{b} / 10^{3} \\
\mathrm{~g}^{*} \mathrm{~mol}^{-1}\end{array}$ & $M_{\mathrm{w}} / M_{\mathrm{n}}^{\mathrm{b}}$ & chains/[Ni] ${ }^{\mathrm{c}}$ \\
\hline 1 & $\mathbf{1}^{\mathrm{Ph}}{ }_{\mathrm{PPh}}$ & 0.5 & 0 & 9.389 & 390 & 2.8 & 803 & 1.8 & 0.05 \\
\hline 2 & $\mathbf{1}^{\mathrm{Ph}}{ }_{\mathrm{PPh} 3}$ & 0.5 & $3 \mathrm{PPh}_{3}$ & 9.389 & 440 & 6.2 & 610 & 2.1 & 0.14 \\
\hline 3 & $\mathbf{1}^{\mathrm{Ph}} \mathrm{PPh}_{3}$ & 0.5 & $5 \mathrm{PPh}_{3}$ & 9.389 & 360 & 6.8 & 463 & 2.7 & 0.26 \\
\hline 4 & $\mathbf{1}^{\mathrm{Me}}{ }_{\mathrm{dmso}}$ & 0.5 & $3 \mathrm{PPh}_{3}$ & 9.389 & 261 & 7.2 & 563 & 2.8 & 0.21 \\
\hline 5 & $2^{\mathrm{Ph}} \mathrm{PPh}_{3}$ & 0.5 & $3 \mathrm{PPh}_{3}$ & 9.389 & 440 & 4.3 & 321 & 3.4 & 0.18 \\
\hline 6 & $2^{\mathrm{Ph}}{ }_{\mathrm{PPh} 3}$ & 4.1 & $3 \mathrm{PPh}_{3}$ & 5.633 & 440 & 16.0 & 35 & 2.2 & 0.63 \\
\hline 7 & $2^{\mathrm{Ph}}{ }_{\mathrm{PPh} 3}$ & 8.4 & $3 \mathrm{PPh}_{3}$ & 5.633 & 120 & 27.1 & 12 & 2.0 & 1.52 \\
\hline 8 & $3^{\mathrm{Ph}}{ }_{\mathrm{Ph} 3}$ & 0.5 & 0 & 9.389 & 428 & 1.1 & 270 & 4.3 & 0.06 \\
\hline 9 & $3^{\mathrm{Ph}}{ }_{\mathrm{PPh} 3}$ & 0.5 & $3 \mathrm{PPh}_{3}$ & 9.389 & 428 & 1.8 & 251 & 4.2 & 0.10 \\
\hline
\end{tabular}

Reaction conditions: $70^{\circ} \mathrm{C}$. a Bulk MMA $(9.389 \mathrm{M})$ or toluene was used as a solvent. b Determined by SEC $\left(40{ }^{\circ} \mathrm{C}, \mathrm{THF}\right)$ vs. polystyrene standards. $\left(M_{\mathrm{n}, \mathrm{SEC}}(\mathrm{PMMA})\right)$ c Calculated by (m(PMMA) $\left.\left./ M_{\mathrm{n}, \mathrm{SEC}}(\mathrm{PMMA})\right) / \mathrm{n}(\mathrm{Ni})\right)$.

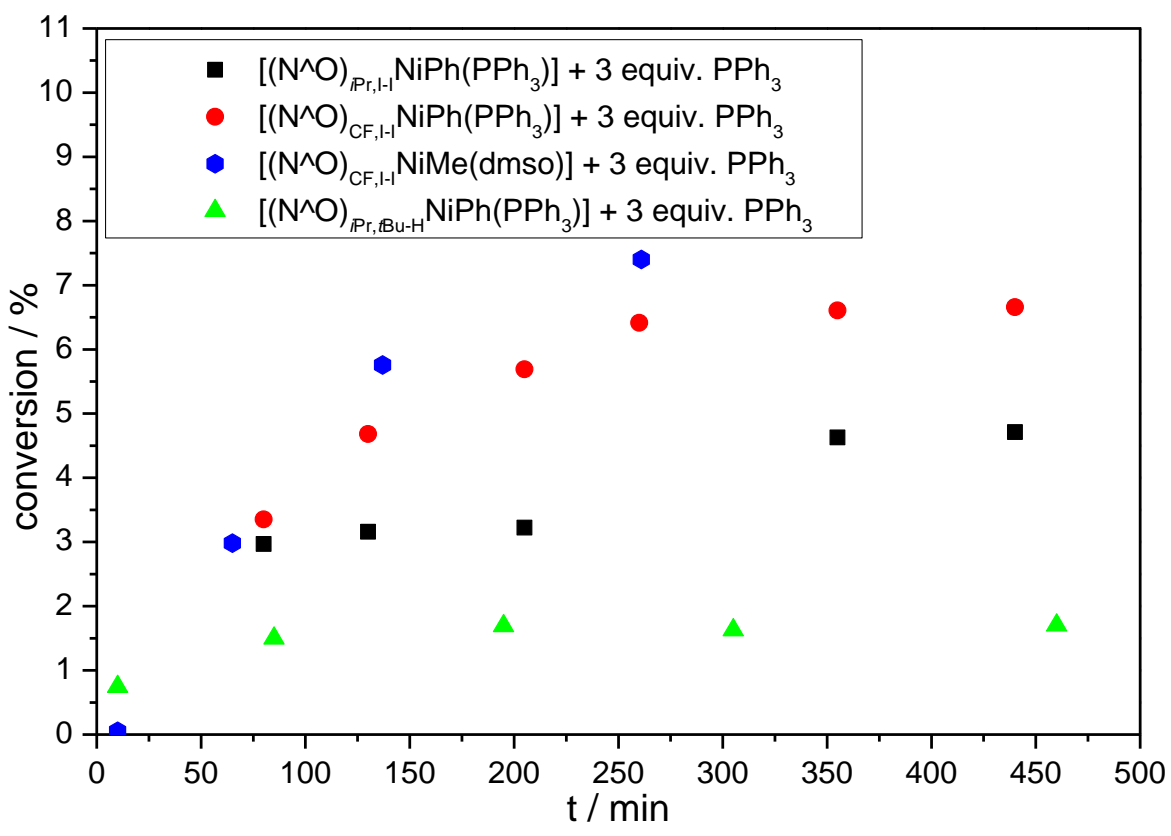

Figure S 16. Time dependent conversions for the radical polymerizations of MMA mediated by $\left[\left(\mathrm{N}^{\wedge} \mathrm{O}\right)_{i \mathrm{Pr}, \mathrm{I}-}\right.$ $\left.{ }_{\mathrm{I}} \mathrm{NiPh}\left(\mathrm{PPh}_{3}\right)\right]$ (black), $\left[\left(\mathrm{N}^{\wedge} \mathrm{O}\right)_{\mathrm{CF}, \mathrm{II}-\mathrm{NiPh}}\left(\mathrm{PPh}_{3}\right)\right] \quad(\mathrm{red}), \quad\left[\left(\mathrm{N}^{\wedge} \mathrm{O}\right)_{\mathrm{CF}, \mathrm{I}-\mathrm{I}} \mathrm{NiMe}(\mathrm{dmso})\right] \quad$ (blue) and $\left[\left(\mathrm{N}^{\wedge} \mathrm{O}\right)_{i \mathrm{Pr}, t \mathrm{Bu}-}\right.$ $\left.{ }_{\mathrm{H}} \mathrm{NiPh}\left(\mathrm{PPh}_{3}\right)\right]$ (green) with additional 3 equiv. $\mathrm{PPh}_{3}$. Reaction conditions: $[\mathrm{MMA}]=9.389 \mathrm{M},[\mathrm{Ni}]=0.5 \mathrm{mM}, \mathrm{T}=$ $70^{\circ} \mathrm{C}$. 


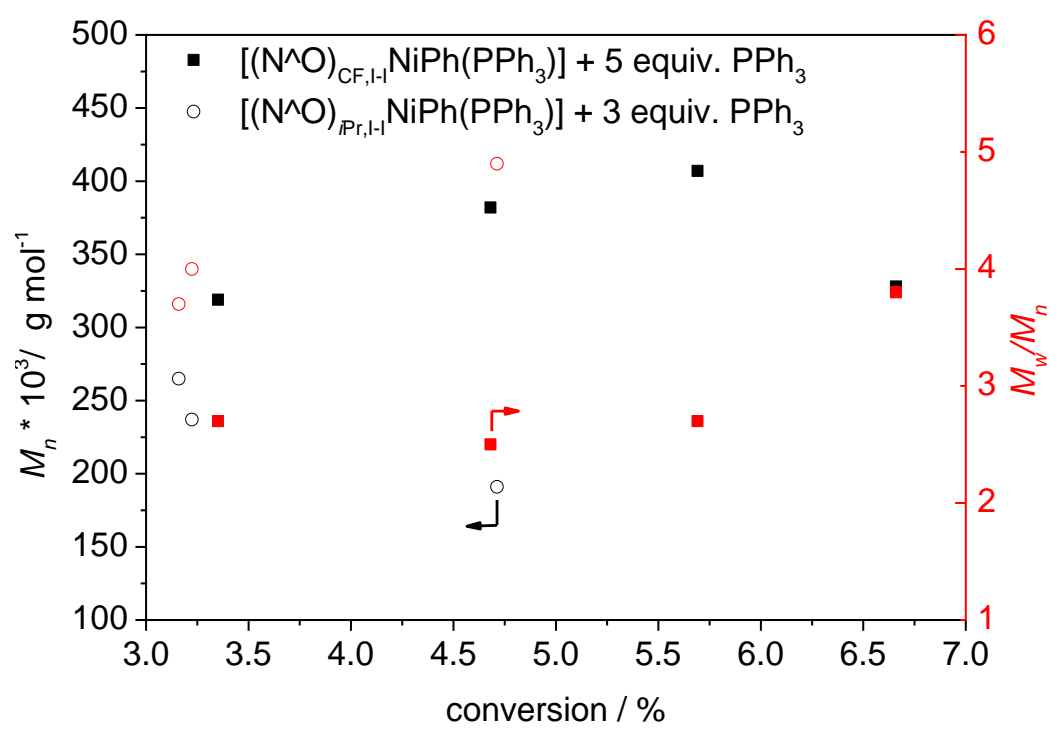

Figure S 17. Conversion dependent $M_{n}$ (black) and $M_{w} / M_{n}$ (red) for the radical polymerization of MMA mediated by $\left[\left(\mathrm{N}^{\wedge} \mathrm{O}\right)_{\mathrm{CF}, \mathrm{I}-\mathrm{I}} \mathrm{NiPh}\left(\mathrm{PPh}_{3}\right)\right]$ (squares) and $\left[\left(\mathrm{N}^{\wedge} \mathrm{O}\right)_{i \mathrm{Pr}, \mathrm{I}-\mathrm{I}} \mathrm{NiPh}\left(\mathrm{PPh}_{3}\right)\right]$ (circles) with additional $\mathrm{PPh}_{3}$. Reaction conditions: $[\mathrm{MMA}]=9.389 \mathrm{M},[\mathrm{Ni}]=0.5 \mathrm{mM}, \mathrm{T}=70^{\circ} \mathrm{C}$. The molecular weights as well as the polydispersity $\left(M_{w} / M_{n}\right)$ indicate that no metal-mediated radical polymerization is active.

\subsection{Microstructure and end-group analysis of PMMA homopolymers.}

a) PMMA initiated with $1^{\mathrm{Ph}}{ }_{\mathrm{PPh} 3}$ :

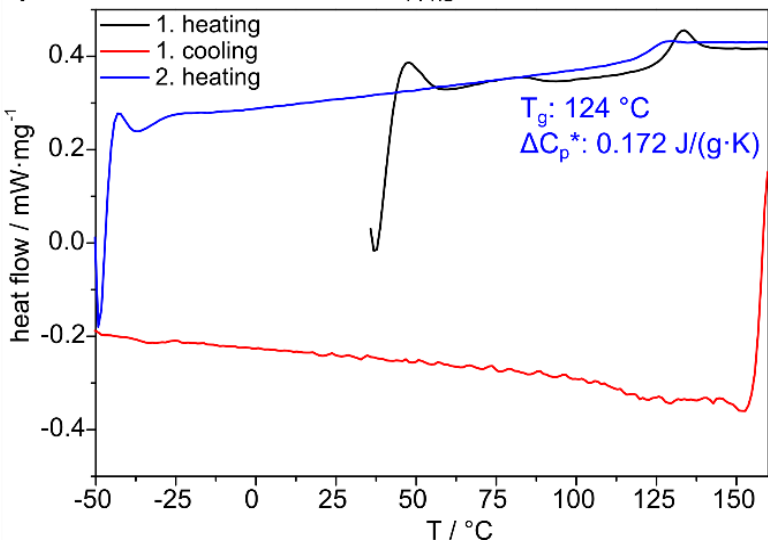

b) PMMA initiated with AIBN:

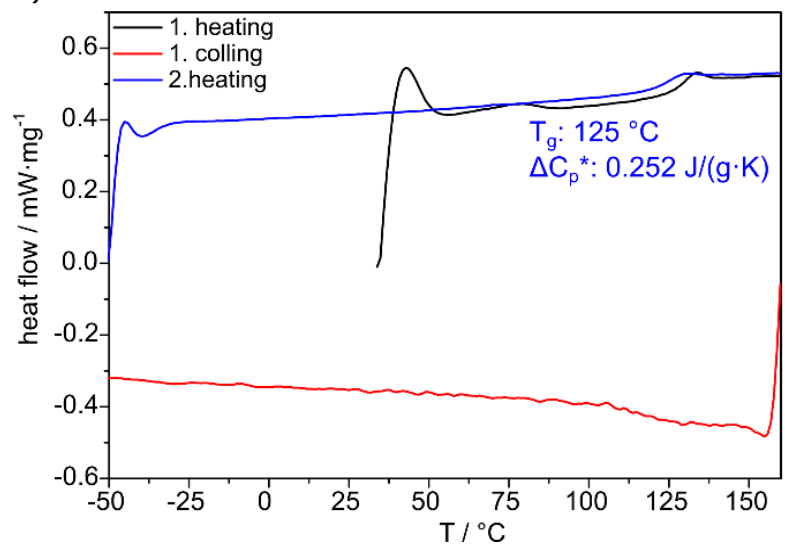

Figure S 18. DSC (10 K· $\mathrm{min}^{-1}$, exotherm down) traces of PMMA formed by radical polymerization of MMA initiated with $\mathbf{1}^{\mathrm{Ph}}{ }_{\mathrm{PPh} 3}$ (a, Table S 2, Entry 1) and by AIBN applying comparable reaction conditions (b).

The tacticity of the PMMA homopolymers was determined by ${ }^{1} \mathrm{H} \mathrm{NMR}$ in $\mathrm{CDCl}_{3}$ or $\mathrm{CD}_{2} \mathrm{Cl}_{2}$ at room temperature by using equation $1 .^{25}$

$$
\% r r=\frac{I_{r r}}{I_{m m}+I_{m r}+I_{r r}}
$$

Leblanc et al. found values of \%rr ranging from $62-66 \%$ for PMMA homopolymers initiated with $2^{\mathrm{Ph}}{ }_{\mathrm{PPh} 3 .}{ }^{26}$ These values are close to the tacticity determined for PMMA homopolymers initiated with $\mathbf{1}^{\mathrm{Ph}}{ }_{\mathrm{PPh} 3}(\% \mathrm{rr}=61 \%$, Figure $\mathrm{S} 19)$ or with $2^{\mathrm{Ph}}{ }_{\mathrm{PPh} 3}$ with additional $\mathrm{PPh}_{3}$ $(\% \mathrm{rr}=61 \%$, Figure S 20) and with PMMA initiated with a classical radical initiator (phenylazo-triphenylmethane, \%rr $=60 \%$, Figure S 21). 
Unsaturated end-groups of PMMA were identified by ${ }^{1} \mathrm{H}$ NMR spectroscopy at 5.45 and 6.15 ppm in $\mathrm{CD}_{2} \mathrm{Cl}_{2}$ (main text, Figure 1). In addition, one equivalent saturated end-groups should be formed by the bimolecular disproportionation reaction. ${ }^{27}$ However, the saturated end-groups are likely to be obscured by the PMMA backbone and we were not able to detect them by NMR spectroscopy.

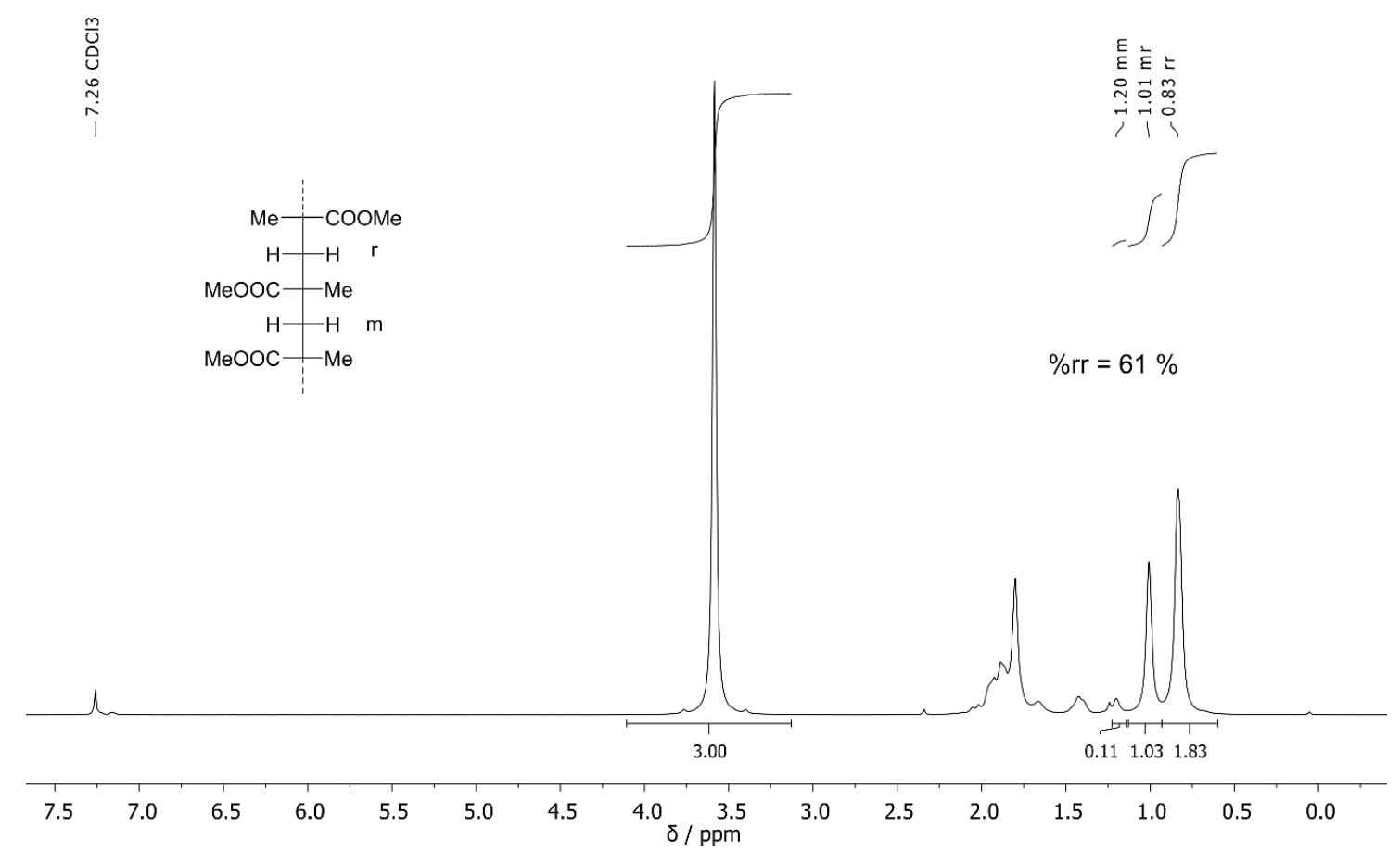

Figure S 19. ${ }^{1} \mathrm{H}$ NMR ( $\left.400 \mathrm{MHz}, \mathrm{CDCl} 3, \mathrm{RT}\right)$ spectrum of PMMA initiated with $\mathbf{1}^{\mathrm{Ph}}{ }_{\mathrm{PPh}}$ (Table $\mathrm{S} 2$, Entry 1$)$. Inset is showing the calculation of \%rr. Reaction conditions: $[\mathrm{Ni}]=0.5 \mathrm{mmol} \cdot \mathrm{L}^{-1},[\mathrm{MMA}]=9.389 \mathrm{~mol} \cdot \mathrm{L}^{-1}, \mathrm{~T}=70{ }^{\circ} \mathrm{C}$.

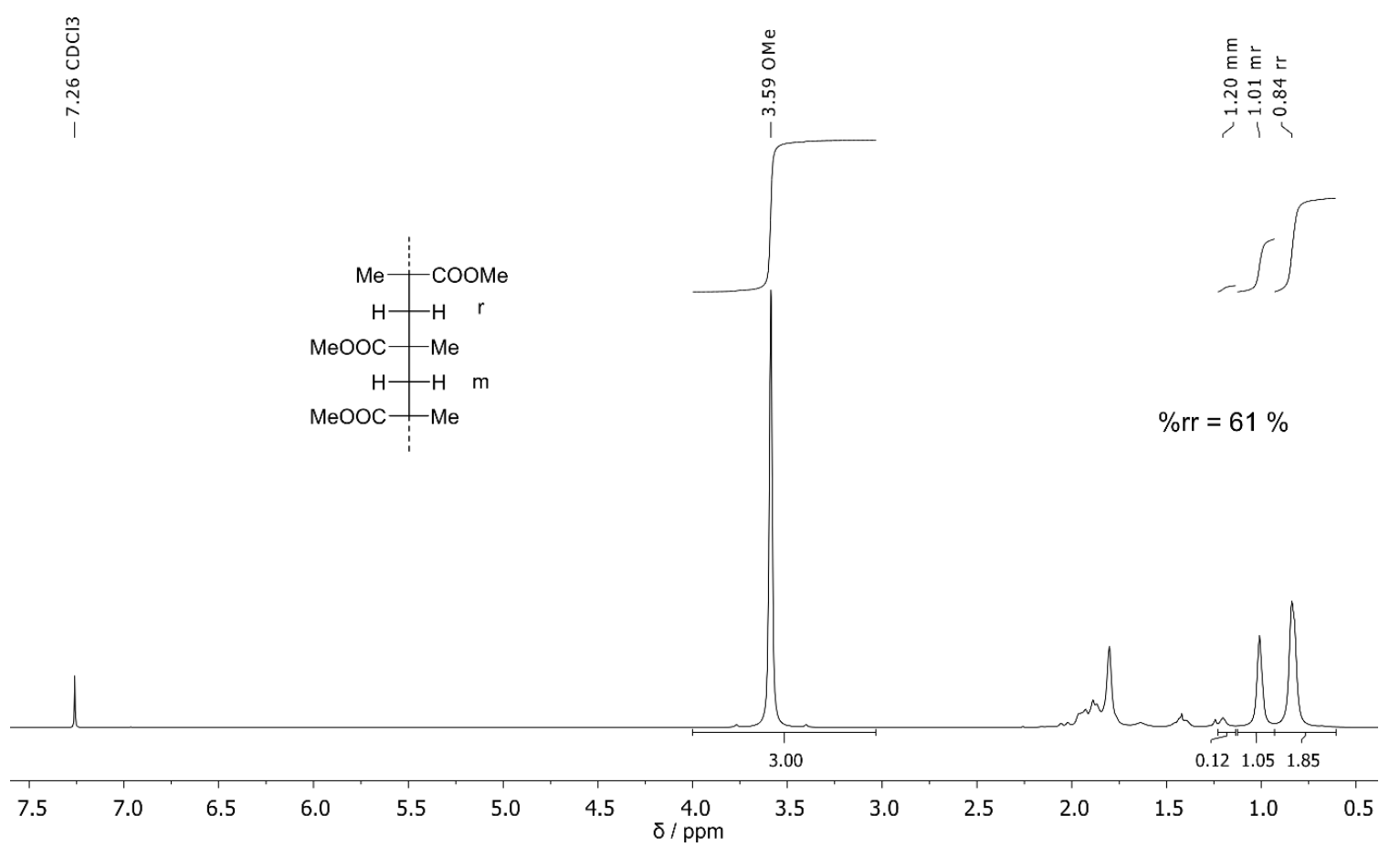

Figure S 20. ${ }^{1} \mathrm{H}-\mathrm{NMR}\left(400 \mathrm{MHz}, \mathrm{CDCl}_{3}, \mathrm{RT}\right)$ spectrum of PMMA initiated with $\mathbf{2}^{\mathrm{Ph}}{ }_{\mathrm{PPh}}, 3$ equiv. $\mathrm{PPh}_{3}$. (Table $\mathrm{S}$ 2, Entry 5). Inset is showing the calculation of \%rr. Reaction conditions: $[\mathrm{Ni}]=0.5 \mathrm{mmol} \cdot \mathrm{L}^{-1}$, [MMA] $=9.389$ $\mathrm{mol} \cdot \mathrm{L}^{-1}, \mathrm{~T}=70{ }^{\circ} \mathrm{C}$. 


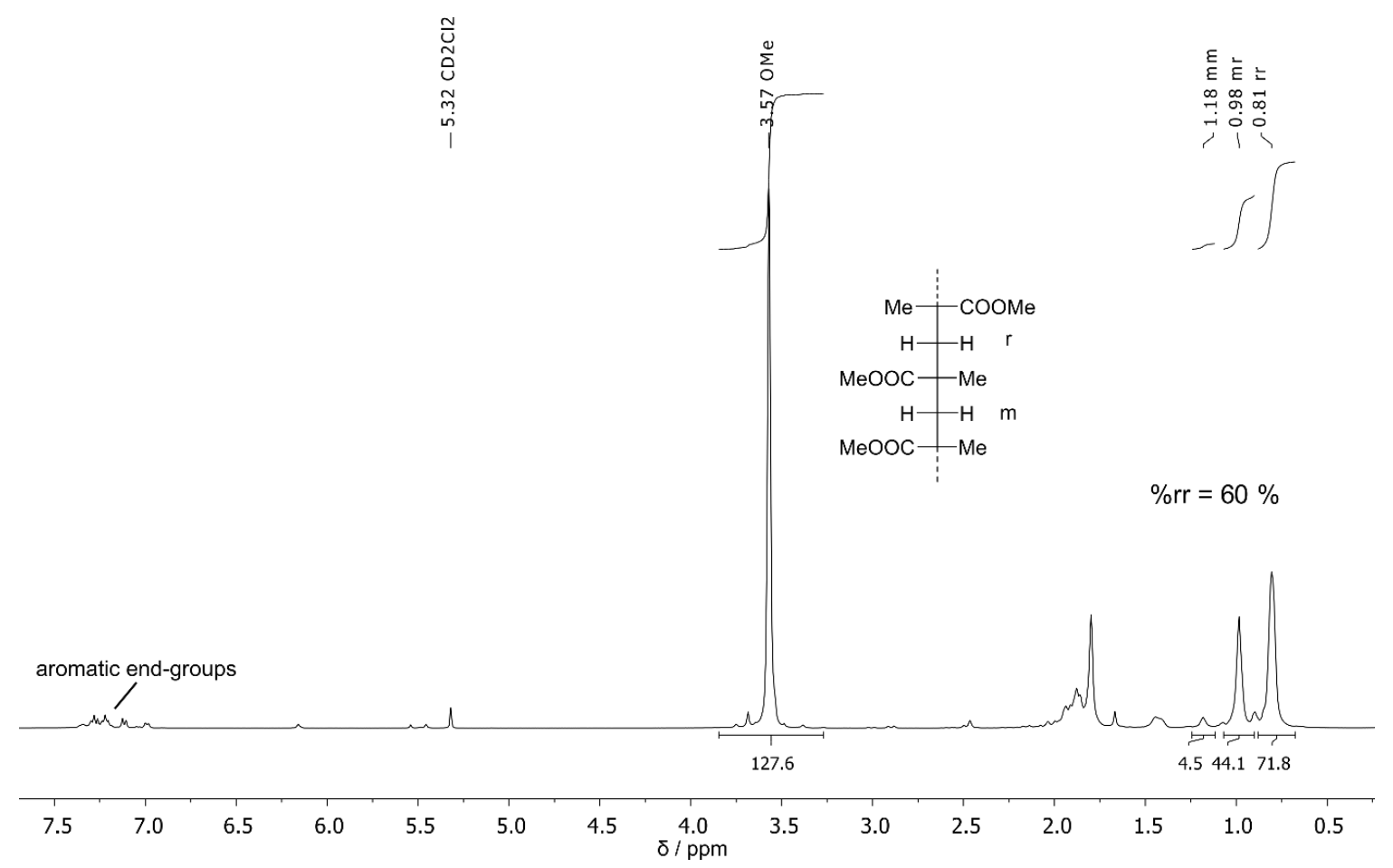

Figure S 21. ${ }^{1} \mathrm{H}-\mathrm{NMR}\left(400 \mathrm{MHz}, \mathrm{CD}_{2} \mathrm{Cl}_{2}\right.$, RT) spectrum PMMA initiated with phenylazo-triphenylmethane. Inset is showing the calculation of \%rr. Reaction conditions: [phenylazo-triphenylmethane] $=0.11 \mathrm{mmol} \cdot \mathrm{L}^{-1},[\mathrm{MMA}]=$ $5.58 \mathrm{~mol} \cdot \mathrm{L}^{-1}, \mathrm{~T}=70^{\circ} \mathrm{C}$.

a)<smiles>CCC(C)(CCC(C)(CC)C(=O)OC)C(=O)OC</smiles>

b) ${ }^{13} \mathrm{C}\left\{{ }^{1} \mathrm{H}\right\}$ NMR of the precipitated polymer:

c) ${ }^{1} \mathrm{H}$ NMR of the $\mathrm{MeOH}$ soluble fraction:

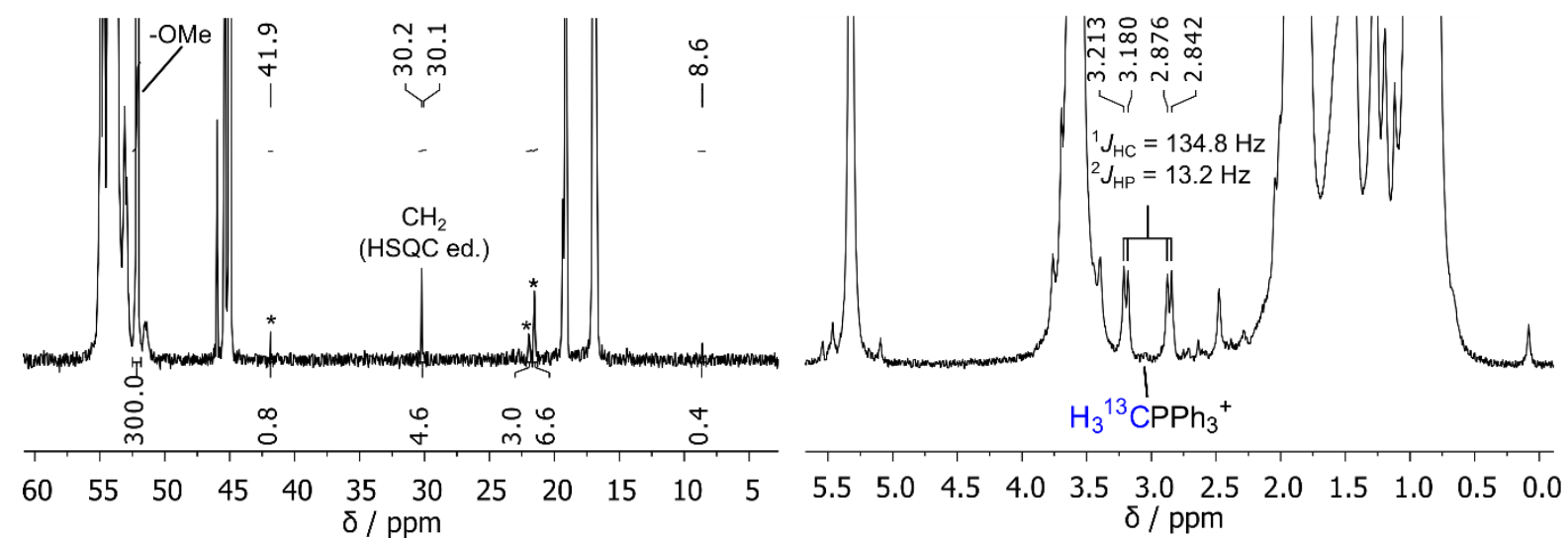

Figure S 22. MMA homopolymerization with ${ }^{13} \mathrm{C}$-labeled catalyst precursor $\mathbf{1}^{13 \mathrm{CH} 3}{ }_{\mathrm{PPh} 3}$ and the estimated endgroups by initiation of the radical polymerization via homolytic bond cleavage of Ni-C (h.b.c.) and the corresponding estimated ${ }^{13} \mathrm{C}$ NMR chemical shifts (a). The ${ }^{13} \mathrm{C}\left\{{ }^{1} \mathrm{H}\right\}$ NMR $\left(600 \mathrm{MHz}, \mathrm{CD}_{2} \mathrm{Cl}_{2}, \mathrm{RT}\right)$ of the precipitated polymer $\left(30 \cdot 10^{3} \mathrm{~g} \cdot \mathrm{mol}^{-1}\right.$, from pentane and afterwards from $\left.\mathrm{MeOH}\right)$ is shown in (b). No transferred ${ }^{13} \mathrm{C}$ label from $\mathbf{1}^{13 \mathrm{CH} 3} \mathrm{PPh} 3$ to the polymer could be detected. Peaks labeled with an $*$ were also observed when polymerizations were initiated with [Ni-Ph] complexes. In the ${ }^{1} \mathrm{H}$ NMR (400 MHz, $\left.\mathrm{CD}_{2} \mathrm{Cl}_{2}, \mathrm{RT}\right)$ spectrum of the $\mathrm{MeOH}$ soluble fraction a significant amount of ${ }^{13} \mathrm{C}$ labeled reductive elimination product to labile phosphine ligand $\left(\mathrm{H}_{3}{ }^{13} \mathrm{CPPh}_{3}{ }^{+}, 1.1 \mu \mathrm{mol}, 3 \mathrm{ppm}\right.$, dd) was detected.

Quantification of the ${ }^{13} \mathrm{C}$ labeled compounds which were separated from the unlabeled PMMA (1.4 $\mu \mathrm{mol}$, Figure S 22) showed, that from the initial $19.0 \mu \mathrm{mol}$ of $\mathbf{1}^{13 \mathrm{CH}^{3}{ }_{\mathrm{Phh} 3}} 7.9 \mu \mathrm{mol}$ could be 
recovered unmodified after polymerization, while $1.1 \mu$ mol of $\mathrm{H}_{3}{ }^{13} \mathrm{CPPh}_{3}{ }^{+}$was formed (Figure S 22c). The remaining $10 \mu \mathrm{mol}$ of ${ }^{13} \mathrm{CH}_{3}$ label could not be recovered and were likely lost as gaseous product (ethane, which is formed without any involvement of MMA, vide infra).

Table S 3. MMA polymerizations to form low molecular weight PMMA for end-group analysis.

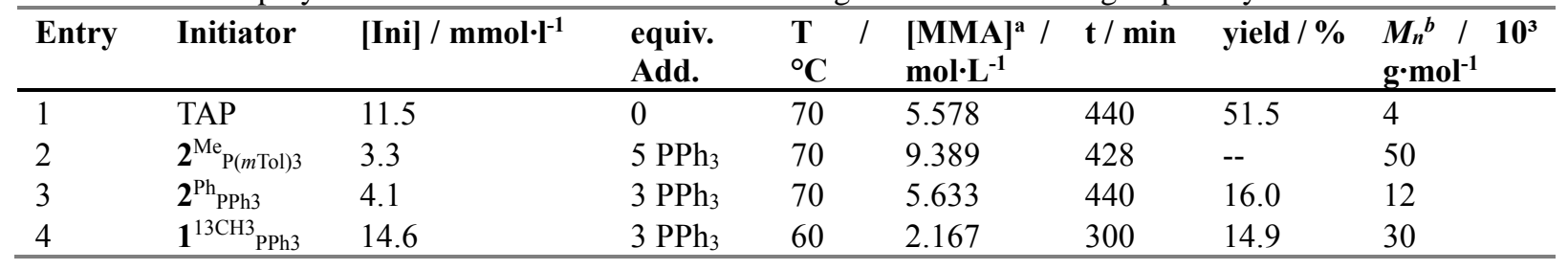

a If not bulk MMA $(9.389 \mathrm{M})$ toluene was used as solvent. b Determined by ${ }^{1} \mathrm{H}$ NMR $\left(400 \mathrm{MHz}, \mathrm{CD}_{2} \mathrm{Cl}_{2}, 25{ }^{\circ} \mathrm{C}\right)$ by integration of the unsaturated end-groups vs. the methoxy backbone resonances. 


\section{Reactivity of MMA toward Insertion Chain Growth Species}

Simultaneous polymerizations of ethylene and MMA were conducted at 25, 50 and 100 bar ethylene pressure. However, at high pressures ( $>25$ bar) a radical addition of ethylene to the growing radical chain becomes more feasible and statistical (co)polymers should form. ${ }^{28}$ Since we were interested in the interactions of pure ethylene insertion polymerization and a radical MMA polymerization, we majorly discuss the results obtained from polymerizations at 25 bar ethylene pressure. At 25 bar ethylene, $70{ }^{\circ} \mathrm{C}$, neat MMA with AIBN as a radical source a polymer with 99 mol-\% MMA content was obtained in the absence of any Ni(II) precursor.

General procedure for the simultaneous polymerization of ethylene in the presence of MMA at 25 bar ethylene pressure.

Polymerizations were conducted in a BÜCHI steel reactor with glass-inlet. The reactor was evacuated before polymerization for $1 \mathrm{~h}$ at $70{ }^{\circ} \mathrm{C}$ and was subsequently purged three times with argon inert gas. The glass inlet has shown to be crucial to prevent MMA self-initiation after removal of the stabilization agent (MEHQ) from the monomer solution. The desired amount of catalyst precursor and $\mathrm{PPh}_{3}$ was weighted in a Schlenk-tube, sealed with a rubber septum and removed from the glovebox, dissolved in $50 \mathrm{~mL}$ of cold MMA and transferred via cannula transfer into the preheated reactor and was stirred at $750 \mathrm{rpm}$. The reactor was pressurized to 25 bar ethylene and the ethylene valve was closed after saturation. After the desired time interval, the reactor was vented slowly and the resulting reaction mixture was poured into a large excess of $\mathrm{MeOH}$ to precipitate the polymer formed. The precipitate was filtered and dried under vacuum. The filtrate was reduced to dryness under reduced pressure.

General procedure for the simultaneous polymerization of ethylene in the presence of MMA at $>25$ bar ethylene.

Polymerizations were conducted in a $160 \mathrm{~mL}$ steel reactor from Parr Instrument equipped with safety valves, stirrer and oven. High-pressure ethylene was stored in a $1.5 \mathrm{~L}$ intermediate tank, by cooling the tank to $-20^{\circ} \mathrm{C}$ and liquefying ethylene at 35 bar. After reaching the thermodynamic equilibrium, the tank was isolated and heated to reach a pressure of approximately 200 bar. The desired amount of catalyst precursor and addition was dissolved in $50 \mathrm{~mL}$ of cold MMA and transferred into the reactor. The solution was stirred (250 rpm), heated to $70{ }^{\circ} \mathrm{C}$ and pressurized (50 or 100 bar ethylene). At the end of the reaction, the reactor was cooled and degassed. The polymer formed was isolated by evaporation of the volatile compounds and dried under vacuum at $70{ }^{\circ} \mathrm{C}$. 
Determination of the ethylene and MMA content in the polymers formed by simultaneous ethylene and MMA polymerization.

The molar fraction ( $x_{\mathrm{MMA}}$ ) of MMA in the polymer mixture formed after polymerization was estimated by ${ }^{1} \mathrm{H}$ NMR, setting the signal of the methoxy group (3.5 ppm) to a relative intensity of 3 vs. the integral of the aliphatic region (0.3-2.5 ppm, $I_{a l i p h}$, equation 2$){ }^{26 b}$

$$
x_{M M A}=\frac{1}{1+\frac{I_{\text {aliph }}-5}{4}}
$$

The corresponding weight-\% was calculated according to equation 3.

$$
w t \%(M M A)=\frac{x_{M M A} \cdot 100.12 \frac{g}{m o l}}{\left(x_{M M A} \cdot 100.12 \frac{g}{m o l}+\left(1-x_{M M A}\right) \cdot 28.05 \frac{g}{m o l}\right)} \cdot 100 \%
$$

The composition by mass was derived by equations 4 and 5 .

$$
\begin{aligned}
& m(P M M A)=m(\text { total }) \cdot\left(\frac{w t \%(M M A)}{100 \%}\right) \\
& m(P E)=m(\text { total }) \cdot\left(\frac{(1-w t \%(M M A))}{100 \%}\right)
\end{aligned}
$$

Here, $m$ (total) is the sum of the precipitated polymer and the filtrate corrected for added complex and additives. By this method, the soluble polymer fraction is taken into account.

Molecular weight determination of PE polymers in the polymer mixtures formed by simultaneous ethylene and MMA polymerization by ${ }^{1} H$ NMR.

For the calculation of the number average molecular weight of the PE fraction $\left(M_{\mathrm{n}, \mathrm{PE}, \mathrm{HNMR}}\right)$ it is assumed that the $\beta$-hydride elimination is the major chain termination mechanism. ${ }^{10}$ This allows to calculate the molecular weight with the intensity of the unsaturated chain-ends at 4.50-5.50 ppm (Figure S 23) vs. the PE backbone resonance. Due to the presence of PMMA the PE backbone signal is overlapped and the intensity of the aliphatic region has to be corrected. The signal intensity which is contributed from PMMA backbone resonances can be estimated by subtracting $5 / 3$ of the isolated methoxy resonance at 3.5 ppm (eq. 6). 


$$
M_{n P E, H N M R}=\frac{\left(\frac{I_{\text {aliph }}-\left(I_{O M e} \cdot \frac{5}{3}\right)}{4}\right)}{\left(\frac{I_{1}+I_{2}+2 I_{3}+2 I_{4}+I_{5}}{2}\right)} \cdot 28 \frac{g}{m o l}
$$

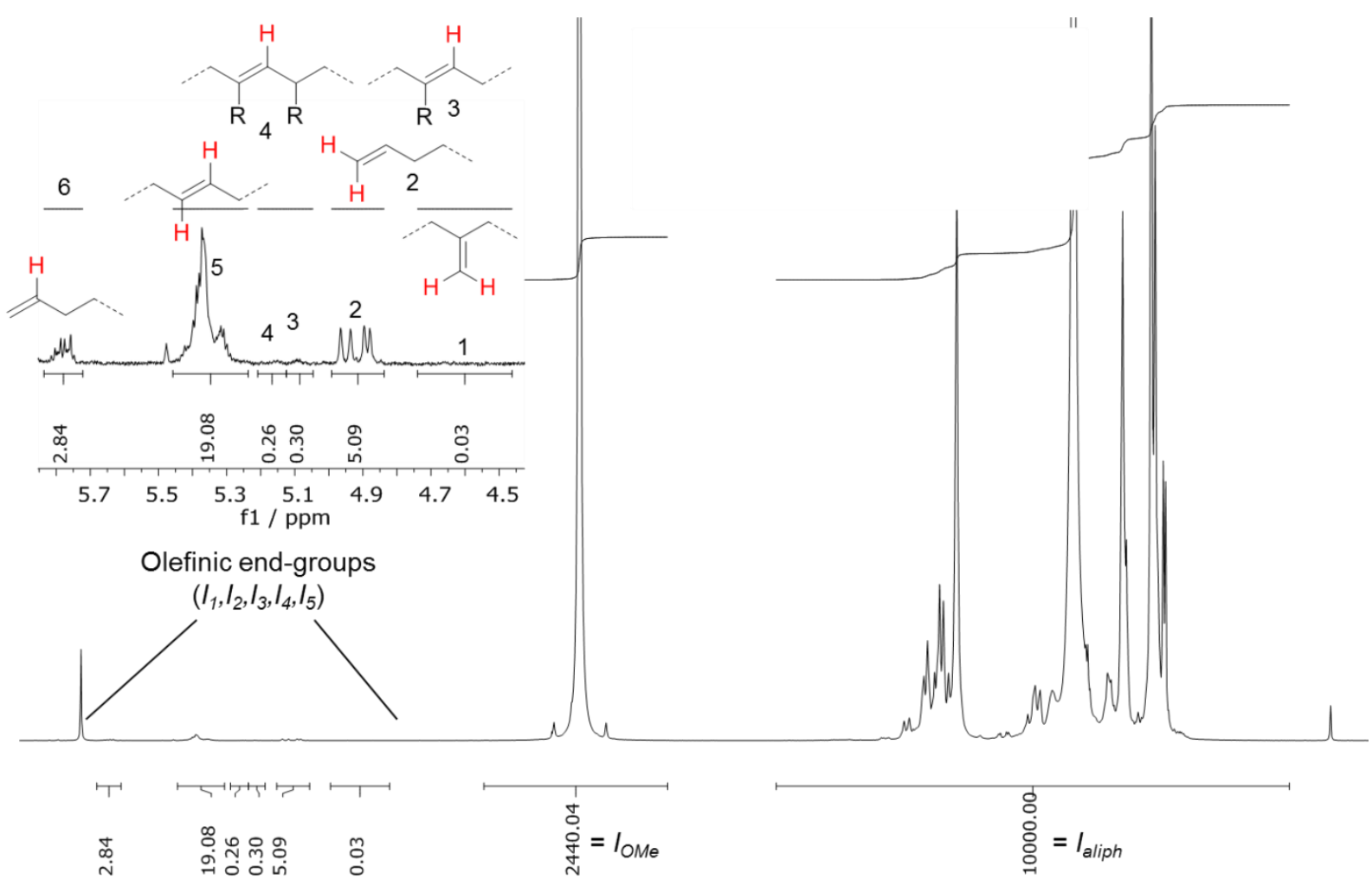

6.0 $5.85 .65 .45 .25 .04 .84 .64 .44 .24 .03 .83 .63 .43 .23 .02 .82 .62 .42 .22 .01 .8 \quad 1.6 \quad 1.4 \quad 1.2 \quad 1.0 \quad 0.8 \quad 0.6 \quad 0.4 \quad 0.2 \quad 0.0$ $\mathrm{f} 1 / \mathrm{ppm}$

Figure S 23. ${ }^{1} \mathrm{H}$ NMR spectrum $\left(600 \mathrm{MHz}\right.$, TCE- $\left.d_{2}, 130{ }^{\circ} \mathrm{C}\right)$ of a PE/PMMA polymer mixture obtained by reaction of $2^{13 \mathrm{CH}}{ }_{\mathrm{PPh} 3}, 3$ equiv. of $\mathrm{PPh}_{3}, 25$ bar of ethylene, $50 \mathrm{~mL}$ of MMA after $10 \mathrm{~min}$ polymerization time at $70{ }^{\circ} \mathrm{C}$. The integrals used for molecular weight determination of the PE fraction are given as inset.

Molecular weight determination of PE polymers in the polymers formed by simultaneous ethylene and MMA polymerization by ${ }^{13} C N M R$.

The uncorrected molecular weight $\left(M_{\mathrm{n}, \mathrm{PE}, \mathrm{UC}}\right)$ was determined from the signal intensity of the unsaturated methylene end-group signals $\left(\left(I_{\mathrm{S} 2}+I_{\mathrm{S} 3}\right) / 2=I_{\text {end-group, }}\right.$ Figure $\left.\mathrm{S} 28\right)$ in relation to the overall PE signal intensity $\left(I_{\text {tot }}\right)$ and multiplied by $14.03 \mathrm{~g} \cdot \mathrm{mol}^{-1}$ (equation 7 ).

$$
M_{n, P E, U C}=\frac{I_{\text {tot }}}{I_{\text {end-group }}} \cdot 14.03 \frac{\mathrm{g}}{\mathrm{mol}}
$$

Due to „chain walking“ the formation of internal double bonds was observed (about $80 \%$ ) and has to be included into the calculation, because polymer chains with internal double bonds contain two saturated end-groups. Herein, it is assumed that these double bonds are remote to 
the S2 and S3 groups, thus the typical resonances for the S2 and S3 groups are observed. Not in all cases signals of the unsaturated chain-ends were detected in the ${ }^{13} \mathrm{C} N M R$ spectrum, therefore the fraction of internal versus terminal double bonds was determined by ${ }^{1} \mathrm{H}$ NMR. The corrected signal intensity for the saturated end-groups is calculated as follows (equation $8)$ :

$$
I_{S 2, S 3, \text { corr }}=\left(x_{\text {unsat.terminal }} \cdot \frac{\left(I_{S 2}+I_{S 3}\right)}{2}\right)+(1-x) \cdot\left(\frac{\left(I_{S 2}+I_{S 3}\right)}{4}\right)
$$

By initiation of the ethylene insertion polymerization with [Ni-Me] complex precursors the corrected molecular weights $\left(M_{\mathrm{n}, \mathrm{PE}, \mathrm{CNMR}}\right)$ were calculated according to:

$$
M_{n, P E, C N M R}=\frac{I_{\text {tot }}}{I_{\text {end-group }}} \cdot 14.03 \frac{\mathrm{g}}{\mathrm{mol}}=\frac{I_{\text {tot }}}{I_{S 2, S 3, \text { corr }}} \cdot 14.03 \mathrm{~g} \cdot \mathrm{mol}^{-1}
$$

By initiation with [Ni-Ph] catalyst precursors additional phenyl end-groups are present (Figure $\mathrm{S}$ 24). The fraction of phenyl end-groups vs. unsaturated end-groups was determined by ${ }^{1} \mathrm{H}$ NMR by integration of the aromatic resonance at 7.00-7.25 ppm $\left(I_{\mathrm{Ph}}\right)$ and the olefinic resonances at 4.50-5.50 ppm (equation 10).

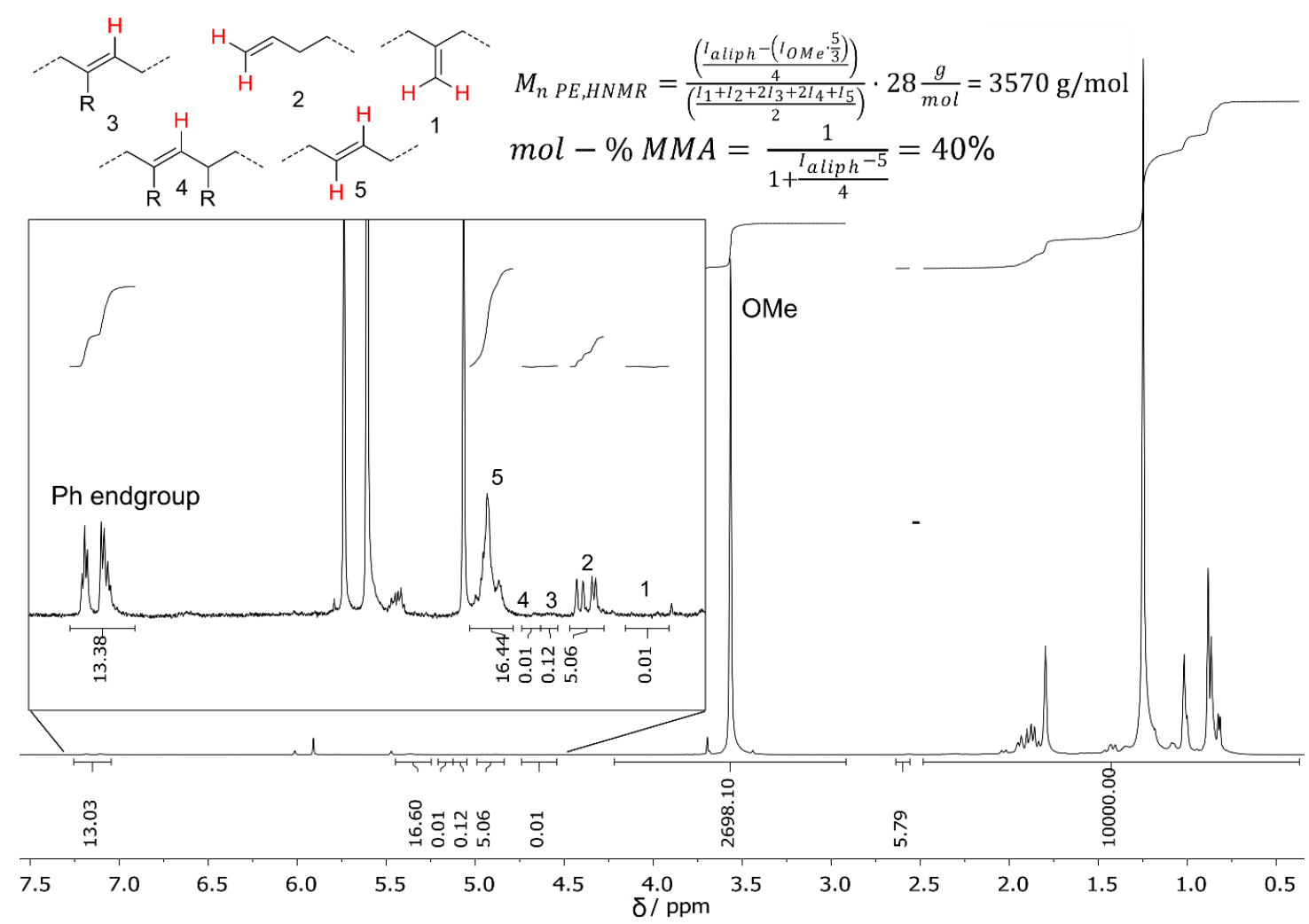

Figure S 24. ${ }^{1} \mathrm{H}$ NMR spectrum $\left(600 \mathrm{MHz}\right.$, TCE- $d_{2}, 130{ }^{\circ} \mathrm{C}, 30$ s relaxation delay, 16 scans $)$ of a PE/PMMA polymer mixture obtained by reaction of $2^{\mathrm{Ph}}{ }_{\mathrm{PPh} 3}, 3$ equiv. of $\mathrm{PPh}_{3}, 25$ bar of ethylene, $50 \mathrm{~mL}$ of MMA after $5 \mathrm{~min}$ polymerization time at $70{ }^{\circ} \mathrm{C}$. The inset is showing the assignment of the unsaturated end-groups and the calculation of the molecular weight derived from ${ }^{1} \mathrm{H}$ NMR for the PE fraction $\left(M_{\mathrm{n}, \mathrm{PE}, \mathrm{HNMR}}\right)$. 


$$
x_{P h}=\frac{\left(\frac{I_{P h}}{5}\right)}{\left(\frac{I_{1}+I_{2}+2 I_{3}+2 I_{4}+I_{5}}{2}\right)}
$$

The internal double bond and phenyl end-group corrected signal intensity of the S2 and S3 endgroups ( $\left.I_{\text {end-group }}=I_{S 2, S 3, \text { Phcorr }}\right)$ was calculated according to equation 11 and was used to calculate $M_{\mathrm{n}, \mathrm{PE}, \mathrm{CNMR}}$ applying equation 7.

$$
I_{\text {end-group }}=I_{S 2, S 3, \text { corr }}+\left(x_{P h} \cdot\left(\frac{I_{S 2, S 3, \text { corr }}}{\left(1-\left(x_{P h}\right)\right)}\right)\right)
$$

\subsection{Studies on the migratory insertion initiation process using labeled $\left[\mathrm{Ni}^{13} \mathrm{CH}_{3}\right]$ and $[\mathrm{Ni}-\mathrm{Ph}]$ complexes.}

Calculation of the initiation efficiency via ${ }^{13} \mathrm{C}$ labeling experiments.

The percentage of ${ }^{13} \mathrm{C}$ label in the PE polymer formed was derived by comparison of $I_{\mathrm{S} 1}$ and $I_{\mathrm{S} 2}$. Both were corrected for additional intensity resulting from "chain walking".

$$
I_{S 2, \text { corr }}=\left(x_{\text {terminal }} \cdot I_{S 2}\right)+\left(\left(1-x_{\text {terminal }}\right) \cdot\left(\frac{I_{S 2}}{2}\right)\right)
$$

The corrected $I_{\mathrm{S} 1 \text {, corr }}$ was obtained by subtracting the intensity of the $I_{\mathrm{S} 2}$ signal which is introduced by "chain walking".

$$
I_{S 1, \text { corr }}=I_{S 1}-I_{S 2, C W}
$$

By comparing the unlabeled $I_{\mathrm{S} 2 \text {,corr }}$ with the intensity of the partly ${ }^{13} \mathrm{C}$-labeled methyl endgroups $I_{\mathrm{S} 1 \text {,corr, }}$ the fraction of labeled polymer chains was obtained (equation 14).

$$
x_{13 C}=\frac{\left(\frac{I_{S 1, \text { corr }}}{I_{S 2, \text { corr }}}-1\right)}{89.909091}
$$

The total amount of PE chains was calculated from the total yield of the polymerization divided by the molecular weight obtained via ${ }^{1} \mathrm{H}$ or ${ }^{13} \mathrm{C} \mathrm{NMR}$, and the amount of labeled polymer chains $\left(\mathrm{n}\left({ }^{13} \mathrm{C}-\mathrm{PE}\right)\right)$ was obtained by multiplying this value with the fraction of labeled chains (equation $15)$. 


$$
n\left({ }^{13} C-P E\right)=x_{13 C} \cdot \frac{m_{\text {total }}}{M_{P E, N M R}}
$$

Finally, the percentage of catalyst precursor which has initiated a PE chain $\left(x_{\text {ini }}\right)$ was derived by dividing the amount of labeled chains by the amount of supplied catalyst precursor $(\mathrm{n}([\mathrm{Ni}])$, equation 16).

$$
x_{i n i}=\frac{n\left({ }^{13} C-P E\right)}{n([N i])}
$$

With the fraction of the catalyst precursor which was activated by ethylene, the amount of chain transfers can be calculated according to equation 17 .

$$
Z_{C T}=\left(\frac{n(P E)}{n([N i]) \cdot x_{i n i}}\right)-1
$$

\begin{tabular}{|c|c|c|c|c|c|c|c|c|c|c|}
\hline & Complex & $\begin{array}{l}\mathrm{n}([\mathrm{Ni}]) / \\
\text { mmol }\end{array}$ & $\begin{array}{l}\mathbf{t} \\
/ \\
\min \end{array}$ & $\begin{array}{l}\text { yield } \\
\text { a / g }\end{array}$ & $\begin{array}{l}\text { mol-\% } \\
\text { MMA }^{b}\end{array}$ & $\begin{array}{l}\mathbf{M}_{\mathrm{n}} / \mathbf{1 0}^{\mathbf{3}} \\
\mathrm{g} \cdot \mathrm{mol}^{-1} \\
{ }^{1} \mathbf{H} \mid{ }^{13} \mathrm{C}\end{array}$ & $\begin{array}{l}\text { Is1 } \\
\text { a.u. }\end{array}$ & $\begin{array}{l}\text { Is2 / } \\
\text { a.u. }\end{array}$ & $\begin{array}{l}\% \text {-ini } \\
{ }^{1} \mathrm{H} \mid{ }^{13} \mathrm{C}\end{array}$ & $\begin{array}{l}\mathbf{Z}_{\mathrm{CT}} \\
{ }^{1} \mathbf{H} \mid{ }^{13} \mathrm{C}\end{array}$ \\
\hline 1 & $\mathbf{1}^{13 \mathrm{CH} 3}{ }_{\mathrm{PPh} 3}$ & 15.1 & 5 & 0.40 & 55 & $3.2 \mid 2.2$ & 112.0 & 15.9 & $17 \mid 26$ & $8 \mid 7$ \\
\hline 2 & $\mathbf{1}^{13 \mathrm{CH} 3}{ }_{\mathrm{PPh} 3}$ & 14.7 & 5 & 0.46 & 79 & $2.1 \mid 3.3$ & 188.9 & 11.3 & $28 \mid 18$ & $2 \mid 3$ \\
\hline 3 & $\mathbf{2}^{13 \mathrm{CH} 3} \mathrm{PPh} 3$ & 16.0 & 10 & 0.56 & 36 & $3.3 \mid 2.5$ & 98.6 & 14.5 & $37 \mid 49$ & $8 \mid 8$ \\
\hline 4 & $\mathbf{2}^{13 \mathrm{CH} 3} \mathrm{PPh} 3$ & 14.8 & 10 & 2.99 & -- & $3.7 \mid 4.1$ & 15.2 & 7.6 & $96 \mid 89$ & $54 \mid 54$ \\
\hline
\end{tabular}

The results are summarized in Table S 4.

Table S 4. Results of the calculation of the initiation efficiency for [Ni-Me] complexes.

Reaction conditions: 3 equiv. of $\mathrm{PPh}_{3}, \mathrm{~T}=70^{\circ} \mathrm{C}$, $\mathrm{p}$ (ethylene) $=25$ bar, $\mathrm{V}(\mathrm{MMA})=50 \mathrm{~mL}$. a Sum of precipitate and reduced filtrate minus $\mathrm{m}_{\mathrm{Ni}}$ and $\mathrm{m}_{\text {add }}$. b Determined by ${ }^{1} \mathrm{H}-\mathrm{NMR}$ spectroscopy.

As standard conditions for the acquisition of ${ }^{13} \mathrm{C}$ NMR spectra an inverse gated decoupling sequence with a relaxation delay of $2.0 \mathrm{~s}$ and an acquisition time of $1.0 \mathrm{~s}$ and about 12000 repeated scans were used. To all samples $1 \mathrm{mg} \mathrm{Cr}(\mathrm{AcAc})$ per $\mathrm{mL} \mathrm{TCE}-d_{2}$ was added as relaxation aid. We were using these relative short acquisition times because an experiment with $4.0 \mathrm{~s}$ relaxation delay, $3.1 \mathrm{~s}$ acquisition time, 50000 scans and $5 \mathrm{mg} \mathrm{Cr}(\mathrm{AcAc})$ per $\mathrm{mL}$ TCE- $d_{2}$ led to similar values for the initiation efficiency. For sample Table S 4 Entry 3 the more accurate acquisition method (see Figure S 25) resulted in a $M_{\mathrm{n}, \mathrm{PE}, \mathrm{CNMR}}$ of $3000 \mathrm{~g} \cdot \mathrm{mol}^{-1}$ and \%-initiation efficiency of $45 \%$ and $46 \%$ for ${ }^{1} \mathrm{H}$ and ${ }^{13} \mathrm{C}$ derived values, respectively. However, a multiplication of the needed spectrometer time by a factor more than 10 (10 h vs. 5 days for one sample) comes along with the more accurate method. 


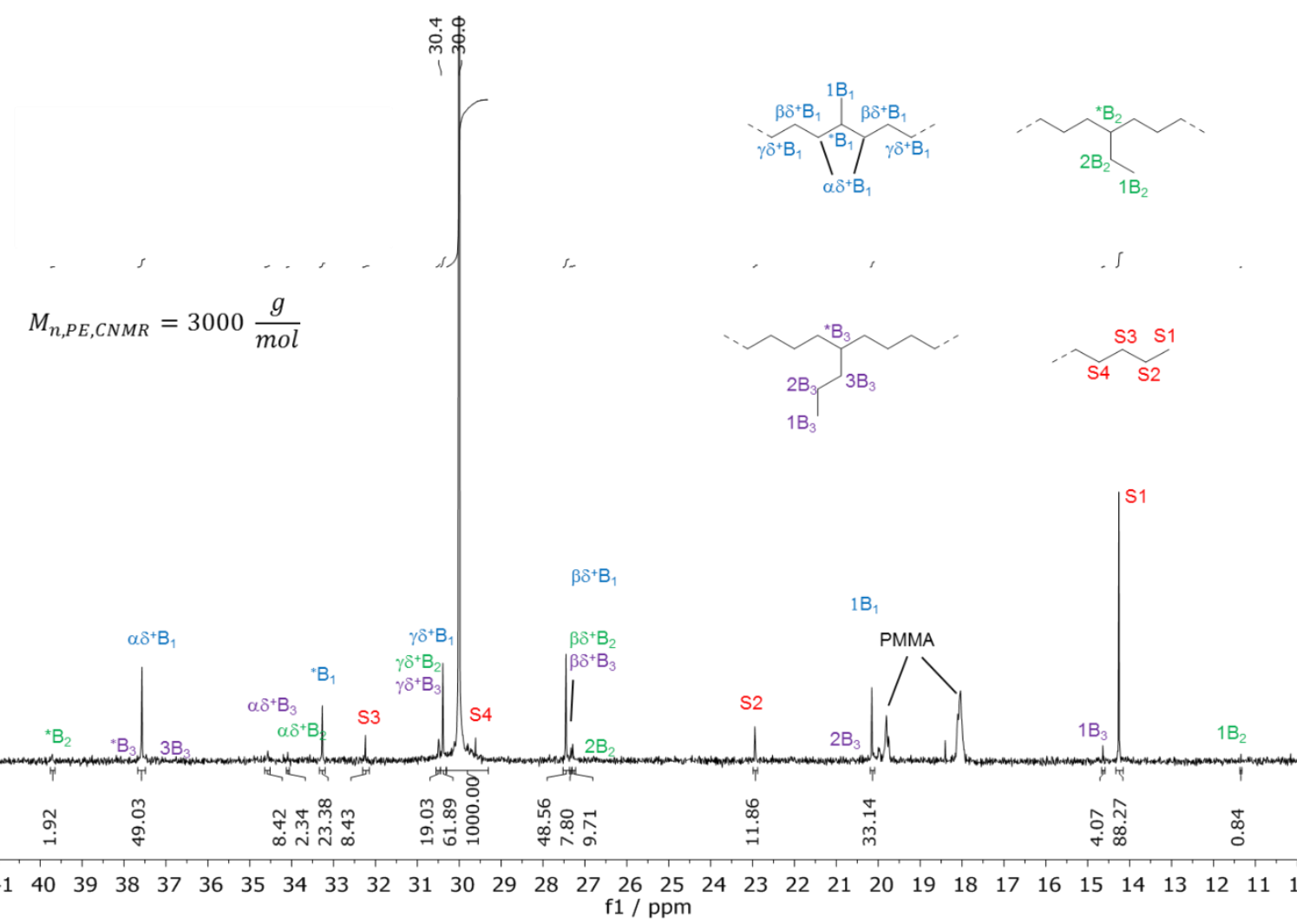

Figure S 25. Aliphatic region of the ${ }^{13} \mathrm{C}\left\{{ }^{1} \mathrm{H}\right\}$ NMR spectrum $\left(151 \mathrm{MHz}\right.$, TCE- $d_{2}, 130{ }^{\circ} \mathrm{C}, 4 \mathrm{~s}$ relaxation delay, $3.1 \mathrm{~s}$ acquisition time, 50000 scans, inverse gated decoupling) of a PE/PMMA polymer mixture obtained by reaction of $2^{13 \mathrm{CH} 3}{ }_{\mathrm{PPh} 3}, 3$ equiv. of $\mathrm{PPh}_{3}, 25$ bar of ethylene, $50 \mathrm{~mL}$ of MMA after 10 min polymerization time at $70{ }^{\circ} \mathrm{C}$.

\section{Calculation of the initiation efficiency via phenyl incorporation into the PE polymer.}

It is assumed that the major termination reaction of the migratory insertion polymerization of ethylene is the $\beta$-hydride elimination and that all chains contain one unsaturated end-group and chains which were initiated by the supplied $[\mathrm{Ni}-\mathrm{Ph}]$ complex contain one phenyl group. The ratio between the phenyl- and double bond end-groups was determined by ${ }^{1} \mathrm{H}$ NMR (see Figure $\mathrm{S} 24$, eq. (10)). The amount of phenyl terminated PE chains (n(Ph-PE)), the fraction of activated catalyst $\left(x_{\text {ini }}\right)$ and the chain transfer number $\left(Z_{\mathrm{CT}}\right)$ was calculated in analogy to the ${ }^{13} \mathrm{C}$ labeling experiments (vide supra, results Table S 5).

Table S 5. Results of the calculation of the initiation efficiency for [Ni-Ph] complexes.

\begin{tabular}{|c|c|c|c|c|c|c|c|c|c|c|}
\hline & Complex & $\begin{array}{l}\mathrm{N}([\mathrm{Ni}]) / \\
\text { mmol }\end{array}$ & $\begin{array}{l}\mathbf{t} \\
/ \\
\min \end{array}$ & $\begin{array}{l}\text { yield }^{\mathrm{a}} \\
/ \mathrm{g}\end{array}$ & $\begin{array}{l}\text { mol-\% } \\
\text { MMA }^{\mathbf{b}}\end{array}$ & $\begin{array}{l}M_{\mathrm{n}} / \mathbf{1 0}^{3} \\
\mathrm{~g} \cdot \mathrm{mol}^{-1} \\
\left.{ }^{1} \mathrm{H}\right|^{13} \mathrm{C}\end{array}$ & $\begin{array}{l}\text { IPh / } \\
\text { a.u. }\end{array}$ & $\begin{array}{l}\text { Iunsat } \\
\text { / a.u. }\end{array}$ & $\begin{array}{l}\% \text {-ini. } \\
{ }^{1} \mathrm{H} \mid{ }^{13} \mathrm{C}\end{array}$ & $\begin{array}{l}Z_{\mathrm{CT}} \\
{ }^{1} \mathrm{H} \mid{ }^{13} \mathrm{C}\end{array}$ \\
\hline 1 & $2^{\mathrm{Ph}}{ }_{\mathrm{PPh} 3}$ & 16.1 & 5 & 0.409 & 40 & $3.6 \mid 3.6$ & 13.4 & 21.8 & $52 \mid 52$ & $3 \mid 3$ \\
\hline 2 & $\mathbf{2}^{\mathrm{Ph}}{ }_{\mathrm{PPh} 3}$ & 14.8 & 10 & 0.488 & 56 & $2.8 \mid 3.2$ & 11.4 & 19.4 & $50 \mid 44$ & $3 \mid 3$ \\
\hline 3 & $\mathbf{2}^{\mathrm{Ph}}{ }_{\mathrm{PPh} 3}$ & 19.7 & 30 & 0.680 & 51 & $3.3 \mid 3.0$ & 10.8 & 18.0 & $54 \mid 59$ & $3 \mid 3$ \\
\hline 4 & $2^{\mathrm{Ph}} \mathrm{PPh}_{3}$ & 14.5 & 120 & 0.595 & 46 & $3.0 \mid 3.2$ & 10.5 & 22.9 & $62 \mid 58$ & $4 \mid 4$ \\
\hline
\end{tabular}

Reaction conditions: 3 equiv. $\mathrm{PPh}_{3}, \mathrm{~T}=70^{\circ} \mathrm{C}$, $\mathrm{p}$ (ethylene) $=25$ bar, $\mathrm{V}(\mathrm{MMA})=50 \mathrm{~mL}$. a Sum of precipitate and reduced filtrate minus $\mathrm{m}_{\mathrm{Ni}}$ and $\mathrm{m}_{\mathrm{add}}$. b Determined by ${ }^{1} \mathrm{H}-\mathrm{NMR}$ spectroscopy. 
Further discussion of the initiation efficiency of the ethylene migratory insertion polymerization.

The insertion and the free radical polymerization was shown to be active on the same timescale and the generation of free radical polymeryl chains is faster in the presence of ethylene. Here, a deeper knowledge of the role of the catalyst precursor under the conditions applied is required. To this end, labeled $\left[\mathrm{Ni}_{-}{ }^{13} \mathrm{CH}_{3}\right]\left(2^{13 \mathrm{CH} 3}{ }_{\mathrm{PPh} 3}\right)$ and $[\mathrm{Ni}-\mathrm{Ph}]\left(\mathbf{2}^{\mathrm{Ph}}{ }_{\mathrm{PPh} 3}\right)$ catalyst precursors were used, which introduce a ${ }^{13} \mathrm{CH}_{3}$ label or a phenyl group to the PE chain end and therefore allow for a quantification (NMR spectroscopy) of the amount of catalyst precursor initiating a ethylene insertion chain growth polymerization (\%-ini., Table S 6).

After 5 minutes of reaction time, approximately $50 \%$ of $2^{\mathrm{Ph}}{ }_{\mathrm{PPh} 3}$ was already activated by ethylene, and the extent of activation eventually further increased up to $62 \%$ after 120 minutes (Table S 6, Entries 1-4). At the same time, the overall MMA content of the polymer composition formed was $40 \%$ (mol-\% MMA-based repeat units) after $5 \mathrm{~min}$, increased to $56 \%$ after 10 minutes and finally decreased to $46 \%$ after 120 minutes. This implies that the free radical polymerization is mainly active during the reaction period, when a high concentration of active species of the ethylene polymerization is present in the reaction mixture (up to $30 \mathrm{~min}$ ).

Table S 6. Initiation efficiency (\%-ini) for $[\mathrm{Ni}-\mathrm{Ph}]$ and $\left[\mathrm{Ni}^{-13} \mathrm{CH}_{3}\right]$ complexes in the polymerization of ethylene w/ or w/o MMA.

\begin{tabular}{lllll}
\hline Entry & Complex & t / min & $\begin{array}{l}\text { MMA content in } \begin{array}{l}\text { o-ini for } \\
\text { polym. formed }\end{array} \\
\text { polym. }\end{array}$ \\
\hline $\mathbf{1}^{\mathbf{b}}$ & $\mathbf{2}^{\mathrm{Ph}}{ }_{\mathrm{PPh} 3}$ & 5 & 40 & 52 \\
$\mathbf{2}^{\mathbf{b}}$ & $\mathbf{2}^{\mathrm{Ph}}{ }_{\mathrm{PPh} 3}$ & 10 & 56 & 50 \\
$\mathbf{3}^{\mathbf{b}}$ & $\mathbf{2}^{\mathrm{Ph}} \mathrm{PPh}^{\mathrm{b}}$ & 30 & 51 & 54 \\
$\mathbf{4}^{\mathbf{b}}$ & $\mathbf{2}^{\mathrm{Ph}}{ }_{\mathrm{PPh} 3}$ & 120 & 46 & 62 \\
$\mathbf{5}^{\mathbf{b}}$ & $\mathbf{2}^{13 \mathrm{CH} 3} \mathrm{PPh} 3$ & 10 & 36 & 49 \\
$\mathbf{6}^{\mathbf{c}}$ & $\mathbf{2}^{13 \mathrm{CH} 3}{ }_{\mathrm{PPh} 3}$ & 10 & 0 & 89 \\
\hline
\end{tabular}

Reaction conditions: $70{ }^{\circ} \mathrm{C}, \mathrm{p}(\mathrm{E})=25$ bar, 3 equiv. of $\mathrm{PPh}_{3}$. For details and calculation see SI. (a) Determined by ${ }^{1} \mathrm{H}$ NMR spectroscopy. Given as mol-\% MMA-based repeat units. (b) MMA was used as a solvent. (c) Toluene was used as a solvent.

Afterwards, the mol-\% MMA slightly drops and the E polymerization slightly predominates over the radical MMA polymerization (mol-\% E increased in the product mixture). Both catalyst precursors $2^{13 \mathrm{CH} 3}{ }_{\mathrm{PPh} 3}$ and $2^{\mathrm{Ph}}{ }_{\mathrm{PPh} 3}$ (Table S 6, Entries 2 vs. 5) were activated by ethylene to ca. $50 \%$ after $10 \mathrm{~min}$, therefore, the activation rate by ethylene seems to be similar for the [Ni-Me] and [Ni-Ph] complex here. In toluene, after 10 minutes $89 \%$ of $2^{13 \mathrm{CH}^{3}}{ }_{\mathrm{PPh} 3}$ was activated by ethylene (Table S 6, Entry 6). This indicates that in analogy to the reduced ethylene productivity in the presence of MMA (vide supra), the initiation of the precursor complexes by ethylene is significantly slowed down in the presence of MMA, which again can be accounted 
for by the coordination of added $\mathrm{PPh}_{3}$ and the coordination of the ester group of MMA. Although the insertion polymerization of ethylene is slowed down in the presence of MMA, the free radical MMA polymerization is accelerated in the presence of ethylene 
Table S 7. Simultaneous ethylene and MMA polymerizations mediated by $\mathbf{1}^{\mathrm{Me}}{ }_{\mathrm{PPh} 3}, \mathbf{2}^{\mathrm{Me}}{ }_{\mathrm{PPh} 3}, \mathbf{2}^{\mathrm{Ph}}{ }_{\mathrm{PPh} 3}$ and ${ }^{13} \mathrm{C}$ labeled [Ni-Me] catalyst precursors.

\begin{tabular}{|c|c|c|c|c|c|c|c|c|c|c|c|c|c|c|c|}
\hline $\begin{array}{l}\text { Ent } \\
\text { ry }\end{array}$ & $\begin{array}{l}\text { Comple } \\
\mathrm{x}\end{array}$ & $\begin{array}{l}\mathrm{n}([\mathrm{Ni}]) / \\
\mu \mathrm{mol}\end{array}$ & $\begin{array}{l}\mathrm{t} \\
/ \\
\mathrm{mi} \\
\mathrm{n}\end{array}$ & $\begin{array}{l}\text { yield }^{\mathrm{a}} \\
/ \mathrm{g}\end{array}$ & $\begin{array}{l}\text { Productivi } \\
\text { ty } \mathrm{wg}^{\mathrm{b}} \mathrm{mg}^{*} \\
\mathrm{mmol}^{-1}\end{array}$ & $\begin{array}{l}\text { mol- } \% \\
\text { MMA }^{\mathrm{c}}\end{array}$ & $\begin{array}{l}\mathrm{T}_{\mathrm{m}} \mid \mathrm{T}_{\mathrm{g}}^{\mathrm{d}} \\
/{ }^{\circ} \mathrm{C}\end{array}$ & $\begin{array}{l}\text { Cryst. } \\
/ \%\end{array}$ & $\begin{array}{l}N_{\text {unsa }} \\
/ N_{\text {act }}{ }^{`}\end{array}$ & $\begin{array}{l}\mathrm{t}_{\mathrm{n}}(\mathrm{PE})^{\mathrm{f}} \\
\mathrm{g} \cdot \mathrm{mol}^{-1} \\
{ }^{1} \mathrm{H} \mid{ }^{13} \mathrm{C}\end{array}$ & $0^{30} \% \mathrm{rr}^{\mathrm{g}}$ & $\begin{array}{l}\text { Branches }^{\mathrm{h}} \\
/ 1000 \mathrm{C} \\
\mathrm{Me}|\mathrm{Et}| \operatorname{Pr}\end{array}$ & $\begin{array}{l}\mathrm{M}_{\mathrm{n}}(\mathrm{SEC} \\
/ 10^{3} \mathrm{~g} \cdot \mathrm{m} \\
1\left(\bigoplus_{M}\right)\end{array}$ & $\begin{array}{l}\text {, } 40)^{\mathrm{i}} \\
2\left(\bigoplus_{M}\right)\end{array}$ & $\begin{array}{l}\mathrm{M}_{\mathrm{n}}(\mathrm{SEC}, 160)^{\mathrm{j}} \\
/ 10^{3} \mathrm{~g} \cdot \mathrm{mol}^{-1}\left(\bigoplus_{M}\right)\end{array}$ \\
\hline 1 & $\mathbf{1}_{\mathrm{PPh} 3}^{\mathrm{Me}}$ & 15.4 & 5 & 0.554 & 36.1 & 73 & 108 | 123 & 10.9 & 1.0 & $4.6 \mid 4.7$ & 63 & $17|3| 3$ & $4.4(1.3)$ & $108.6(2.2)$ & \\
\hline 2 & $\mathbf{1}^{\mathrm{Me}}{ }_{\mathrm{PPh} 3}$ & 14.9 & 10 & 0.655 & 44.0 & 55 & $108 \mid 123$ & 12.0 & 0.7 & $4.5 \mid 6.2$ & 64 & $17|2| 2$ & $4.6(1.3)$ & $92.9(2.5)$ & \\
\hline 3 & $\mathbf{1}^{\mathrm{Me}}{ }_{\mathrm{PPh} 3}$ & 16.7 & 30 & 0.966 & 57.7 & 52 & $107 \mid 122$ & 8.9 & 0.9 & $6.1 \mid 7.1$ & 63 & $17|2| 3$ & $4.5(1.3)$ & $114.4(2.6)$ & \\
\hline 4 & $\mathbf{1}^{\mathrm{Me}}{ }_{\mathrm{PPh} 3}$ & 15.1 & 60 & 0.867 & 57.3 & 48 & 125 & 10.4 & 0.8 & $6.2 \mid 7.4$ & 64 & $17|2| 2$ & $4.5(1.3)$ & $103.1(2.6)$ & \\
\hline 5 & $\mathbf{1}^{\mathrm{Me}}{ }_{\mathrm{PPh} 3}$ & 15.3 & 120 & 0.889 & 58.1 & 58 & $106 \mid 123$ & 6.9 & n.d. & n.d.|n.d. & n.d. & n.d. & $5.6(1.1)$ & $108.6(2.2)$ & \\
\hline 6 & $\mathbf{2}^{\mathrm{Me}}{ }_{\mathrm{PPh} 3}$ & 17.3 & 5 & 0.515 & 29.8 & 51 & $86 \mid 124$ & 17.9 & 0.7 & $2.5 \mid 3.6$ & 64 & $22|2| 3$ & $5.6(1.4)$ & 68.4 (1.7) & \\
\hline 7 & $2^{\mathrm{Me}}{ }_{\mathrm{PPh} 3}$ & 14.9 & 10 & 0.390 & 26.2 & 54 & $84 \mid 124$ & 13.6 & 0.7 & $2.7 \mid 4.0$ & 65 & $22|2| 4$ & $5.6(1.4)$ & $73.1(3.9)$ & $2.2(2.2)$ \\
\hline 8 & $2^{\mathrm{Me}}{ }_{\mathrm{PPh} 3}$ & 14.3 & 30 & 0.445 & 31.1 & 50 & $81 \mid 126$ & 11.8 & 0.9 & $2.6 \mid 3.0$ & 65 & $22|2| 4$ & $5.0(1.3)$ & $102.1(5.3)$ & \\
\hline 9 & $2^{\mathrm{Me}}{ }_{\mathrm{PPh} 3}$ & 15.3 & 60 & 0.634 & 41.4 & 47 & $83 \mid 124$ & 13.6 & 0.9 & $3.0 \mid 3.4$ & 63 & $26|3| 4$ & $5.5(1.3)$ & $69.3(2.2)$ & \\
\hline 10 & $2^{\mathrm{Me}}{ }_{\mathrm{PPh} 3}$ & 16.6 & 120 & 0.713 & 43.0 & 43 & $86 \mid 124$ & 13.9 & 1.2 & $4.4 \mid 3.6$ & 62 & $20|2| 4$ & $6.0(1.3)$ & $99.0(2.3)$ & \\
\hline 11 & $2^{\mathrm{Ph}}{ }_{\mathrm{PPh} 3}$ & 16.1 & 5 & 0.409 & 25.4 & 40 & $81 \mid 123$ & 15.4 & 1.0 & $3.6 \mid 3.6$ & 64 & $23|2| 3$ & $3.8(1.5)$ & $48.3(2.1)$ & \\
\hline 12 & $2^{\mathrm{Ph}}{ }_{\mathrm{PPh}}$ & 14.8 & 10 & 0.488 & 32.9 & 56 & $86 \mid 123$ & 10.8 & 0.9 & $2.8 \mid 3.2$ & 65 & $22|2| 3$ & $4.6(1.3)$ & $58.7(1.8)$ & \\
\hline 13 & $2^{\mathrm{Ph}}{ }_{\mathrm{PPh}}$ & 19.7 & 30 & 0.680 & 34.6 & 51 & $86 \mid 124$ & 12.0 & 1.1 & $3.3 \mid 3.0$ & 64 & $23|2| 3$ & $4.7(1.3)$ & $60.3(2.4)$ & \\
\hline 14 & $2^{\mathrm{Ph}}{ }_{\mathrm{PPh} 3}$ & 15.2 & 60 & 0.559 & 36.9 & 42 & $87 \mid 124$ & 12.6 & 1.0 & $3.6 \mid 3.5$ & 62 & $22|2| 3$ & $4.6(1.4)$ & 72.8 (3.3) & \\
\hline 15 & $2^{\mathrm{Ph}}{ }_{\mathrm{PPh}}$ & 14.5 & 120 & 0.595 & 41.0 & 46 & $85 \mid 124$ & 10.5 & 0.9 & $3.0 \mid 3.2$ & 64 & $23|3| 4$ & $6.3(1.3)$ & $84.0(3.7)$ & \\
\hline 16 & $\mathbf{1}^{13 \mathrm{CH} 3}{ }_{\mathrm{PPh} 3}$ & 15.1 & 5 & 0.40 & 26.5 & 55 & n.d. |n.d. & n.d. & 1.5 & $3.2 \mid 2.2$ & 63 & $18|0| 4$ & $5.5(1.2)$ & $103.1(2.2)$ & \\
\hline 17 & $\mathbf{1}^{13 \mathrm{CH}_{3}{ }_{\mathrm{PPh}} 3}$ & 14.7 & 5 & 0.46 & 31.2 & 79 & $107 \mid 126$ & 12.7 & 0.7 & $2.1 \mid 3.3$ & 65 & $18|0| 0$ & $4.2(1.2)$ & 86.8 (1.8) & \\
\hline 18 & $2^{13 \mathrm{CH}^{3}}{ }_{\mathrm{PPh} 3}$ & 16.0 & 10 & 0.56 & 35.0 & 36 & $97 \mid 125$ & 14.5 & 1.3 & $3.3 \mid 2.5$ & 64 & $25|3| 4$ & $6.3(1.2)$ & $64.2(2.4)$ & \\
\hline $19^{\mathrm{k}}$ & $2^{13 \mathrm{CH}^{3}}{ }_{\mathrm{PPh} 3}$ & 14.8 & 10 & 2.99 & 202.4 & 0 & $105 \mid--$ & 50.3 & 0.9 & $3.7 \mid 4.1$ & -- & $19|2| 4$ & $4.8(1.3)$ & not present & $2.9(2.1)$ \\
\hline
\end{tabular}

Reaction conditions: $\mathrm{T}=70^{\circ} \mathrm{C}, \mathrm{p}$ (ethylene) $=25 \mathrm{bar}, \mathrm{V}(\mathrm{MMA})=50 \mathrm{~mL}, 3$ equiv. $\mathrm{PPh}_{3}$. a Sum of precipitated polymer and the reduced filtrate with subtraction of catalyst precursor and PPh 3 . $\mathbf{b}$

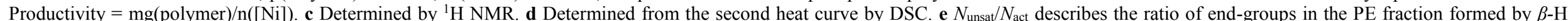

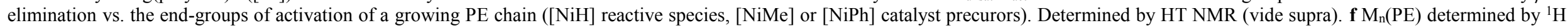

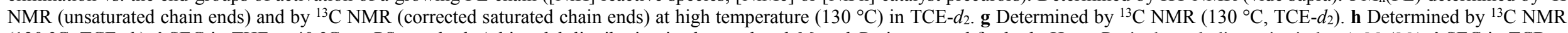
$\left(130{ }^{\circ} \mathrm{C}\right.$, TCE- $\left.d_{2}\right)$. $\mathbf{i}$ SEC in THF at $40{ }^{\circ} \mathrm{C}$ vs. PS standard. A bimodal distribution is observed and $M_{\mathrm{n}}$ and $\bigoplus_{M}$ is reported for both. Here, $\bigoplus_{M}$ is the polydispersity index $\left(=M_{\mathrm{w}} / M_{\mathrm{n}}\right) \mathbf{j}$ SEC in TCB at $160{ }^{\circ} \mathrm{C}$ vs. linear PE standard. Here, $\bigoplus_{M}$ is the polydispersity index $\left(=M_{\mathrm{w}} / M_{\mathrm{n}}\right) \mathbf{k}$ In the absence of MMA. $50 \mathrm{~mL}$ of toluene was used as a solvent. 


\section{Tacticity determination of PMMA in PE/PMMA mixtures.}

Due to an overlap of the $\mathrm{mm}$ triad with the PE backbone, it was not possible to determine the tacticity from ${ }^{1} \mathrm{H}$ NMR data in analogy to the PMMA homopolymers. Therefore, the quaternary backbone ${ }^{13} \mathrm{C}$ resonances $\left(45.0-46.5 \mathrm{pmm}\right.$ in TCE- $\left.d_{2}\right)$ were used for triad assignments (see Figure S 26) ${ }^{29}$ and the tacticity was calculated according to equation 18.

$$
\% r r=\frac{I_{r r}}{\left(I_{m m}+I_{m r}+I_{r r}\right)}
$$
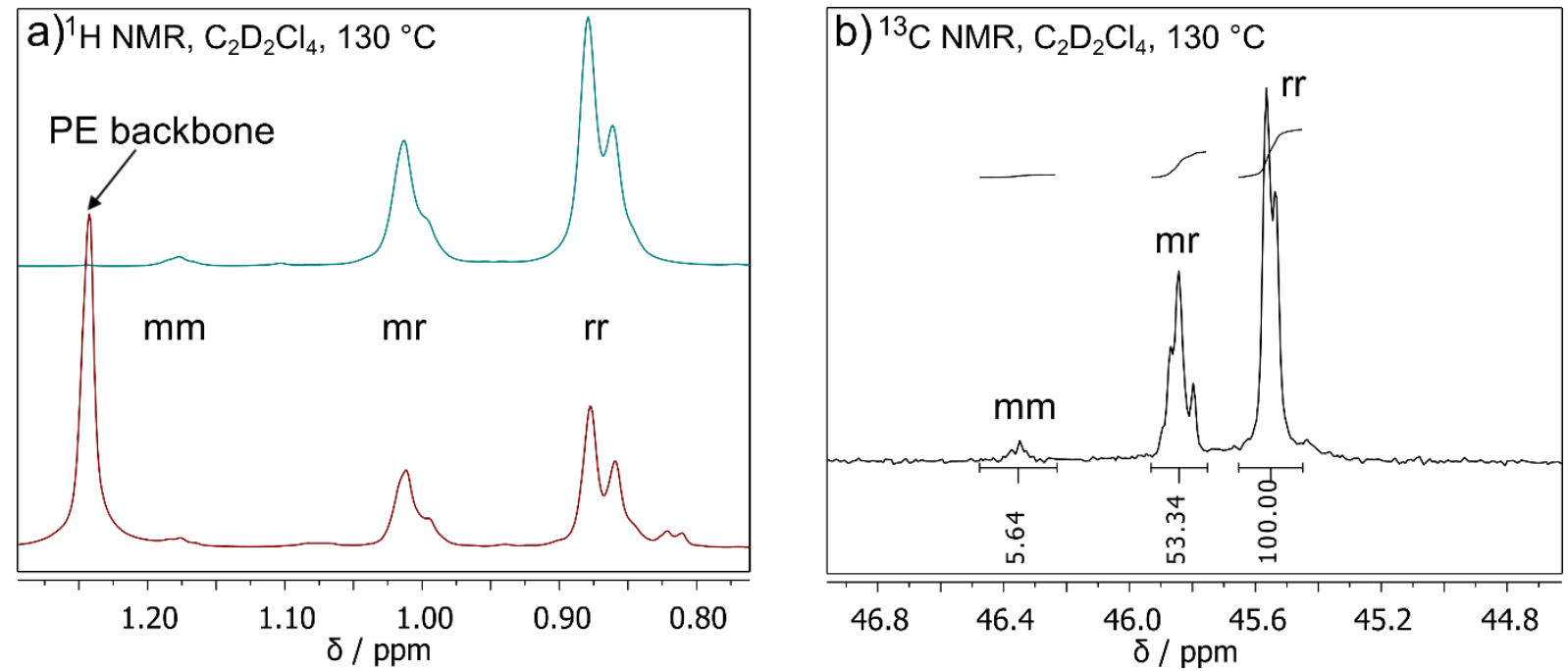

Figure S 26. (a) ${ }^{1} \mathrm{H}$ NMR $\left(400 \mathrm{MHz}, \mathrm{TCE}-d_{2}, 130{ }^{\circ} \mathrm{C}\right)$ spectra of pure PMMA (turquoise) and a PE/PMMA mixture (red) showing the overlap of the PE backbone with the mm triad of the $\alpha$-methyl group. (b) ${ }^{13} \mathrm{C}$ NMR (101 $\mathrm{MHz}, \mathrm{TCE}-\mathrm{d}_{2}, 130^{\circ} \mathrm{C}$ ) spectra of a PE/PMMA polymer mixture showing the assignment ${ }^{29}$ of the isolated quaternary PMMA backbone triads. 
Differential Scanning Calorimetry (DSC).

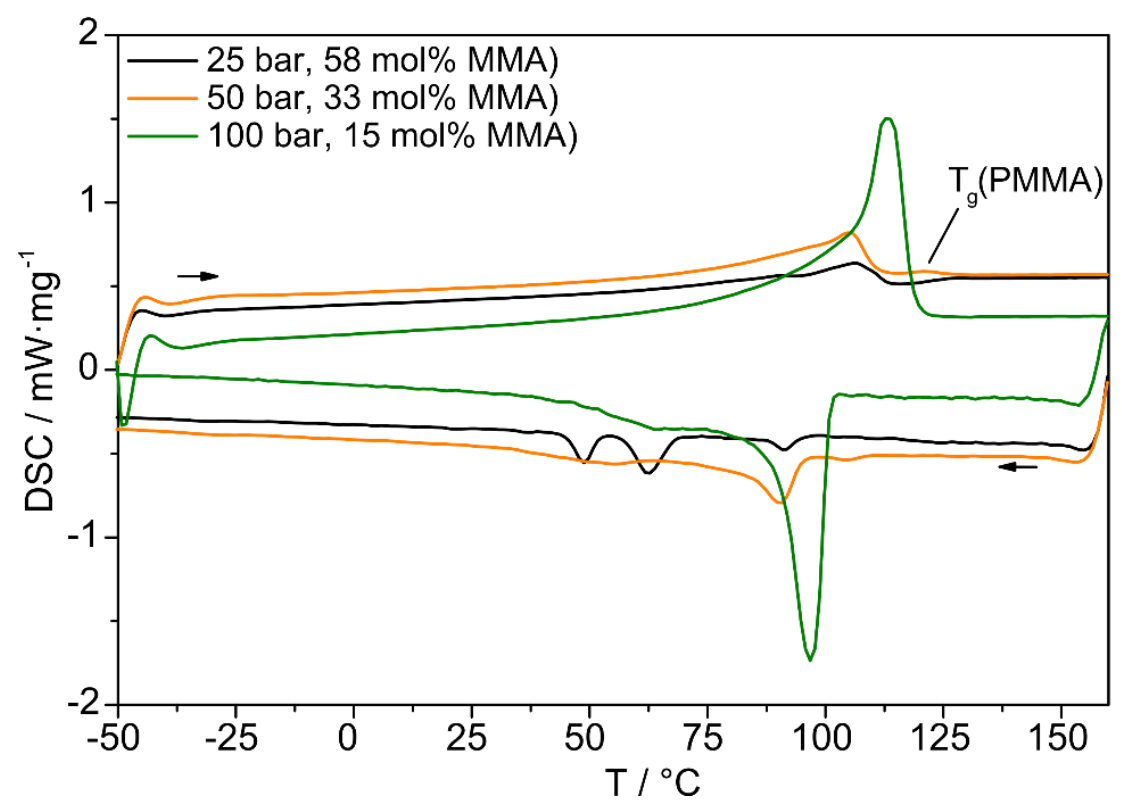

Figure S 27. Second heating and first cooling traces from DSC (exotherm down) of PE/PMMA polymer mixtures obtained with $\mathbf{1}_{\mathrm{PPh} 3}(\mathrm{R}=\mathrm{Me}$ or $\mathrm{Ph}$ ) at $25 \mathrm{bar}$ (58 mol-\% MMA, black), 50 bar (33 mol-\% MMA, orange) and 100 bar (15 mol-\% MMA, green) ethylene.

Degree of branching for PE polymers.

The degree of branching was determined by ${ }^{13} \mathrm{C}$ NMR spectroscopy by integrating the corresponding branching signals ${ }^{30}$ vs. the total signal intensity of all $\mathrm{PE}{ }^{13} \mathrm{C}$ resonances and is

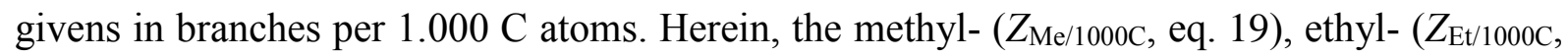
eq. 20) and the propyl- $\left(Z_{\mathrm{Pr} / 1000 \mathrm{C}}\right.$, eq. 21$)$ branching degree was determined. Butyl- and higher branches were not detected.

$$
\begin{aligned}
& Z_{M e / 1000 C}=\frac{\left(\frac{I_{\alpha \delta^{+} B_{1}}+I_{\beta \delta^{+} B_{1}}}{4}\right)}{I_{t o t}} \cdot 1000 \\
& Z_{E t / 1000 C}=\frac{\left(\frac{I_{\alpha \delta^{+} B_{2}}+I_{\beta \delta^{+} B_{2}}}{4}\right)}{I_{t o t}} \cdot 1000 \\
& Z_{P r / 1000 C}=\frac{\left(\frac{I_{\alpha \delta^{+} B_{3}}+I_{\beta \delta^{+} B_{3}}}{4}\right)}{I_{t o t}} \cdot 1000
\end{aligned}
$$

Analysis of the PE microstructure by ${ }^{13} \mathrm{C}$ NMR (for examples see Figure S 28, Figure S 29) spectroscopy revealed, that PE formed with $\mathbf{1}^{\mathrm{Me}} \mathrm{PPh}_{3}$ (Table $\mathrm{S} 7$, Entries 1-5) has a lower branching degree than PE obtained by catalysis with complexes of the type $2^{\mathrm{R}}{ }_{\mathrm{PPh} 3}(\mathrm{R}=\mathrm{Me}, \mathrm{Ph})$ (Table S 7, Entries 6-15). The degree of branching was slightly higher when MMA was used 
as a solvent $(32 / 1000 \mathrm{C})$ in comparison to polymerization in less coordinating toluene as a solvent $(25 / 1000 \mathrm{C}$, Table S 7 , entry 18 vs. 19$)$.

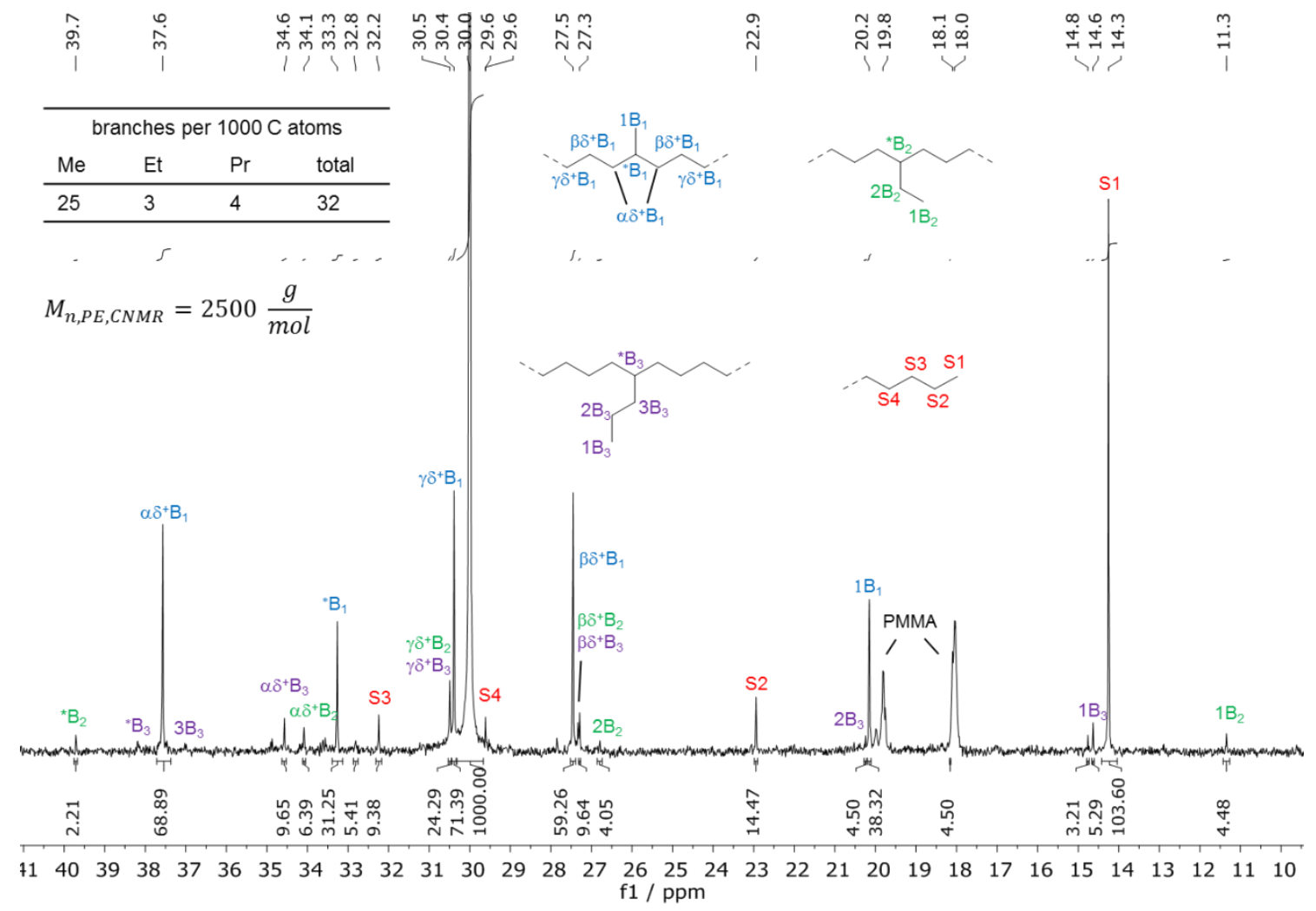

Figure S 28. Aliphatic region of the ${ }^{13} \mathrm{C}\left\{{ }^{1} \mathrm{H}\right\}$ NMR spectrum $\left(151 \mathrm{MHz}\right.$, TCE- $d_{2}, 130{ }^{\circ} \mathrm{C}, 2.0 \mathrm{~s}$ relaxation delay, $1.0 \mathrm{~s}$

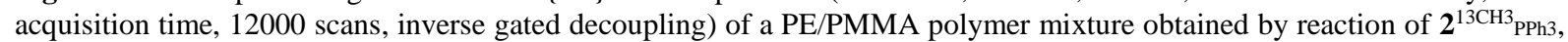
3 equiv. of $\mathrm{PPh}_{3}, 25$ bar of ethylene, $50 \mathrm{~mL}$ of MMA after 10 min polymerization time at $70{ }^{\circ} \mathrm{C}$.

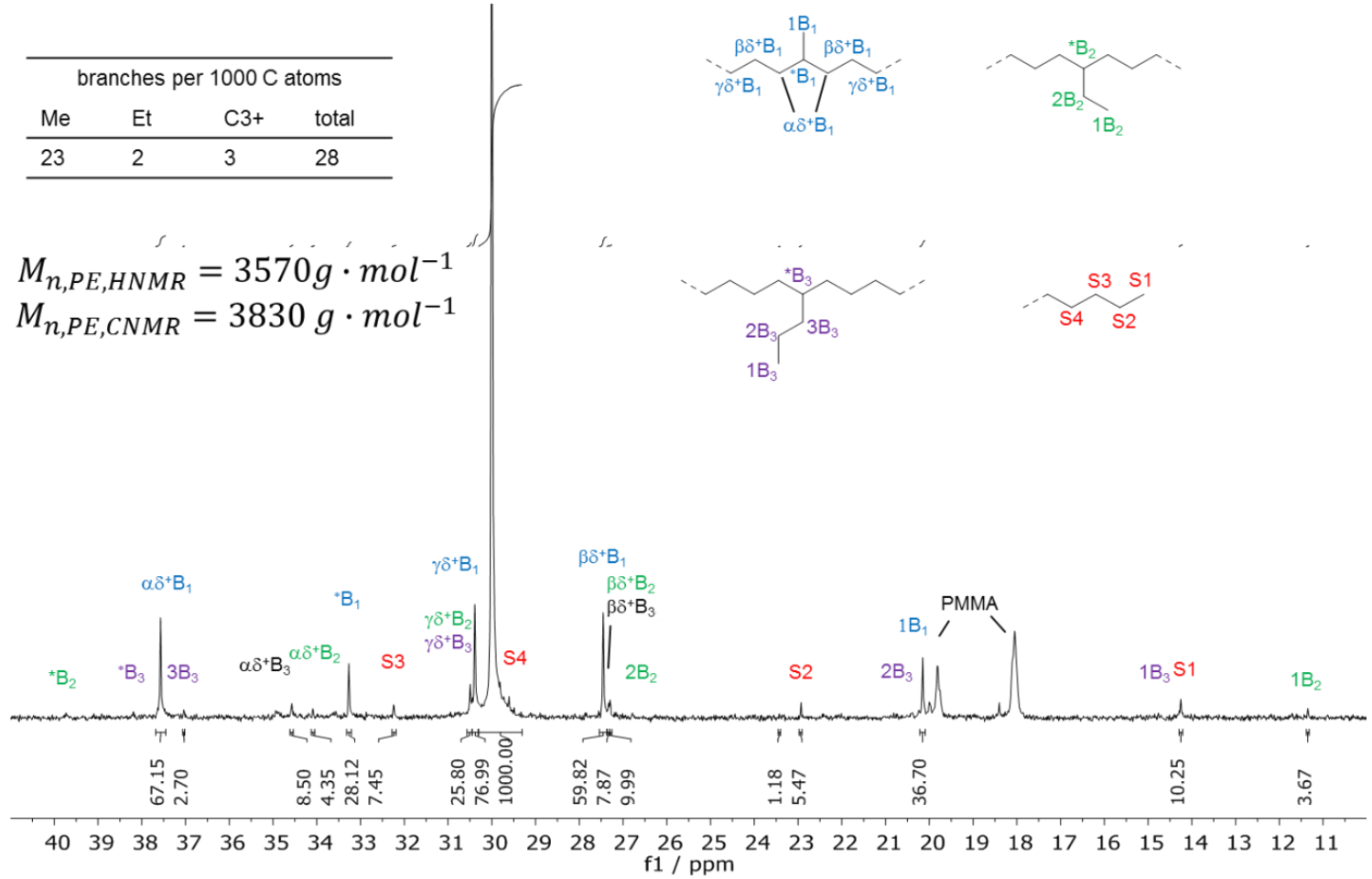

Figure $\mathbf{S} 29 .{ }^{13} \mathrm{C}\left\{{ }^{1} \mathrm{H}\right\}$ NMR spectrum $\left(151 \mathrm{MHz}, \mathrm{TCE}-d_{2}, 130{ }^{\circ} \mathrm{C}, 2.0 \mathrm{~s}\right.$ relaxation delay, $1.0 \mathrm{~s}$ acquisition time, 12000 scans, inverse gated decoupling) of a PE/PMMA polymer mixture obtained by reaction of $\mathbf{2}^{\mathrm{Ph}}{ }_{\mathrm{PPh} 3}, 3$ equiv. of $\mathrm{PPh}_{3}, 25$ bar of ethylene, $50 \mathrm{~mL}$ of MMA after 5 min polymerization time at $70{ }^{\circ} \mathrm{C}$. 


\subsection{DOSY, SEC and extraction experiments of polymers obtained from}

simultaneous polymerization of MMA and ethylene

The microstructure and end-group analysis of the polymer products formed during simultaneous ethylene and MMA polymerizations rather indicated a formation of homo-PE and homo-PMMA mixtures instead of block-co-polymers of both monomers. To clarify this issue, diffusion ordered spectroscopy (DOSY) at high temperatures is a suitable tool. ${ }^{31}$ For selected polymer mixture samples obtained with $\mathbf{1}^{\mathrm{Me}}{ }_{\mathrm{PPh}}$ (Figure S 30) and $\mathbf{2}^{\mathrm{Me}}{ }_{\mathrm{PPh}}$ (Figure S 31) two clearly separated diffusion traces were observed for the characteristic PE and PMMA backbone resonances by DOSY.

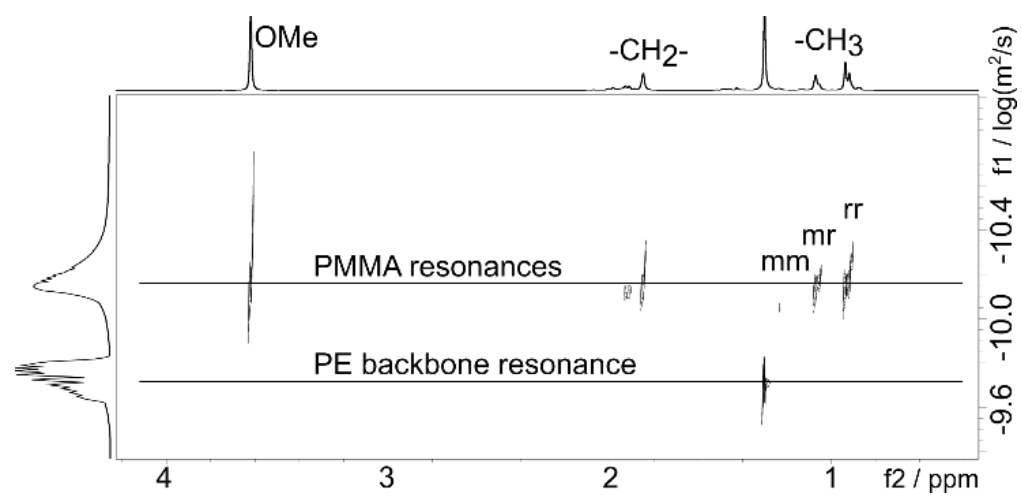

Figure S 30. ${ }^{1} \mathrm{H}$ DOSY $\left(600 \mathrm{MHz}, 130{ }^{\circ} \mathrm{C}, 1,1,2,2\right.$-tetrachloroethane- $d_{2}$ ) of a polymer mixture (52 mol\% MMAbased repeat units) polymerized with $\mathbf{1}^{\mathrm{Me}}{ }_{\mathrm{PPh}}$ plus 3 equiv. of $\mathrm{PPh}_{3}$ in $50 \mathrm{~mL}$ of MMA, 25 bar of $\mathrm{E}, 70{ }^{\circ} \mathrm{C}$ and 30 min reaction time. PMMA resonances $\left(\mathrm{D}=7.8 \cdot 10^{-10} \mathrm{~m}^{2} \cdot \mathrm{s}^{-1}\right)$ and PE backbone resonance $\left(\mathrm{D}=2.0 \cdot 10^{-10} \mathrm{~m}^{2} \cdot \mathrm{s}^{-1}\right)$ show separate diffusion traces.

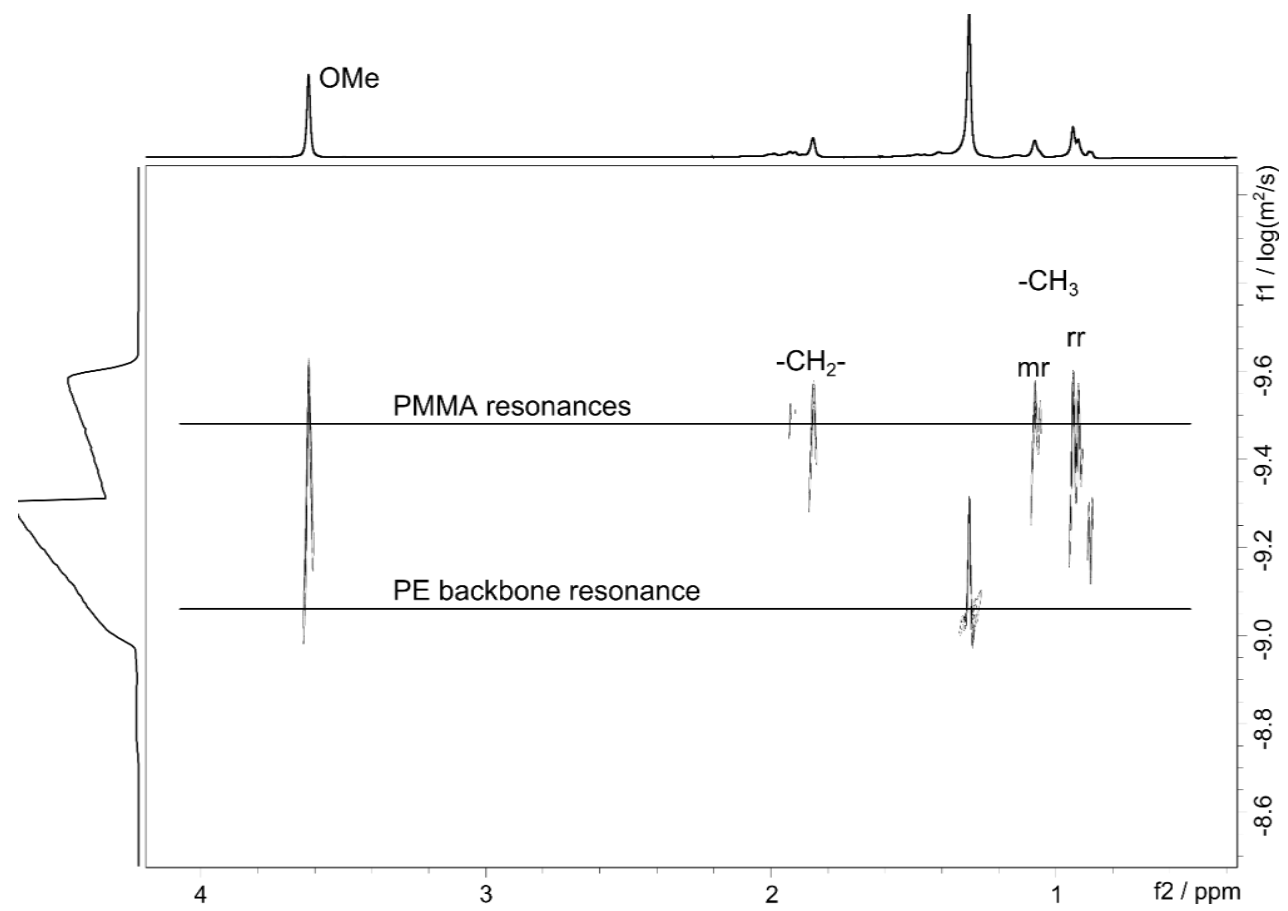

Figure S 31. ${ }^{1} \mathrm{H}$ DOSY $\left(600 \mathrm{MHz}, 130{ }^{\circ} \mathrm{C}, 1,1,2,2\right.$-tetrachloroethane- $\left.d_{2}\right)$ of a polymer (47 mol\% MMA) obtained with $2^{\mathrm{Me}}{ }_{\mathrm{PPh} 3}, 3$ equiv. of $\mathrm{PPh}_{3}$ in $50 \mathrm{~mL}$ of MMA, 25 bar of ethylene, $70^{\circ} \mathrm{C}$ and 60 min reaction time (Table $\mathrm{S} 7$ Entry 9). PMMA resonances $\left(\mathrm{D}=3.2 \cdot 10^{-10} \mathrm{~m}^{2} / \mathrm{s}\right)$ and $\mathrm{PE}$ backbone resonance $\left(\mathrm{D}=8.5 \cdot 10^{-10} \mathrm{~m}^{2} / \mathrm{s}\right)$ show separate diffusion traces. 
SEC at $160{ }^{\circ} \mathrm{C}$ in 1,2,4-trichlorobenzene is a standard method for determining molecular weights of non-polar PE polymers. However, this method was shown to be unsuitable for PE/PMMA mixtures, since the high molecular weight PMMA fraction could not be detected by the differential refractive index detection (Figure S 32 black), but with a viscosity detection (Figure S 32 red). In the linear operation mode only the response of the refractive index detection is used to determine a molecular weight, therefore, a misleading monomodal polymer distribution was obtained showing only the contribution of the PE fraction.

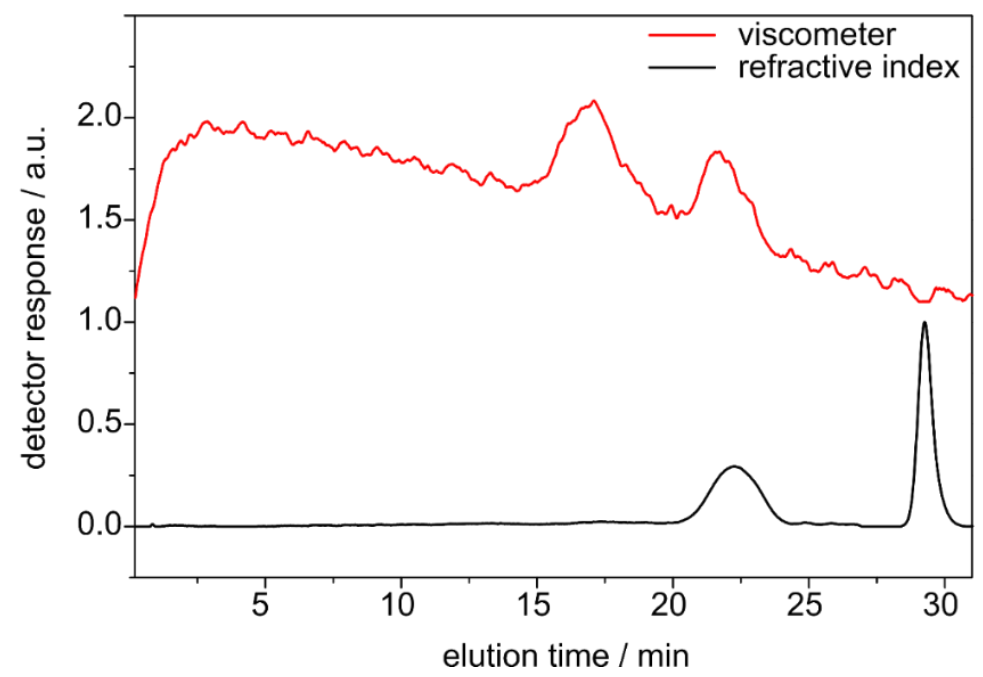

Figure S 32. HT-SEC $\left(160^{\circ} \mathrm{C}, 1,2,4\right.$-trichlorobenzene $)$ traces of a polymer mixture obtained with $2^{\mathrm{Me}}{ }_{\mathrm{PPh} 3}$ and 3 equiv. of $\mathrm{PPh}_{3}$ in $50 \mathrm{~mL}$ of MMA at 25 bar ethylene pressure at $70^{\circ} \mathrm{C}$ after $2 \mathrm{~h}(48 \mathrm{~mol} \% \mathrm{MMA})$. Refractive index response (black) is only observed for a PE polymer fraction (22 min retention time). The viscosity detector (red) shows an additional response for a higher molecular weight polymer fraction (17 min elution time). The low molecular weight response at about 28 minutes belongs to BHT addition in the solvent. 

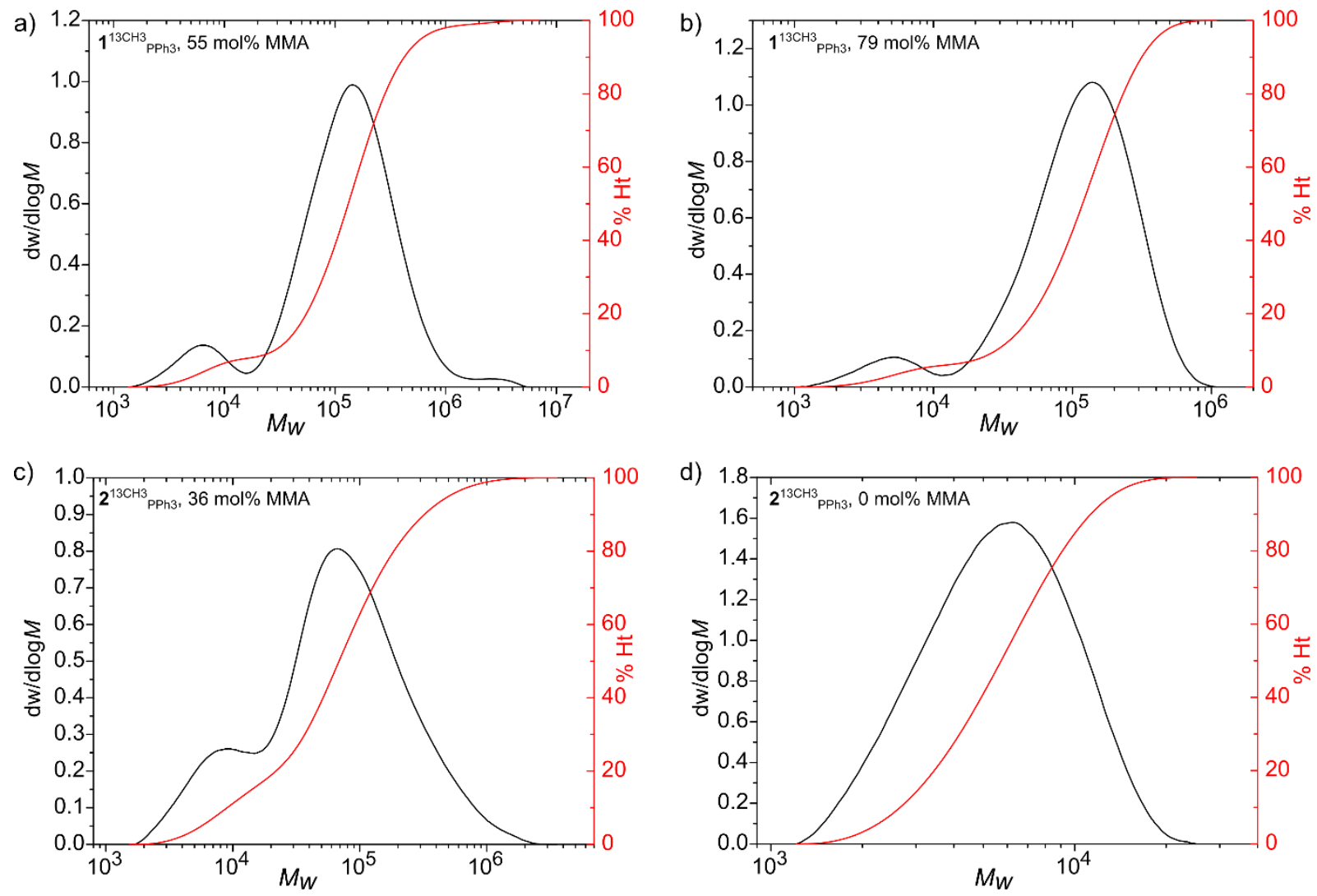

Figure S 33. SEC $\left(40{ }^{\circ} \mathrm{C}\right.$, THF vs. polystyrene standards, $M_{\mathrm{w}}$ in $\left.\mathrm{g} \cdot \mathrm{mol}^{-1}\right)$ traces of polymers obtained with $\mathbf{1}^{13 \mathrm{CH} 3}{ }_{\mathrm{PPh} 3}$ (a, b) and with $2^{13 \mathrm{CH}_{3}}{ }_{\mathrm{PPh} 3}\left(\mathbf{c}\right.$ and d) Reactions were carried out at $70^{\circ} \mathrm{C}$ at 25 bar ethylene pressure. Polymerizations $\mathbf{a}, \mathbf{b}$ and $\mathbf{c}$ were carried out in $50 \mathrm{~mL}$ MMA, whereas polymerization $\mathbf{d}$ was conducted in neat toluene in the absence of MMA. A bimodal distribution for all polymer mixtures containing PMMA was observed. The low molecular weight peak was assigned to PE (in accordance with NMR spectroscopic $M_{n}$ determination) and the high molecular weight response was assigned to PMMA. The derived $M_{\mathrm{n}}$ and $M_{\mathrm{w}} / M_{\mathrm{n}}$ values are summarized in Table S 7 .

PE/PMMA mixtures were measured by SEC at $40{ }^{\circ} \mathrm{C}$ in THF, here both polymers were detectable. Bimodal polymer distributions were observed by SEC $\left(40^{\circ} \mathrm{C}\right.$ in THF vs. PS standards, Figure S 33, Table S 7) for all polymer mixtures obtained from simultaneous ethylene and MMA polymerizations. Here, it has to be mentioned that polymers obtained with catalyst precursors $\mathbf{2}^{\mathrm{Ph}}{ }_{\mathrm{PPh} 3}$ and $\mathbf{2}^{\mathrm{Me}}{ }_{\mathrm{PPh} 3}$ were completely soluble in THF, whereas polymers obtained with $\mathbf{1}^{\mathrm{Me}} \mathrm{PPh}$ showed a slightly lower PE branching content and a turbid suspension was obtained. The non-soluble parts were separated using a syringe filter. Due to solubility issues SEC at $40{ }^{\circ} \mathrm{C}$ in THF is limited to low molecular weight PE with a high branching content. The low molecular weight fractions $\left(3.000-6.000 \mathrm{~g} \cdot \mathrm{mol}^{-1}, M_{\mathrm{w}} / M_{\mathrm{n}} \sim 1.2-1.3\right)$ were assigned to PE homopolymers and the high molecular weight fractions to PMMA $\left(60.000-110.000 \mathrm{~g} \cdot \mathrm{mol}^{-1}\right.$, $\left.M_{\mathrm{w}} / M_{\mathrm{n}} \sim 2-3\right)$. The fractions were assigned by comparison with $M_{\mathrm{n}}$ determined by ${ }^{1} \mathrm{H}$ NMR spectroscopy. Here it has to be considered that SEC vs. PS standards overestimates the $M_{\mathrm{n}}$ of highly branched PE by a factor of approximately two as was previously reported by Wiedemann et al. ${ }^{30 \mathrm{c}}$ This assignment of the polymer fractions was further corroborated by extraction of a polymer mixture obtained with $\mathbf{1}^{\mathrm{Me}}{ }_{\mathrm{PPh} 3}\left(25\right.$ bar of $\mathrm{E}, 70{ }^{\circ} \mathrm{C}, 3$ equiv. of $\left.\mathrm{PPh}_{3}, 52 \mathrm{~mol}-\% \mathrm{MMA}\right)$ 
with non-polar cyclohexane (CyHex) and analysis of the soluble and insoluble fractions by DSC and SEC (Table S 8, Figure S 34).

Table S 8. Extraction experiments of PE/PMMA blends and a polymer obtained by [Ni] mediated simultaneous ethylene and MMA polymerization with non-polar cyclohexane (CyHex).

\begin{tabular}{|c|c|c|c|c|c|c|}
\hline Entry & polymer & $\begin{array}{l}\text { wt-\% MMA } \\
\text { (MMA cont. } \\
\text { mol-\% } \%^{\text {b) }}\end{array}$ & solvent & $\begin{array}{l}\text { Extr. } \\
\text { time } \\
\text { / h }\end{array}$ & $\begin{array}{l}\text { wt-\% soluble } \\
\text { (MMA cont. } \\
\text { mol-\% } \% \text { ) }\end{array}$ & $\begin{array}{l}\text { wt-\% insoluble } \\
\text { (MMA cont. } \\
\text { mol-\%b) }\end{array}$ \\
\hline 1 & PE/PMMA blend ${ }^{\mathrm{a}}$ & $21.8(7.2)$ & CyHex & 16 & $75.8(<0.01)$ & $24.2(90.9)$ \\
\hline 2 & Table S 7 Entry 3 & $79.4(52.0)$ & CyHex & 24 & $20.5(0.3)$ & $79.5(89.1)$ \\
\hline
\end{tabular}

Extractions were conducted in a soxhlet apparatus. a Homopolymers of PE and PMMA obtained by [Ni] mediated polymerizations were dissolved in boiling toluene and precipitated in an excess of $\mathrm{MeOH}$ prior to extraction experiments. The molecular weights of both homopolymers are in the range of the expected $M_{\mathrm{n}}$ for the polymers in the polymerization mixture obtained by simultaneous E/MMA polymerization mediated by [Ni] complexes. b Determined by HT ${ }^{1} \mathrm{H}$ NMR in TCE- $d_{2}$.

a)

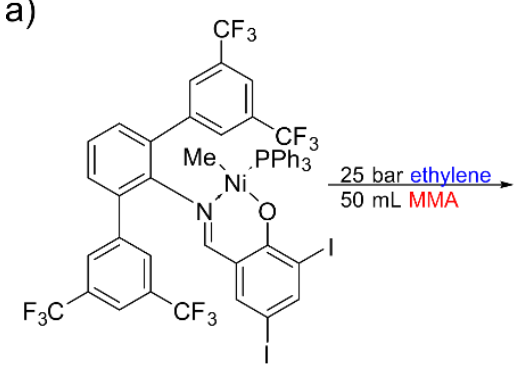

b)

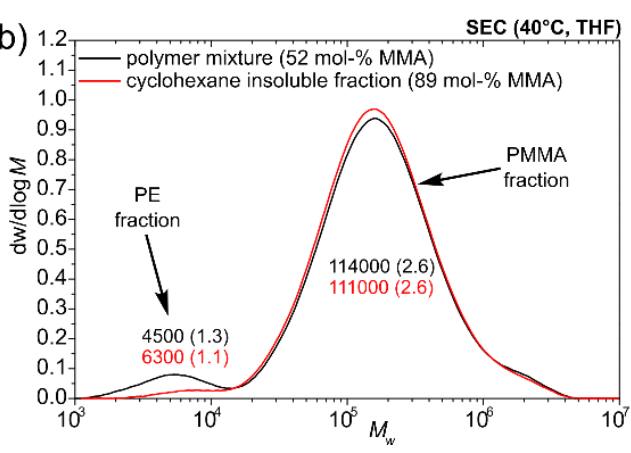

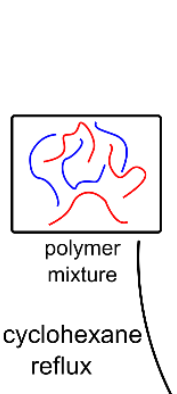

.

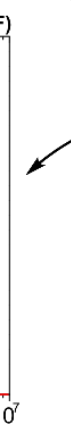

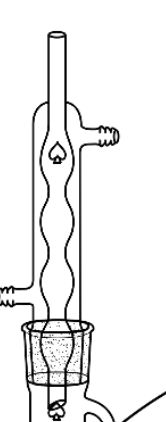

$$
\text { c) }
$$

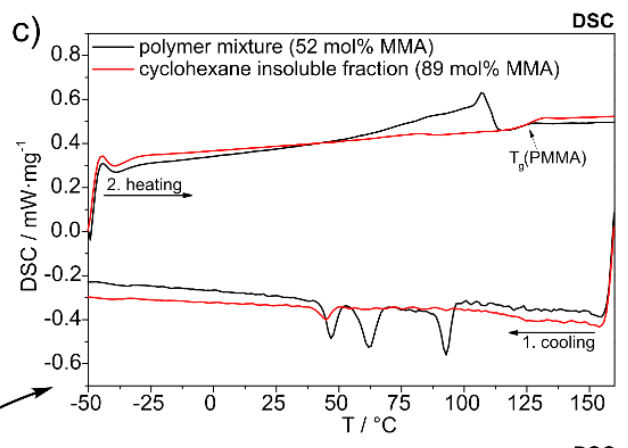

d)

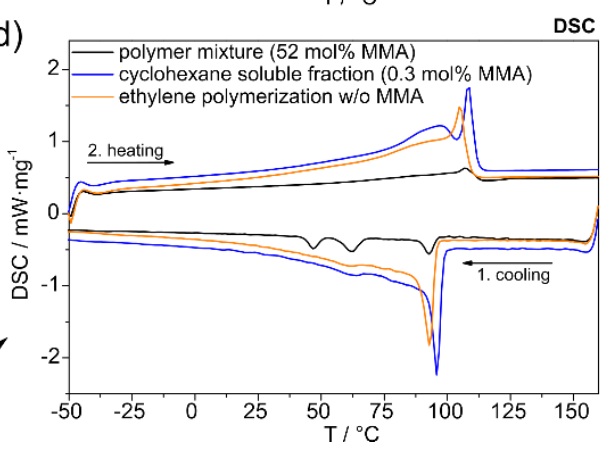

Figure S 34. (a) Schematic representation of the extraction process of a polymer mixture obtained by polymerization with $\mathbf{1}^{\mathrm{Me}}{ }_{\mathrm{PPh}}$ at 25 bar of ethylene in $50 \mathrm{~mL}$ of neat MMA at $70{ }^{\circ} \mathrm{C}$ (Table S 7, Entry 3). (b) SEC $\left(40{ }^{\circ} \mathrm{C}\right.$, THF vs. PS standard) traces of the polymer mixture obtained after polymerization (52 mol\% MMA, black) and of the cyclohexane insoluble fraction after extraction ( $89 \mathrm{~mol} \%$ MMA, red). The low molecular weight fractions were assigned to PE and the high molecular weight fractions to PMMA homopolymers. (c) DSC traces for the polymer mixtures after polymerization (black) and of the cyclohexane insoluble fraction ( 89 mol\% MMA, red). (d) DSC traces for the polymer mixtures after polymerization (black), after extraction with CyHex $(0.3 \mathrm{~mol} \%$ MMA, blue). For comparison, a DSC trace for a PE homopolymer obtained under similar conditions in the absence of MMA is also given (orange).

Extraction of a polymer obtained with $\mathbf{1}^{\mathrm{Me}}{ }_{\mathrm{PPh} 3}$ (Table S 8-2, 52 mol-\% MMA, 79 wt-\% MMA) with non-polar cyclohexane afforded an extract ( $21 \mathrm{wt}-\%)$ with a very low MMA content of 0.3 mol-\%. DSC traces of this soluble fraction display thermal behavior of pure PE (Figure S 34d). The remaining insoluble fraction was containing 89 mol-\% MMA based repeat units, mainly 
resembling the characteristics of pure PMMA (DSC, Figure S 34c). For this insoluble fraction a bimodal polymer distribution was observed by SEC (Figure S $34 \mathrm{~b}, 40{ }^{\circ} \mathrm{C}$, THF vs. PS standard) with a weak response, in comparison to the polymer mixture before extraction, of a low $M_{\mathrm{n}}$ PE fraction $\left(6300 \mathrm{~g} \cdot \mathrm{mol}^{-1}\right)$ and a high $M_{\mathrm{n}}$ PMMA fraction $\left(110 \cdot 10^{3} \mathrm{~g} \cdot \mathrm{mol}^{-1}\right)$.

Determination of the ratio of saturated and unsaturated end-groups in PE polymers obtained from simultaneous ethylene and MMA polymerizations.

This calculation assumes that polymers very closely to PMMA homopolymers were formed, which was shown by the aforementioned polymer analytic (SEC, DOSY, extraction experiments). In addition, molecular weights of the PMMA fractions are required. These were obtained by SEC at $40^{\circ} \mathrm{C}$ in THF vs. PS standards (Table S 7).

The ratio of saturated end-groups (initiation of insertion chain growth of ethylene, corrected for phenyl end-groups, where applicable, $\left.{ }^{13} \mathrm{C} \mathrm{NMR}, I_{\mathrm{n}, \mathrm{sat}}\right)$ to unsaturated end-groups $(\beta$-H elimination, ${ }^{1} \mathrm{H}$ NMR, $I_{\mathrm{n}}$,unsat) in the PE fraction was calculated by dividing the corresponding corrected intensities (vide supra), which were normalized to the repeating units of the polymer chain. The intensity for the unsaturated end-groups ( $\left.I_{\mathrm{n} \text {,unsat }}\right)$ was therefore derived by eq. 22 and for the saturated end-groups according to eq. 23.

$$
\begin{aligned}
& I_{n, \text { unsat }}=\frac{\left(\frac{I_{\text {aliph }}-\left(I_{\text {OMe }} \cdot \frac{5}{3}\right)}{4}\right)}{\left(\frac{I_{1}+I_{2}+2 I_{3}+2 I_{4}+I_{5}}{2}\right)} \\
& I_{n, \text { sat }}=\frac{I_{\text {tot }}}{I_{\text {end-group }}}
\end{aligned}
$$

Also, the fraction ( $\left.N_{\text {unsat }} / N_{\text {sat }}\right)$ of the end-groups which were introduced by $\beta$-H elimination vs. the initiation of a chain growth by insertion into the catalyst precursors $[\mathrm{Ni}-\mathrm{Me}] /[\mathrm{Ni}-\mathrm{Ph}]$ or the reactive intermediate $[\mathrm{Ni}-\mathrm{H}]$ species, was calculated by eq. 24 .

$$
\frac{N_{\text {unsat }}}{N_{\text {sat }}}=\frac{I_{n, \text { unsat }}}{I_{n, \text { sat }}}
$$

Finally, in the present study a ratio of $N_{\text {unsat }}$ to $N_{\text {sat }}$ of close to or one was found for polymerizations in the presence or the absence of MMA (see main text). From this observation it was concluded that MMA does not influence the elemantary steps of the migratory insertion chain growth polymerization. In principle, an effective transfer of the growing migratory insertion species [Ni-PE] to PE polymeryl radicals, a subsequent radical chain growth of MMA, 
addition of the PE-PMMA radical to [Ni], following a migratory insertion polymerization of ethylene with [Ni-PMMA-PE] and selective $\beta$-H elimination from [Ni-PE-PMMA-PE] could lead to the same end-group ratio. However, this scenario can be excluded by comparison with the SEC, DOSY and extraction experiments. 


\section{Stoichiometric Reactions of $\left(\mathrm{N}^{\wedge} \mathrm{O}\right) \mathrm{Ni}(\mathrm{II})-$ Catalyst Precursors and Intermediate [Ni]-Species toward MMA.}

\subsection{General procedure for NMR experiments.}

Samples were weighted in a $\mathrm{N}_{2}$ filled glovebox. For experiments conducted at low temperature, the tube was sealed with a rubber septum, removed from the glovebox and cooled in an $i \mathrm{PrOH} / \mathrm{CO}_{2}$ (s) cooling bath. Afterwards $0.6 \mathrm{~mL}$ of the corresponding dried and degassed deuterated solvent was added slowly using a gas-tight syringe, the rubber septum was wrapped with PTFE tape and the tube was agitated thoroughly. The sample was placed directly in the precooled NMR probe at the desired temperature. Samples for RT and/or high temperature experiments were filled with $0.6 \mathrm{~mL}$ dried and degassed deuterated solvent using a syringe in a $\mathrm{N}_{2}$ glovebox, sealed with a rubber septum, removed from the glovebox, the rubber septum was wrapped with PTFE tape, the tube was agitated thoroughly and transferred quickly into the preheated NMR probe. For very sensitive samples and especially for long-term experiments J. Young Tubes with Teflon sealing were used. All samples were stored at $-78{ }^{\circ} \mathrm{C}$ when outside the spectrometer. For variable temperature experiments, temperature equilibration time was respected and tuning/matching and shimming was performed for each temperature step. Temperature calibration was carried out by using a neat methanol sample for $\mathrm{T} \leq 40{ }^{\circ} \mathrm{C}$ and ethylene glycol for $\mathrm{T} \geq 40{ }^{\circ} \mathrm{C}$.

\section{Reaction of $\left[\left(N^{\wedge} O\right) N i(I I) R(L)\right]$ catalyst precursors with ethylene.}

1) The desired amount of catalyst precursor was weighted into a NMR tube in a $\mathrm{N}_{2}$ filled glovebox and the desired amount of deuterated solvent was added via syringe. The NMR tube was sealed with a rubber septum and removed from the glovebox. The desired amount of ethylene was added using a gas tight syringe. The rubber septum was wrapped with PTFE tape, the tube was agitated thoroughly and quickly transferred into the preheated NMR probe.

2) The desired amount of catalyst precursor was weighted into a J. Young Tube under $\mathrm{N}_{2}$ atmosphere in a glovebox. The desired amount of deuterated solvent was added and the tube was removed from the glovebox, connected to a Schlenk-line with a connection to an ethylene bottle and pressurized with 1.2 bar ethylene. The mixture was allowed to react at the desired temperature for several hours and was again pressurized with ethylene until the desired degree of conversion was detected by ${ }^{1} \mathrm{H}$ NMR monitoring. 


\subsection{Additional discussion of the observed reactivity of $\left(N^{\wedge} \mathrm{O}\right) \mathrm{Ni}$ species by} spectroscopic methods

\section{Reactivity of $\left[\left(N^{\wedge} O\right) N i(I I) P h\left(P P h_{3}\right)\right]$ toward ethylene}

Scheme S 5. Reaction of $3^{\mathrm{Ph}}{ }_{\mathrm{PPh} 3}$ with ethylene, reaction products and proposed pathways for their formation. Labile ligands are omitted.

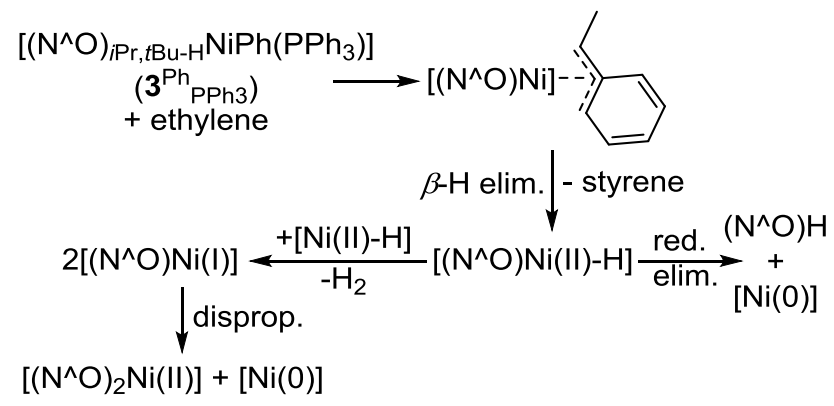

As they are the most common $\left(\mathrm{N}^{\wedge} \mathrm{O}\right) \mathrm{Ni}(\mathrm{II})$ catalyst precursor complexes for the insertion polymerization of ethylene, we focused on the reactivity of [Ni-Ph] complexes toward ethylene.

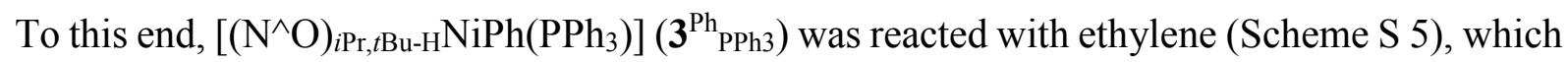
yielded an observed $\eta^{3}$-benzyl Ni(II) complex (15 equiv. of ethylene, $6{ }^{\circ} \mathrm{C}, 72 \mathrm{~h} \mathrm{C}_{6} \mathrm{D}_{6}$, Figure S 37 and Figure S 38). Further reaction via $\beta$-H elimination led to the observed formation of styrene and the corresponding $[\mathrm{Ni}-\mathrm{H}]$ species $\left(3^{\mathrm{H}} \mathrm{Pph} 3, \delta\left({ }^{1} \mathrm{H}\right)=-27.15 \mathrm{ppm}\right.$, Figure S 39). In addition, 1-butene and 2-E/Z-butene from dimerization reactions, and protonated $\left(\mathrm{N}^{\wedge} \mathrm{O}\right)$ ligand were detected. The latter was presumably formed by reductive elimination from intermediate $[\mathrm{Ni}-\mathrm{H}]$ species to the salicylaldiminato ligand, associated with the expected formation of a $[\mathrm{Ni}(0)]$ species. Previously, similar decomposition reactions for other $[\mathrm{Ni}-\mathrm{H}]$ species were described by Jenkins et al. and Waltman et al. ${ }^{32}$

In addition to the organic products, paramagnetic species were detected in the reaction mixture

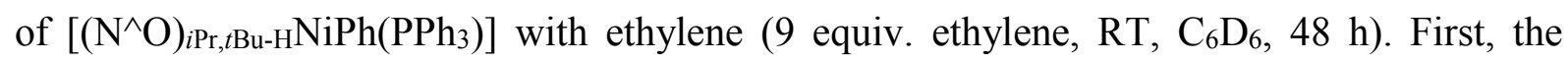
paramagnetic, tetrahedral coordinated, bis-chelated complex $\left[\left\{\left(\mathrm{N}^{\wedge} \mathrm{O}\right)_{i \mathrm{Pr}, t \mathrm{Bu}-\mathrm{H}}\right\}_{2} \mathrm{Ni}\right]$ (Figure S 12) was identified via ${ }^{1} \mathrm{H}$ NMR spectroscopy by comparison with characteristic resonances of

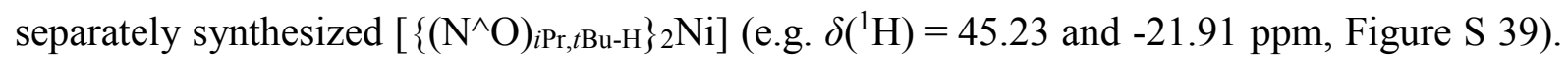
In addition, we hypothesized that an intermediate paramagnetic $\left[\left(\mathrm{N}^{\wedge} \mathrm{O}\right) \mathrm{Ni}(\mathrm{I})\right]$ complex could be formed via bimolecular reductive coupling, e.g. eliminating $\mathrm{H}_{2}$ from two [Ni-H] species (Figure S 35a). The corresponding complex, $\left[\left(\mathrm{N}^{\wedge} \mathrm{O}\right)_{i \mathrm{Pr}, t \mathrm{Bu}-\mathrm{H}} \mathrm{Ni}(\mathrm{I})\left(\mathrm{PPh}_{3}\right)_{2}\right]\left(3^{\mathrm{PPh} 3}{ }_{\mathrm{PPh} 3}\right)$, was synthesized separately (Figure S 35b). Note that it represents the first isolated and characterized $\left(\mathrm{N}^{\wedge} \mathrm{O}\right) \mathrm{Ni}(\mathrm{I})$ complex reported to date. Indeed, $\mathrm{Ni}(\mathrm{I})$ complex $3^{\mathrm{PPh} 3} \mathrm{PPh} 3$ was formed

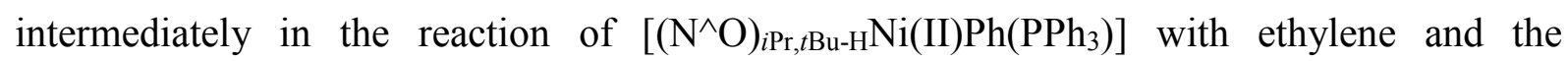


characteristic resonances could be identified via ${ }^{1} \mathrm{H}$ NMR (Figure S 39) and EPR spectroscopy

(Figure S 35c) by comparison with spectroscopic features of a separately prepared sample.

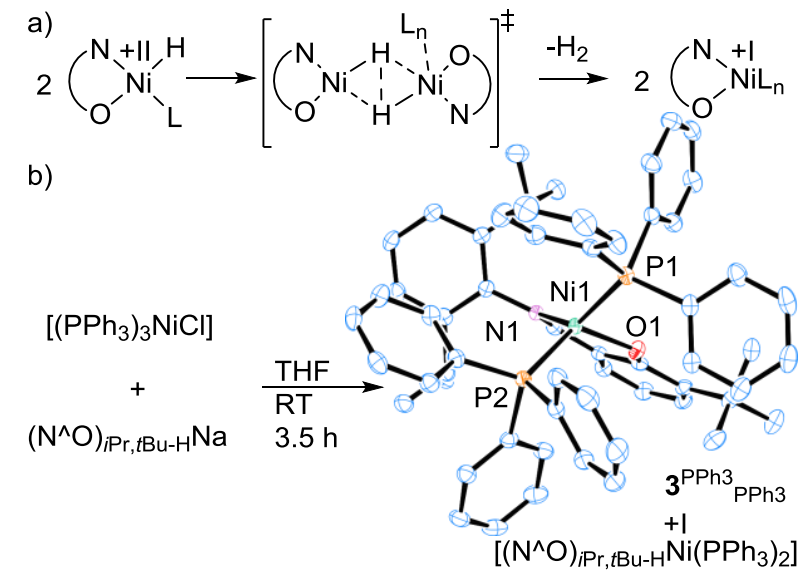

c)

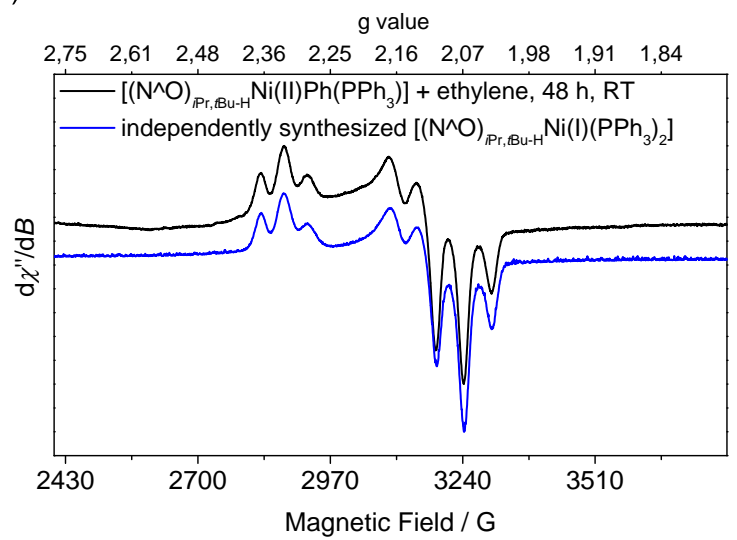

Figure S 35. Identification of the paramagnetic $[\mathrm{Ni}(\mathrm{I})]$ complex $\left[\left(\mathrm{N}^{\wedge} \mathrm{O}\right)_{i \mathrm{Pr}, t \mathrm{Bu}-\mathrm{H}} \mathrm{Ni}(\mathrm{I})\left(\mathrm{PPh}_{3}\right)_{2}\right]$ formed during the reaction of $3^{\mathrm{Ph}}{ }_{\mathrm{PPh} 3}$ with ethylene. (a) Schematic representation of the bimolecular reductive elimination leading to

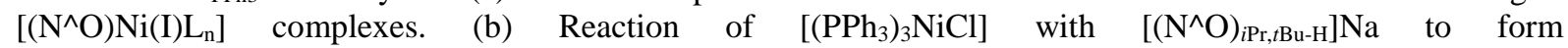
$\left[\left(\mathrm{N}^{\wedge} \mathrm{O}\right)_{i \mathrm{Pr}, t \mathrm{Bu}-\mathrm{H}} \mathrm{Ni}(\mathrm{I})\left(\mathrm{PPh}_{3}\right)_{2}\right]$, which is shown as ORTEP $(50 \%$ probability ellipsoids, hydrogens are omitted for clarity) plot determined by X-ray diffraction. (c) X band EPR spectra $\left(9.36 \mathrm{GHz}\right.$, toluene, $\left.-170{ }^{\circ} \mathrm{C}\right)$ of the reaction mixture of $3^{\mathrm{Ph}}{ }_{\mathrm{PPh} 3}(27 \mathrm{mM})$ and 9 equiv. of ethylene after $48 \mathrm{~h}$ at room temperature (black) and of separately synthesized $3^{\mathrm{PPh} 3} \mathrm{PPh} 3$ (blue).

Repeated synthetic approaches to $\mathrm{Ni}(\mathrm{I})$ complexes include their generation via intermediate formation of $[\mathrm{Ni}(\mathrm{II})-\mathrm{H}]$ species. ${ }^{13 b, 33}$ Therefore, we believe that the formation of the observed $[\mathrm{Ni}(\mathrm{I})]$ complex occurs mainly by bimolecular reaction of reactive $[\mathrm{Ni}(\mathrm{II})-\mathrm{H}]$ complexes after $\beta$-H elimination. To elucidate the role of the $[\mathrm{Ni}-\mathrm{H}]$ species more directly, $3^{\mathrm{H}} \mathrm{PPh} 3$ was generated in-situ by reaction of $3^{\mathrm{Cl}}{ }_{\mathrm{PPh} 3}$ with 3 equiv. of $\mathrm{NaHB}(\mathrm{OMe})_{3}$ in THF- $d 8$ at RT (Figure $\mathrm{S} \mathrm{40}$ ). Presumably via bimolecular reductive coupling, $3^{\mathrm{H}}{ }_{\mathrm{PPh} 3}$ reacted to $\left[\left(\mathrm{N}^{\wedge} \mathrm{O}\right) \mathrm{Ni}(\mathrm{I})\right]$ complex $3^{\mathrm{PPh} 3}{ }_{\mathrm{PPh} 3}$ and the release of $\mathrm{H}_{2}\left(\delta\left({ }^{1} \mathrm{H}\right)=4.54 \mathrm{ppm}\right)$ was observed by ${ }^{1} \mathrm{H}$ NMR spectroscopy. In addition, bis-chelated $\left[\left(\mathrm{N}^{\wedge} \mathrm{O}\right)_{2} \mathrm{Ni}(\mathrm{II})\right]$ complex $\{\mathbf{3 - 3}\}$ as the expected product formed after disproportionation of the intermediate $[\mathrm{Ni}(\mathrm{I})]$ species was identified. However, a reduced $[\mathrm{Ni}(0)]$ species should also be formed after disproportionation of a $[\mathrm{Ni}(\mathrm{I})]$ species. This was confirmed by identification of the reaction products in the attempted synthesis of

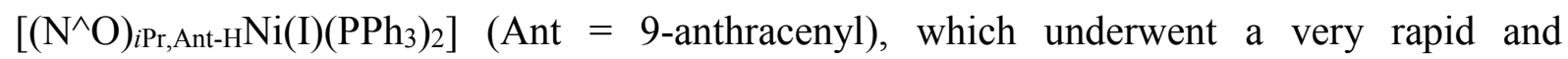
quantitative further reaction. Bis-chelated complex $\left[\left\{\left(\mathrm{N}^{\wedge} \mathrm{O}\right)_{i \mathrm{Pr}, \mathrm{Ant}-\mathrm{H}}\right\}_{2} \mathrm{Ni}\right]$ was observed by NMR spectroscopy and $\left[\mathrm{Ni}(0)\left(\mathrm{PPh}_{3}\right)_{4}\right]$ was clearly identified by addition of 3,5dimethoxybromobenzene to the reaction mixture, which resulted in the formation of the oxidative addition product, trans-bromo(3,5-dimethoxyphenyl) bis(triphenylphosphine)nickel(II) (Figure S 41).

In summary, in the presence of ethylene activation of the catalyst precursors (herein shown for $3^{\mathrm{Ph}}{ }_{\mathrm{PPh} 3}$ and previously reported in literature ${ }^{10}$ for $\mathbf{1}^{\mathrm{Me}}{ }_{\text {dmso }}$ ) leads to [Ni-alkyl] complexes, which 
can undergo $\beta-\mathrm{H}$ elimination to form reactive $[\mathrm{Ni}-\mathrm{H}]$ species. These $[\mathrm{Ni}-\mathrm{H}]$ species are prone to decomposition reactions even under mild conditions. First, reductive elimination of the $\left(\mathrm{N}^{\wedge} \mathrm{O}\right)$ ligand can occur (to form $\left.\left(\mathrm{N}^{\wedge} \mathrm{O}\right) \mathrm{H}\right)$ and second, a bimolecular reductive coupling is likely to occur. The latter was shown to proceed via intermediate metal-centered radicals $\left[\left(\mathrm{N}^{\wedge} \mathrm{O}\right) \mathrm{Ni}(\mathrm{I})\right]$ and $\mathrm{H}_{2}$ formation. Further disproportionation leads to the final decomposition products: the bis-chelated complex $\left[\left(\mathrm{N}^{\wedge} \mathrm{O}\right)_{2} \mathrm{Ni}(\mathrm{II})\right]$ and the corresponding $[\mathrm{Ni}(0)]$ species (Scheme S 6).

Scheme S 6. Bimolecular reductive coupling pathway as observed for $[\mathrm{Ni}-\mathrm{H}]$ species. Labile ligands are omitted.

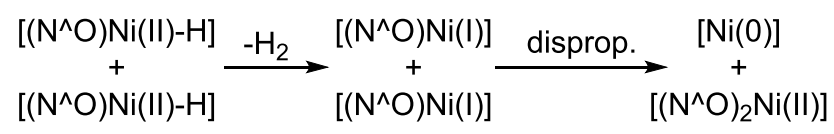

Reactivity of $\left[\left(N^{\wedge} O\right) N i(I I) R(L)\right]$ toward $M M A$.

The stoichiometric experiments of ethylene with a catalyst precursor gave valuable insights into decomposition pathways and possible [Ni] species formed during polymerization reactions in the presence of ethylene. For a better understanding of the simultaneous polymerization of ethylene and MMA, and the polymerization of MMA alone, the reactivity of catalyst precursors toward MMA is significant.

Scheme $S_{7}$. Reactivity of [Ni-Ph] complexes toward MMA and proposed formation pathways of the observed products. [( $\left.\left.\mathrm{PPh}_{3}\right)_{2} \mathrm{Ni}(\mathrm{o})\left(\eta^{2}-\mathrm{MMA}\right)\right]$ was only observed for $3^{\mathrm{Ph}} \mathrm{PPh}_{3}$. Structures in grey were not observed by NMR spectroscopy (A and $[\mathrm{Ni}-\mathrm{H}])$.

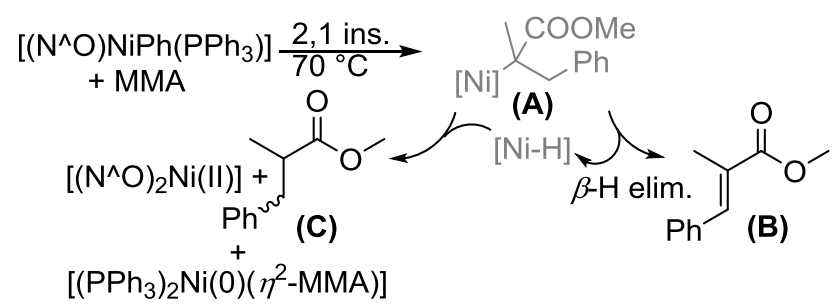

As [Ni-Ph] complexes are the most common precursor complexes applied in this chemistry, the reactivity of $\mathbf{1}^{\mathrm{Ph}}{ }_{\mathrm{PPh} 3}, \mathbf{2}^{\mathrm{Ph}}{ }_{\mathrm{PPh} 3}$ and $3^{\mathrm{Ph}}{ }_{\mathrm{PPh} 3}$ with stoichiometric amounts of MMA (3 to 7 equiv., $70{ }^{\circ} \mathrm{C}$ ) was studied by NMR spectroscopy. As the final organic reaction products, unsaturated methyl 2-methyl-(E)-3-phenyl-2-propenoate (Scheme S 7 B) and saturated methyl 2-methyl-3phenylpropionate (Scheme S 7 C) were identified in the reaction solutions (Figure S 42 - Figure S 48).

Methyl 2-methyl-(E)-3-phenyl-2-propenoate (Scheme S 7 B) was likely formed via $\beta$-H elimination from the intermediate [Ni-alkyl] complex after 2,1 insertion of MMA in the [Ni-Ph] bond (Scheme S 7 A). Methyl 2-methyl-3-phenylpropionate (Scheme S 7 C) was presumably generated via bimolecular reductive coupling of intermediate [Ni-H] species with the [Ni-alkyl] complex formed after 2,1 insertion of MMA into [Ni-Ph] (Scheme $\mathrm{S} 7 \mathbf{A}$ ). In addition, bis- 
chelated complexes were observed by ${ }^{1} \mathrm{H}$ NMR spectroscopy. In the case of

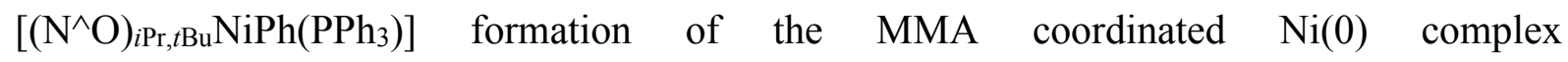
$\left[\left(\mathrm{PPh}_{3}\right)_{2} \mathrm{Ni}(0)\left(\eta^{2}-\mathrm{H}_{2} \mathrm{C}=\mathrm{C}\left(\mathrm{CH}_{3}\right) \mathrm{COOMe}\right)\right]$ was observed (Figure $\mathrm{S} \mathrm{49}$, Figure $\mathrm{S} \mathrm{50).}{ }^{34}$ The same ultimate Ni-species were observed during the decomposition of separately prepared

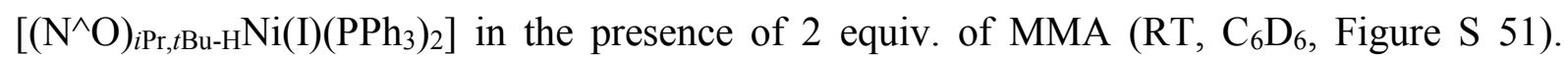
Although, no $[\mathrm{Ni}-\mathrm{H}]$ or $[\mathrm{Ni}(\mathrm{I})]$ species were directly observed by NMR spectroscopy, the aforementioned results indicate that the formation of methyl 2-methyl-3-phenylpropionate (Scheme S 7 C) proceeded via a bimolecular reductive coupling pathway with intermediate $[\mathrm{Ni}(\mathrm{I})]$ formation.

Scheme S 8. Reactivity of [Ni-Me] complexes $\left(\mathbf{1}^{\mathrm{Me}} \mathrm{L}, \mathrm{L}=\mathrm{dmso}, \mathrm{PPh}_{3}\right)$ toward MMA. No insertion of MMA was observed, instead decomposition to form ethane and $\mathrm{MePPh}_{3}{ }^{+}$occurred.

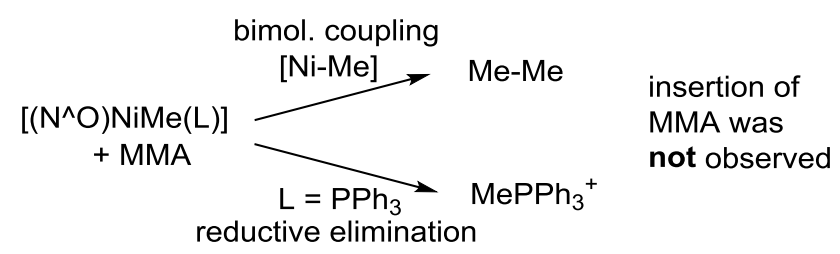

Additionally, the reaction of MMA and [Ni-Me] complexes was studied, since [Ni-Me] complexes are closer models for the growing [Ni-PE] polymeryl species in the insertion polymerization of ethylene than [Ni-Ph] complexes. Reaction of $1^{\mathrm{Me}}{ }_{\text {dmso }}$ with 2 equiv. of $\mathrm{PPh}_{3}$ and 7 equiv. of MMA resulted in the observed formation of 0.4 equiv. of $\mathrm{MePPh}_{3}{ }^{+}$and ethane $\left(\delta\left({ }^{1} \mathrm{H}\right)=0.82\right.$ (s) ppm, Scheme S 8, DMSO- $d_{6}, \mathrm{RT}, 10$ days, Figure S 52). No insertion of MMA into the [Ni-Me] bond or products thereof were observed, instead decomposition occurred. $\mathrm{MePPh}_{3}{ }^{+}$was also identified in the absence of MMA in the reaction of $\mathbf{1}^{\mathrm{Me}}{ }_{\mathrm{dmso}}$ with 4 equiv. of $\mathrm{PPh}_{3}$ by ${ }^{1} \mathrm{H}$ NMR spectroscopy (toluene- $d_{8}$ at $70{ }^{\circ} \mathrm{C}, 6 \mathrm{~h}$, Figure S 53).

The use of DMSO as a weakly coordinating ligand should facilitate a coordination of MMA to the [Ni] center and thereby the insertion into the Ni-C bond at less harsh conditions. Interestingly, even $\mathbf{1}^{\mathrm{Me}}$ dmso did not show any insertion of MMA or decomposition products thereof $\left(50{ }^{\circ} \mathrm{C}, 2\right.$ equiv. of MMA, DMSO- $d_{6}$, Figure S 54). Rather, formation of ethane due to bimolecular coupling was observed. The decline of the $\mathbf{1}^{\mathrm{Me}}{ }_{\mathrm{PPh} 3}$ resonance was monitored by ${ }^{31} \mathrm{P}$ NMR $\left(\delta\left({ }^{31} \mathrm{P}\left\{{ }^{1} \mathrm{H}\right\}\right)=28.7\right.$ (s) ppm) in the presence or the absence of MMA, showing the decomposition rate of [Ni-Me] to be independent of the MMA concentration (3 equiv. of $\mathrm{PPh}_{3}$, $70{ }^{\circ} \mathrm{C}$, toluene- $d_{8}$, Figure S 55). Berkefeld et al. ${ }^{35}$ previously reported that [Ni-Me] complexes show a significantly slower insertion than higher Ni-alkyl complexes such as [Ni-Et] for MA insertion into Ni-C bonds. However, even in the presence of ethylene (intermediately forming [Ni-Et] as a resting state) and MMA no insertion of MMA or products thereof could be observed 
under NMR experiment conditions. Instead, formation of $\mathrm{MePPh}_{3}{ }^{+}$was observed. In addition, pressure reactor experiments and analysis of the formed polymers revealed that the growing [Ni-PE] species is terminated by $\beta$-H elimination and that no insertion of MMA into higher [Ni-alkyl] complexes occurs (vide supra). NMR and pressure reactor experiments are in good agreement.

In conclusion, the [Ni-Me] complexes $\left(\mathbf{1}^{\mathrm{Me}} \mathrm{L}, \mathrm{L}=\mathrm{dmso}, \mathrm{PPh}_{3}\right)$, which are considered as model compounds for a growing [Ni-PE] species, do not insert MMA. Instead decomposition occurs, either by reductive elimination to the labile phosphine (where applicable, forming $\mathrm{MePPh}_{3}{ }^{+}$) or by bimolecular coupling (ethane formation). Both pathways are expected to form $[\mathrm{Ni}(0)]$ species.

Scheme S 9. Reactivity of [Ni-H] complex $\mathbf{1}^{\mathrm{H}}{ }_{\mathrm{PMe}}$ toward MMA at $-35^{\circ} \mathrm{C}$. The 1,2 insertion product $\mathbf{1}^{\mathrm{MMAH}_{\mathrm{PMe}}}$ is formed ( 2 equiv. of MMA, THF- $d_{8}$ ). At higher temperatures $\beta$-H elimination is favored.

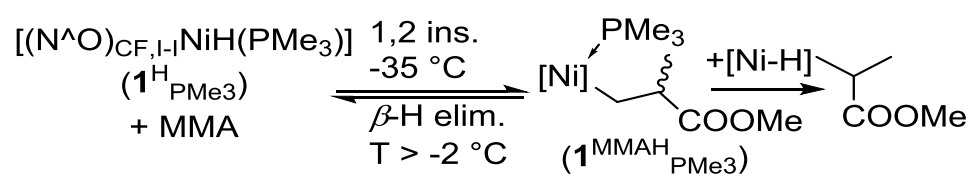

The aforementioned observations have shown that after ethylene insertion into catalyst precursors and after MMA insertion into [Ni-Ph] complexes $\beta$-H elimination can occur and intermediate reactive $[\mathrm{Ni}-\mathrm{H}]$ species are formed. To clarify the reactivity of such hydrides, MMA was reacted with the $[\mathrm{Ni}-\mathrm{H}]$ complex $\left[\left(\mathrm{N}^{\wedge} \mathrm{O}\right)_{\mathrm{CF}, \mathrm{I}-\mathrm{I}} \mathrm{NiH}\left(\mathrm{PMe}_{3}\right)\right]\left(\mathbf{1}^{\mathrm{H}}{ }_{\mathrm{PMe} 3}\right)$. An insertion in a 1,2-mode readily occurred at low temperatures $\left(-35{ }^{\circ} \mathrm{C}\right.$, Scheme S 9). The insertion product $\left[\left(\mathrm{N}^{\wedge} \mathrm{O}\right)_{\mathrm{CF}, \mathrm{II}} \mathrm{Ni}\left\{\mathrm{CH}_{2} \mathrm{C}\left(\mathrm{CH}_{3}\right) \mathrm{COOMe}\right\}\left(\mathrm{PMe}_{3}\right)\right] \quad\left(\mathbf{1}^{\left.\mathrm{MMAH}_{\mathrm{PMe} 3}\right)}\right.$ was sufficiently stable for characterization by NMR spectroscopy (Figure S 56, Figure S 57, Figure S 58). As a side product, methyl isobutyrate was observed, presumably via reaction of $\mathbf{1}^{\mathrm{H}_{\mathrm{PMe}}}$ with the [Ni-alkyl]

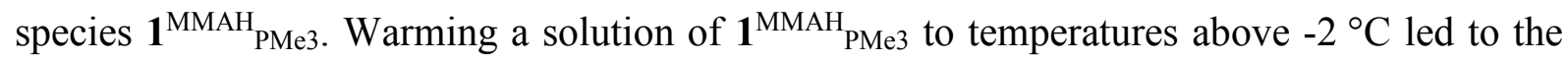
reverse reaction via $\beta$-H elimination, again forming the hydride $\mathbf{1}^{\mathrm{H}_{\mathrm{PMe}}}$ and MMA (Figure $\mathrm{S}$ 59).

Monitoring the insertion reaction over time by ${ }^{1} \mathrm{H}$ NMR spectroscopy (Figure S 60) yielded a second order rate constant for the 1,2 insertion of MMA into $[\mathrm{Ni}-\mathrm{H}]$ of $k_{\mathrm{obs}}=1.4 \pm 0.1 \cdot 10^{-3} \mathrm{M}^{-1} \mathrm{~s}^{-1}$ (Figure S 36). Berkefeld et al. reported a second order rate constant for the ethylene insertion into $1^{\mathrm{H}} \mathrm{PMe} 3$ of $1.7 \pm 0.1 \cdot 10^{-2} \mathrm{M}^{-1} \mathrm{~s}^{-1}$ at $-9{ }^{\circ} \mathrm{C} .{ }^{35}$ Considering the difference in temperature for the derived rates $\left(-35^{\circ} \mathrm{C}\right.$ and $-9{ }^{\circ} \mathrm{C}$ for $\mathrm{MMA}$ and ethylene, respectively), one could roughly estimate that the rate of insertion for ethylene and MMA into $\mathbf{1}^{\mathrm{H}_{\mathrm{PMe} 3}}$ is on a similar order. Therefore, the reversible insertion of MMA into intermediately formed [Ni-H] species during the simultaneous polymerization of ethylene and MMA seems feasible. 
a)

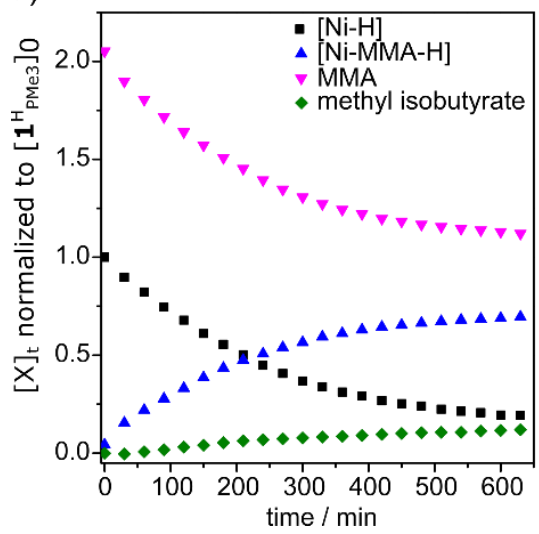

b)

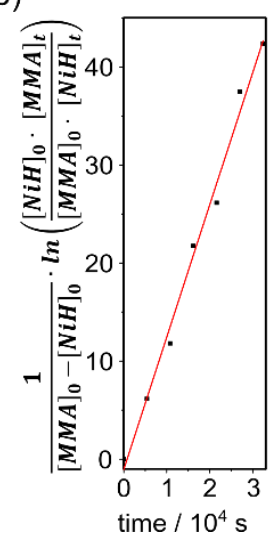

Figure S 36. Reaction monitoring of the insertion of MMA (2 equiv.) into $\mathbf{1}^{\mathrm{H}_{\mathrm{PMe}}}$ at $-35^{\circ} \mathrm{C}$ in THF- $d_{8}$ via ${ }^{1} \mathrm{H}$ NMR spectroscopy. (a) Time dependent relative concentrations of [Ni-H], [Ni-MMAH], MMA and methyl isobutyrate normalized to $[\mathrm{Ni}-\mathrm{H}]_{0}$. (b) Plot of the determination of the second-order rate constant for the insertion of MMA into $[\mathrm{Ni}-\mathrm{H}]\left(k_{\mathrm{obs}}=1.35 \pm 0.06 \cdot 10^{-3} \mathrm{M}^{-1} \mathrm{~s}^{-1}\right)$.

Interestingly, the 1,2 insertion mode of MMA is in contrast to the 2,1 insertion mode of MA

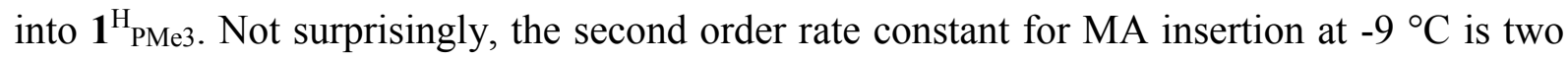
orders of magnitude higher $\left(2.1 \pm 0.1 \cdot 10^{-1} \mathrm{M}^{-1} \mathrm{~s}^{-1}\right)$ than for an insertion of the sterically more demanding MMA. Vinyl acetate was found to also insert in a 1,2 mode into $\mathbf{1}^{\mathrm{H}_{\mathrm{PMe}}}$, which is described as the kinetic insertion product and rearranges over time to the thermodynamic more favored 2,1 product. In analogy, the sterically demanding MMA could favor a transition state which leads to the kinetic 1,2 insertion product in contrast to MA. ${ }^{35}$

A similar reactivity was observed for phosphinesulfonato $\mathrm{Pd}(\mathrm{II})$ methyl complexes. MMA inserts in a 1,2 and 2,1 mode in an overall ratio 3:2. Intermediate $\left[\left(\mathrm{P}^{\wedge} \mathrm{O}\right) \mathrm{PdH}\right]$ fragments, formed after $\beta$-H elimination from alkyl complexes, also prefer a 1,2 insertion over the 2,1 mode for MMA. In contrast, MA was found to insert exclusively in the 2,1 mode into [ $\left.\left(\mathrm{P}^{\wedge} \mathrm{O}\right) \mathrm{PdMe}\right]$. An overall kinetic preference for the MA insertion over the MMA insertion with a factor of $\sim 10^{2}$ was estimated. ${ }^{36}$ 


\subsection{Additional spectroscopic data}

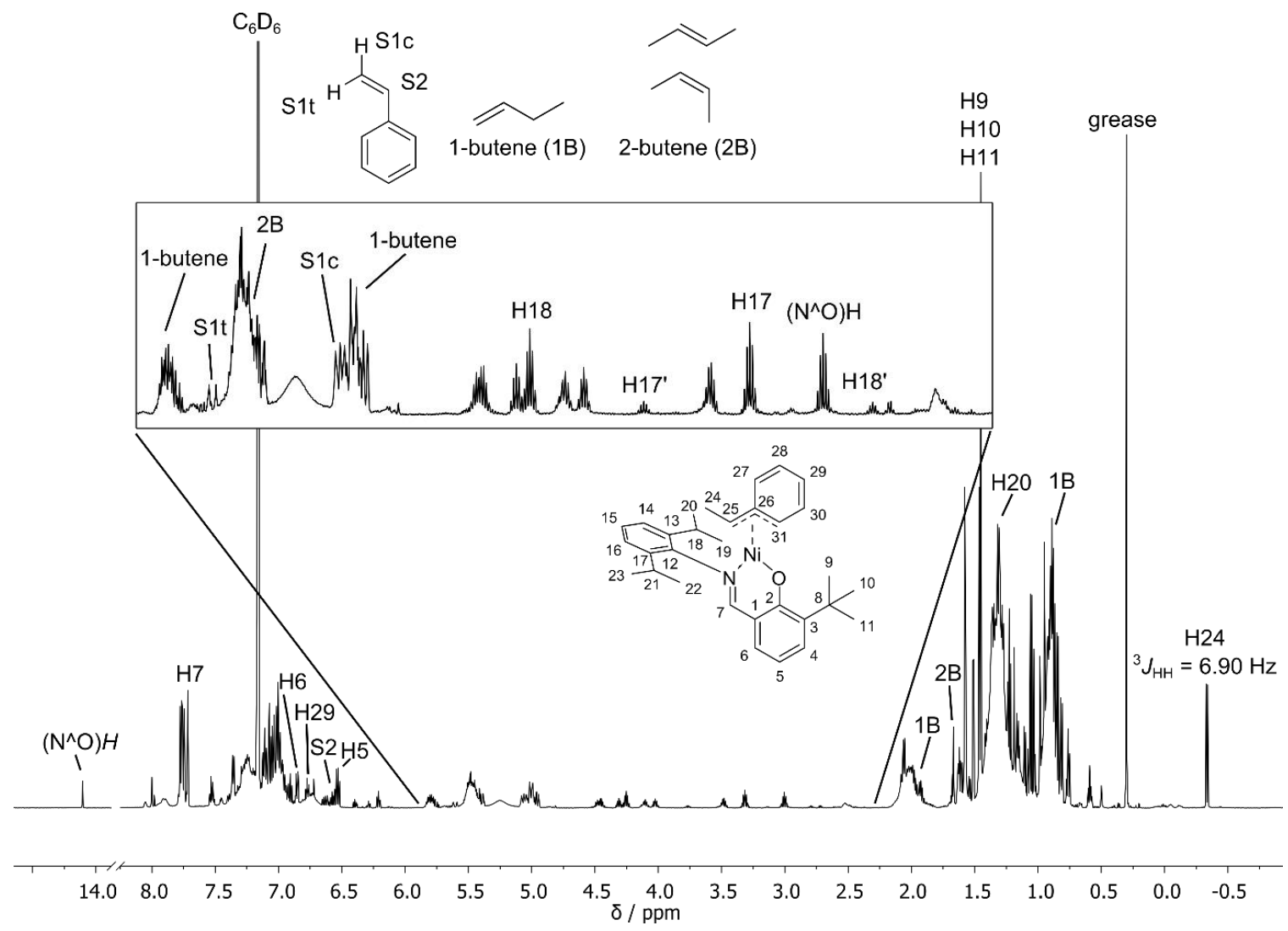

Figure S 37. ${ }^{1} \mathrm{H}$ NMR $\left(600 \mathrm{MHz}, \mathrm{C}_{6} \mathrm{D}_{6}, \mathrm{RT}\right)$ spectrum of the reaction mixture of $3^{\mathrm{Ph}}{ }_{\mathrm{PPh}}$ and $5 \times 3$ equiv. of ethylene after $72 \mathrm{~h}$ at $6{ }^{\circ} \mathrm{C}$. The NMR sample was filtered through a syringe filter to separate PE formed. The insertion of ethylene and isomerization leads to the formation of the $\eta^{3}$-benzyl $\mathrm{Ni}$ (II) complex which is shown as inset. In addition, styrene (S1t, S1c, S2) was observed. This $\eta^{3}$-benzyl Ni complex is in equilibrium with a second isomer (exchange resonances in ${ }^{1} \mathrm{H}-{ }^{1} \mathrm{H}$ NOESY, methine protons labeled with $\mathrm{H} 17$ ' and $\mathrm{H} 18$ ', Figure S 38). In addition, the formation of butenes (1-butene and 2-butenes) was observed from dimerization starting from [Ni-H]. Reductive elimination from $[\mathrm{Ni}-\mathrm{H}]$ to the $\left(\mathrm{N}^{\wedge} \mathrm{O}\right)^{-}$ligand led to formation of $\left(\mathrm{N}^{\wedge} \mathrm{O}\right) \mathrm{H}\left(14.1 \mathrm{ppm},-\mathrm{CH}\left(\mathrm{CH}_{3}\right)_{2} 3.00 \mathrm{ppm}\right)$.

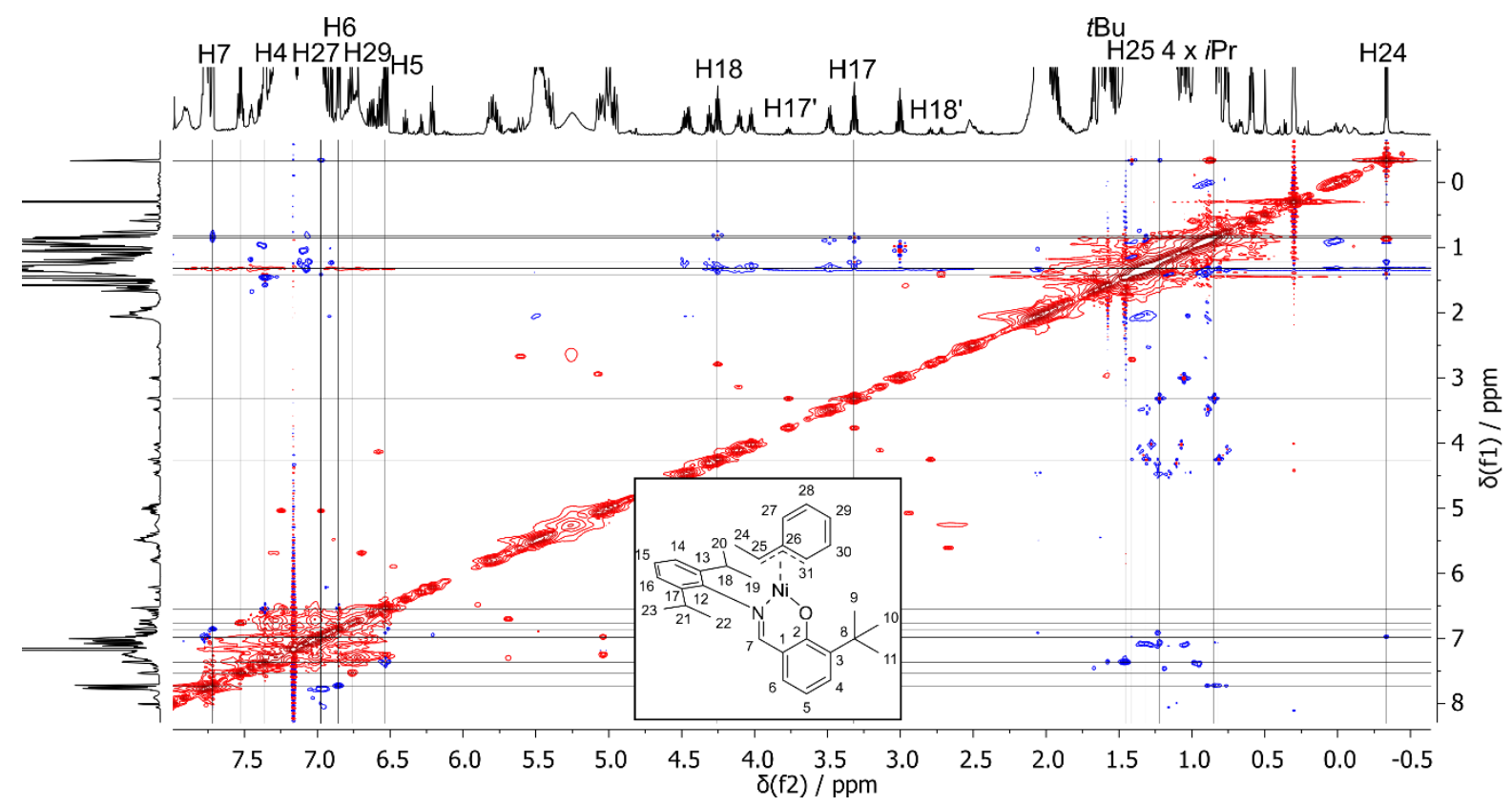

Figure S 38. ${ }^{1} \mathrm{H}-{ }^{1} \mathrm{H}$ NOESY $\left(600 \mathrm{MHz}, \mathrm{C}_{6} \mathrm{D}_{6}, \mathrm{RT}\right)$ of the reaction mixture of $\mathbf{3}^{\mathrm{Ph}}{ }_{\mathrm{PPh} 3}$ and $5 \times 3$ equiv. ethylene after $72 \mathrm{~h}$ at $6{ }^{\circ} \mathrm{C}$. The NMR sample was filtered with a syringe filter to separate PE formed. 
$\mathrm{t}=48 \mathrm{~h}$

$3 \times 3$ eq. ethylene

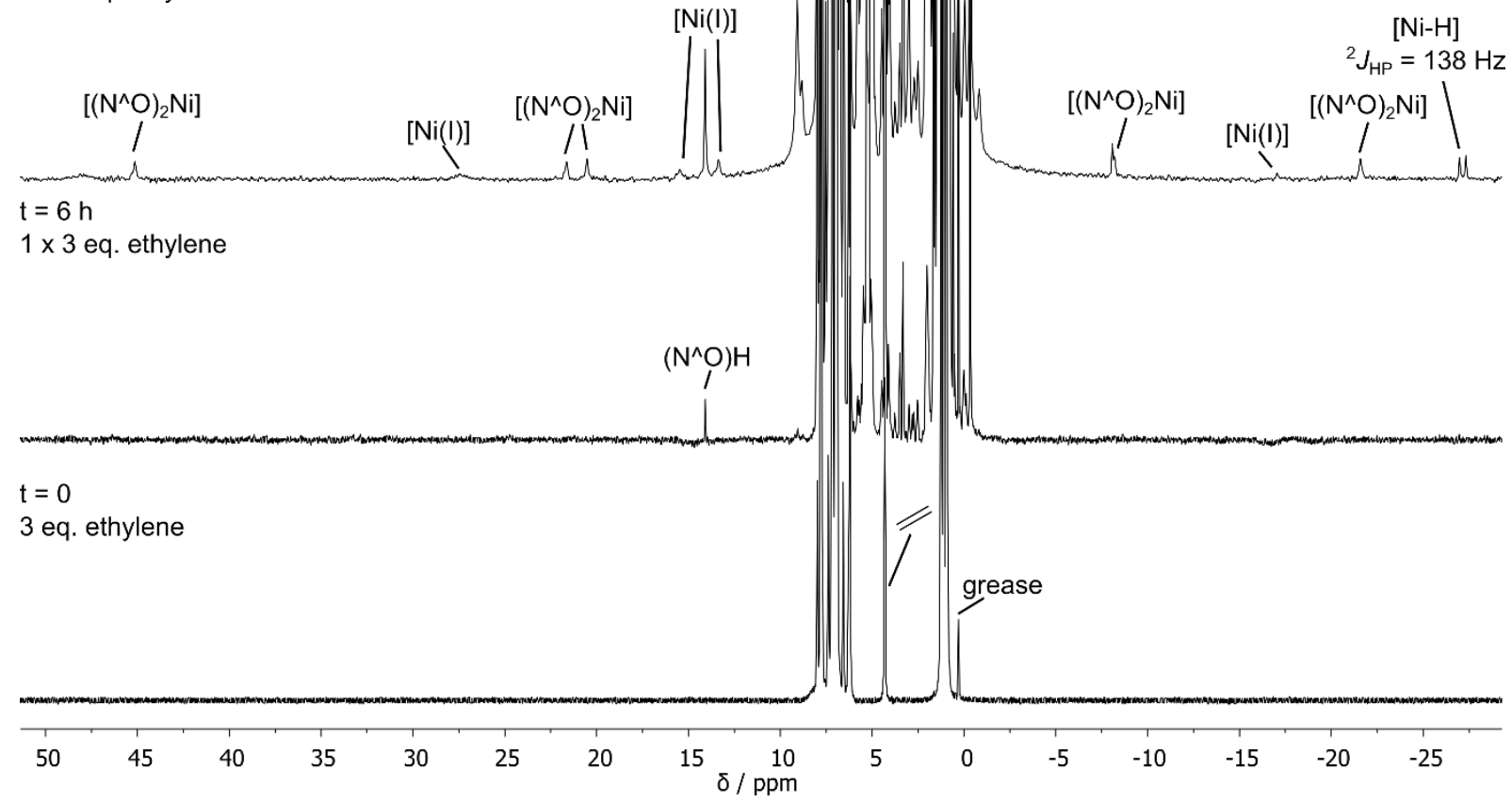

Figure S 39. ${ }^{1} \mathrm{H}$ NMR $\left(400 \mathrm{MHz}, \mathrm{C}_{6} \mathrm{D}_{6}, \mathrm{RT}\right)$ reaction monitoring of a mixture of $\mathbf{3}^{\mathrm{Ph}}{ }_{\mathrm{PPh}}(27 \mathrm{mM})$ and incremental added ethylene at room temperature after $0 \mathrm{~h}$ (bottom), $6 \mathrm{~h}$ (middle) and $48 \mathrm{~h}$ (top, $20 \mathrm{~Hz}$ Gaussian line broadening). A [Ni-H] species, formed after $\beta$-H elimination from [Ni-alkyl] complexes, intermediate $[\mathrm{Ni}(\mathrm{I})]$ species and bis-chelated complex $\left[\left(\mathrm{N}^{\wedge} \mathrm{O}\right)_{2} \mathrm{Ni}\right]$ were observed.

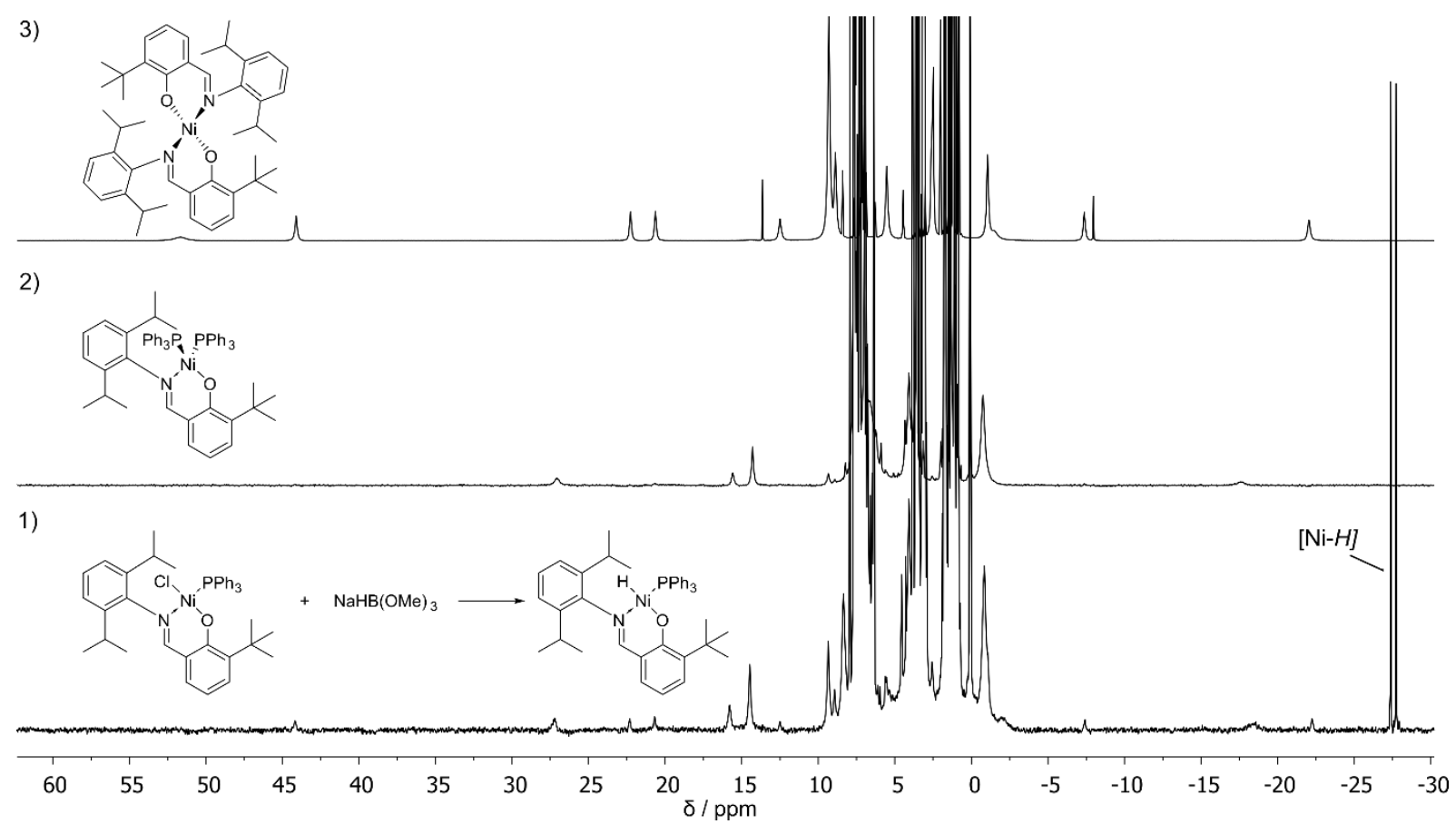

Figure S 40. ${ }^{1} \mathrm{H}$ NMR spectra (400 MHz, THF- $\left.d_{8}, \mathrm{RT}\right)$ of $3^{{ }^{\mathrm{Cl}}}{ }_{\mathrm{PPh} 3}(26 \mathrm{mM})$ with 3 equiv. $\mathrm{NaHB}(\mathrm{OMe})_{3}$ forming $3^{\mathrm{H}}{ }_{\mathrm{PPh} 3}$ after $22 \mathrm{~h}$ at room temperature (1). Two sets of anisotropic shifted resonances appeared during reaction. By comparison with separately synthesized complexes, they were assigned to the corresponding $[\mathrm{Ni}(\mathrm{I})]$ complex $\left(3^{\mathrm{PPh} 3}{ }_{\mathrm{PPh}}, 2\right)$ and the bis-chelated complex $\{\mathbf{3 - 3}\}$ (3). 


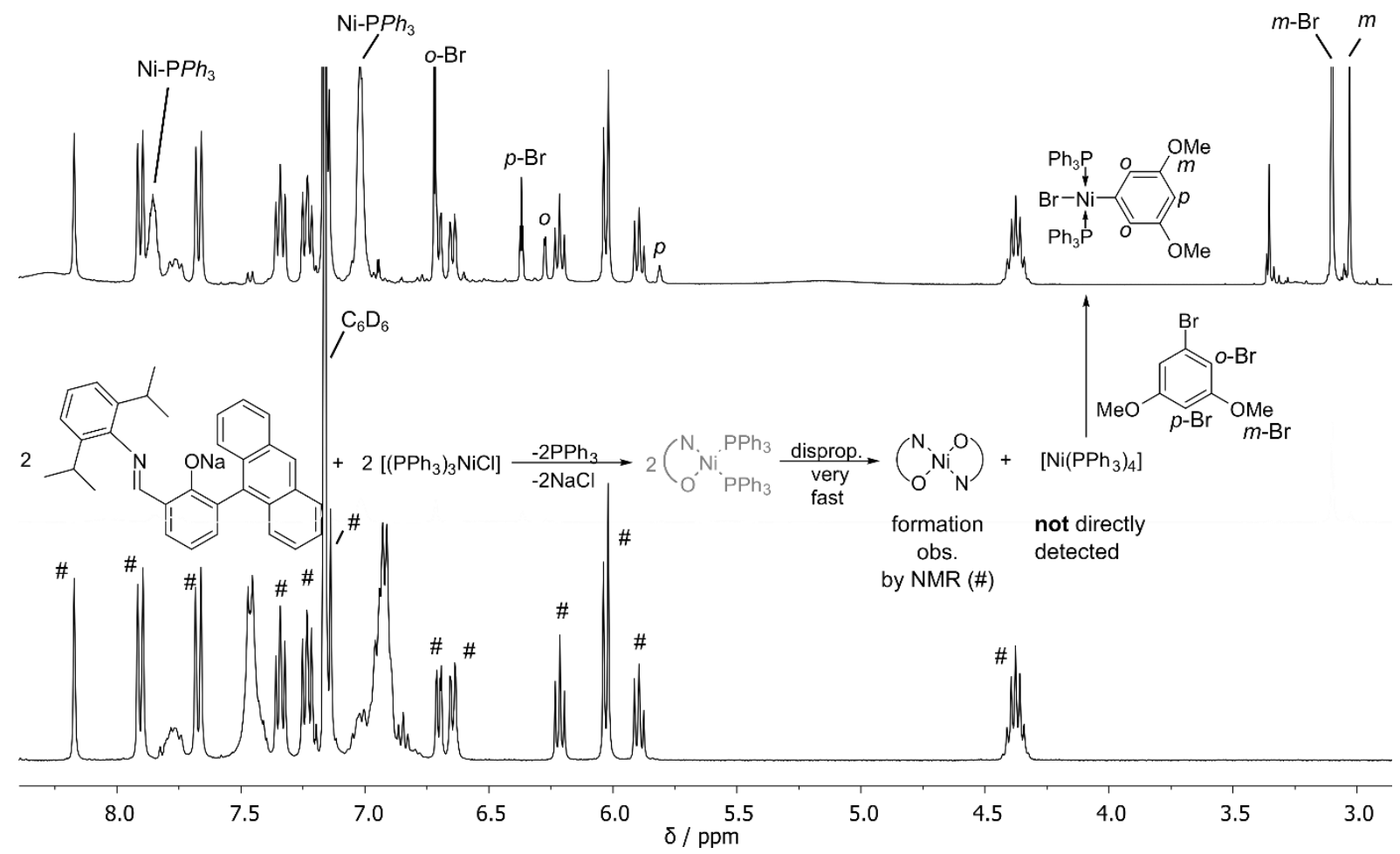

Figure $\mathrm{S} 41$. Indirect observation of $\mathrm{Ni}(0)$-species formed by disproportionation resulting from intermediate $\mathrm{Ni}(\mathrm{I})$ species. Lower spectrum $\left({ }^{1} \mathrm{H}\right.$ NMR, $\left.400 \mathrm{MHz}, \mathrm{C}_{6} \mathrm{D}_{6}, \mathrm{RT}\right)$ shows the reaction mixture of $\left(\mathrm{N}^{\wedge} \mathrm{O}\right)_{i \mathrm{Pr}, \mathrm{Ant}-\mathrm{H}} \mathrm{Na}$ with $\left[\left(\mathrm{PPh}_{3}\right)_{3} \mathrm{Ni}(\mathrm{I}) \mathrm{Cl}\right]$. Intermediately formed $\left(\mathrm{N}^{\wedge} \mathrm{O}\right) \mathrm{Ni}(\mathrm{I})$ complex underwent very rapid disproportionation at room temperature and bis-chelated complex $\{\mathbf{4 - 4}\}$ was observed. The corresponding Ni(0)-species could not be unambiguously assigned by NMR spectroscopy and was indirectly detected by oxidative addition reaction with 3,5-dimethoxybromobenzene (upper spectrum) forming trans-bromo(3,5dimethoxyphenyl)bis(triphenylphosphine)nickel(II) which was identified by ${ }^{1} \mathrm{H}$ NMR spectroscopy in solution.

Reaction of $\left[\left(N^{\wedge} O\right) N i(I I) R(L)\right]$ catalyst precursors and intermediate Ni-species with MMA.

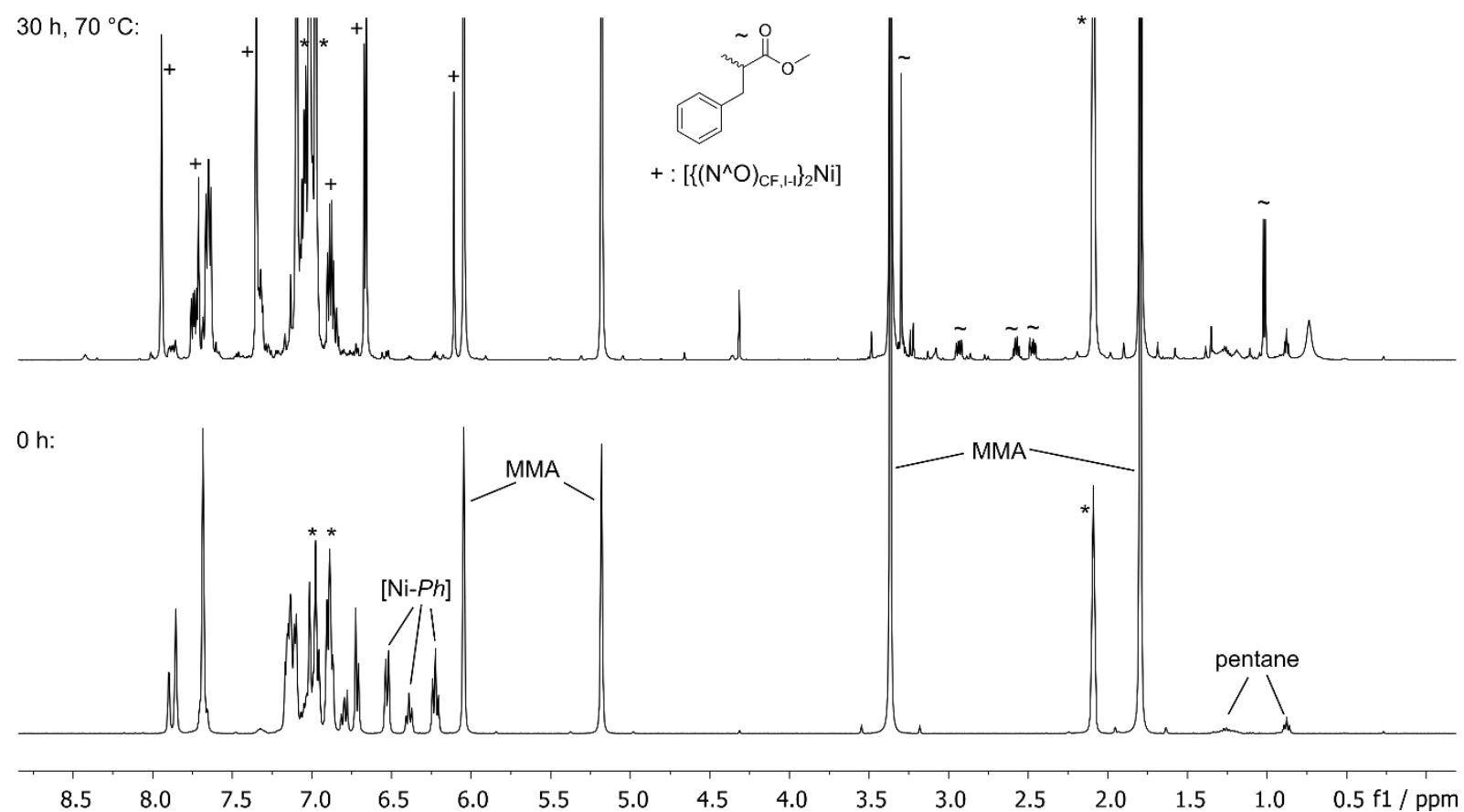

Figure S 42. ${ }^{1} \mathrm{H}$ NMR spectra ( $0 \mathrm{~h}: 400 \mathrm{MHz} ; 30 \mathrm{~h}: 600 \mathrm{MHz}$, toluene- $d_{8}$, RT) of the reaction mixture of $\mathbf{1}^{\mathrm{Ph}}{ }_{\mathrm{PPh} 3}$ $(15 \mathrm{mM})$ with 5 equiv. of MMA after $0 \mathrm{~h}$ and $30 \mathrm{~h}$ at $70{ }^{\circ} \mathrm{C}$. In the upper spectrum, characteristic resonances of methyl 2-methyl-3-phenylpropanoate $(\sim)$ and bis-chelated complex $\{\mathbf{1 - 1}\}(+)$ are labeled. 


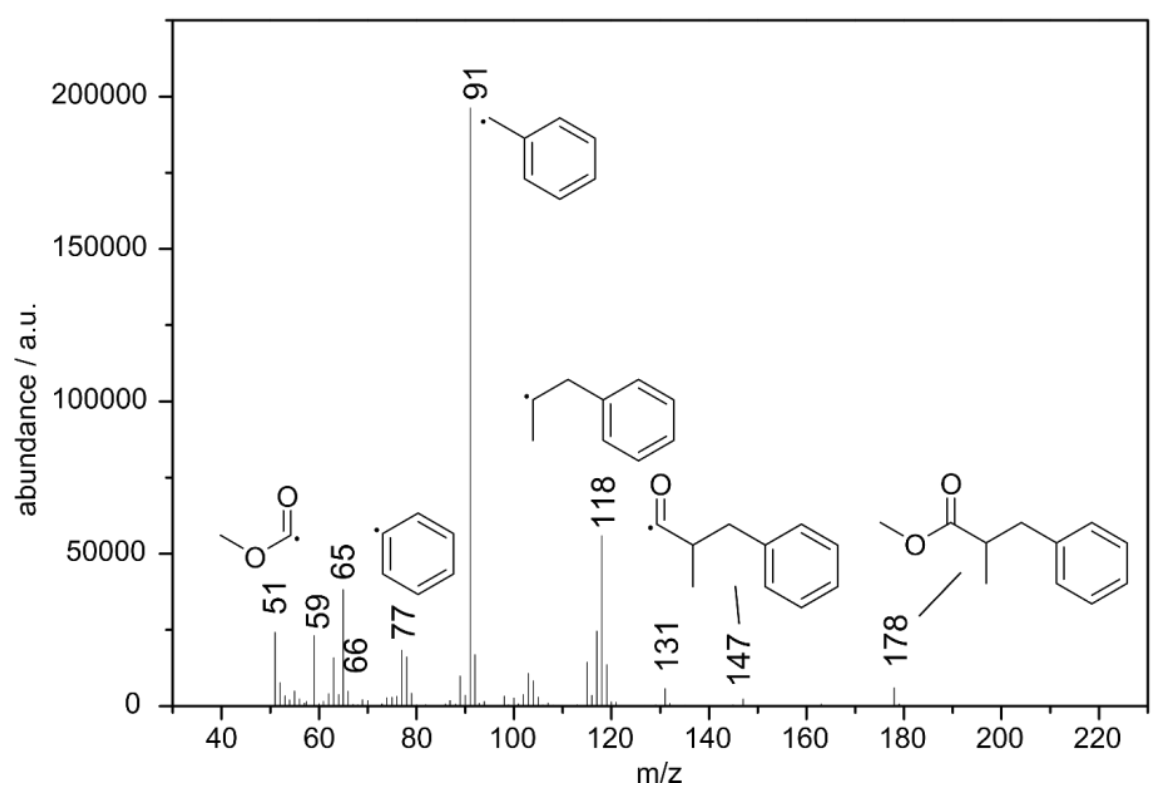

Figure S 43. GC/MS of the reaction mixture of $\mathbf{1}^{\mathrm{Ph}}{ }_{\mathrm{PPh} 3}(15 \mathrm{mM})$ and 5 equiv. of MMA after $30 \mathrm{~h}$ at $70{ }^{\circ} \mathrm{C}$. The inset shows the assignment of the fragmentation pattern and the mass peak of the saturated organic product (methyl 2-methyl-3-phenylpropanoate) after 2,1 insertion of MMA into $\mathbf{1}^{\mathrm{Ph}}{ }_{\mathrm{PPh} 3}$ and subsequent reaction with a [Ni-H] species.

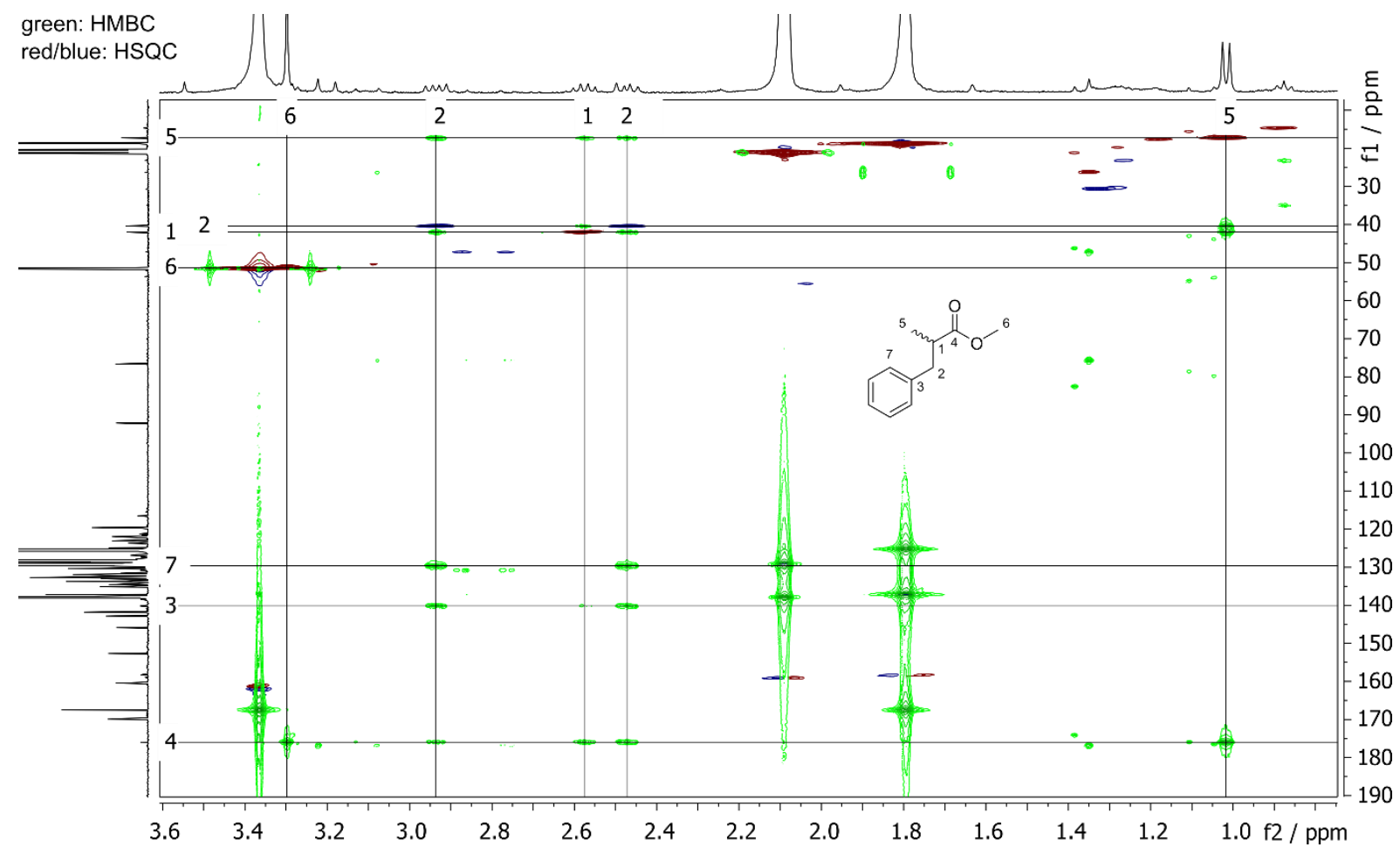

Figure S 44. Superimposed ${ }^{1} \mathrm{H}_{-}{ }^{13} \mathrm{C}$ HMBC (green) and ${ }^{1} \mathrm{H}_{-13}{ }^{13} \mathrm{HSQC}$ (red/blue) NMR spectra $(600 \mathrm{MHz}$, toluene- $\left.d_{8}, \mathrm{RT}\right)$ of the reaction mixture of $\mathbf{1}^{\mathrm{Ph}} \mathrm{PPh} 3(15 \mathrm{mM})$ and 5 equiv. of MMA after $30 \mathrm{~h}$ at $70^{\circ} \mathrm{C}$. The inset shows the assignment of the saturated organic product (methyl 2-methyl-3-phenylpropionate) after 2,1 insertion of MMA into $\mathbf{1}^{\mathrm{Ph}}{ }_{\mathrm{PPh} 3}$ and subsequent reaction with a $[\mathrm{Ni}-\mathrm{H}]$ species. 


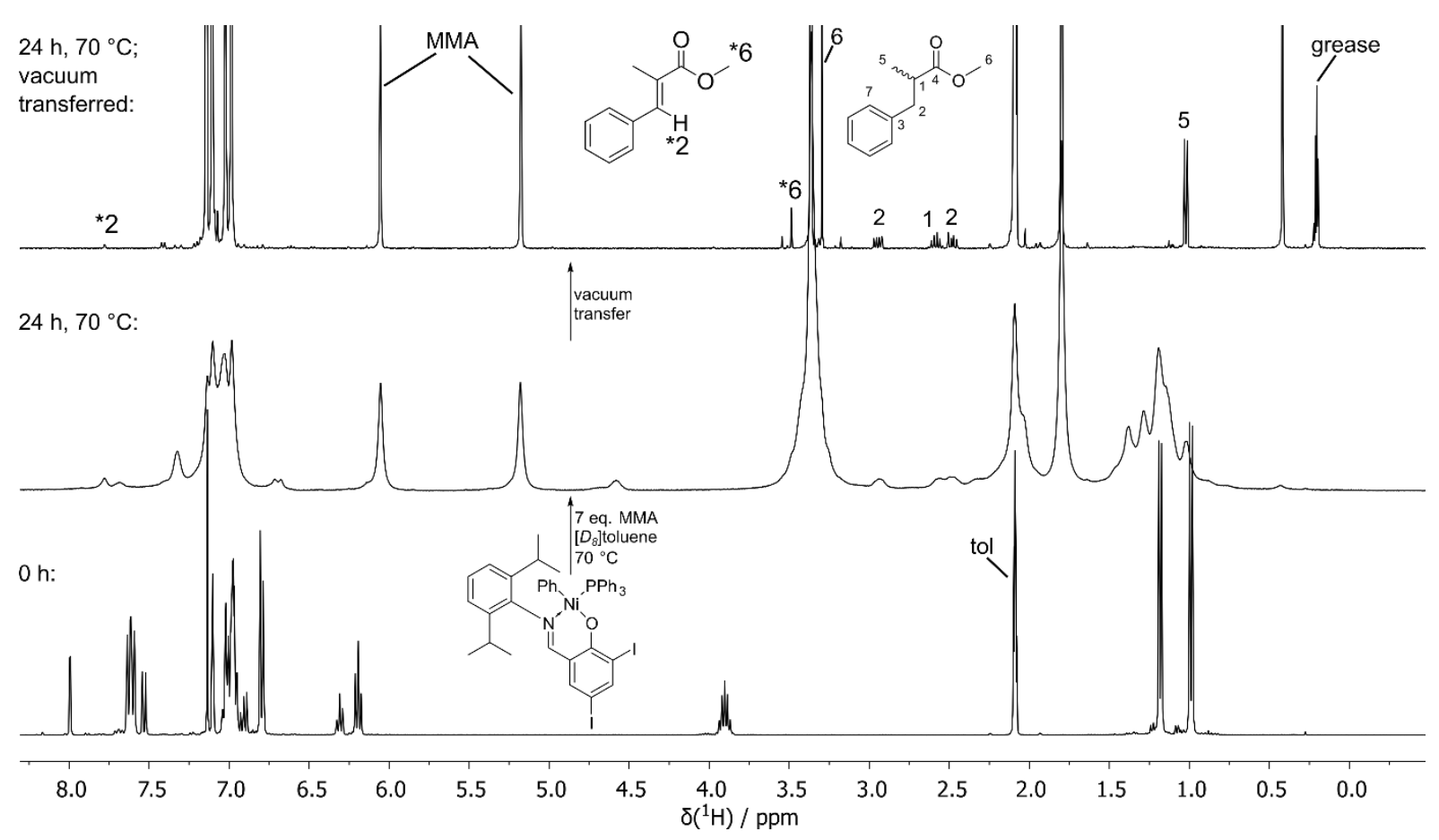

Figure S 45. ${ }^{1} \mathrm{H}$ NMR spectra $\left(400 \mathrm{MHz}\right.$, toluene- $d_{8}$, RT) of $2^{\mathrm{Ph}}{ }_{\mathrm{PPh} 3}(15 \mathrm{mM})$ before addition $(0 \mathrm{~h})$ of 7 equiv. of MMA and heating for $24 \mathrm{~h}$ at $70{ }^{\circ} \mathrm{C}$. Due to a significant peak broadening and PMMA formation an identification of the reaction products was difficult and the reaction mixture was separated by vacuum transfer in a low and high boiling fraction. The upper spectrum shows the low boiling fraction and characteristic resonances of saturated organic product after 2,1 ins. of MMA into [Ni-Ph] and reaction with [Ni-H]. In addition, methyl 2-methyl- $(E)-3-$ phenyl-2-propenoate was formed after 2,1 insertion of MMA followed by $\beta$-H elimination (*).

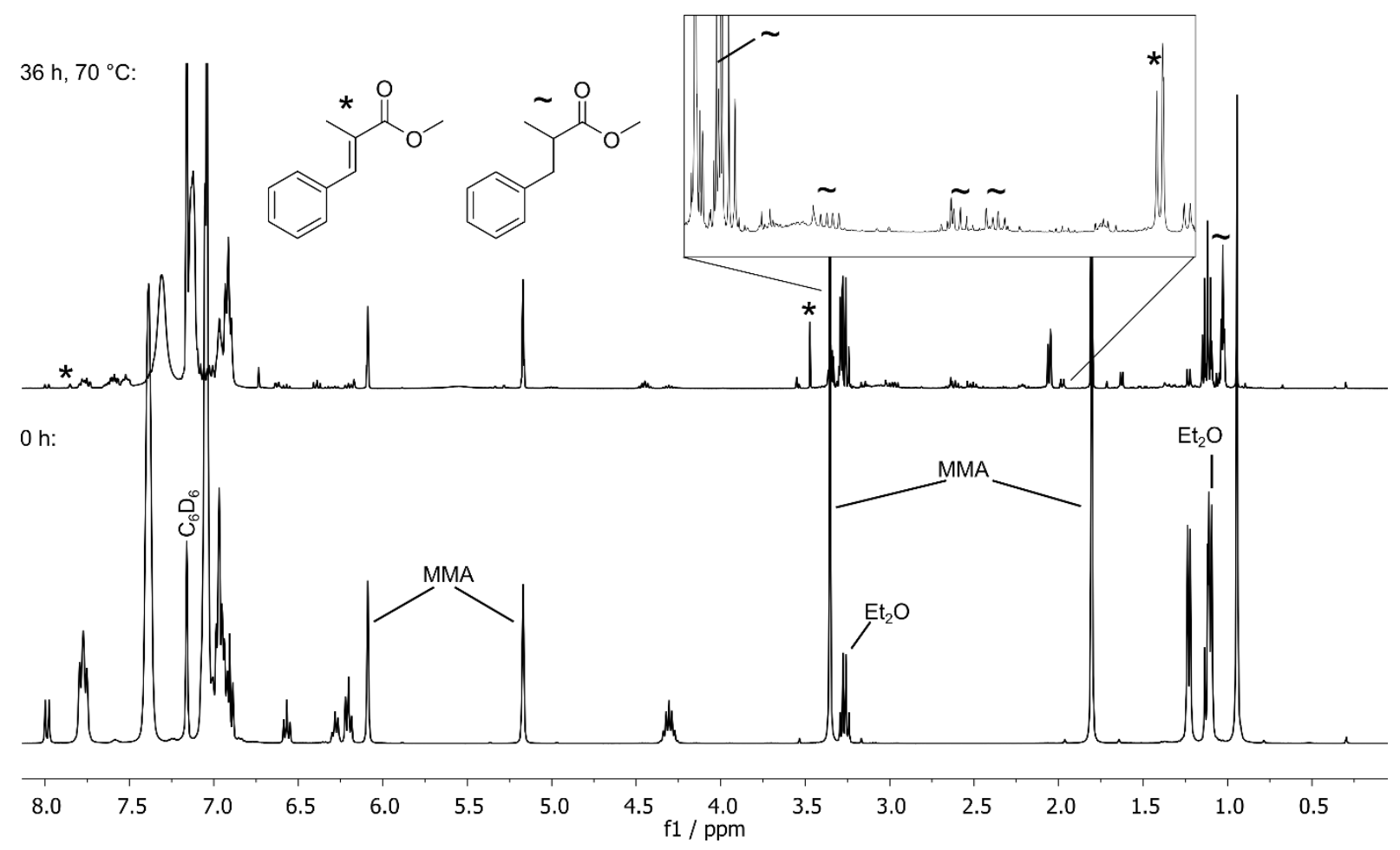

Figure S 46. ${ }^{1} \mathrm{H}$ NMR spectra (400 MHz, $\left.\mathrm{C}_{6} \mathrm{D}_{6}, \mathrm{RT}\right)$ of the reaction mixture of $3^{\mathrm{Ph}}{ }_{\mathrm{PPh} 3}(14 \mathrm{mM}), 3$ equiv. of MMA and 3 equiv. of $\mathrm{PPh}_{3}$ after $0 \mathrm{~min}$ and $36 \mathrm{~h}$ at $70{ }^{\circ} \mathrm{C}$. All resonances which are not assigned in the spectrum after 0 $\mathrm{h}$ are resulting from $3^{\mathrm{Ph}}{ }_{\mathrm{PPh} 3}$ or $\mathrm{PPh}_{3}$. In the upper spectrum characteristic saturated ( , reaction of 2,1 insertion product with $[\mathrm{Ni}-\mathrm{H}])$ and unsaturated $(*)$ organic product after 2,1 ins. of MMA into [Ni-Ph] and $\beta$-H elimination are labeled. 


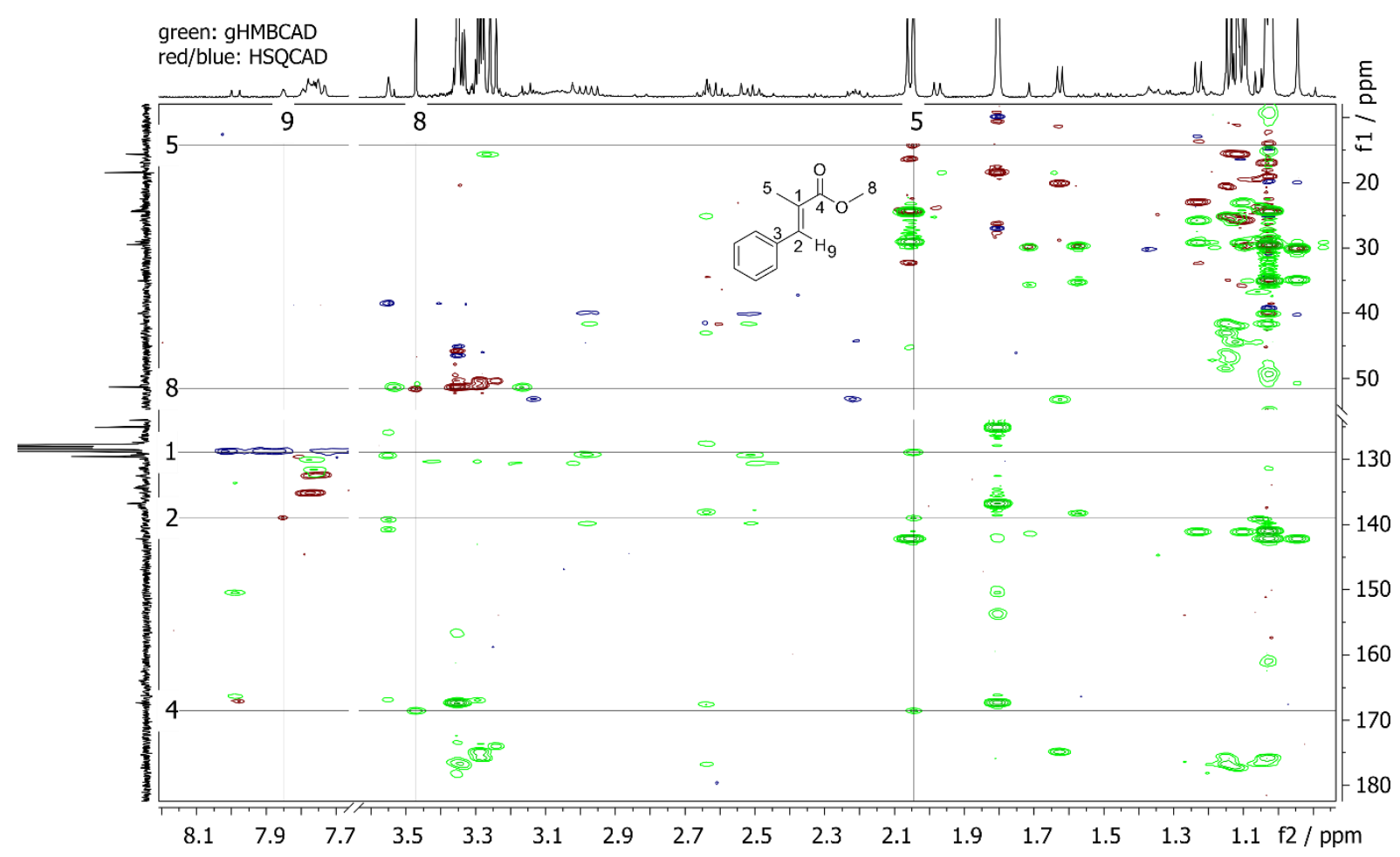

Figure S 47. Superimposed ${ }^{1} \mathrm{H}-{ }^{13} \mathrm{C}$ gHMBCAD (green) and ${ }^{1} \mathrm{H}-{ }^{13} \mathrm{C}$ HSQCAD (red/blue) NMR spectra $(400 \mathrm{MHz}$, $\left.\mathrm{C}_{6} \mathrm{D}_{6}, 25^{\circ} \mathrm{C}\right)$ of the reaction mixture of $3^{\mathrm{Ph}}{ }_{\mathrm{PPh} 3}(14 \mathrm{mM}), 3$ equiv. of MMA and 3 equiv. of $\mathrm{PPh}_{3}$ after $36 \mathrm{~h}$ at $70{ }^{\circ} \mathrm{C}$. The inset shows the assignment of the unsaturated $\beta$-H elimination product (methyl 2-methyl- $(E)-3$-phenyl-2propenoate) after 2,1 insertion of MMA into $3^{\mathrm{Ph}}{ }_{\mathrm{PPh}}$. The assignment is in agreement with reported spectroscopic features of methyl 2-methyl-(E)-3-phenyl-2-propenoate. ${ }^{37}$

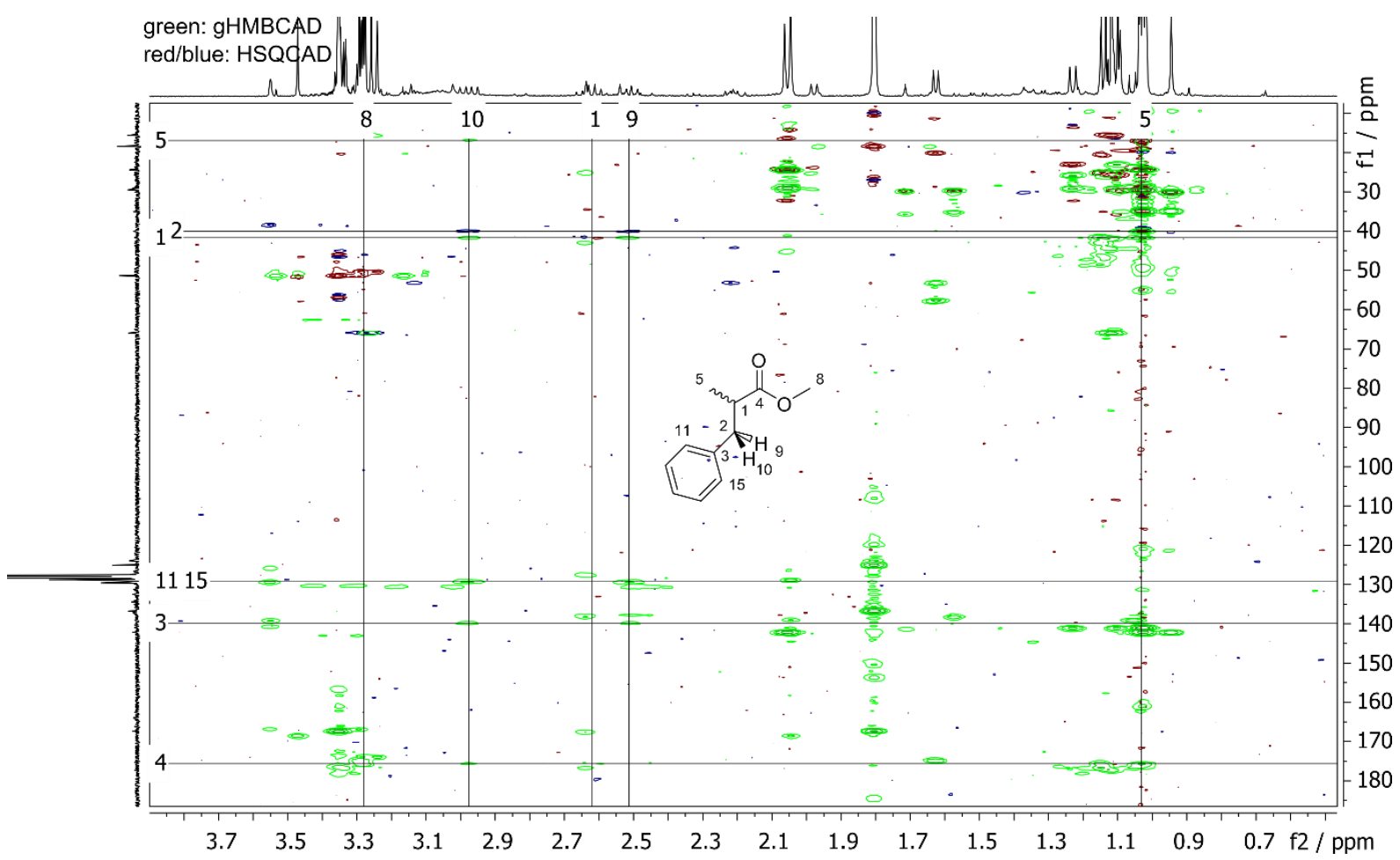

Figure S 48. Superimposed ${ }^{1} \mathrm{H}-{ }^{13} \mathrm{C}$ gHMBCAD (green) and ${ }^{1} \mathrm{H}^{-13} \mathrm{C}$ HSQCAD (red/blue) NMR spectra $(400 \mathrm{MHz}$, $\left.\mathrm{RT}, \mathrm{C}_{6} \mathrm{D}_{6}\right)$ of the reaction mixture of $3^{\mathrm{Ph}}{ }_{\mathrm{PPh} 3}(14 \mathrm{mM}), 3$ equiv. of MMA and 3 equiv. of $\mathrm{PPh}_{3}$ after $36 \mathrm{~h}$ at $70{ }^{\circ} \mathrm{C}$. The inset shows the assignment of the saturated organic product (methyl 2-methyl-3-phenylpropanoate) after 2,1 insertion of MMA into $3^{\mathrm{Ph}}$ PPh3 and subsequent reaction with a [Ni-H] species. 


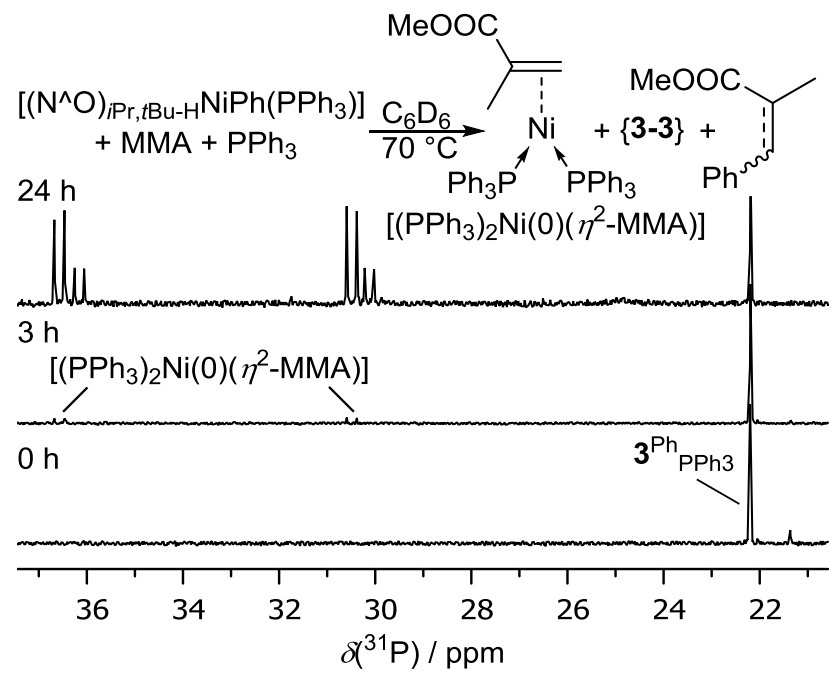

Figure S 49. ${ }^{31} \mathrm{P}\left\{{ }^{1} \mathrm{H}\right\} \mathrm{NMR}\left(162 \mathrm{MHz}, \mathrm{C}_{6} \mathrm{D}_{6}, \mathrm{RT}\right)$ spectroscopic reaction monitoring of the reaction of $3^{\mathrm{Ph}}{ }_{\mathrm{PPh} 3}$ with 3 equiv. of MMA and 3 equiv. of $\mathrm{PPh}_{3}$ at $70{ }^{\circ} \mathrm{C}$ after $0 \mathrm{~h}, 3 \mathrm{~h}$ and $24 \mathrm{~h}$, respectively. Beside organic products (vide supra), bis-chelated $\mathrm{Ni}(\mathrm{II})$ complex and $\left[\left(\mathrm{PPh}_{3}\right)_{2} \mathrm{Ni}(0)\left(\eta^{2}-\mathrm{H}_{2} \mathrm{C}=\mathrm{C}\left(\mathrm{CH}_{3}\right) \mathrm{COOMe}\right)\right]$ were identified. The two $\mathrm{Ni}$ complexes were presumably formed via reductive coupling and subsequent disproportionation of the intermediately formed $[\mathrm{Ni}(\mathrm{I})]$ complex.

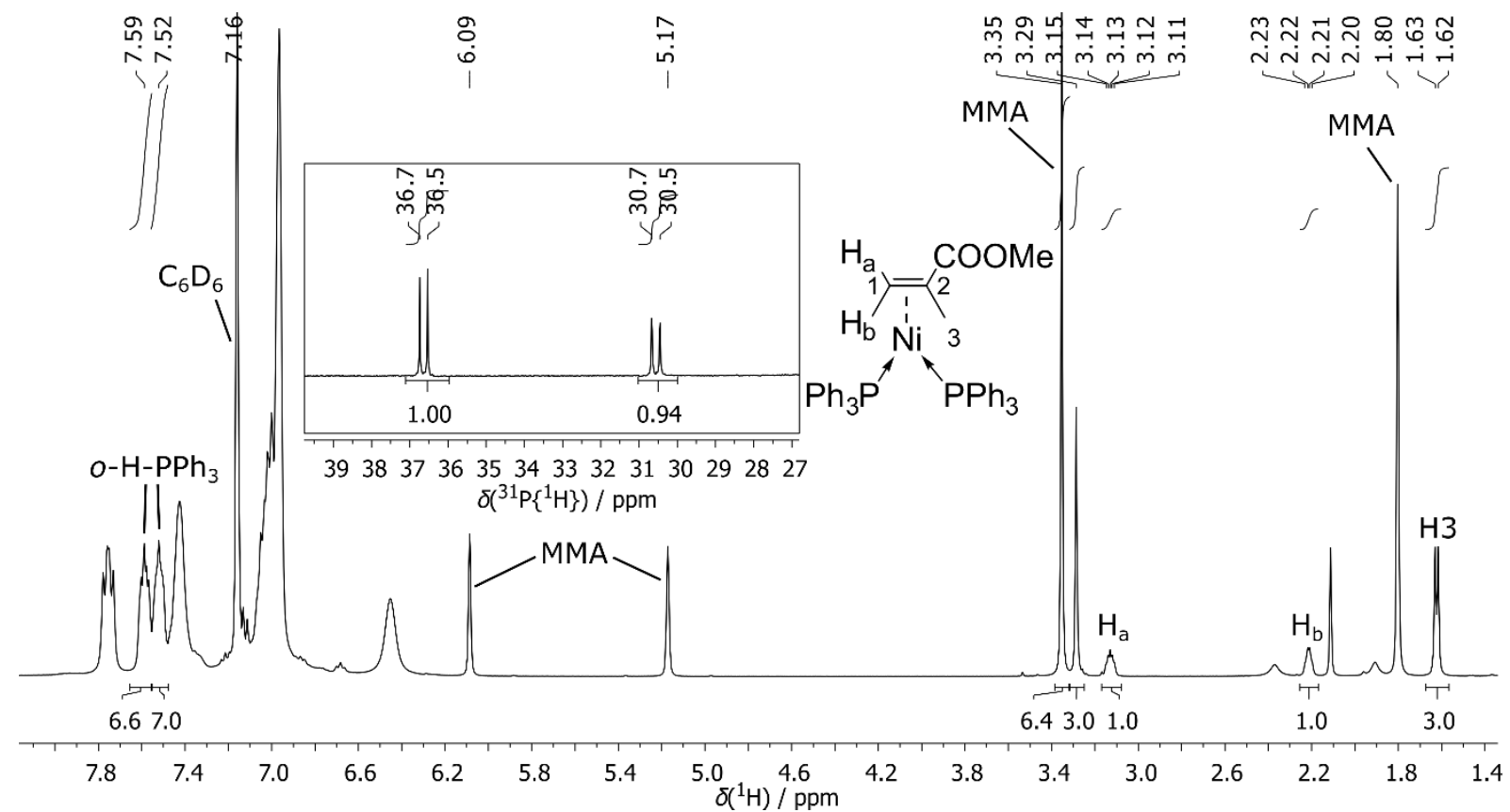

Figure S 50. ${ }^{1} \mathrm{H}$ NMR spectrum $\left(400 \mathrm{MHz}, \mathrm{C}_{6} \mathrm{D}_{6}, \mathrm{RT}\right)$ showing the reaction of $\left[\mathrm{Ni}(0)\left(\mathrm{PPh}_{3}\right)_{4}\right]$ with 2 equiv. of MMA at RT after 10 minutes. The coordination compound $\left[\left(\mathrm{PPh}_{3}\right)_{2} \mathrm{Ni}\left(\eta^{2}-\mathrm{H} 2 \mathrm{C}=\mathrm{C}\left(\mathrm{CH}_{3}\right) \mathrm{COOMe}\right)\right]$ was formed and the assignment is given as inset. The corresponding ${ }^{31} \mathrm{P}\left\{{ }^{1} \mathrm{H}\right\} \mathrm{NMR}\left(162 \mathrm{MHz}, \mathrm{C}_{6} \mathrm{D}_{6}, \mathrm{RT}\right)$ spectrum is given as inset.

Characteristic resonances of $\left[\left(\mathrm{PPh}_{3}\right)_{2} \mathrm{Ni}\left(\eta^{2}-\mathrm{H}_{2} \mathrm{C}=\mathrm{C}\left(\mathrm{CH}_{3}\right) \mathrm{COOMe}\right)\right]$ :

${ }^{1} \mathrm{H}$ NMR (400 MHz, $\left.\mathrm{C}_{6} \mathrm{D}_{6}, \mathrm{RT}\right): \delta=7.59$ (m, 6H, o- $\left.\mathrm{H}-\mathrm{P}^{1} \mathrm{Ph}_{3}\right), 7.52\left(\mathrm{~m}, 6 \mathrm{H}, o-\mathrm{H}-\mathrm{P}^{2} \mathrm{Ph}_{3}\right.$ ), 3.29 (s, $\left.3 \mathrm{H},-\mathrm{COOCH}_{3}\right), 3.13\left(\mathrm{~m}, 1 \mathrm{H}, \mathrm{H}_{\mathrm{a}}\right), 2.21\left(\mathrm{~m}, 1 \mathrm{H}, \mathrm{H}_{\mathrm{b}}\right), 1.62\left(\mathrm{~d},{ }^{3} J_{H P}=5.6 \mathrm{~Hz}, 3 \mathrm{H}, \mathrm{H} 3\right)$.

${ }^{13} \mathrm{C}\left\{{ }^{1} \mathrm{H}\right\}$ NMR $\left(101 \mathrm{MHz}, \mathrm{C}_{6} \mathrm{D}_{6}, \mathrm{RT}\right): \delta=174.9(-\mathrm{COOMe}), 136.8\left(\mathrm{dd},{ }^{1} J_{\mathrm{CP}}=54.8 \mathrm{~Hz},{ }^{3} J_{\mathrm{CP}}=\right.$ $\left.2.8 \mathrm{~Hz}, i-C-\mathrm{P}^{1} \mathrm{Ph}_{3}\right), 136.4\left(\mathrm{dd},{ }^{1} J_{\mathrm{CP}}=54.2 \mathrm{~Hz},{ }^{3} J_{\mathrm{CP}}=2.8 \mathrm{~Hz}, i-C-\mathrm{P}^{2} \mathrm{Ph}_{3}\right), 134.4\left(\mathrm{~d},{ }^{2} J_{\mathrm{CP}}=13.7\right.$ $\left.\mathrm{Hz}, o-C-\mathrm{P}^{1} \mathrm{Ph}_{3}\right), 134.2\left(\mathrm{~d},{ }^{2} J_{\mathrm{CP}}=12.7 \mathrm{~Hz}, o-C-\mathrm{P}^{2} \mathrm{Ph}_{3}\right), 57.8\left(\mathrm{dd},{ }^{2} J_{\mathrm{CP}}=17.7 \mathrm{~Hz},{ }^{2} J_{\mathrm{CP}}=2.0 \mathrm{~Hz}\right.$, C1), $53.2\left(\mathrm{dd},{ }^{2} J_{\mathrm{CP}}=17.7 \mathrm{~Hz},{ }^{2} J_{\mathrm{CP}}=2.9 \mathrm{~Hz}\right), 50.4\left(-\mathrm{COOCH}_{3}\right), 20.1(\mathrm{vt}, J=3.5 \mathrm{~Hz}$, ). 
${ }^{31} \mathrm{P}\left\{{ }^{1} \mathrm{H}\right\} \mathrm{NMR}\left(162 \mathrm{MHz}, \mathrm{C}_{6} \mathrm{D}_{6}, \mathrm{RT}\right): \delta=36.6\left(\mathrm{~d},{ }^{2} J_{\mathrm{PP}}=34.0 \mathrm{~Hz}, P^{2} \mathrm{PPh}_{3}\right), 30.6\left(\mathrm{~d},{ }^{2} J_{\mathrm{PP}}=34.0\right.$ $\left.\mathrm{Hz}, P^{1} \mathrm{PPh}_{3}\right)$.

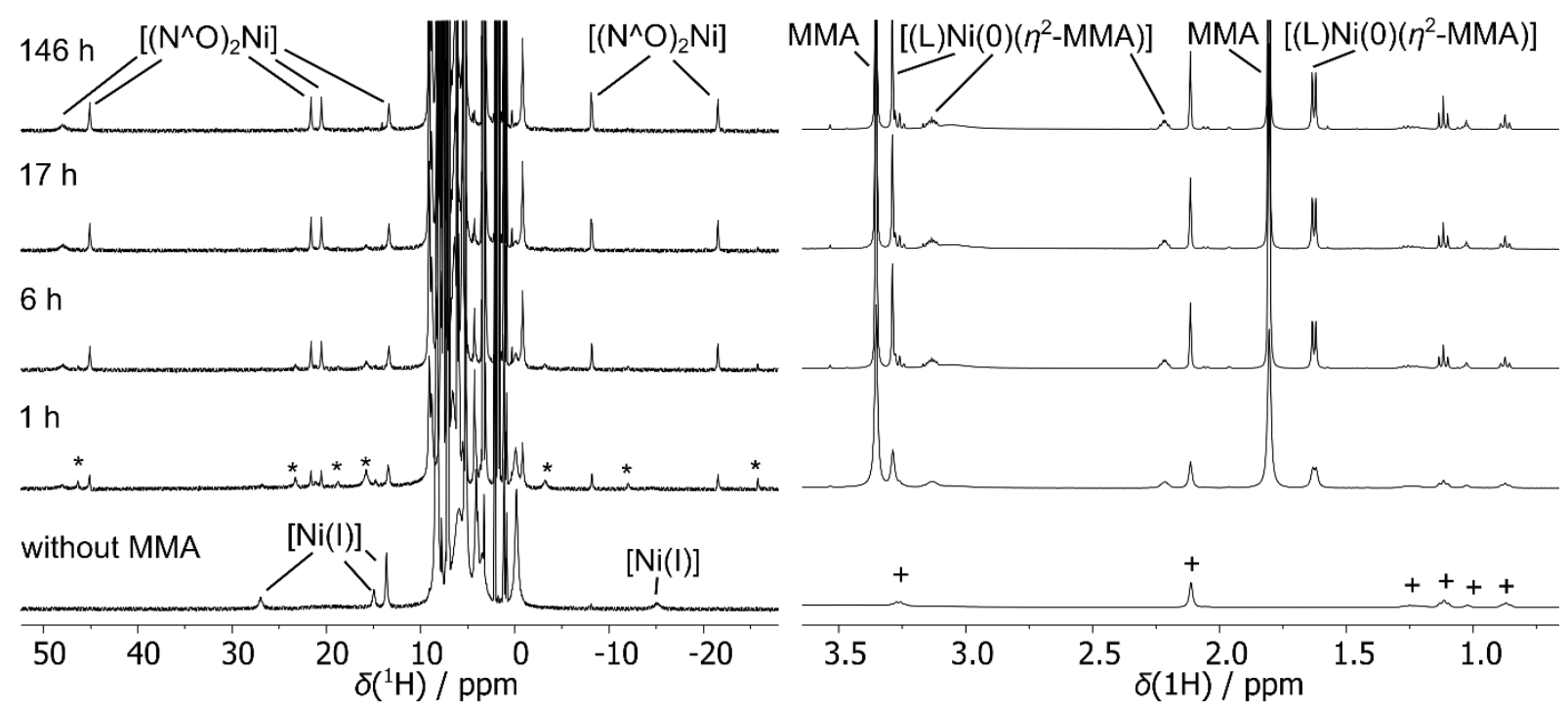

Figure S 51. ${ }^{1} \mathrm{H}$ NMR spectrum $\left(400 \mathrm{MHz}, \mathrm{C}_{6} \mathrm{D}_{6}, \mathrm{RT}\right)$ of the reaction of $3^{\mathrm{PPh} 3}{ }_{\mathrm{PPh} 3}$ with 2 equiv. of MMA at RT after 0 (without MMA, bottom), 1, 6, 17 and $146 \mathrm{~h}$. The left spectrum is showing a spectral window from -30 to $50 \mathrm{ppm}$ and a labeling of paramagnetic compounds. An unassigned intermediate compound is labeled with asterisks (*). The right spectrum is showing the aliphatic region, peaks labeled with a + in the bottom spectrum are solvent impurities. [ $\left.\left(\mathrm{PPh}_{3}\right)_{2} \mathrm{Ni}\left(\eta^{2}-\mathrm{H}_{2} \mathrm{C}=\mathrm{C}\left(\mathrm{CH}_{3}\right) \mathrm{COOMe}\right)\right]$ was the sole detectable reaction product of MMA.

\section{Insertion of MMA into Ni-alkyl catalytic precursors.}

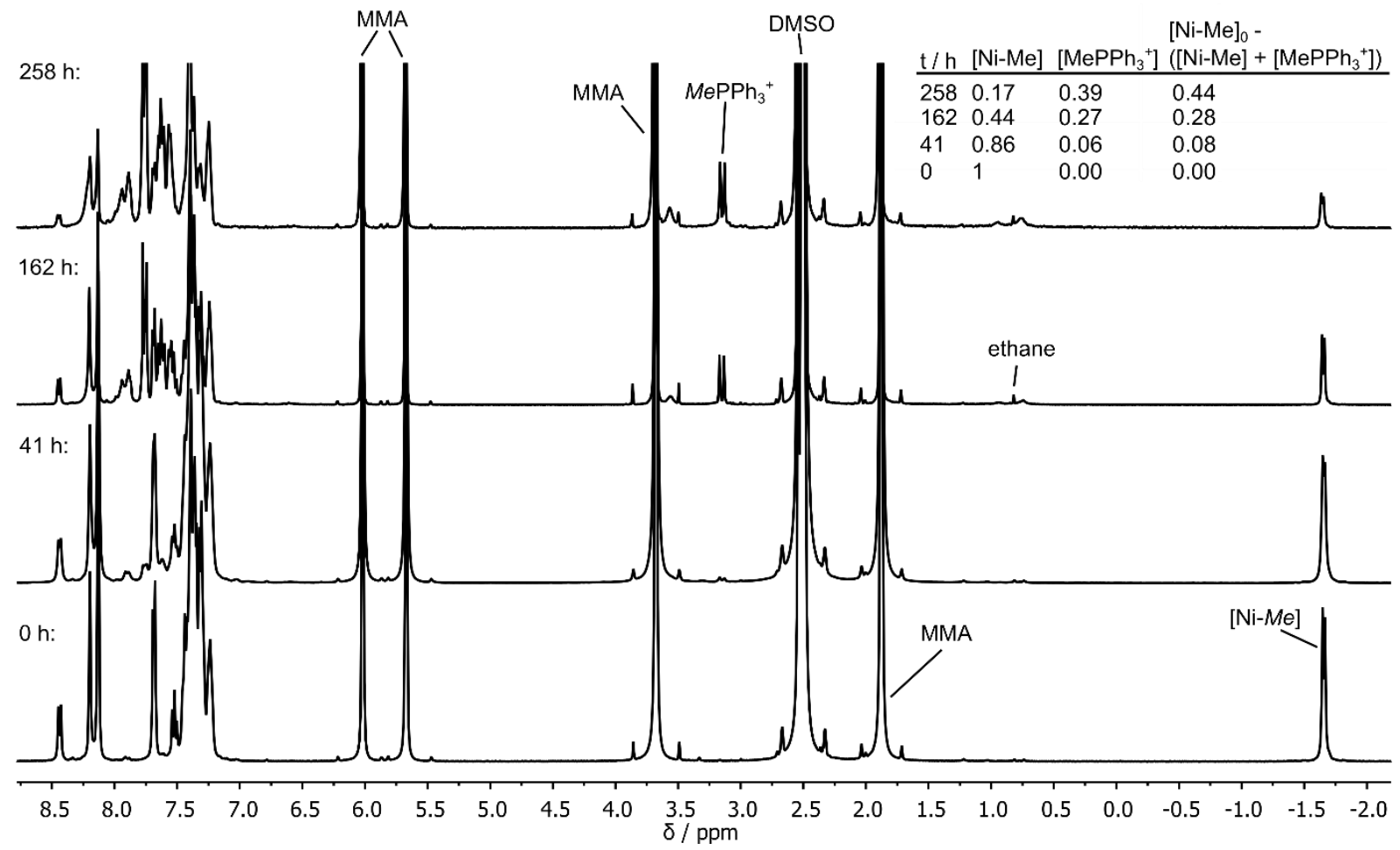

Figure S 52. Monitoring of the reaction of $\mathbf{1}^{\mathrm{Me}}$ dmso $(23 \mathrm{mM}), 2$ equiv. of $\mathrm{PPh}_{3}$ and 7 equiv. of MMA at RT in DMSO- $d_{6}$ by ${ }^{1} \mathrm{H}$ NMR (400 MHz, RT) spectroscopy. The table shows the relative concentrations of $\mathbf{1}^{\mathrm{Me}}{ }_{\mathrm{PPh} 3}$ and $\mathrm{MePPh}_{3}{ }^{+}$normalized to $\left[\mathbf{1}^{\mathrm{Me}}{ }_{\mathrm{PPh} 3}\right]_{0}$ and the difference between $\left[\mathbf{1}^{\mathrm{Me}}{ }_{\mathrm{PPh} 3}\right]_{0}$ and the sum of the normalized concentrations of $\mathbf{1}^{\mathrm{Me}}{ }_{\mathrm{PPh} 3}$ and $\mathrm{MePPh}_{3}{ }^{+}$for each entry. The reductive elimination of the methyl moiety to the phosphine ligand and formation of ethane via bimolecular coupling are the main reaction pathways. In addition, small amounts PMMA were observed. 
To study the reductive elimination of the alkyl moiety to the labile phosphine ligand $\left(\mathrm{PPh}_{3}\right)$ DMSO- $d_{6}$ was used as solvent, because $\mathrm{MePPh}_{3}{ }^{+}$is not soluble in non-polar solvents such as toluene- $d_{8}$ or benzene- $d_{6}$ (see Figure S 53 ). DMSO herein acts as very weak ligand and gets easily and fully displaced by the stronger coordinating aryl phosphine. This is evidenced by the presence of only one [Ni-Me] species with a doublet splitting to the cis coordinating phosphine (-1.6 ppm) in the ${ }^{1} \mathrm{H}$ NMR spectrum of $\mathbf{1}_{\text {dmso }}^{\mathrm{Me}}$ in presence of 2 equiv. $\mathrm{PPh}_{3}$ (Figure S 52).

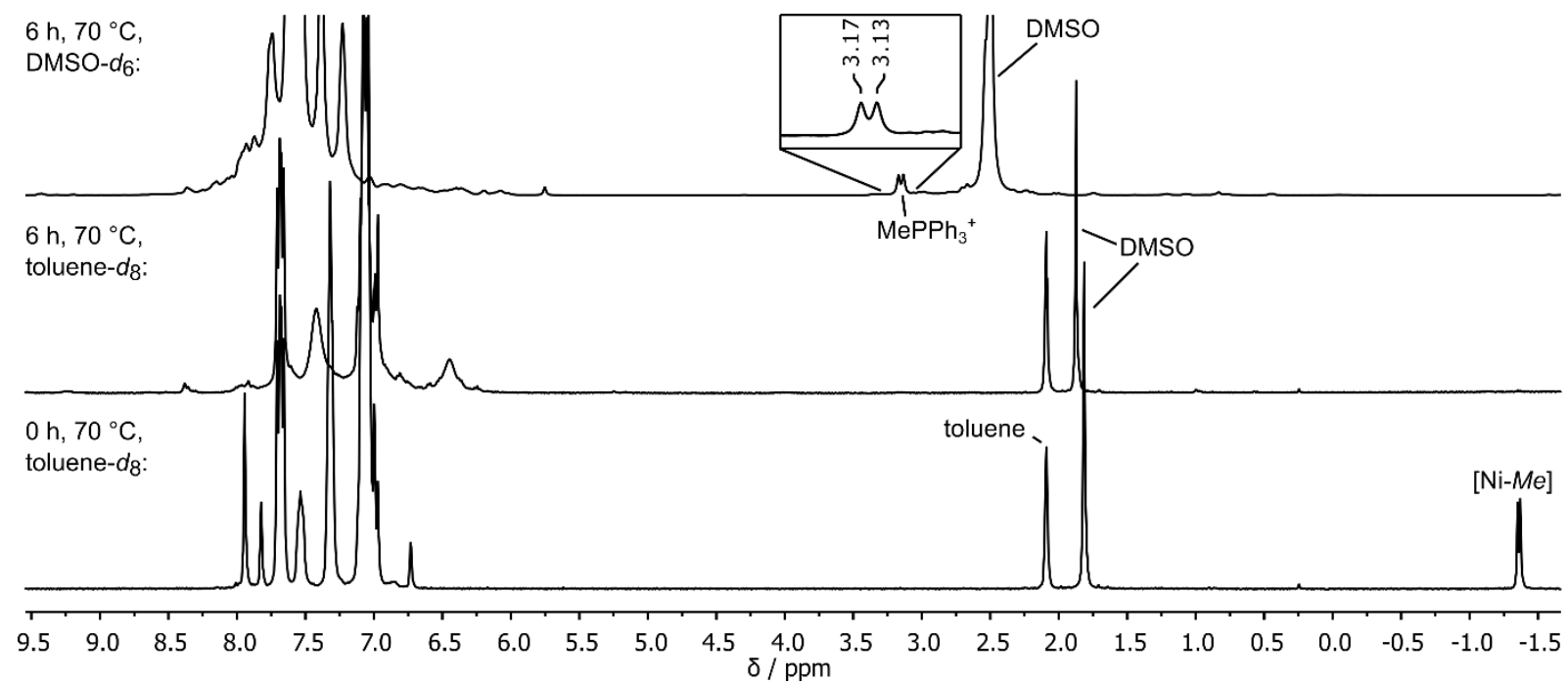

Figure S 53. Monitoring the reaction of $\mathbf{1}^{\mathrm{Me}}{ }_{\text {dmso }}(18 \mathrm{mM})$ and 4 equiv. of $\mathrm{PPh}_{3}$ in toluene- $d_{8}$ at $70{ }^{\circ} \mathrm{C}$ by ${ }^{1} \mathrm{H} \mathrm{NMR}$ spectroscopy $(400 \mathrm{MHz})$ after $0 \mathrm{~h}$ and $6 \mathrm{~h}$ (bottom and middle). No formation of reaction products in the aliphatic region was observed. By evaporation of toluene- $d_{8}$ and dissolving the residue in DMSO- $d_{6}(400 \mathrm{MHz}, \mathrm{RT})$, $\mathrm{MePPh}_{3}{ }^{+}$was identified $\left(3.15 \mathrm{ppm}, \mathrm{d},{ }^{2} J_{\mathrm{HP}}=14.7 \mathrm{~Hz}\right.$, upper spectrum) as major decomposition product (approx. $40 \%$ with respect to [Ni-Me], value was derived from ${ }^{31} \mathrm{P}\left\{{ }^{1} \mathrm{H}\right\}$ spectroscopy vs. $\mathrm{OPPh}_{3}$ as internal standard).

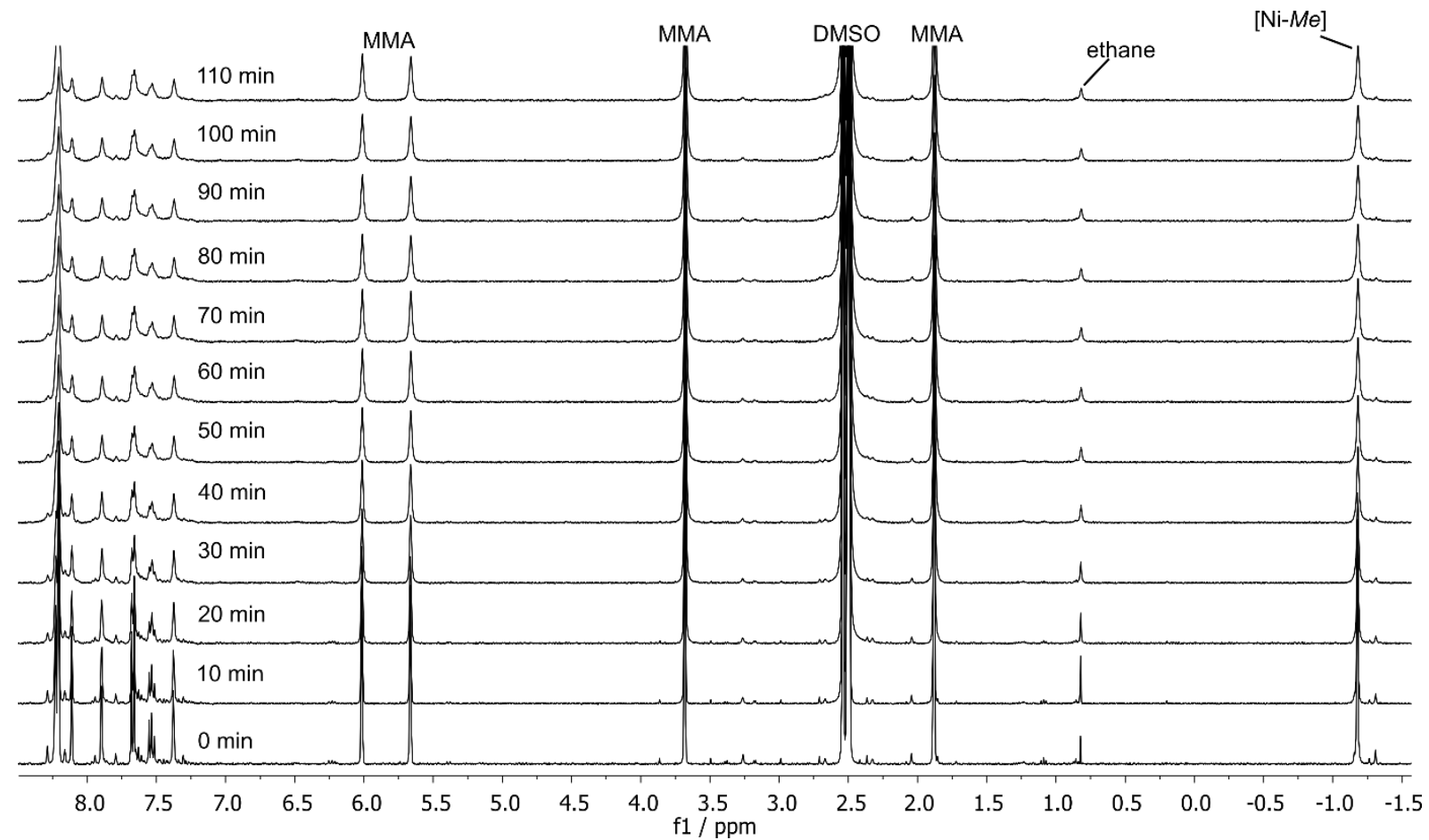

Figure S 54. Time dependent ${ }^{1} \mathrm{H}$ NMR ( $400 \mathrm{MHz}$, DMSO- $\left.d_{6}, 50{ }^{\circ} \mathrm{C}\right)$ spectra showing the reaction of $\mathbf{1}^{\mathrm{Me}} \mathrm{dmso}_{\mathrm{dmo}}$ with 2 equiv. of MMA. The formation of ethane was the only detectable organic reaction product. Higher temperatures led to MMA polymerization and severely broadened resonances. 
a)

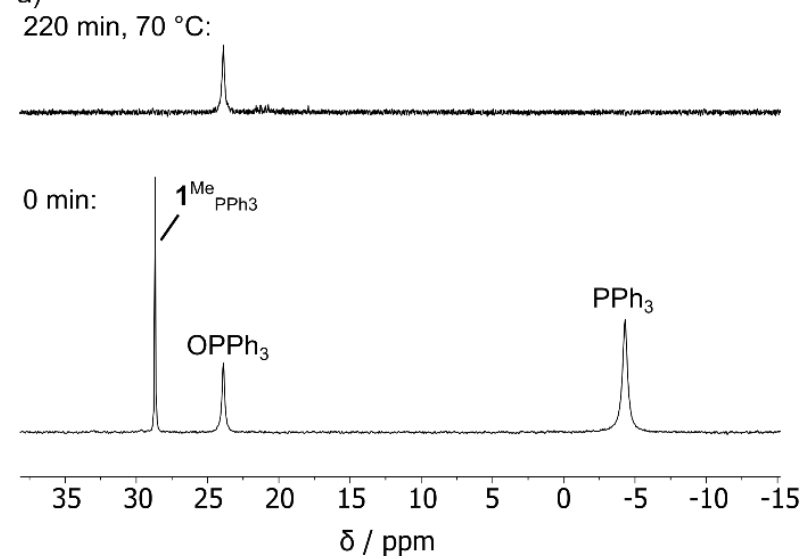

b)

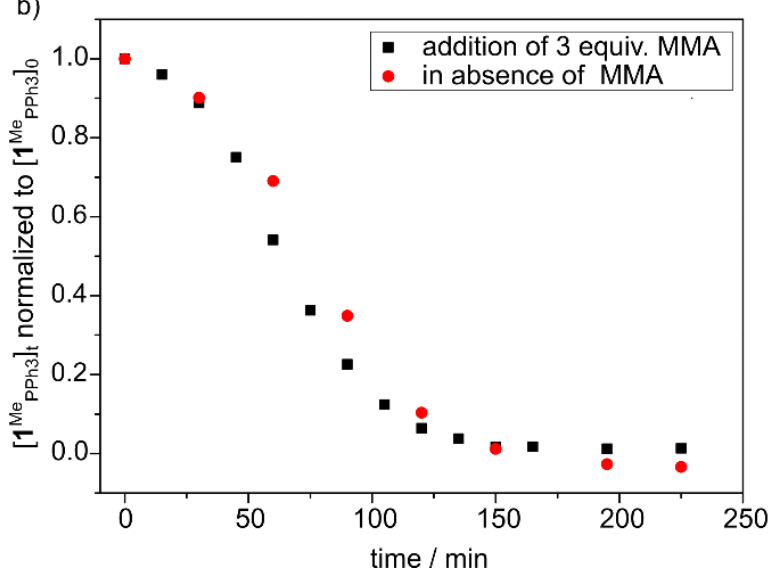

Figure S 55. Monitoring the reaction of $\mathbf{1}^{\mathrm{Me}}{ }_{\mathrm{PPh} 3}(18 \mathrm{mM})$ and 3 equiv. of $\mathrm{PPh}_{3}$ with and without additional 3 equiv. of MMA at $70{ }^{\circ} \mathrm{C}$ in toluene- $d_{8}$. (a) ${ }^{31} \mathrm{P}$ NMR spectra $(162 \mathrm{MHz}, \mathrm{RT})$ after $0 \mathrm{~min}$ (lower) and after 220 minutes at $70{ }^{\circ} \mathrm{C}$ (upper). (b) The decline of $\mathbf{1}^{\mathrm{Me}} \mathrm{PPh}_{3}$ resonance with time for the reaction with 3 equiv. of MMA (black) and without additional MMA (red). The observed decays of [Ni-Me] are almost independent on the presence of MMA.

Low temperature insertion experiments of MMA into $\mathbf{1}^{H_{P M e 3}}$.

Insertion experiments were carried out with in-situ prepared $\mathbf{1}^{\mathrm{H}_{\mathrm{PMe}}}$ as well as with isolated $\mathbf{1}^{\mathrm{H}}{ }_{\mathrm{PMe}}$. In previous experiments, it was found that the presence of the hydride source $\left(\mathrm{NaHBOMe}_{3}\right.$ ) does not influence the reactivity of $\mathbf{1}^{\mathrm{H}_{\mathrm{PMe}}}$ towards MA. ${ }^{10,35}$

Procedure for the observation of the decomposition of $\mathbf{1}^{M M A H_{P P h 3}}$

In a Schlenk-flask $1^{\mathrm{Cl}_{\mathrm{PMe}}}$ (0.06 mmol, $63 \mathrm{mg}, 1$ equiv.) and $\mathrm{NaHB}(\mathrm{OMe})_{3}(0.18 \mathrm{mmol}, 23 \mathrm{mg}$, 3 equiv.) were cooled to $-30{ }^{\circ} \mathrm{C}$ in an isopropanol/dry ice bath and slowly dissolved in THF $(5 \mathrm{~mL})$. The reaction was stirred for $30 \mathrm{~min}$, MMA ( $0.3 \mathrm{~mL}, 2.8 \mathrm{mmol}$, 47 equiv.) was slowly added to the brown suspension and the mixture was stored for $12 \mathrm{~h}$ at $-25^{\circ} \mathrm{C}$. Subsequently, the solvent and excess MMA was removed under reduced pressure while cooling to $0{ }^{\circ} \mathrm{C}$. The resulting red solid was washed with cold pentane at $-78^{\circ} \mathrm{C}$ and dried in vacuo. In a $\mathrm{N}_{2}$ filled glovebox $18 \mathrm{mg}$ of the red solid was transferred into a NMR tube, sealed with a rubber septum, removed from the glovebox, cooled to $-78^{\circ} \mathrm{C}$ and THF- $d_{8}(0.5 \mathrm{~mL})$ was slowly added through a rubber septum using a gas tight syringe. The tube was shaken and transferred to the precooled NMR probe at the desired temperature.

Characteristic NMR resonances for $\mathbf{1}^{\mathrm{MMAH}}{ }_{\mathrm{PPh} 3}$ :

${ }^{1} \mathrm{H}$ NMR $\left(400 \mathrm{MHz}, \mathrm{THF}-d_{8},-35{ }^{\circ} \mathrm{C}\right): \delta=8.73\left(\mathrm{~d},{ }^{4} J_{H P}=8.2 \mathrm{~Hz}, 1 \mathrm{H}, \mathrm{H} 7\right), 8.31(\mathrm{~s}, 2 \mathrm{H}, \mathrm{H} 20)$, 8.28 (s, 1H, H17), 8.19 (s, 1H, H22), 8.11 (s, 2H, H15), $7.96\left(\mathrm{~d},{ }^{4} J_{\mathrm{HH}}=2.2 \mathrm{~Hz}, 1 \mathrm{H}, 4\right), 7.46(\mathrm{~d}$, $\left.{ }^{4} J_{H H}=2.2 \mathrm{~Hz}, 1 \mathrm{H}, \mathrm{H} 6\right), 3.44(\mathrm{~s}, 3 \mathrm{H}, \mathrm{H} \varepsilon), 1.80(\mathrm{~m}, 1 \mathrm{H}, \mathrm{H} \beta), 1.24(\mathrm{~m}, 3 \mathrm{H}, \mathrm{H} \gamma), 1.00\left(\mathrm{~d},{ }^{2} J_{H P}=\right.$ $\left.10.3 \mathrm{~Hz}, 9 \mathrm{H}, \mathrm{P}-\mathrm{CH} \mathrm{H}_{3}\right),-0.40\left(\mathrm{~m}, 1 \mathrm{H}, \mathrm{H \alpha} \alpha^{\prime}\right),-0.81\left(\mathrm{t},{ }^{2} \mathrm{JHH}_{\mathrm{HH}}=9.9 \mathrm{~Hz}, 1 \mathrm{H}, \mathrm{H \alpha} "\right)$ ppm. 
${ }^{13} \mathrm{C}\left\{{ }^{1} \mathrm{H}\right\}$ NMR (100 MHz, THF- $\left.d_{8},-35^{\circ} \mathrm{C}\right): \delta=176.9$ (-COOMe), 167.0 (C7), 163.7 (C2), 50.2 (C4), 143.1 (C6), 132.2 (C20), 131.7 (C15), 122.4 (C17, C22), 120.7 (C1), 51.2 (C $)$ ), 41.7 (Cß), $21.1(\mathrm{C} \gamma), 12.5\left(\mathrm{P}-\mathrm{CH}_{3}\right), 8.8(\mathrm{C} \alpha) \mathrm{ppm}$.

${ }^{31} \mathrm{P}\left\{{ }^{1} \mathrm{H}\right\}$ NMR $\left(162 \mathrm{MHz}, \mathrm{THF}-d_{8},-60^{\circ} \mathrm{C}\right): \delta=-5.7$ (s) ppm.

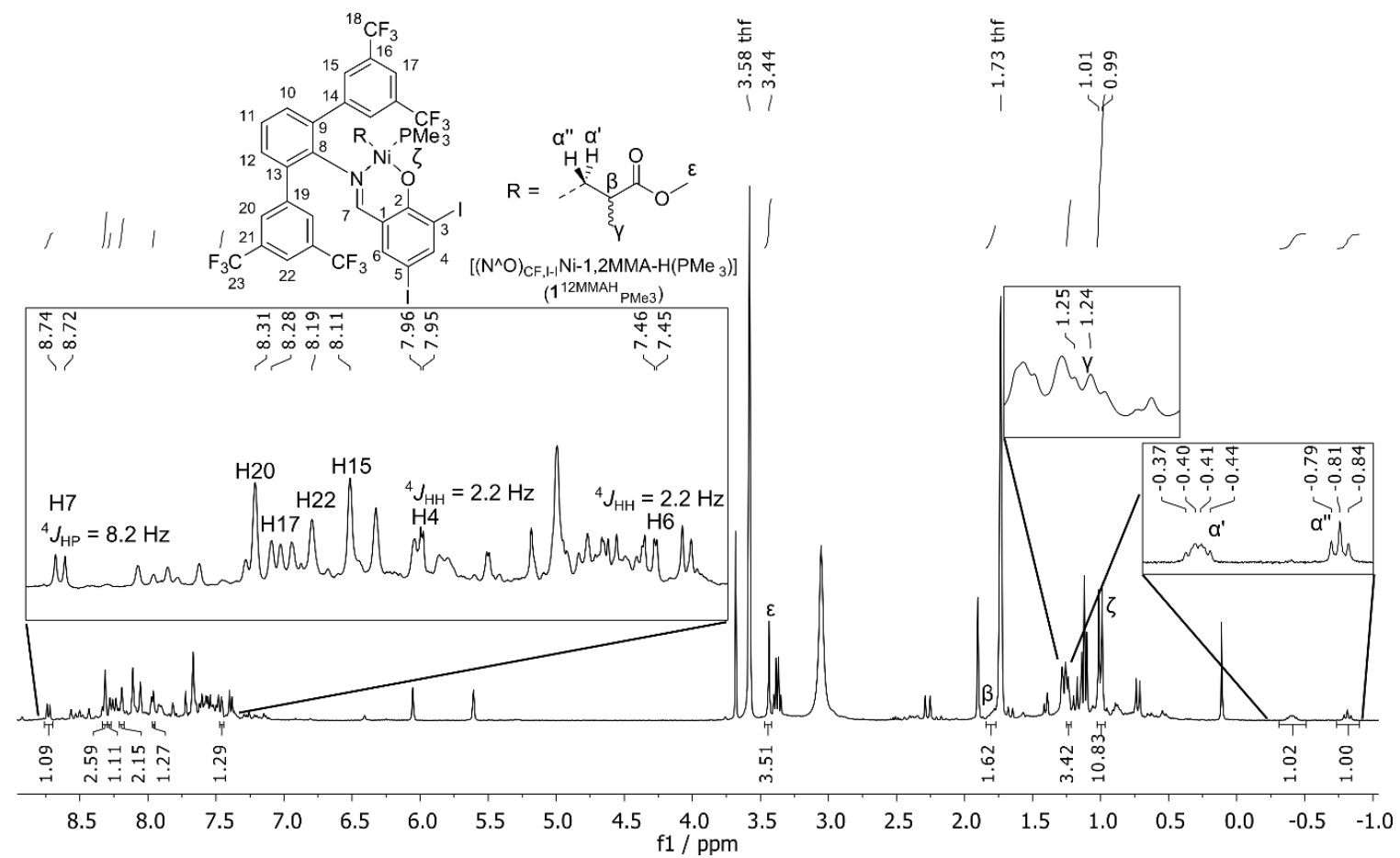

Figure S 56. ${ }^{1} \mathrm{H}$ NMR spectra $\left(400 \mathrm{MHz}, \mathrm{THF}-d_{8},-35^{\circ} \mathrm{C}\right)$ showing the reaction of $\mathbf{1}^{\mathrm{H}_{\mathrm{PMe}}}$ with 2 equiv. of MMA after $12 \mathrm{~h}$ at $-35^{\circ} \mathrm{C}$.

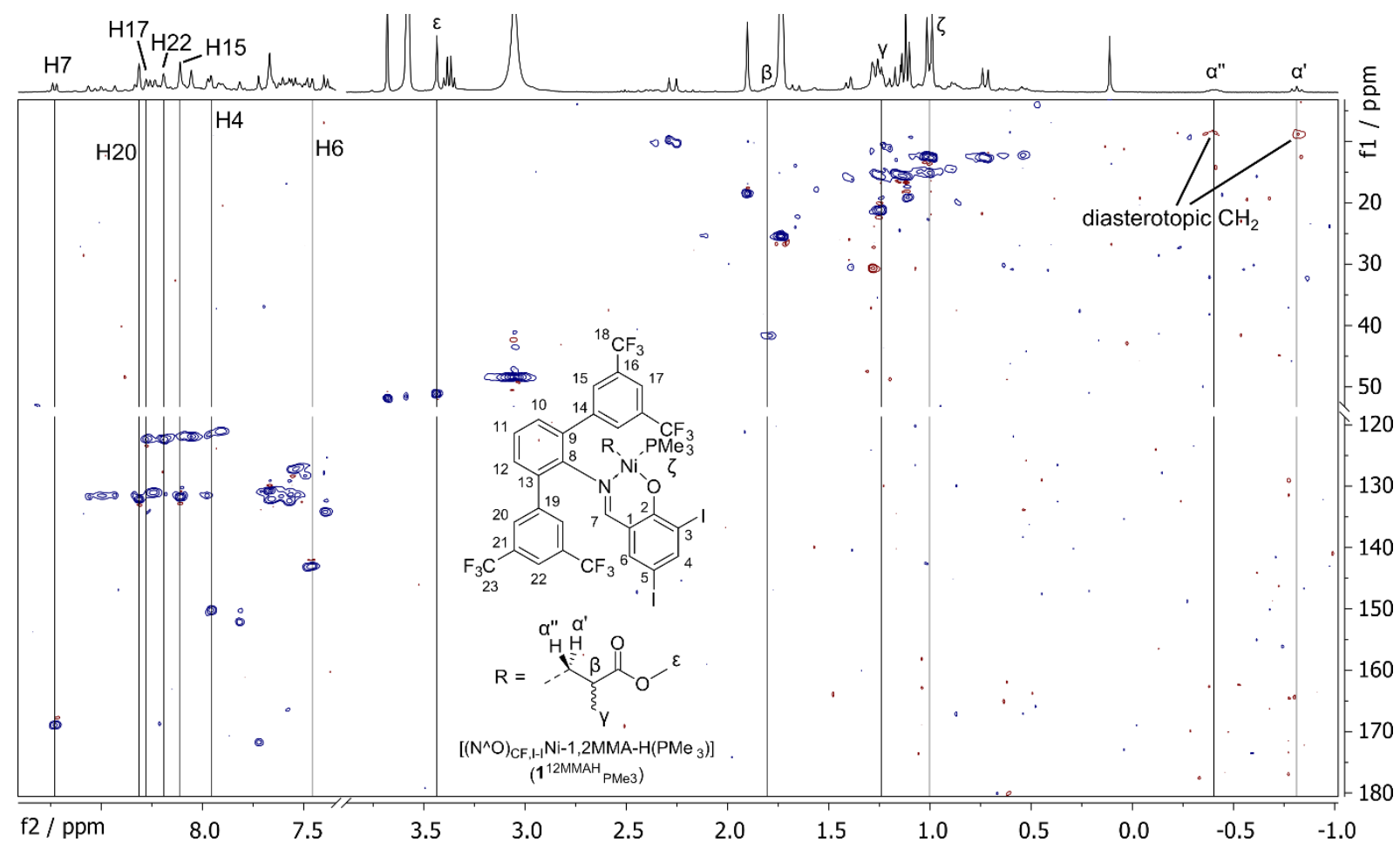

Figure S 57. ${ }^{1} \mathrm{H}-{ }^{13} \mathrm{C}$ gHSQCAD $\left(400 \mathrm{MHz}, \mathrm{THF}-d_{8},-35^{\circ} \mathrm{C}\right)$ showing the reaction of $\mathbf{1}^{\mathrm{H}}{ }_{\mathrm{PMe}}$ with 2 equiv. of MMA after $12 \mathrm{~h}$ at $-35^{\circ} \mathrm{C}$. 


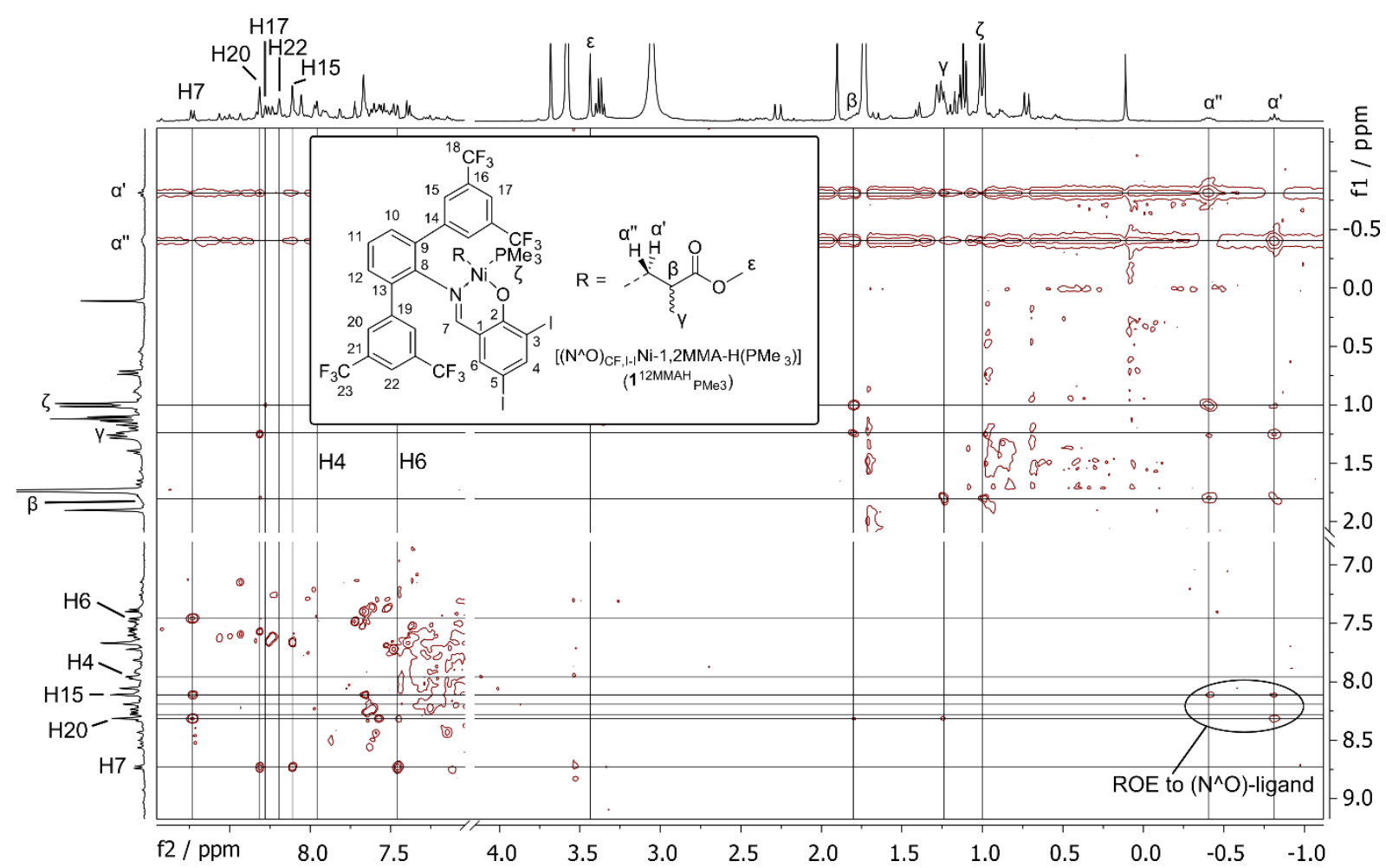

Figure S 58. ${ }^{1} \mathrm{H}-{ }^{1} \mathrm{H}$ ROESYAD $\left(400 \mathrm{MHz}\right.$, THF- $d_{8},-35{ }^{\circ} \mathrm{C}, 250 \mathrm{~ms}$ spin lock) showing the reaction of $\mathbf{1}^{\mathrm{H}} \mathrm{PMe}_{3}$ with 2 equiv. of MMA after $12 \mathrm{~h}$ at $-35^{\circ} \mathrm{C}$. A ROE of $\alpha, \beta$ and $\gamma$ protons from the inserted MMA moiety to $\left(\mathrm{N}^{\wedge} \mathrm{O}\right)$ protons $\mathrm{H} 20$ and H15 was observed. Negative contours are not shown for clarity.

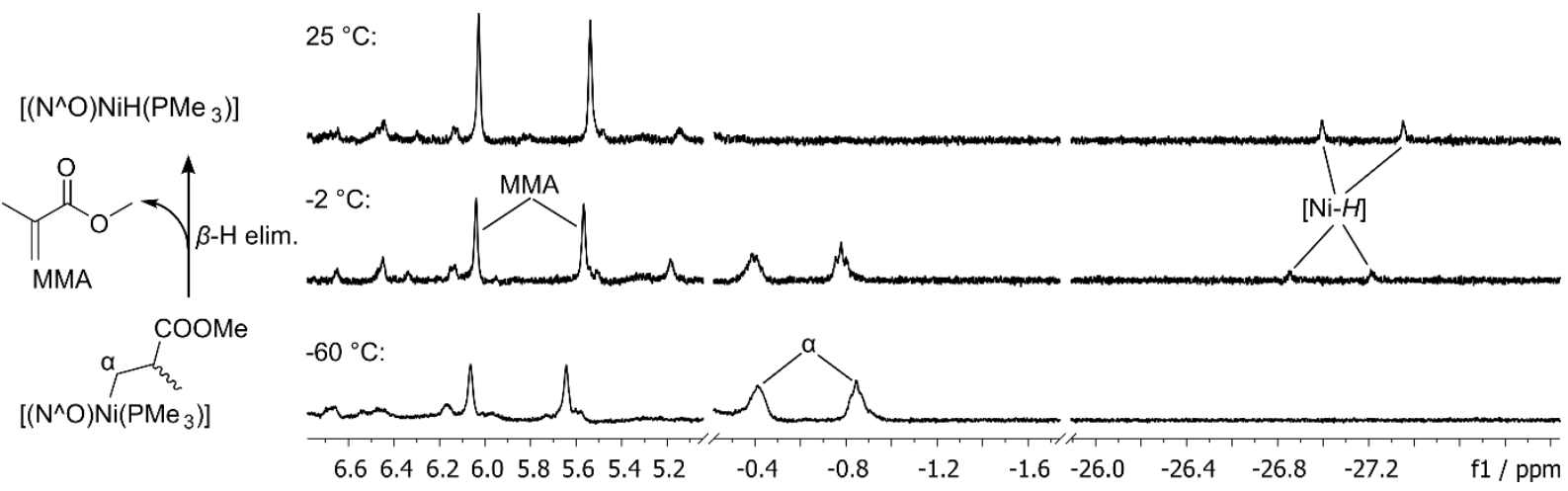

Figure S 59. ${ }^{1} \mathrm{H}$ NMR spectra $\left(400 \mathrm{MHz}, \mathrm{THF}-d_{8}\right)$ showing the $\beta$-H elimination at variable temperatures $(-60,-2$ and $25^{\circ} \mathrm{C}$ ). Starting from $\mathbf{1}^{\mathrm{MMAH}}{ }_{\mathrm{PMe} 3}$ forming $\mathbf{1}_{\mathrm{PMe} 3}$ and MMA. The reversibility of the insertion of MMA was observed by the formation of $[\mathrm{Ni}-\mathrm{H}]$ (d, approx. $-27 \mathrm{ppm})$. 


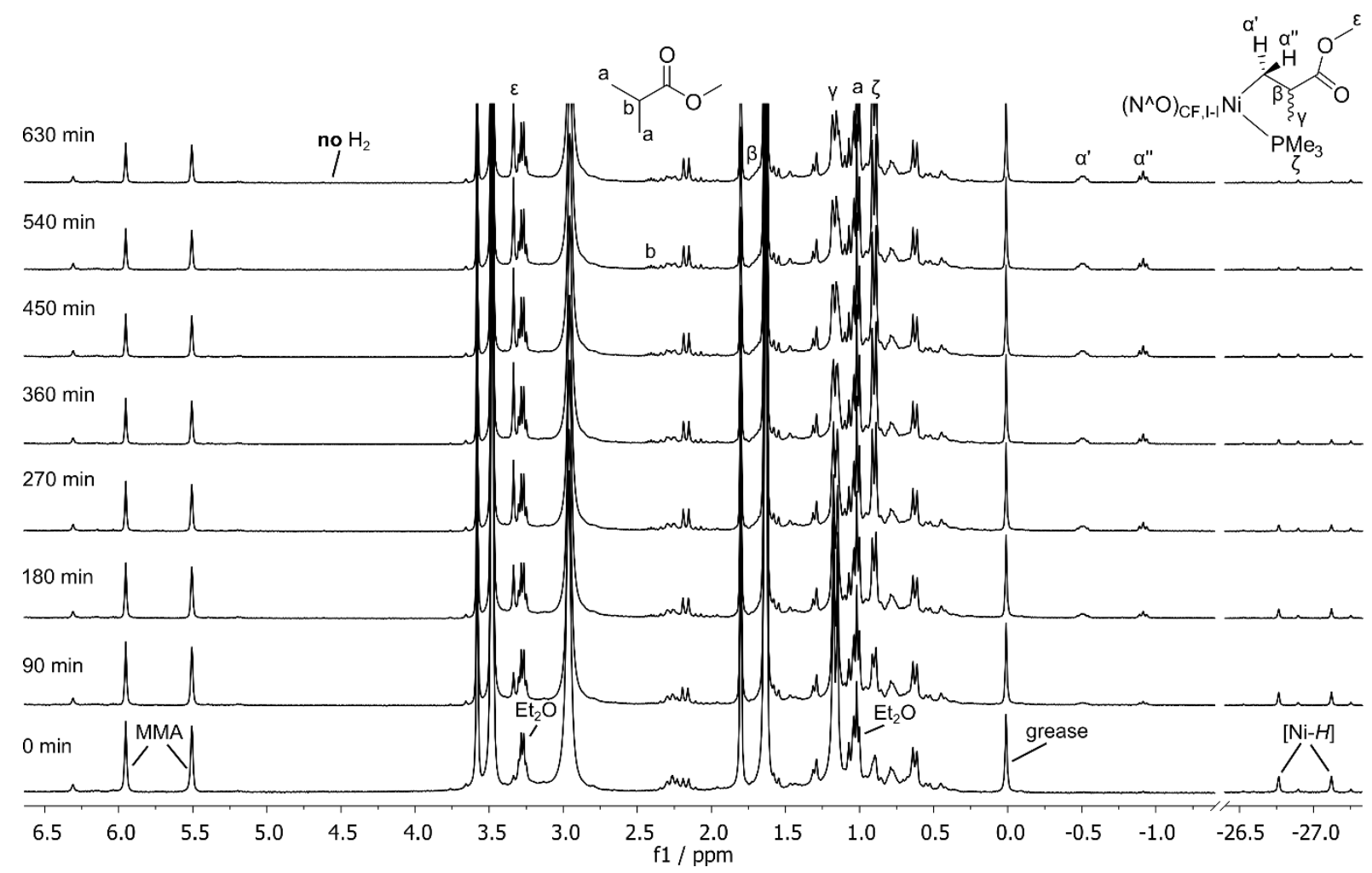

Figure S 60. Time dependent ${ }^{1} \mathrm{H}$ NMR $\left(400 \mathrm{MHz}, \mathrm{THF}-d_{8},-35^{\circ} \mathrm{C}\right)$ spectra showing the reaction of $\mathbf{1}^{\mathrm{H}}{ }_{\mathrm{PMe} 3}(16$ $\mu$ mol) with 2 equiv. of MMA. 


\section{Sources of Organic Free Radicals in $\left(\mathrm{N}^{\wedge} \mathrm{O}\right) \mathrm{Ni}(\mathrm{II}) \mathrm{R}(\mathrm{L})$ Mediated Polymerizations}

\subsection{Aryl phosphine as non-innocent ligand.}

Discussion of additional pathways for the transfer or an aryl group from PAr3 to the PMMA chain end.

(a) One possibility is an addition of an initially generated organic radical to $\mathrm{PAr}_{3},{ }^{38}$ forming an unstable phosphoranyl radical. A subsequent $\mathrm{P}-\mathrm{C}_{\mathrm{Ar}}$ bond cleavage would lead to a radical scrambling. However, the initial free radical would be expected to also react with MMA (used as a solvent) and thus form specific end-groups in the PMMA obtained. This would be indicative of this initial free radical. However, NMR spectroscopy as well as MALDI-TOF MS of the PMMA obtained did not lead to the identification of end-groups which could be attributed to an initially organic radical species other than phenyl groups. Therefore, we exclude this pathway.

(b) In stoichiometric experiments the reductive elimination of a methyl group from [Ni-Me] to $\mathrm{PPh}_{3}$ was found to occur to a significant extent (vide supra). A reversible reaction ('oxidative addition') could be possible ${ }^{39}$ and could result in a transfer of a $\mathrm{Ph}$ moiety to the Ni-center, and potentially ultimately to the PMMA chains. Phosphonium species (e.g. $\mathrm{MePPh}_{3}{ }^{+}$) and lowvalent $\mathrm{Ni}(0)$ species would herein represent the active species. However, MMA homopolymerizations with $\mathrm{MePPh}_{3}{ }^{+}$added to different $\left[\left(\mathrm{N}^{\wedge} \mathrm{O}\right) \mathrm{Ni}(\mathrm{II}) \mathrm{R}\left(\mathrm{PPh}_{3}\right)\right]$ complexes and MMA did not show an increased efficiency for radical polymerizations $\left(70{ }^{\circ} \mathrm{C}, 0.5 \mathrm{mmol} \cdot \mathrm{L}^{-1}\right.$ of $\mathbf{1}^{\mathrm{Ph}}{ }_{\mathrm{PPh} 3}$, neat MMA, $162 \mathrm{~min}$, with or without 1.2 equiv. $\mathrm{MePPh} 3 \mathrm{Br}, 2.8 \%$ yield, respectively). Also, exposure of a mixture of $\left[\mathrm{Ni}(0)\left(\mathrm{PPh}_{3}\right)_{4}\right]$ plus 2 equiv. $\mathrm{MePPh}_{3} \mathrm{I}$ to $\mathrm{MMA}$ only resulted in trace amounts of PMMA $\left(70{ }^{\circ} \mathrm{C},[\mathrm{Ni}]=0.5 \mathrm{mmol} \cdot \mathrm{L}^{-1}\right.$, neat MMA, $225 \mathrm{~min}$, Figure S 65 blue). In addition, a scrambling of P substituents at the Ni-center was not observed by NMR spectroscopy. These results suggest that this pathway can also be excluded.

General Procedure for the generation of alkyl and aryl spin adducts of N-tert-butyl- $\alpha$ phenylnitrone (PBN).

A modified procedure published by Kotake et al. in 1988 for the preparation of Ph-PBN was used for the preparation of Ph-PBN and Me-PBN spin adducts. ${ }^{40}$ Here, PBN was reacted with the corresponding alkyl- or aryl-lithium compound. 
To a solution of PBN (2.3 mg, $10 \mu \mathrm{mol}, 1$ equiv.) in benzene ( $1 \mathrm{~mL}), 100$ equiv. of $\mathrm{PhLi} / \mathrm{MeLi}$ were slowly added and the solution was stirred for $15 \mathrm{~min}$ at RT. Water ( $2 \mathrm{~mL}$ ) was slowly added, the aqueous phase was separated and pressurized air was bubbled through the solution for $1 \mathrm{~h}$. The organic phase was washed with water $(2 \mathrm{~mL})$ and reduced to dryness under vacuum. The resulting solid was dissolved in degassed toluene $(4 \mathrm{~mL})$, transferred into a $3 \mathrm{~mm}$ EPR tube, the tube was sealed with a rubber septum, fixed with PTFE tape, removed from the glovebox and quickly transferred into the EPR probe.

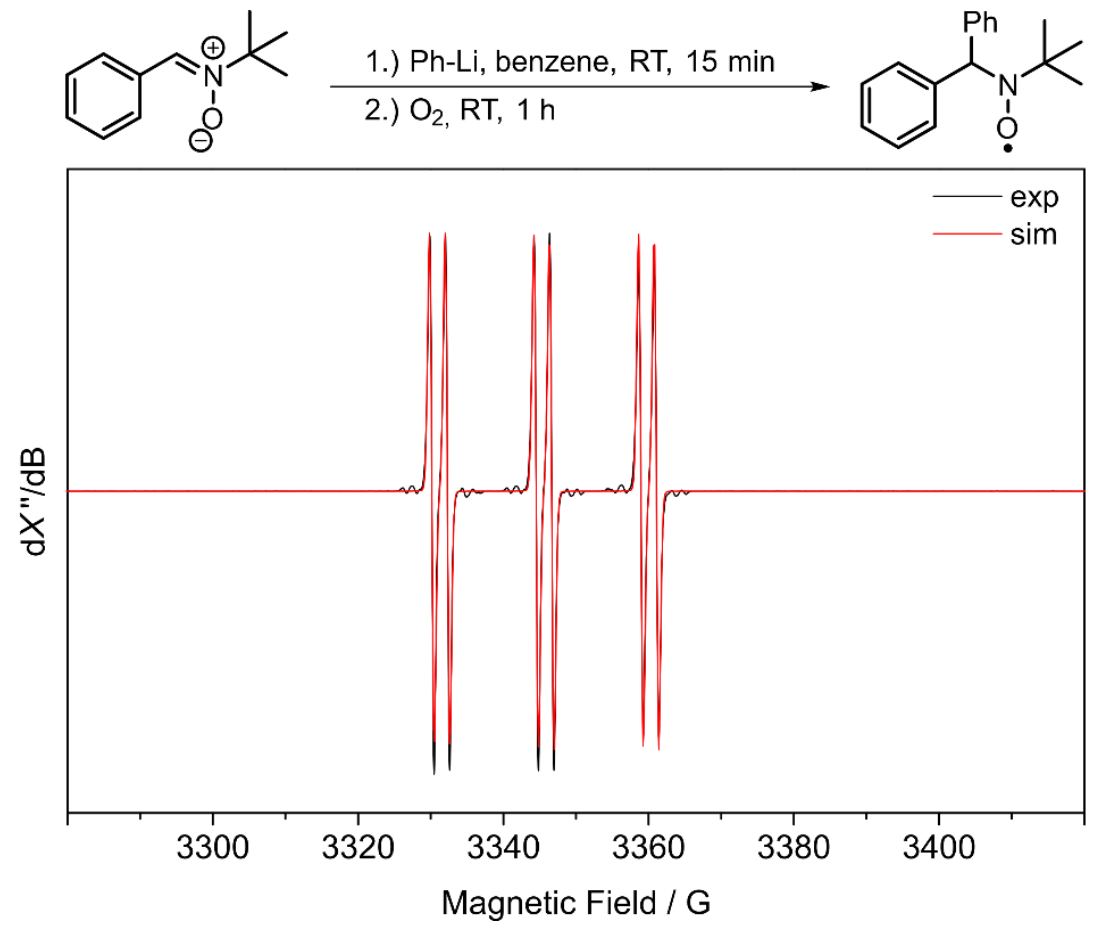

Figure S 61. CW X-band (9.394 GHz) EPR spectrum showing the resonance of the phenyl spin adduct of PBN in toluene at $5{ }^{\circ} \mathrm{C}$. Acquisition parameters: $150 \mathrm{G}$ sweep, $150 \mathrm{~s}$ sweep time, $250 \mathrm{mG}$ modulation, $5 \mathrm{scans}, 0.5 \mathrm{~mW}$ microwave power. The hyperfine splitting of $a_{\mathrm{N}}=14.4 \mathrm{G}$ and $a_{\mathrm{H}}^{\beta}=2.2 \mathrm{G}$ was derived from simulation. 


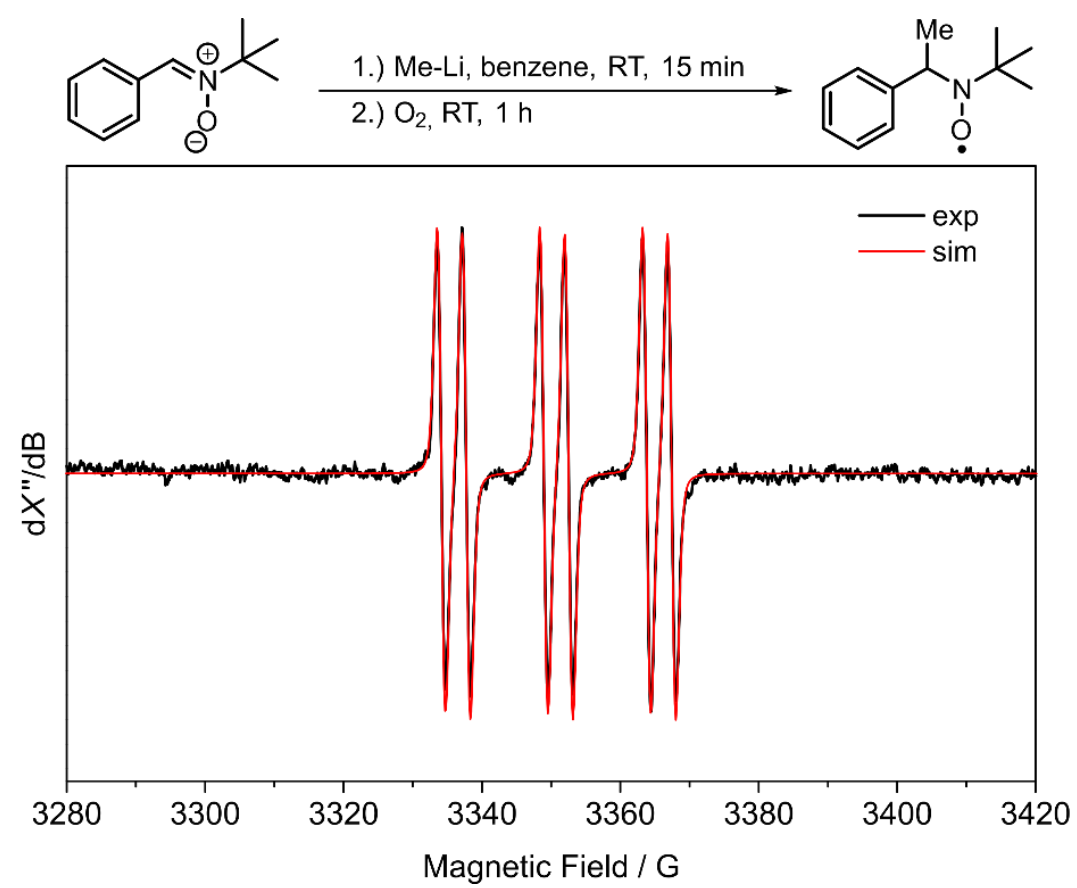

Figure S 62. CW X-Band (9.370 GHz) EPR spectrum showing the resonance of the methyl spin adduct of PBN in toluene at $25{ }^{\circ} \mathrm{C}$. Acquisition parameters: $150 \mathrm{G}$ sweep, $60 \mathrm{~s}$ sweep time, $400 \mathrm{mG}$ modulation, 10 Scans, 2.5 $\mathrm{mW}$ microwave power. The hyperfine splitting of $a_{\mathrm{N}}=14.81 \mathrm{G}$ and $a_{\mathrm{H}}{ }^{\beta}=3.59 \mathrm{G}$ was derived from simulation and agrees with reported values $\left(a_{\mathrm{N}}=14.91 \mathrm{G}\right.$ and $a_{\mathrm{H}}{ }^{\beta}=3.66 \mathrm{G}$ in toluene at RT) ${ }^{41}$

a) ${ }^{1} H$ NMR: $\quad$ b) ${ }^{31} P\left\{{ }^{1} H\right\} N M R$

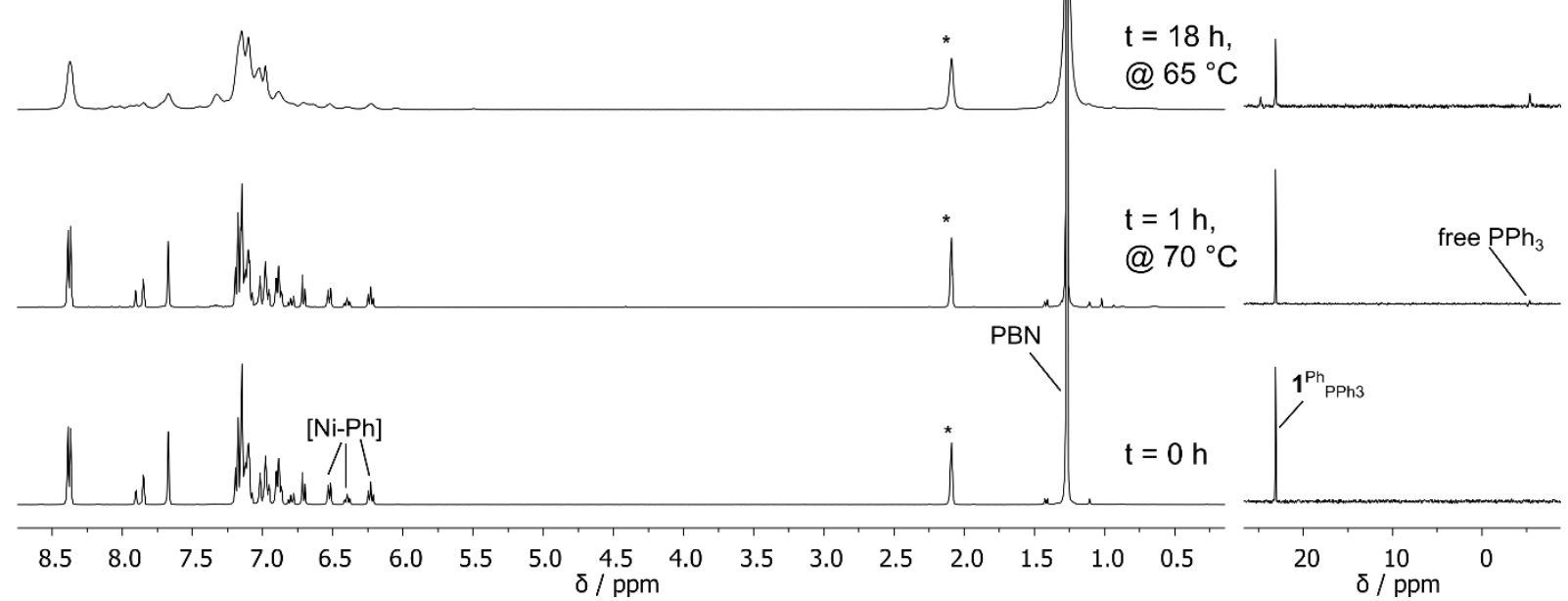

Figure S 63. Monitoring the reaction of $\mathbf{1}^{\mathrm{Ph}}{ }_{\mathrm{PPh} 3}(12 \mathrm{mM})$ with 5 equiv. of PBN in toluene- $d_{8}(*)$ after $0 \mathrm{~h}$ (bottom), $1 \mathrm{~h}$ at $70{ }^{\circ} \mathrm{C}$ (middle) and after $18 \mathrm{~h}$ at $65{ }^{\circ} \mathrm{C}$ (top) by ${ }^{1} \mathrm{H}(400 \mathrm{MHz}, \mathrm{RT})$ and ${ }^{31} \mathrm{P}\left\{{ }^{1} \mathrm{H}\right\}(162 \mathrm{MHz}, \mathrm{RT})$. It is shown that the decomposition of the initial complex $\mathbf{1}^{\mathrm{Ph}} \mathrm{PPh}_{3}$ is not accelerated in the presence of PBN and therefore a reaction of the spin-trap with $\left[\left(\mathrm{N}^{\wedge} \mathrm{O}\right) \mathrm{Ni}(\mathrm{II}) \mathrm{R}\left(\mathrm{PPh}_{3}\right)\right]$ complexes seems unlikely. However, a significant broadening of the spectra was observed after a prolonged time $\left(18 \mathrm{~h}, 65^{\circ} \mathrm{C}\right.$, top $)$, suggesting the presence of free radicals.

To ensure that $\mathrm{PBN}$ does not react with the supplied $\left[\left(\mathrm{N}^{\wedge} \mathrm{O}\right) \mathrm{Ni}(\mathrm{II}) \mathrm{R}\left(\mathrm{PPh}_{3}\right)\right]$ precursor complexes, $\mathbf{1}^{\mathrm{Ph}}{ }_{\mathrm{PPh} 3}$ was heated with 5 equiv. of PBN in toluene- $d_{8}$ up to $70{ }^{\circ} \mathrm{C}$. No acceleration of the decomposition compared to the absence of additional PBN was observed (Figure S 63). 


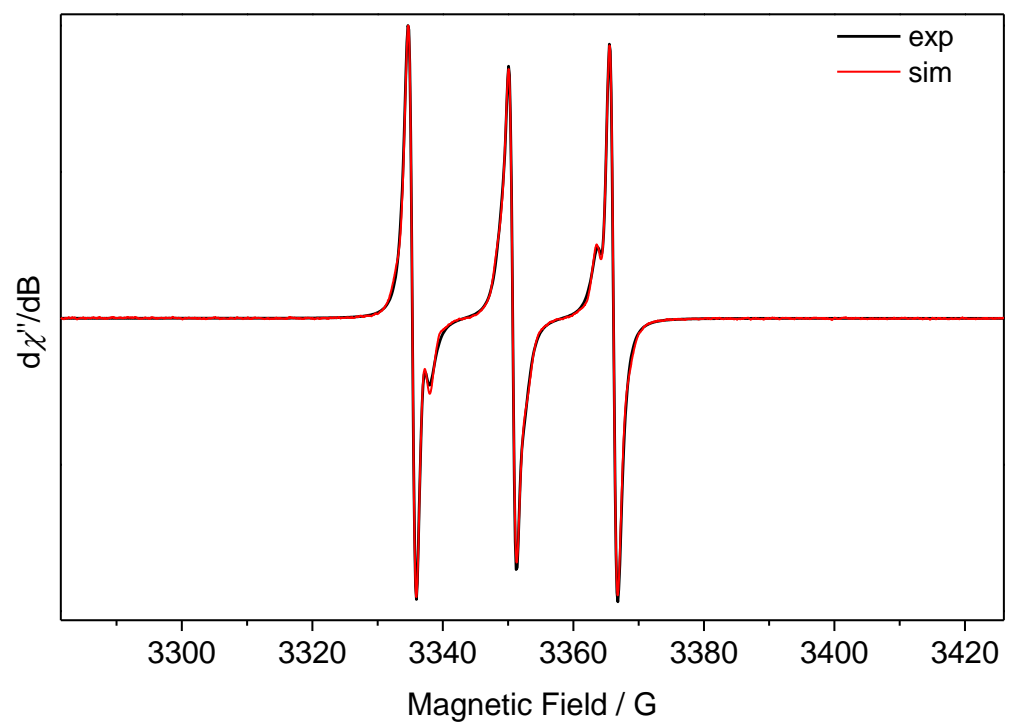

Figure S 64. CW X band (9.371 MHz, RT) EPR spectrum of the PBN spin-trapped radical of the reaction of $\mathbf{1}_{\mathrm{dmso}}^{\mathrm{Me}}(4 \mathrm{mM})$ and 12 equiv. of PBN at $70{ }^{\circ} \mathrm{C}$ after $24 \mathrm{~h}$ in toluene (black). The values of the hyperfine splitting were derived from simulation: First species $-a_{\mathrm{N}, 1}=15.35 \mathrm{G}$, Second species $-a_{\mathrm{N}, 2}=14.65 \mathrm{G}, a_{\mathrm{H}}{ }^{\beta}=2.56 \mathrm{G}$.

\subsection{Reactivity of $\mathrm{Ni}(\mathrm{O})$ and $\mathrm{Ni}(\mathrm{I})$ species.}

A high concentration of the $\left[\left(\mathrm{N}^{\wedge} \mathrm{O}\right) \mathrm{Ni}(\mathrm{I})\right]$ complex was chosen to minimize side reactions with impurities. A short reaction time (in comparison to experiments with $\mathrm{Ni}$ (II) complexes) was selected to suppress an initiation by $\left[\mathrm{Ni}(0)\left(\mathrm{PPh}_{3}\right)_{4}\right]$, which is formed by disproportionation but requires a long induction period.

Table S 9. Radical MMA polymerizations initiated by $\mathrm{Ni}(0)$ and $\mathrm{Ni}(\mathrm{I})$ complexes with and without addition of aryl halides.

\begin{tabular}{lllllll}
\hline & complex & $\begin{array}{l}\text { equiv. } \\
\text { add }\end{array}$ & {$[\mathbf{N i}] / \mathbf{m m o l} \cdot \mathbf{L}^{-\mathbf{1}}$} & conv. / \% & chains/[Ni] & t / min \\
\hline $\mathbf{1}$ & {$\left[\mathrm{Ni}\left(\mathrm{PPh}_{3}\right)_{4}\right]$} & 0 & 0.5 & 0.8 & 0.2 & 325 \\
$\mathbf{2}$ & {$\left[\mathrm{Ni}\left(\mathrm{PPh}_{3}\right)_{4}\right]$} & $64 \mathrm{PhI}$ & 0.5 & 3.0 & 1.2 & 325 \\
$\mathbf{3}$ & {$\left[\mathrm{Ni}\left(\mathrm{PPh}_{3}\right)_{4}\right]$} & $15 \mathrm{BDB}$ & 9 & 5.1 & 0.5 & 428 \\
$\mathbf{4}$ & {$\left[(\mathrm{PPh})_{3} \mathrm{NiCl}\right]$} & 0 & 0.6 & 0.0 & 0.0 & 428 \\
$\mathbf{5}$ & {$\left[\left(\mathrm{PPh}_{3}\right)_{3} \mathrm{NiCl}\right]$} & $33 \mathrm{PhI}$ & 1.1 & 3.6 & 1.5 & 428 \\
$\mathbf{6}$ & $\mathbf{3}^{\mathrm{PPh} 3}{ }_{\mathrm{PPh} 3}$ & 0 & 16.3 & 0.7 & n.d. & 60 \\
\hline \multicolumn{2}{r}{ Reaction conditions: $70^{\circ} \mathrm{C}, 24 \mathrm{~mL}$ of MMA. a BDB is 1-bromo-3,5-dimethoxybenzene. }
\end{tabular}




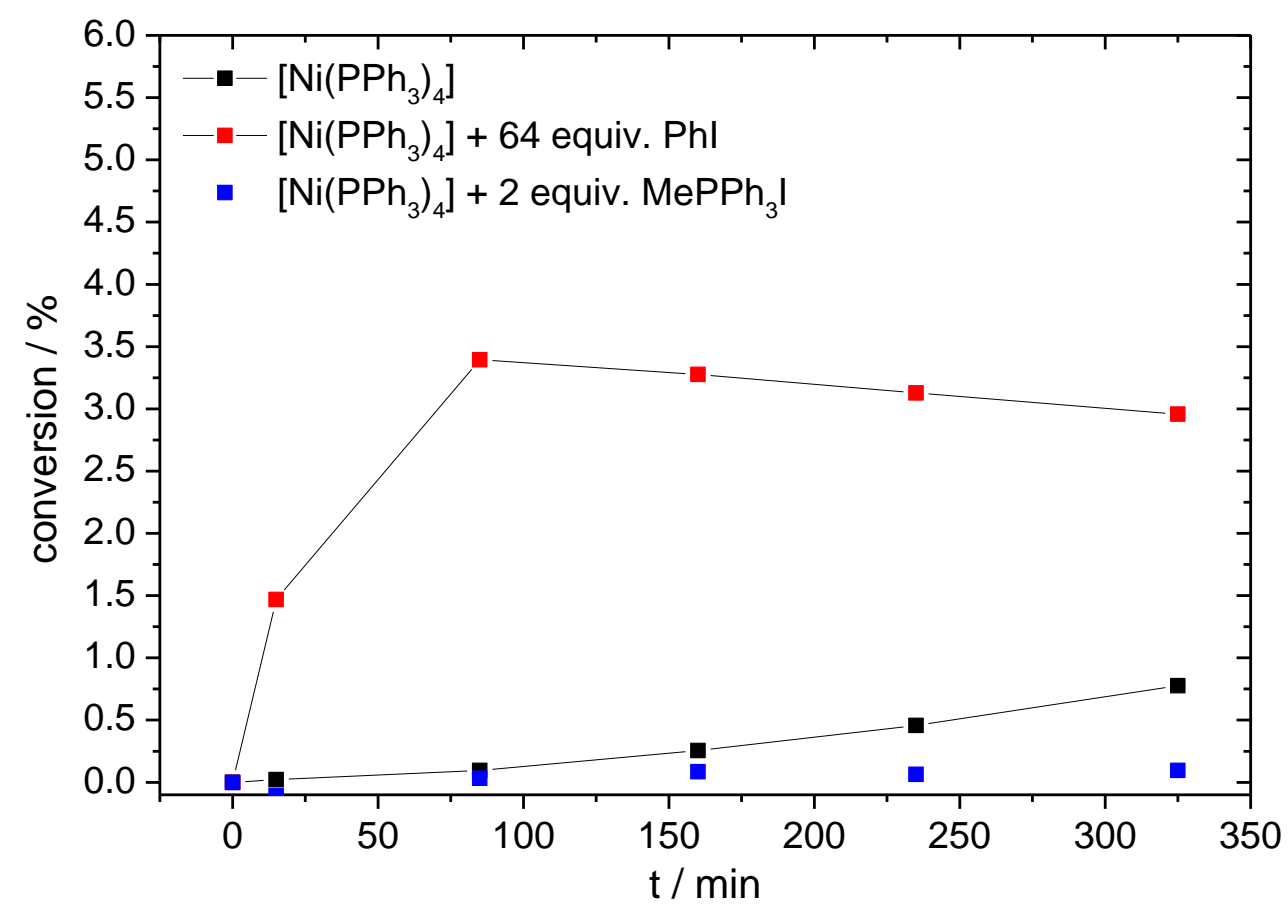

Figure S 65. Time dependent conversion for MMA homopolymerizations initiated with $\left[\mathrm{Ni}\left(\mathrm{PPh}_{3}\right)_{4}\right]$ alone (black), with 64 equiv. of $\mathrm{Ph}-\mathrm{I}$ (red) and 2 equiv. of $\mathrm{MePPh}_{3} \mathrm{I}$ (blue). The polymerization initiated with $\left[\mathrm{Ni}\left(\mathrm{PPh}_{3}\right)_{4}\right]$ shows an induction period, whereas by addition of $\mathrm{PhI}$ no induction period was observed. The addition of a phosphonium salt shows an inhibiting effect.

a)

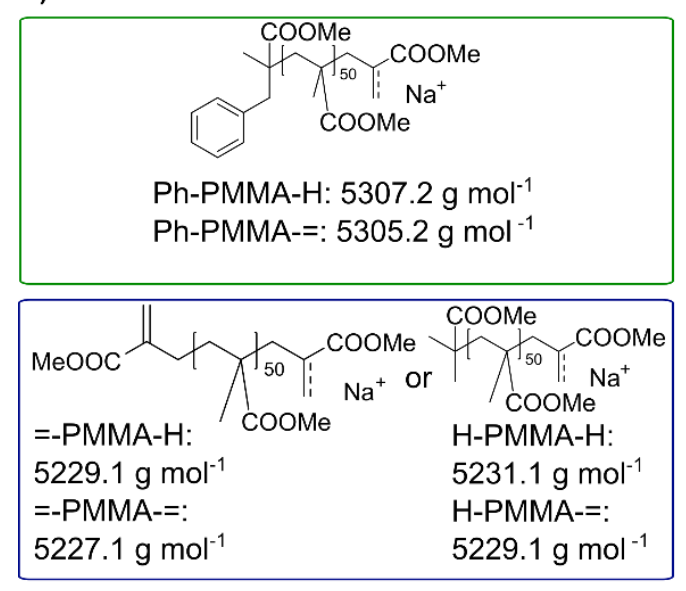

b)

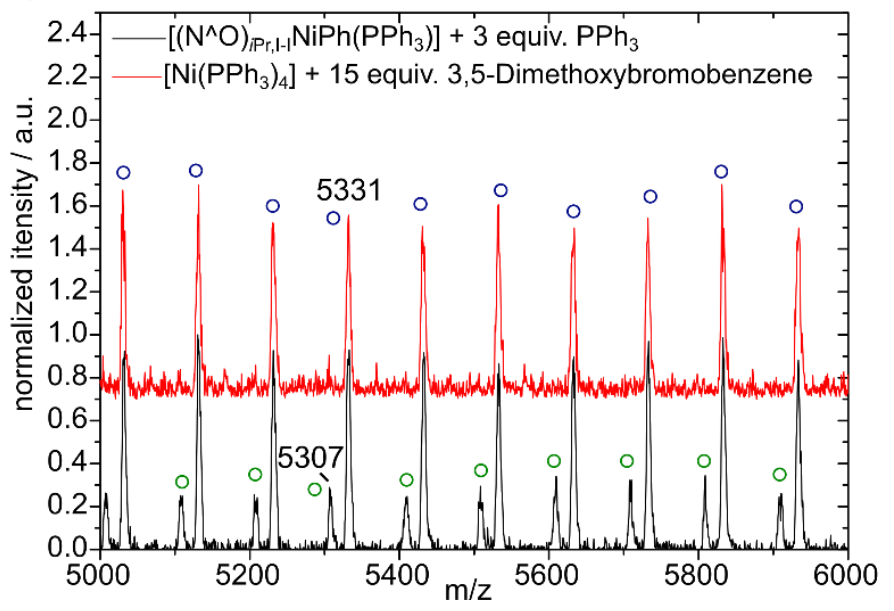

Figure S 66. MALDI-TOF MS (b) of PMMA obtained by initiation with $2^{\mathrm{Ph}}{ }_{\mathrm{PPh} 3}$ plus 3 equiv. of $\mathrm{PPh}_{3}($ black) and with $\left[\mathrm{Ni}\left(\mathrm{PPh}_{3}\right)_{4}\right]$ plus 15 equiv. 3,5-dimethoxybromobenzene (red, Table $\mathrm{S} 9$, Entry 3). The assignment of the endgroups are included (blue and green circles). The structures are shown on the left (a): PMMA with a phenyl endgroup (green) and PMMA with a double bond or a proton end-group (blue). 

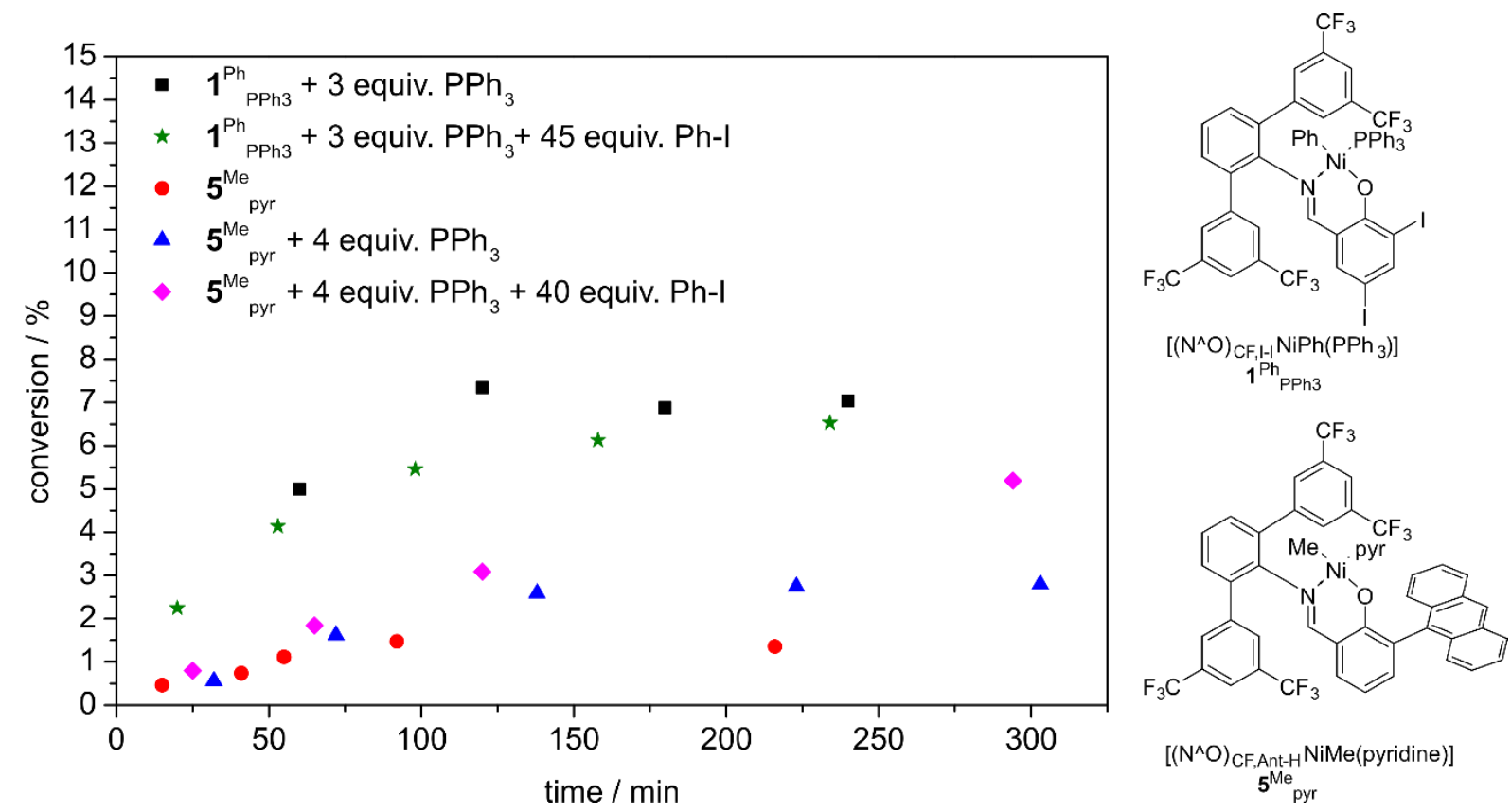

Figure S 67. MMA homopolymerizations initiated with the diiodo substituted complexes $\mathbf{1}^{\mathrm{Ph}}{ }_{\mathrm{PPh} 3}$ and 3 equiv. of $\mathrm{PPh}_{3}$ (black); $\mathbf{1}^{\mathrm{Ph}}{ }_{\mathrm{Phh}}, 3$ equiv. of $\mathrm{PPh}_{3}$ and 45 equiv. of phenyl iodide (green) in comparison with the anthryl substituted salicylaldimine complex $\left[\left(\mathrm{N}^{\wedge} \mathrm{O}\right)_{\mathrm{CF}, \mathrm{Ant}-\mathrm{H}} \mathrm{NiMe}\right.$ (pyridine) $)\left(\mathbf{5}^{\mathrm{Me}}{ }_{\mathrm{py}}\right.$, red), with addition of 4 equiv. of $\mathrm{PPh}_{3}$ (blue) and with addition of 4 equiv. of $\mathrm{PPh}_{3}$ and 40 equiv. of phenyl iodide (pink). For the iodine free ligand system $\left(5^{\mathrm{Me}}{ }_{\mathrm{pyr}}\right)$ a higher yield for a radical polymerization of MMA was observed when Ph-I was added. Reaction conditions: $70{ }^{\circ} \mathrm{C},[\mathrm{Ni}]=0.5 \mathrm{mmol} \cdot \mathrm{L}^{-1},[\mathrm{MMA}]=9.389 \mathrm{~mol} \cdot \mathrm{L}^{-1}$. 


\section{Reactivity of Ni(I) toward Organic Radicals}

a) ${ }^{1} \mathrm{H}$ NMR

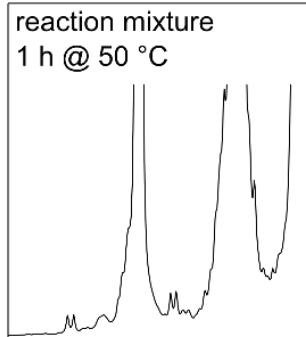

[Ni-Ph] independently synthesized

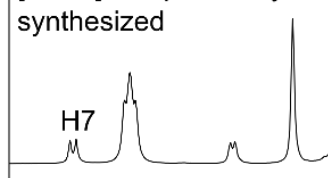

8.28 .

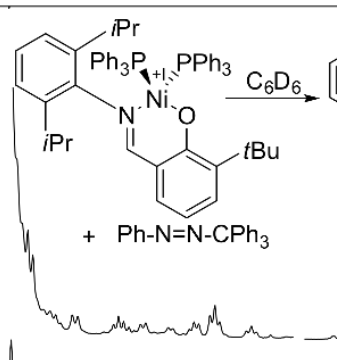

[Ni-Ph-p-H]

b) ${ }^{31} \mathrm{P}\left\{{ }^{1} \mathrm{H}\right\}$ NMR

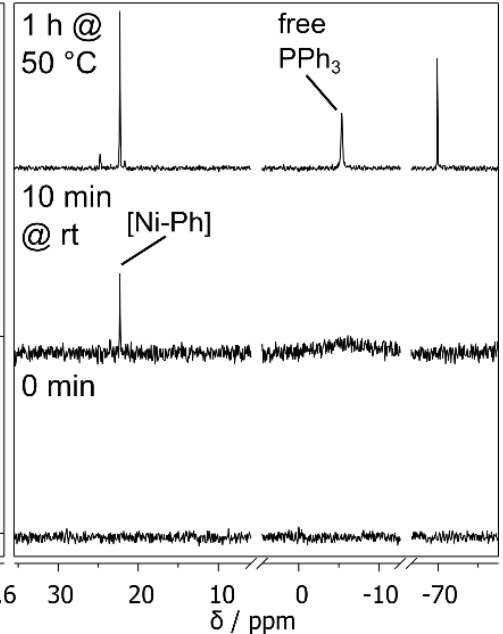

Figure $\mathbf{S}$ 68. Monitoring the reaction of $\mathbf{3}^{\mathrm{PPh} 3}{ }_{\mathrm{PPh} 3}$ with 15 equiv. of phenylazo-triphenylmethane forming the corresponding [Ni(II)-Ph] complex in $\mathrm{C}_{6} \mathrm{D}_{6}$ (inset) by ${ }^{1} \mathrm{H}$ NMR $(\mathbf{a}, 400 \mathrm{MHz}, \mathrm{RT})$ and ${ }^{31} \mathrm{P}\left\{{ }^{1} \mathrm{H}\right\} \mathrm{NMR}(\mathbf{b}, 162 \mathrm{MHz}$, $\mathrm{RT}$ ). In a the ${ }^{1} \mathrm{H}$ NMR spectrum of the independently synthesized $\mathbf{3}^{\mathrm{Ph}}{ }_{\mathrm{PPh} 3}$ and the assignment of characteristic resonances is shown at the bottom. The ${ }^{1} \mathrm{H}$ NMR spectrum of the reaction mixture of $3^{\mathrm{PPh} 3}{ }_{\mathrm{PPh} 3}$ with 15 equiv. of phenylazo-triphenylmethane after $1 \mathrm{~h}$ at $50{ }^{\circ} \mathrm{C}$ is shown at the top. b shows the reaction monitoring via ${ }^{31} \mathrm{P}\left\{{ }^{1} \mathrm{H}\right\}$ NMR spectroscopy (162 Mhz, RT). At $50{ }^{\circ} \mathrm{C}$ a unassigned phosphorus species was formed $\left(\delta\left({ }^{31} \mathrm{P}\left\{{ }^{1} \mathrm{H}\right\}=-70 \mathrm{ppm}\right)\right.$.
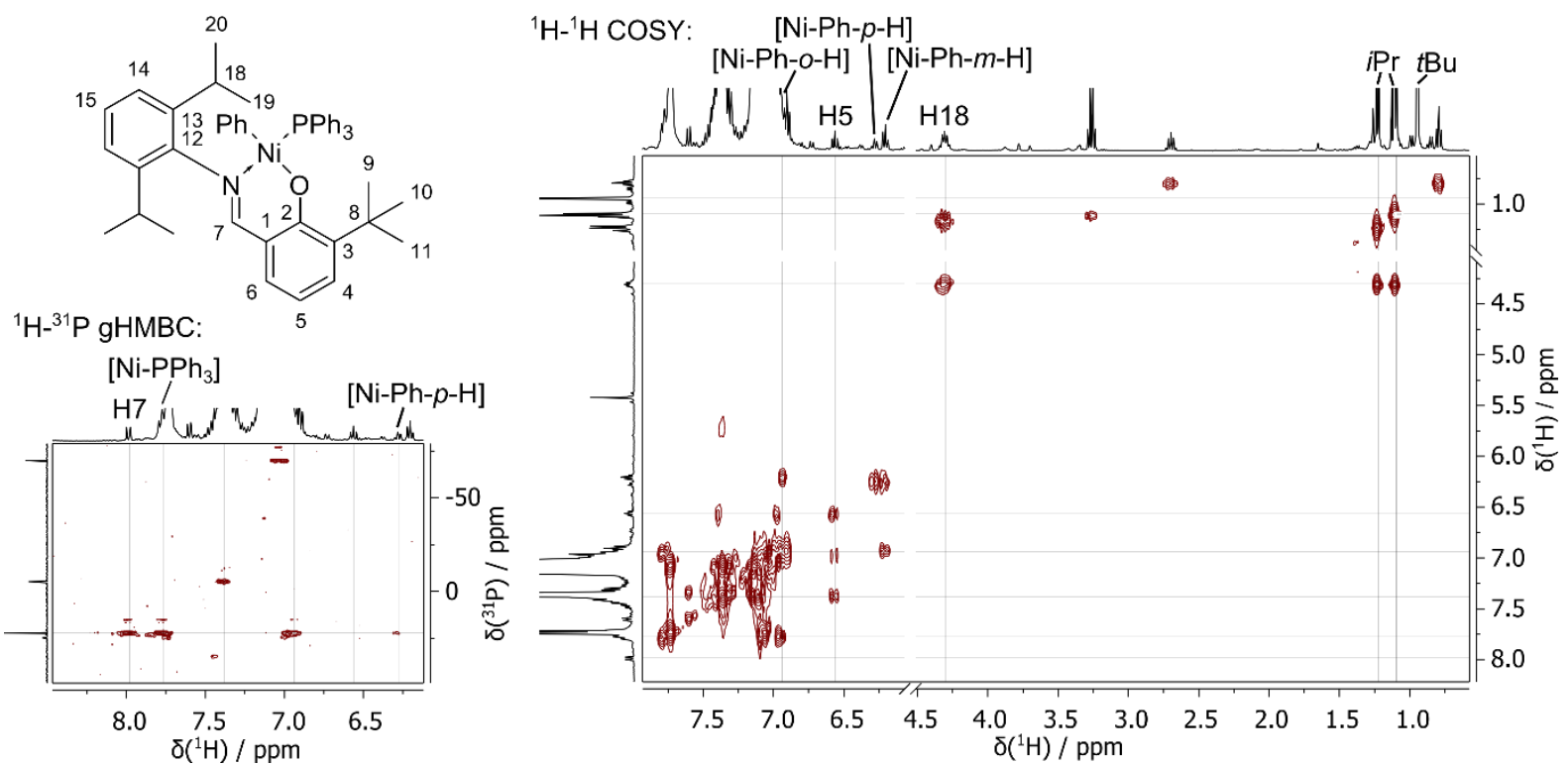

Figure S 69. ${ }^{1} \mathrm{H}_{-}{ }^{31} \mathrm{P}$ gHMBC (left bottom) and ${ }^{1} \mathrm{H}-{ }^{1} \mathrm{H}$ COSY (right, $400 \mathrm{MHz}, \mathrm{C}_{6} \mathrm{D}_{6}, \mathrm{RT}$ ) showing the mixture of $3^{\mathrm{PPh} 3}{ }_{\mathrm{PPh} 3}$ and 15 equiv. of phenylazo-triphenylmethane after $1 \mathrm{~h}$ at $50{ }^{\circ} \mathrm{C}$. Characteristic resonances are assigned to $\mathbf{3}^{\mathrm{Ph}}{ }_{\mathrm{PPh} 3}$. 


\section{Ethylene polymerization catalyst recycling studies}

\section{General procedure for pressure reactor experiments}

Polymerizations were performed in a BÜCHI steel reactor with glass-inlet. The reactor was predried under vacuum at $70^{\circ} \mathrm{C}$ for $1 \mathrm{~h} .7 .5 \mu \mathrm{mol}$ of [Ni] and the desired amount of the azo compound was weighted into separated vials in a $\mathrm{N}_{2}$ glovebox and dissolved in small amounts of toluene, respectively. Toluene and the solutions of [Ni] and the azo compound were transferred into the preheated reactor and the solution was stirred $(750 \mathrm{rpm})$. The total amount of toluene was adjusted to $100 \mathrm{~mL}$. Afterwards, the reactor was pressurized to 25 bar ethylene and the mass flow of ethylene was recorded. When no further consumption of ethylene was observed the polymerization mixtures were vented and poured into a large excess of stirred $\mathrm{MeOH}$.

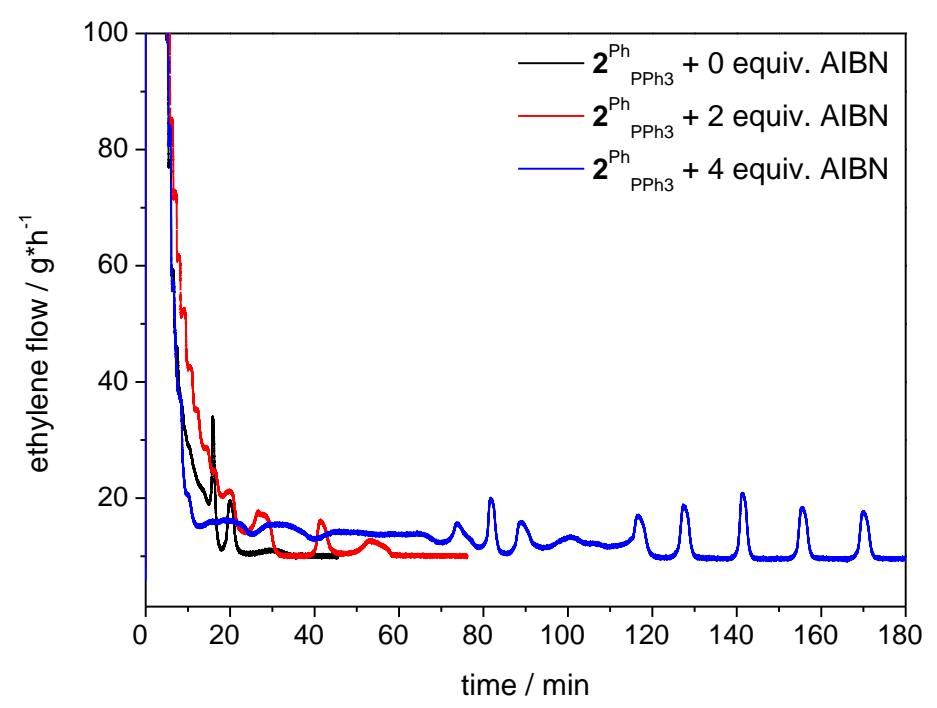

Figure S 70. Ethylene mass flow plots for the ethylene polymerizations with $2^{\mathrm{Ph}}{ }_{\mathrm{PPh} 3}$ (black), $2^{\mathrm{Ph}}{ }_{\mathrm{PPh} 3}$ with 2 equiv. of AIBN (red) and $2^{\mathrm{Ph}}{ }_{\mathrm{PPh} 3}$ with 4 equiv. of AIBN (blue) at 25 bar ethylene pressure at $25{ }^{\circ} \mathrm{C}$ in $100 \mathrm{~mL}$ of toluene. Polymerizations were quenched when no further ethylene consumption was observed. A prolonged activity with increasing AIBN amount was observed (black vs. red vs. blue). 
Table S 10. Ethylene polymerizations with catalyst precursor $2^{\mathrm{Ph}} \mathrm{PPh} 3$ with or without addition of azo compounds at $25^{\circ} \mathrm{C}$.

\begin{tabular}{|c|c|c|c|c|c|c|c|c|c|c|c|}
\hline $\begin{array}{l}\text { Ent } \\
\text { ry }\end{array}$ & $\begin{array}{l}\text { Compl } \\
\text { ex }\end{array}$ & $\begin{array}{l}\text { equiv. } \\
\text { Add }\end{array}$ & $\begin{array}{l}\text { Yiel } \\
\text { d / } \\
\text { g }\end{array}$ & $\begin{array}{l}\text { Lifetime } \\
\text { a / min }\end{array}$ & TON $^{b}$ & TOF $^{c}$ & $\begin{array}{l}M_{\mathbf{n}}{ }^{\mathrm{d}} \quad / \\
10^{3} \\
\mathbf{g} \cdot \mathbf{m o l}^{-1}\end{array}$ & $\begin{array}{r}M_{\mathrm{w}} \\
/ \boldsymbol{M}_{\mathrm{n}}{ }^{d}\end{array}$ & $\begin{array}{l}\text { PE } \\
\text { chains/ } \\
{[\mathrm{Ni}]^{\mathrm{e}}}\end{array}$ & $\begin{array}{l}T_{\mathrm{m}} \\
\mathrm{f} / \\
{ }^{\circ} \mathrm{C} \\
\end{array}$ & $\begin{array}{l}\text { Cryst } \\
\text { f / \% }\end{array}$ \\
\hline $\mathbf{1}$ & $\mathbf{2}^{\mathrm{Ph} \mathrm{PPh}_{3}}$ & o & 0.92 & 30 & 4370 & 8750 & 156 & 1.6 & 0.8 & 132 & 55 \\
\hline 2 & $2^{\mathrm{Ph}_{\mathrm{PPh}_{3}}}$ & 2 PAT & 1.87 & 50 & 8890 & 10670 & 154 & 1.8 & 1.6 & 132 & 57 \\
\hline 3 & $2^{\mathrm{Ph}_{\mathrm{PPh}}}$ & ${ }_{25}$ PAT & 0.02 & 20 & 100 & 290 & 3 & $4 \cdot 5$ & 0.4 & 125 & 70 \\
\hline 4 & $2^{\mathrm{Ph} \mathrm{PPh}_{3}}$ & 2 AIBN & 2.28 & $6 o$ & 10840 & 10840 & 278 & 1.3 & 1.1 & 132 & 51 \\
\hline 5 & $2^{\mathrm{Ph}_{\mathrm{PPh}_{3}}}$ & 4 AIBN & $5 \cdot 77$ & $>180$ & 27430 & 9140 & 285 & 1.3 & 2.7 & 132 & 47 \\
\hline 6 & $2^{\mathrm{Ph}_{\mathrm{PPh}}}$ & 6 AIBN & 6.57 & $>190$ & 31230 & 9860 & 298 & 1.3 & 2.9 & 132 & 49 \\
\hline 7 & $2^{\mathrm{Ph}_{\mathrm{PPh}_{3}}}$ & 8 AIBN & 0.63 & 30 & 3000 & 5990 & 186 & 1.7 & 0.5 & 132 & 54 \\
\hline 8 & $\mathbf{2}^{\mathrm{Ph}_{\mathrm{PPh}}}$ & 25 AIBN & 0.01 & 25 & 48 & 114 & 4 & 4.5 & 0.4 & 125 & 70 \\
\hline
\end{tabular}

Reaction conditions: $100 \mathrm{~mL}$ of toluene, $7.5 \mu \mathrm{mol}$ of [Ni], 25 bar of E, $25^{\circ} \mathrm{C}$. (a) Lifetime is determined when no further consumption of $E$ is detected by mass flow meter. (b) $n(E) \cdot n([N i])^{-1}$. (c) $n(E) \cdot n([N i])^{-1} \cdot h^{-1}$. (d) Determined by SEC in 1,2,4-trichlorobenzene at $160{ }^{\circ} \mathrm{C}$ vs. linear PE standards. (e) $\mathrm{m}(\mathrm{PE}) \cdot \mathrm{M}(\mathrm{PE})^{-1} \cdot \mathrm{n}(\mathrm{Ni})^{-1}$. (f) Determined by DSC from the second heating curve. Crystallinity was calculated by using a heat of fusion of $293 \mathrm{~J} \mathrm{~g}^{-1}$ for $100 \%$ crystallinity of linear PE. ${ }^{5}$

Note that an inherent prerequisite for this scenario is a sufficient stability of the intermediate $[\mathrm{Ni}(\mathrm{I})]$ species, which was shown to be strongly dependent on the $\mathrm{N}^{\wedge} \mathrm{O}$ ligand structure $\left(3^{\mathrm{PPh} 3} \mathrm{PPh} 3\right.$ Vs. $4^{\mathrm{PPh} 3} \mathrm{PPh} 3$, vide supra). As a proof of principle, we carried out polymerizations of ethylene in the presence of azo compounds with complex $2^{\mathrm{Ph}}{ }_{\mathrm{PPh} 3}$ (Table S 10). We were using $\mathbf{2}^{\mathrm{Ph}}{ }_{\mathrm{PPh} 3}$ as catalyst precursor because a $[\mathrm{Ni}(\mathrm{I})]$ complex was observed when a sample of $\mathbf{2}^{\mathrm{Ph}}{ }_{\mathrm{PPh} 3}$ was heated and the reaction was monitored by EPR spectroscopy. ${ }^{42}$ This indicates that the corresponding $[\mathrm{Ni}(\mathrm{I})]$ is sufficiently stable for reactivation attempts. In addition, complex $3^{\mathrm{Ph}}{ }_{\mathrm{PPh} 3}$ was described to be not polymerization active without a phosphine scavenger. ${ }^{43}$

Polymerizations were conducted at $25^{\circ} \mathrm{C}, 25$ bar of $\mathrm{E}$ in $100 \mathrm{~mL}$ of toluene. Both, phenylazotriphenylmethane (PAT) (Table S 10, Entry 2) and AIBN (Table S 10, Entries 4-6) were able to enhance the yield of the polymerizations in comparison to a polymerization without any addition (Table S 10, Entry 1). The best result was obtained by addition of 6 equiv. of AIBN, resulting in a 7 times higher yield of PE (Table S 10, Entry 1 vs. 6). Interestingly, large amounts of azo compound completely deactivated the polymerization (Table S 10, Entries 3,7,8).

The increase of the yield is reasoned by a prolonged lifetime of the catalyst in the presence of azo compounds (Figure S 70). Without addition of AIBN the polymerization was active for ca. 30 min, while with addition of 6 equiv. of AIBN ethylene consumption for more than 190 minutes was observed (Table S 10, Entry 1 vs. 6). In the absence or presence of PAT (2 equiv.) and with additional (2,4 and 6 equiv.) AIBN (Table S 10, Entries 1-2 and 4-6) the turnover frequencies (TOF) remained almost constant $\left(9.000-11.000 \mathrm{~h}^{-1}\right)$. This indicates, that in all cases the actual active species of chain growth were identical. Further support was given by DSC analysis of the polymers formed, showing that the melting points, and therefore the microstructure of the PE formed remained unchanged (Table S 10, Entries 1-2 and 4-6). SEC $\left(160{ }^{\circ} \mathrm{C}\right.$, SEC in 1,2,4-trichlorobenzene at $160^{\circ} \mathrm{C}$ vs. linear PE standards) showed that the 
molecular weight distribution got smaller (Table S 10, Entry 1 vs. 4-6) and that $M_{\mathrm{n}}$ of PE formed increased with the enhanced lifetime of the catalyst. These results prove the promising principle of reactivation by scavenging intermediate $[\mathrm{Ni}(\mathrm{I})]$ species by sources of organic radicals and transformation to a catalytically active species.

However, it seems that this reaction is sensitive to differently substituted $\mathrm{N}^{\wedge} \mathrm{O}$ ligands and polymerization conditions. E.g. attempted reactivation experiments at $50{ }^{\circ} \mathrm{C}$ did not show a large beneficial effect up to 100 equiv. AIBN. The best result was a 1.7 times higher yield with 50 equiv. AIBN in comparison to the polymerization in the absence of AIBN. 


\section{References}

(1) Stoll, S.; Schweiger, A. J. Magn. Reson. 2006, 178, 42-55.

(2) X-Red version 1.31 ed. 2005, p Stoe Data Reduction Programm, Darmstadt, Germany.

(3) Sheldrick, G. M. Acta Crystallogr. Sect. A 2008, 64, 112-22.

(4) Farrugia, L. J. Appl. Crystallogr. 1997, 30, 565.

(5) Wunderlich, B.; Czornyj, G. Macromolecules 1977, 10, 906-13.

(6) Göttker-Schnetmann, I.; Wehrmann, P.; Röhr, C.; Mecking, S. Organometallics 2007, 26, 2348-62.

(7) Wang, C. M.; Friedrich, S.; Younkin, T. R.; Li, R. T.; Grubbs, R. H.; Bansleben, D. A.; Day, M. W. Organometallics 1998, 17, 3149-51.

(8) Cohen, S. G.; Wang, C. H. J. Am. Chem. Soc. 1953, 75, 5504-7.

(9) Zeller, A.; Herdtweck, E.; Strassner, T. Eur. J. Inorg. Chem. 2003, 1802-6.

(10) Berkefeld, A.; Mecking, S. J. Am. Chem. Soc. 2009, 131, 1565-74.

(11) Soshnikov, I. E.; Semikolenova, N. V.; Zakharov, V. A.; Möller, H. M.; Ölscher, F.; Osichow, A.; Göttker-Schnettmann, I.; Mecking, S.; Talsi, E. P.; Bryliakov, K. P. Chem. Eur. J. 2013, 19, 11409-17.

(12) Bauers, F. M.; Mecking, S. Macromolecules 2001, 34, 1165-71.

(13) (a) Velian, A.; Lin, S.; Miller, A. J. M.; Day, M. W.; Agapie, T. J. Am. Chem. Soc. 2010, 132, 6296-7; (b) Gruger, N.; Wadepohl, H.; Gade, L. H. Dalton Trans. 2012, 41, 14028-30; (c) Laskowski, C. A.; Bungum, D. J.; Baldwin, S. M.; Del Ciello, S. A.; Iluc, V. M.; Hillhouse, G. L. J. Am. Chem. Soc. 2013, 135, 18272-5; (d) Laskowski, C. A.; Morello, G. R.; Saouma, C. T.; Cundari, T. R.; Hillhouse, G. L. Chem. Sci. 2013, 4, 170-4; (e) Lipschutz, M. I.; Yang, X.; Chatterjee, R.; Tilley, T. D. J. Am. Chem. Soc. 2013, 135, 15298-301; (f) Beck, R.; Johnson, S. A. Organometallics 2013; (g) Page, M. J.; Lu, W. Y.; Poulten, R. C.; Carter, E.; Algarra, A. G.; Kariuki, B. M.; Macgregor, S. A.; Mahon, M. F.; Cavell, K. J.; Murphy, D. M.; Whittlesey, M. K. Chem. Eur. J. 2013, 19, 2158-67; (h) Wu, J.; Nova, A.; Balcells, D.; Brudvig, G. W.; Dai, W.; Guard, M. L. M.; Hazari, N.; Lin, P.-H.; Pokhrel, R.; Takase, M. K. Chem. Eur. J. 2014, 20, 5327-37; (i) Reed, B. R.; Stoian, S. A.; Lord, R. L.; Groysman, S. Chem. Commun. 2015, 51, 6496-9; (j) Kreye, M.; Freytag, M.; Jones, P. G.; Williard, P. G.; Bernskoetter, W. H.; Walter, M. D. Chem. Commun. 2015.

(14) (a) Review: Rosen, B. M.; Quasdorf, K. W.; Wilson, D. A.; Zhang, N.; Resmerita, A.-M.; Garg, N. K.; Percec, V. Chem. Rev. 2011, 111, 1346-416; (b) Li, Z.; Jiang, Y.-Y.; Fu, Y. Chem. Eur. J. 2012, 18, 4345-57; (c) Yan, C.-S.; Peng, Y.; Xu, X.-B.; Wang, Y.-W. Chem. Eur. J. 2012, 18, 6039-48; (d) Zultanski, S. L.; Fu, G. C. J. Am. Chem. Soc. 2013, 135, 624-7.

(15) Spek, A. L. Acta Crystallogr., Sect D 2009, 65, 148-55.

(16) Heimbach, P. Angew. Chem. 1964, 76, 586-.

(17) Kuchen, W.; Peters, W.; Sünkeler, M.; Wiskemann, R.; Wunderlich, H. Z. Anorg. Allg. Chem. 1998, 624, 1956-64.

(18) (a) Orpen, A. G.; Connelly, N. G. Organometallics 1990, 9, 1206-10; (b) Fey, N.; Orpen, A. G.; Harvey, J. N. Coord. Chem. Rev. 2009, 253, 704-22.

(19) Wang, H. Y.; Meng, X.; Jin, G. X. Dalton Trans. 2006, 2579-85.

(20) (a) Azevedo, F.; Freire, C.; de Castro, B. Polyhedron 2002, 21, 1695-705; (b) Bai, G.; Wei, P.; Stephan, D. W. Organometallics 2005, 24, 5901-8; (c) Marlier, E. E.; Tereniak, S. J.; Ding, K.; Mulliken, J. E.; Lu, C. C. Inorg. Chem. 2011, 50, 9290-9; (d) Chambers, G. M.; Mitra, J.; Rauchfuss, T. B.; Stein, M. Inorg. Chem. 2014, 53, 4243-9.

(21) Göttker-Schnettmann, I.; Mecking, S. Unpublished results.

(22) (a) Kim, I.; Kwak, C. H.; Kim, J. S.; Ha, C.-S. Appl. Catal., A. 2005, 287, 98-107; (b) Connor, E. F.; Younkin, T. R.; Henderson, J. I.; Waltman, A. W.; Grubbs, R. H. Chem. Commun. 2003, 2272-3. 
(23) Ellis, D. D.; Spek, A. L. Acta Crystallogr. Sect. C 2000, 56, 1067-70.

(24) Pickel, M.; Casper, T.; Rahm, A.; Dambouwy, C.; Chen, P. Helv. Chim. Acta 2002, 85, 4337-52.

(25) Duval-Terrié, C.; Lebrun, L. J. Chem. Educ. 2006, 83, 443.

(26) (a) Leblanc, A.; Broyer, J.-P.; Boisson, C.; Spitz, R.; Monteil, V. Pure Appl. Chem. 2012, 84, 2113-20; (b) Leblanc, A.; Grau, E.; Broyer, J.-P.; Boisson, C.; Spitz, R.; Monteil, V. Macromolecules 2011, 44, 3293-301.

(27) (a) Bevington, J. C.; Melville, H. W.; Taylor, R. P. J. Polym. Sci. 1954, 12, 449-59; (b) Bevington, J. C.; Melville, H. W.; Taylor, R. P. J. Polym. Sci. 1954, 14, 463-76.

(28) Grau, E.; Broyer, J.-P.; Boisson, C.; Spitz, R.; Monteil, V. Polym. Chem. 2011, 2, 2328 33.

(29) (a) Moad, G.; Solomon, D.; Spurling, T.; Johns, S.; Willing, R. Aust. J. Chem. 1986, 39, 43-50; (b) Brar, A. S.; Singh, G.; Shankar, R. J. Mol. Struct. 2004, 703, 69-81.

(30) (a) Randall, J. C. J. Macromol. Sci., Polym. Rev. 1989, 29, 201-317; (b) Cotts, P. M.; Guan, Z.; McCord, E.; McLain, S. Macromolecules 2000, 33, 6945-52; (c) Wiedemann, T.; Voit, G.; Tchernook, A.; Roesle, P.; Göttker-Schnetmann, I.; Mecking, S. J. Am. Chem. Soc. 2014, 136, 2078-85.

(31) Matos, I.; Ascenso, J.; Lemos, M.; Fan, Z.; Yuan, J.; Farinha, J.; Lemos, F.; Gonçalves da Silva, A. P. S.; Marques, M. J. Polym. Res. 2013, 20, 1-16.

(32) (a) Jenkins, J. C.; Brookhart, M. J. Am. Chem. Soc. 2004, 126, 5827-42; (b) Waltman, A. W.; Younkin, T. R.; Grubbs, R. H. Organometallics 2004, 23, 5121-3.

(33) (a) Langer, J.; Fischer, R.; Görls, H.; Theyssen, N.; Walther, D. Z. Anorg. Allg. Chem. 2007, 633, 557-62; (b) Beck, R.; Shoshani, M.; Krasinkiewicz, J.; Hatnean, J. A.; Johnson, S. A. Dalton Trans. 2013, 42, 1461-75.

(34) Lejkowski, M. L.; Lindner, R.; Kageyama, T.; Bódizs, G. É.; Plessow, P. N.; Müller, I. B.; Schäfer, A.; Rominger, F.; Hofmann, P.; Futter, C.; Schunk, S. A.; Limbach, M. Chem. Eur. J. 2012, 18, 14017-25.

(35) Berkefeld, A.; Drexler, M.; Möller, H. M.; Mecking, S. J. Am. Chem. Soc. 2009, 131, 12613-22.

(36) Rünzi, T.; Guironnet, D.; Göttker-Schnetmann, I.; Mecking, S. J. Am. Chem. Soc. 2010, 132, 16623-30.

(37) Liu, Y.; Mao, D.; Xu, D.; Xu, Z.; Zhang, Y. Synth. Commun. 2007, 37, 4389-97.

(38) (a) Horner, L.; Hoffmann, H. Chem. Ber. 1958, 91, 50-2; (b) Horner, L.; Hoffmann, H. Chem. Ber. 1958, 91, 45-9.

(39) (a) Rünzi, T.; Tritschler, U.; Roesle, P.; Göttker-Schnetmann, I.; Möller, H. M.; Caporaso, L.; Poater, A.; Cavallo, L.; Mecking, S. Organometallics 2012, 31, 8388-406; (b) Segelstein , B. E.; Butler, T. W.; Chenard, B. L. J. Org. Chem. 1995, 60, 12-3.

(40) Kotake, Y.; Janzen, E. G. J. Am. Chem. Soc. 1988, 110, 3699-701.

(41) Maillard, P.; Massot, J. C.; Giannotti, C. J. Organomet. Chem. 1978, 159, 219-27.

(42) Monteil, V. Actual. Chimique 2012, 30-6.

(43) Younkin, T. R.; Connor, E. F.; Henderson, J. I.; Friedrich, S. K.; Grubbs, R. H.; Bansleben, D. A. Science 2000, 287, 460-2. 